\title{
Platelet responsiveness and function during storage : implications for platelet transfusion therapy
}

Citation for published version (APA):

Cauwenberghs, S. (2007). Platelet responsiveness and function during storage : implications for platelet transfusion therapy. [Doctoral Thesis, Maastricht University]. Maastricht University. https://doi.org/10.26481/dis.20070620sc

Document status and date:

Published: 01/01/2007

DOI:

$10.26481 /$ dis.20070620sc

Document Version:

Publisher's PDF, also known as Version of record

\section{Please check the document version of this publication:}

- A submitted manuscript is the version of the article upon submission and before peer-review. There can be important differences between the submitted version and the official published version of record.

People interested in the research are advised to contact the author for the final version of the publication, or visit the DOI to the publisher's website.

- The final author version and the galley proof are versions of the publication after peer review.

- The final published version features the final layout of the paper including the volume, issue and page numbers.

Link to publication

\footnotetext{
General rights rights.

- You may freely distribute the URL identifying the publication in the public portal. please follow below link for the End User Agreement:

www.umlib.nl/taverne-license

Take down policy

If you believe that this document breaches copyright please contact us at:

repository@maastrichtuniversity.nl

providing details and we will investigate your claim.
}

Copyright and moral rights for the publications made accessible in the public portal are retained by the authors and/or other copyright owners and it is a condition of accessing publications that users recognise and abide by the legal requirements associated with these

- Users may download and print one copy of any publication from the public portal for the purpose of private study or research.

- You may not further distribute the material or use it for any profit-making activity or commercial gain

If the publication is distributed under the terms of Article $25 \mathrm{fa}$ of the Dutch Copyright Act, indicated by the "Taverne" license above, 


\title{
Platelet responsiveness and function during storage
}

\author{
implications for \\ platelet transfusion therapy
}

Sandra Cauwenberghs 
Platelet responsiveness and function during storage

Implications for platelet transfusion therapy

Thesis Cardiovascular Research Institute Maastricht (CARIM)

and Blood Bank South East, the Netherlands -

with summary in Dutch

ISBN/EAN: 978-90-9021968-4

(c) S. Cauwenberghs, Vilvoorde, Belgium 2007

Printed by: Egberghs NV

Cover design by: Frank le Pair 


\title{
Platelet responsiveness and function during storage
}

\author{
implications for \\ platelet transfusion therapy
}

PROEFSCHRIFT

ter verkrijging van de graad van doctor

aan de Universiteit Maastricht

op gezag van de Rector Magnificus,

Prof. mr. G.P.M.F. Mols

volgens het besluit van het College van Decanen,

in het openbaar te verdedigen op

woensdag 20 juni 2007 om 12.00 uur

door

Sandra Cauwenberghs

Geboren op 16 april 1979 te Anderlecht, België 


\section{Promotor}

Prof. Dr. J. Rosing

\section{Copromotores}

Dr. J.W.M. Heemskerk

Dr. J. Curvers

\section{Beoordelingscommissie}

Prof. Dr. F.C.S. Ramaekers, voorzitter

Prof. Dr. Ph.G. de Groot (Universiteit Utrecht)

Prof. Dr. A. Brand (Sanquin Bloedbank - Universiteit Leiden)

Prof. Dr. H. ten Cate

Dr. M.G.A. oude Egbrink

This thesis was supported by the Landsteiner Foundation for Blood Transfusion Research, Amsterdam (0219).

Financial support by the Netherlands Heart Foundation and by the Dr. Ir. van de Laar Foundation for the publication of this thesis is gratefully aknowledged.

Additional financial support for the publication of this thesis by Sanquin Blood Supply Foundation, by Fresenius HemoCare Netherlands BV and by Baxter Fenwal Inc is gratefully acknowledged. 


\section{Contents}

$\begin{array}{lll}\text { Chapter } 1 & \text { General introduction } & 7\end{array}$

Chapter $2 \quad$ Hemostatic and signaling functions of transfused platelets 19

Transfusion Medicine Reviews. 2007 (in press)

Chapter 3 Plasma ecto-nucleotidases prevent desensitization of

purinergic receptors in stored platelets: importance for platelet activity during thrombus formation

Transfusion. 2006; 46:1018-28

Chapter $4 \quad$ Platelet ADP response deteriorates in synthetic storage media

Transfusion. 2006; 46:204-12

Chapter 5 Shedding of procoagulant microparticles from unstimulated platelets by integrin-mediated destabilization of actin cytoskeleton

FEBS Letters. 2006; 580:5313-20

Chapter 6 Effect of storage on agonist-induced calcium responses in platelets: a pilot study

(to be submitted)

Chapter $7 \quad$ Novel methodology for assessment of prophylactic platelet

transfusion therapy by measuring increased thrombus

formation and thrombin generation

British Journal of Haemathology. 2007; 136:480-90

Chapter 8 General discussion

Samenvatting

References

Dankwoord

Curriculum vitae

Publications 

Chapter 1

\section{General introduction}


A primary function of blood platelets is to maintain the normal circulation of blood by preserving vascular integrity and to ensure hemostasis. Circulating platelets retain their non-activated state as long as they are continuously exposed to vessel wall-derived platelet inhibitors, such as prostacyclin, nitric oxide and ecto-nucleotidases. ${ }^{1}$ Upon vessel wall injury, the platelets become activated by contact with subendothelial structures, such as collagen and von Willebrand factor (VWF). They firmly adhere to the site of lesion, form thrombi and initiate coagulation to control hemorrhage after injury. Platelets are produced from megakaryocytes in the bone marrow at so-called pro-platelet ends. At these sites, membrane receptor proteins, internal granules and organelles assemble via microtubuli to form the pro-platelet cell body. ${ }^{2}$ Once released, mature platelets circulate in the blood at concentrations of 150-400 $x$ $10^{9} / \mathrm{L}$ and with a mean half-life of 4.4 days (range of 2.9 to 5.9 days). ${ }^{3}$ In some disease states, the number of circulating platelets is low due to a reduced production or an increased clearance by the spleen. These patients may require prophylactic or therapeutic platelet transfusion to reduce the risk of bleeding after vascular damage.

\section{Aspects of platelet signaling}

The signal transduction processes mediating platelet activation have been extensively described by others. ${ }^{4-6}$ Here, only those pathways relevant for the present thesis are briefly introduced. Under high-shear conditions, platelet adhesion to von Willebrand factor - a large protein secreted by endothelial cells and bound to collagen -, is one of the first steps in thrombus formation. ${ }^{7}$ By interacting with von Willebrand factor, via the glycoprotein (GP)Ib-V-IX complex, platelets are slowed down and start to roll over the von Willebrand factorcollagen surface (figure 1). ${ }^{8}$ Subsequent stable adhesion takes place via the collagen receptors, GPVI and integrin $\alpha 2 \beta 1$ (GPla-lla). ${ }^{9}$ Platelet-collagen interaction via GPVI immediately results in activation of the cells. ${ }^{10}$ They change shape, express activated integrins, procoagulant phospholipids and release the paracrine agonists ATP, ADP and thromboxane $\mathrm{A}_{2} \cdot{ }^{11}$ The secreted compounds 


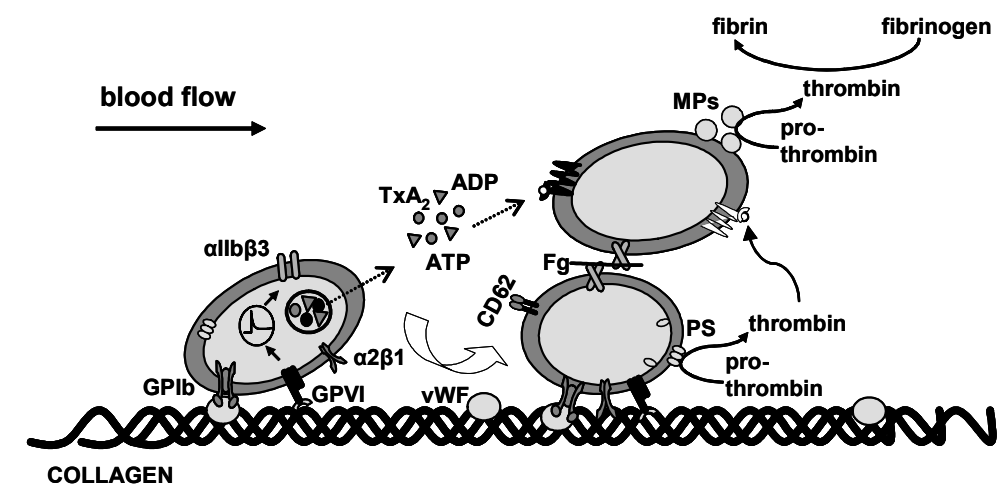

Figure 1. Principle of platelet adhesion and activation during thrombus and clot formation. When vascular damage occurs, platelets come into contact with subendothelial components, like collagen and von Willebrand factor (VWF), which is bound to the exposed collagen. Interaction between the adhesion receptor GPIb and immobilized vWF slows down platelets, which start to roll over the collagen surface. This translocation is followed by firm adhesion to collagen via the collagen receptors, GPVI and integrin a2 $\beta 1$ (GPla-lla). Platelet interaction with collagen gives rise to mobilization of intracellular $\mathrm{Ca}^{2+}$, which in turn causes release of secondary mediators [thromboxane $\mathrm{A}_{2}\left(\mathrm{TxA}_{2}\right), \mathrm{ADP}$ and ATP], leads to surface exposure of P-selectin (CD62) and PS, and to activation of the integrin allb $\beta 3$ (GPIlb-Illa). Fibrinogen (Fg) binds to the activated allb $\beta 3$, allowing platelets to attach to each other, forming aggregates. This platelet-platelet plug formation can be accompanied by a platelet-dependent procoagulant response. Coagulation factors bind to PS exposed at the activated platelet surface, where prothrombin is converted into thrombin. Thrombin by itself further activates platelets and converts fibrinogen into fibrin, making a firm platelet clot sealing the wound. The generation of platelet procoagulant activity is accompanied by the shedding of microparticles (MP), which in addition enhance coagulation.

together with thrombin, which is locally generated, activate and recruit neighboring platelets. A stable multi-platelet aggregate is formed by plateletplatelet interactions via activated integrin allbß3 (GPIIb-IIla) and fibrinogen. ${ }^{12,13}$ Thrombin generated at the site of a thrombus converts plasma fibrinogen into fibrin, which leads to the formation of a stable platelet thrombus. In addition, procoagulant microparticles are shed from the surface of activated platelets, which further support the thrombin generation. ${ }^{14}$

The platelet secretion products, ATP and ADP, appear to be of major importance for the progression of platelet activation. ${ }^{11,15,16}$ Figure 2 gives an overview of the signaling routes activated by these purinergic compounds. Secreted ATP binds to the ionotropic $P 2 X_{1}$ receptor, a cation channel that allows influx of $\mathrm{Ca}^{2+}$ into platelets. Elevation of cytosolic $\mathrm{Ca}^{2+}$ concentration via $\mathrm{P} 2 \mathrm{X}_{1}$ provokes reorganization of the actin cytoskeleton by activation of myosin light-chain kinase, which results in platelet shape change. ${ }^{17}$ Apart from this, 
$\mathrm{P} 2 \mathrm{X}_{1}$ receptor signaling, by an unknown pathway, stimulates the protein kinase, extracellular signal-regulated kinase 2 (Erk2), which amplifies dense granule release and platelet aggregation. ${ }^{18}$ It is believed that the role of the $\mathrm{P}_{2} \mathrm{X}_{1}$ receptor also extends to amplification of other signaling pathways, e.g. those evoked by thromboxane $A_{2}$ and thrombin receptor stimulation. ${ }^{19}$

Like the PAR thrombin receptors, the ADP receptor $P 2 Y_{1}$ couples to the $G_{q}$ protein, which activates phospholipase C- $\beta 2$ (PLC $\beta 2)$. This effector enzyme converts phosphatidylinositol 4,5-bisphosphate $\left(\mathrm{PIP}_{2}\right)$ into inositol 1,4,5triphosphate (IP3) and diacylglycerol. ${ }^{20}$ Binding of IP3 to its receptor in the membrane of intracellular $\mathrm{Ca}^{2+}$ stores results in mobilization of $\mathrm{Ca}^{2+}$ ions from these stores. ${ }^{21}$ Mobilized $\mathrm{Ca}^{2+}$ activates several $\mathrm{Ca}^{2+}$-dependent kinases, like myosin light-chain kinase and $\mathrm{Ca}^{2+}$-calmodulin kinase, which mediate the shape

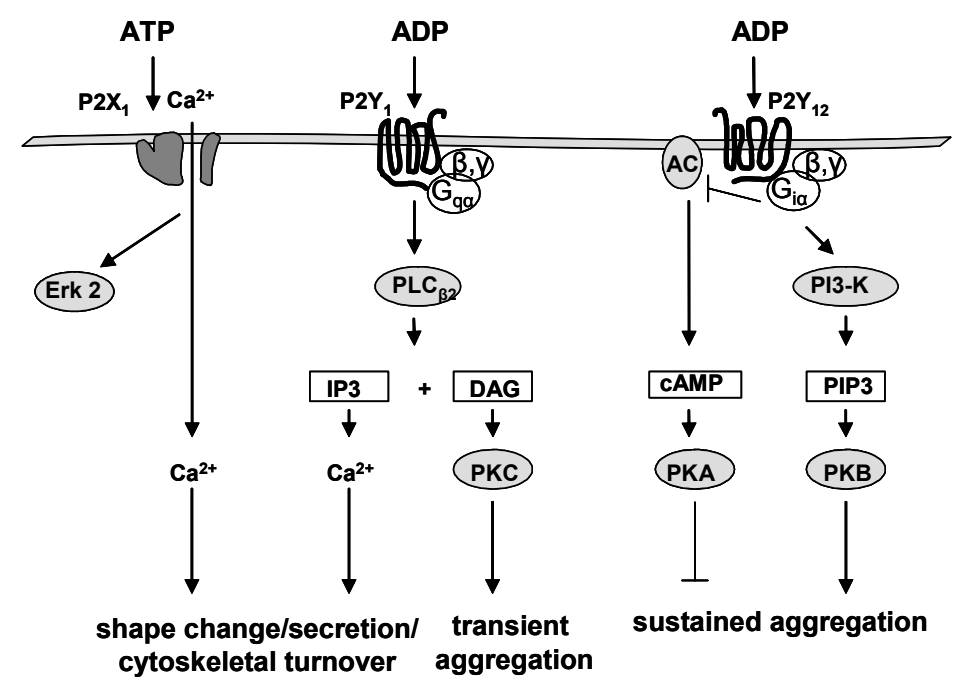

Figure 2. Platelet purinergic receptor signaling. Platelet activation by agonists like collagen results in secretion of ATP and ADP from the dense granules. Secreted ATP binds to the cation channel, $\mathrm{P}_{2} \mathrm{X}_{1}$, and $\mathrm{Ca}^{2+}$ enters the cell, which leads to cytoskeletal turnover and shape change. Stimulation of $\mathrm{P}_{2} \mathrm{X}_{1}$ also induces phosphorylation of extracellular signal-regulated kinase 2 (Erk 2), enhancing granule secretion and aggregation. Binding of ADP to the $G_{q}$-coupled $P 2 Y_{1}$ receptor causes PLC $_{\beta 2}$ activation, converting phosphatidyl inositol 4,5-bisphosphate (PIP2) into inositol 1,4,5-trisphosphate (IP3) and diacylglycerol (DAG). IP3 releases $\mathrm{Ca}^{2+}$ from the internal stores, which mediates actin cytoskeletal turnover and changes the shape of the cells. DAG activates protein kinase $C$ (PKC), initiating platelet aggregation. $A D P$ also binds the $G_{i}$-coupled $P 2 Y_{12}$ receptor. The $G_{i \alpha}$ subunit suppresses activity of adenylate cyclase (AC), thereby lowering the level of cyclic AMP (cAMP) and reducing the activity of protein kinase $A(P K A)$. This potentiates the aggregation response. In addition, stimulation of the $\mathrm{P}_{2} \mathrm{Y}_{12}$ receptor activates phosphoinositide 3-kinase (PI3-K), which phosphorylates Akt (PKB) through PIP3, also sustaining platelet aggregation. Adapted from Kahner et al. ${ }^{11}$ 
change response via actin cytoskeleton turnover. ${ }^{17}$ Diacylglycerol activates protein kinase $\mathrm{C}$, thereby triggering one of the key pathways towards platelet aggregation. ${ }^{22}$ The other ADP receptor, $P 2 Y_{12}$, is coupled to the $G_{i}$ protein, which inhibits the effector enzyme adenylate cyclase. ${ }^{23}$ This results in a reduction of the level of cyclic AMP and a suppression of cyclic AMP-dependent kinase (PKA). This relieves the negative regulation of PKA on both $\mathrm{Ca}^{2+}$ signal generation and platelet aggregation. ${ }^{24}$ Another pathway downstream of $G_{i}$ is activation of phosphoinositide 3-kinase (PI 3-kinase), likely the $\beta$ and $Y$ isoforms. ${ }^{25-27}$ Activated PI 3-kinase leads to PKB (Akt) phosphorylation, which is also important for platelet aggregation and dense granule secretion. ${ }^{25,28,29}$

A well-studied paracrine activation mechanism in platelets concerns the $\mathrm{Ca}^{2+}$-dependent release of arachidonate from phospholipids via cytosolic phospholipase $\mathrm{A}_{2}{ }^{30}$ Once this enzyme is activated, it converts the arachidonic acid to prostaglandin $\mathrm{H}_{2}$ and thromboxane $\mathrm{A}_{2}$ via the cytosolic complex, cyclooxygenase/thromboxane synthase. ${ }^{31}$ Secretion of thromboxane $A_{2}$ accelerates platelet activation particularly by ligation to the TPa receptor, which mediates dense granule secretion by coupling to the $G_{q}$ protein. ${ }^{32}$ Thromboxane $A_{2}$ thus also induces mobilization of cytosolic $\mathrm{Ca}^{2+}$, shape change and secretion, ${ }^{33}$ thus enhancing platelet aggregation in the same way as described above.

Together, all these signaling pathways, and others which are not described, evoke conformational changes in the integrins allb $\beta 3$ and $\alpha 2 \beta 1$ (inside-out signaling), which as a consequence bind their respective ligands, fibrinogen and collagen. ${ }^{34}$ Integrin activation is maintained by ADP release, activation of the $\mathrm{P} 2 \mathrm{Y}_{12}$ receptors by ADP and subsequent signaling. ${ }^{27,35}$

A relatively new finding is that the activated integrin $\alpha$ llb $\beta 3$ itself evokes further signaling events to form a stabilized platelet-platelet aggregate. This outside-in signaling pathway is supposed to start with lateral movement and clustering of allb $\beta 3$ in the plasma membrane. ${ }^{36,37}$ Whereas a conformational change of $\alpha$ llb $\beta 3$ is sufficient for high affinity ligand binding, the formation of integrin 'microclusters' is required for outside-in activation of Src and Syk tyrosine kinases (figure 3). Under resting conditions, Src family kinases that are bound to the $\beta 3$ cytoplasmic tail are inactivated by interaction with Csk, but they 


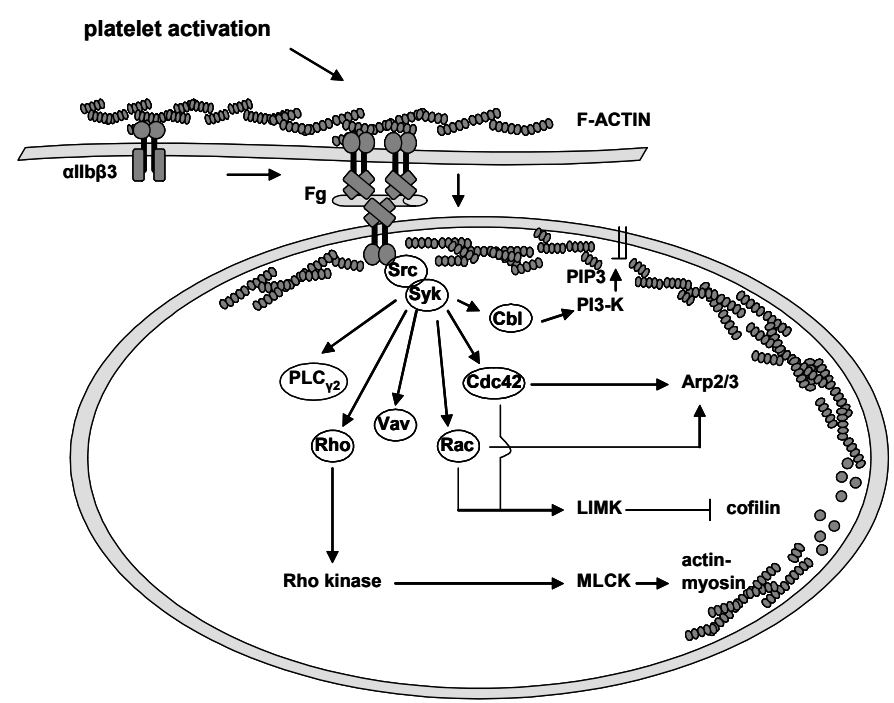

Figure 3. Integrin outside-in signaling pathways involved in actin cytoskeleton reorganization. Agonist-induced activation of platelets results in conformational changes and clustering of the integrin allb $\beta 3$, thus allowing fibrinogen $(\mathrm{Fg})$ binding. In adjacent platelets, this leads to integrin outside-in signaling, in which Src kinase is activated at the $\beta 3$ cytoplasmic tail. This phosphorylates the protein tyrosine kinase Syk, which in turn phosphorylates and activates other signaling proteins like phospholipase $\mathrm{C}_{\gamma^{2}}\left(\mathrm{PLC}_{\gamma_{2}}\right.$ ), small GTPases (Rho, Rac, Cdc42), and the proteins Vav and $\mathrm{Cbl}$. Cbl activates a PI3-kinase (PI3-K), which increases the level of phosphatidylinositol 3,4,5-triphosphate (PIP3) in the plasma membrane. The small GTPase Rho plays a role in stress fiber formation by activating Rho kinase, which phosphorylates myosin light chain kinase (MLCK). Rac inhibits the actin-severing protein cofilin through LIM kinase (LIMK), thereby promoting actin filament growth. It also activates the actin-binding protein, Arp2/3, causing lamellipod formation. Cdc42 plays a role in filopod and lamellipod formation. The precise interactions of the proteins are still investigated.

come in an activated state following fibrinogen binding and clustering of allb $\beta 3$.

The kinase Syk is then recruited and phosphorylates adaptor proteins, small GTPases (like Rac, Rho and Cdc42) and guanine nucleotide exchange factors (like Vav) ${ }^{38-40}$ It is thought that the integrin allbß3 is linked to the actin cytoskeleton via these proteins. ${ }^{41,42}$ Activation of Rac, Rho and Cdc42 is essential for cytoskeletal reorganization and the development of filopodia, lamellipodia and stress fibers. ${ }^{43}$ These morphological changes are implicated in platelet shape change and spreading. ${ }^{44}$ Another protein that is phosphorylated by Syk is the adaptor protein $\mathrm{Cbl}$, which links integrin signaling to the PI 3kinase route, also playing a role in the spreading of platelets on fibrinogen. ${ }^{45,46}$

Shedding of microparticles occurs after activation of platelets with strong agonists, like collagen and thrombin. The mechanism of this formation of 
microparticles is not fully understood. It has been proposed that the $\mathrm{Ca}^{2+}$ dependent protease calpain is involved, for instance by cleaving actinassociated proteins and disrupting the integrity of the actin cytoskeleton. ${ }^{47}$ It has also been suggested that microparticles are formed by allb $\beta 3$ signaling towards actin cytoskeleton destabilization, possibly involving the Wiskott-Aldrich syndrome protein (WASP). ${ }^{48,49}$

\section{Platelet activation and coagulation}

Activated platelets play a key role in blood coagulation. Coagulation is considered to start with the binding of vascular (or blood-borne) tissue factor to the protease, factor VIla. ${ }^{50}$ This complex mediates further cleavage and activation of trace amounts of coagulation factors, such as factors IX and $X .^{51,52}$ Resting platelets do not support this process, as the procoagulant aminophospholipids, phosphatidylserine (PS) and phosphatidylethanolamine $(P E)$, are exclusively located at the cytoplasmic side of the plasma membrane. However, upon platelet activation, these phospholipids, particularly negatively charged PS, are exposed at the outer surface of platelets via a hitherto unidentified scramblase. ${ }^{51,53}$ The PS-exposing platelets provide an excellent membrane surface for $\mathrm{Ca}^{2+}$-dependent assembly of coagulation factor activating complexes, such as the tenase complex (consisting of factors VIIla, IXa and X) and the prothrombinase complex (consisting of factors $\mathrm{Xa}, \mathrm{Va}$ and prothrombin). Together, these complexes greatly enhance the generation of factor $\mathrm{Xa}$ and thrombin. ${ }^{54}$ Since activated platelets shed microparticles, which also have exposed PS, the formation of factor $\mathrm{Xa}$ and thrombin becomes even more enhanced. Thrombin converts fibrinogen into fibrin, thus resulting in the formation of a stable thrombus consisting of platelets and fibrin (figure 1).

The activation steps in the coagulation cascade are inhibited by anticoagulant proteins. For instance, tissue factor pathway inhibitor binds free factor $\mathrm{Xa}$ and subsequently inactivates membrane bound tissue factor/factor VIla complex. ${ }^{55}$ Activated protein $\mathrm{C}$ together with its cofactor, protein $\mathrm{S}$, inactivate factor Va and factor VIIla, which in turn limits the production of thrombin. ${ }^{56,57}$ 
Anti-thrombin directly inhibits thrombin and other activated coagulation factors, altogether limiting thrombus formation. ${ }^{58}$

\section{Platelet transfusion therapy}

Under normal conditions, platelets circulate in the blood at concentrations of $150-400 \times 10^{9} / \mathrm{L}$. In thrombocytopenia, the platelet count is greatly reduced due to inadequate platelet production from the bone marrow or to increased platelet removal in spleen, liver or vessel wall. Patients are considered to be thrombocytopenic when their platelet concentration drops below $80-100 \times 10^{9} / \mathrm{L}$. However, the threshold for a life-threatening risk of (spontaneous) bleeding is much lower, namely $20 \times 10^{9}$ platelets/L. In current clinical practice in the Netherlands, platelet transfusions are given at counts $<50 \times 10^{9}$ platelets $/ L$ in case a bleeding needs to be arrested and, prophylactically, at counts $<10 \times 10^{9}$ platelets/L to prevent bleeding. ${ }^{59,60}$ Today, platelet transfusion has become a mainstream support therapy for cancer patients undergoing bone marrow transplantation, in whom the normal platelet production is suppressed by cytostatics. $^{61,62}$ Less frequently, platelet transfusion is given in case of thrombocytopenia due to the presence of auto-immune or allo-immune antibodies (i.e. HPA, HLA or the blood group antigens, ABO), or to patients suffering from drug-induced platelet consumption. ${ }^{63}$ Another indication for platelet transfusion is thrombocytopenia due to major invasive surgery or major trauma. ${ }^{64}$

The most common adverse effects of transfusion with platelet preparations are allergic reactions and fever, although these usually do not form a clinical problem. ${ }^{65}$ Less common but more problematic is the risk of immunization with allo-antigens. Patients who have antibodies against HPA or HLA need to be transfused with antigen-compatible platelet preparations. Transfusion with an incompatible (or unscreened) preparation can result in agglutination of the transfused platelets and, hence, a higher risk of bleeding after treatment. Other rare, but yet significant clinical side effects are transfusion-associated graftversus-host disease, acute lung injury and immune hemolytic reactions. ${ }^{66,67}$ Relevant acquired transfusion risks are bacterial or viral infections, e.g. with 
cytomegalovirus or human immunodeficiency virus.

During the last 50 years, blood banks have developed strictly controlled procedures of platelet preparation and storage to optimize platelet 'quality' and to reduce the risk of infection. For the Netherlands, these are described in the Blood Transfusion Guidelines from the Dutch Institute for Healthcare. ${ }^{68}$ The prescriptions include that platelet preparations are kept at room temperature in gas-permeable transfusion bags under continuous lateral movement. ${ }^{69}$ The platelet products need to be filtered before storage to remove bacterial contaminants and reduce leukocytes and, hence, prevent allo-immunization. ${ }^{70}$ Before transfusion, platelets must be tested for absence of undesired microorganisms and viruses. Stored platelets may be irradiated, e.g. to prevent graftversus-host disease, before delivery to susceptible patients. However, despite all these procedures and precautions, still a considerable number of patients, particularly those undergoing multiple blood transfusions, become refractory to donated platelets, in which cases HPA/HLA-matched preparations need to be given. ${ }^{71}$

After the transfusion intervention in patients, a substantial part of the donor platelets is cleared from the circulation after only a few passages. After 1 hour the survival percentage of the transfused platelets is mostly $<60 \% .{ }^{72}$ Commonly, the success of a platelet transfusion is judged from the increase in platelet count 1 or 24 hours after the intervention. Data are expressed as the so-called corrected count increment, which is a parameter correcting for the body mass (i.e. blood volume) of the patient. Yet, this measure appears to provide little information on the bleeding risk of the patient. ${ }^{73}$

\section{Platelet preparation and storage}

In blood banks in the Netherlands and other European countries, platelet preparations are currently made according to two different procedures. Single donor preparations are prepared by the apheresis method, where anticoagulated whole blood is separated into platelets, plasma and red cells during the donation (figure 4A). After this separation, achieved by bedside centrifugation, the red cells are returned to the donor. The platelet and plasma 
fractions are combined to give a platelet-rich plasma preparation $\left(\sim 250 \times 10^{9}\right.$ platelets in $150-400 \mathrm{~mL}$ plasma), which is depleted from leukocytes by filtration or another leukoreduction system to result in $<1 \times 10^{6}$ leukocytes per sample. This platelet preparation can be stored for up to 5 days at $22^{\circ} \mathrm{C}$ in a lateral shaking device. ${ }^{69,74}$ In the Netherlands, such apheresis platelet preparations are usually prepared for transfusion to otherwise refractory patients, e.g. with antibodies against human platelet antigens (HPA) or human lymphocytic antigens (HLA). About $5-10 \%$ of the platelets are produced by apheresis.

The standard procedure to prepare platelets is by centrifugation of donated whole blood (figure 4B). The anti-coagulated blood needs to be centrifuged within $24 \mathrm{~h}$ after donation. The resulting buffy coat, containing platelets and leukocytes, is physically separated from red cells and plasma. Buffy coats from five different donors are then pooled and supplemented with plasma of one of these donors. The mixture is leukocyte-reduced by filtration. ${ }^{69,74}$ The remaining product ( $250 \times 10^{9}$ platelets and $<1 \times 10^{6}$ leukocytes per batch) is stored up to 7 days under the same conditions as apheresis platelets.
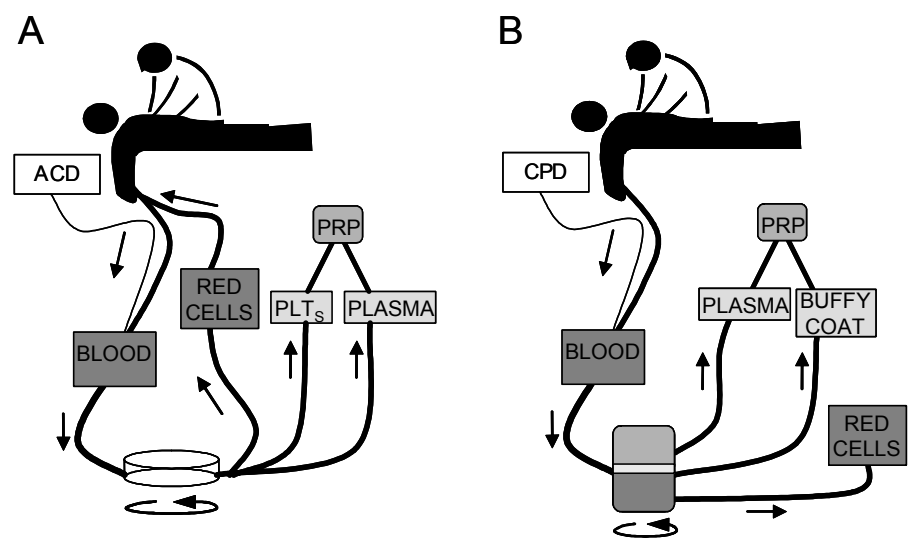

Figure 4. Methods to generate platelet preparations. (A) Apheresis method: acid-citrate-dextrose (ACD) anti-coagulated whole blood is centrifuged during donation, and red cells are immediately returned to the donor. Donor platelets and plasma are pooled to generate a platelet-rich plasma (PRP) preparation. (B) Buffy coat method: citrate-phosphate-dextrose (CPD) anti-coagulated whole blood is centrifuged after donation to separate red cells, plasma and buffy coat. Buffy coats (containing platelets) from five different donors are pooled with the plasma of one of these donors to generate PRP. Before storage, both types of PRP preparations are filtered to remove leukocytes. 
In the last few years, there is a tendency at blood banks to store platelet preparations in solutions with only $30 \%$ plasma. Advocated advantages of storage in such synthetic media are that plasma is saved for other purposes and that transfusion side reactions are reduced, because of diminished exposure of the patient to donor material. ${ }^{75}$ However, storage in synthetic medium may alter the function of the stored platelets and reduce the transfusion efficacy of the cells. ${ }^{76,77}$ For instance, replacement of plasma by synthetic medium can result in increased expression of activation markers at the platelet surface. ${ }^{78,79}$

In blood banks, the quality of platelet preparations is routinely monitored by assessing the platelet count, by measuring the $\mathrm{pH}$ (normal range $\mathrm{pH} 6.8$ to 7.4), and by testing the capability of the cells to 'swirl' (absence of shape change). For specific purposes, platelet quality is evaluated more rigorously from the morphology (absence of pseudopods), the so-called hypotonic shock response (i.e. resistance of the cells to dilution with water), the extent of shape change, the production of lactate, or overall metabolic activity. ${ }^{80}$ Since the results are often unrelated to each other, it is still debated which of these tests is preferred as primary quality marker. ${ }^{81} \mathrm{~A}$ clear limitation of these tests is that they are only distantly related to the physiological properties of platelets in hemostasis.

\section{Aims and outline of this thesis}

The research presented in this thesis firstly examines which alterations platelets undergo during storage and how these can be explained by changes in signal transduction processes. In chapter 2 an overview is given of the current understanding of the causes of platelet storage lesion, i.e. the gradually increasing functional defect of stored platelets. This chapter mainly reviews the earlier literature, but also refers to some of the new data described in this thesis. Chapter 3 starts from the hypothesis that platelets become activated during their storage, while their responsiveness towards agonists decreases. It is assumed that the alterations in platelet function are caused by purinergic receptor desensitization, due to a continuous slow release of autocrine ADP and ATP, reducing the responsiveness towards these agonists. A novel finding 
is that stored platelets are protected from such desensitization, because plasma contains a high activity of ADP- and ATP-degrading enzymes. This discovery has led to the research presented in chapter 4 . Here, the function of platelets that are stored in plasma is compared to the function of platelets stored in synthetic media. An earlier finding was that preparations of stored platelets contain large amounts of procoagulant microparticles. In chapter $\mathbf{5}$, it is investigated how these microparticles are formed. The experiments considered the involvement of purinergic receptors, integrin allb $\beta 3$ and the actin cytoskeleton. The impairment of signaling processes in platelet storage lesion is further examined in chapter 6 .

The second goal of this thesis is the development of tests to assess improved platelet functionality after transfusion. Chapter 7 describes the development of two methods which report on the properties of platelets in patient plasma, namely flow-dependent thrombus formation and thrombin generation. Using these methods, the effects of transfusion with platelets stored in full plasma or in synthetic medium are compared. Attention is paid to the consequences of a decreased in vitro function of stored platelets for the hemostatic properties post-transfusion. Finally, the principal findings of this thesis are discussed in chapter 8, with attention to their relevance for blood bank practice and transfusion medicine. 


\title{
Chapter 2
}

\section{Hemostatic and signaling functions of transfused platelets}

\author{
Sandra Cauwenberghs, Elisabeth van Pampus, \\ Joyce Curvers, Jan-Willem N. Akkerman and \\ Johan W.M. Heemskerk
}

Transfusion Medicine Reviews

2007 (in press)

Reprinted with permission 


\begin{abstract}
Metabolic studies have revealed a gradual impairment in platelet integrity during storage, a process termed the platelet storage lesion. Recent evidence shows that stored platelets also loose signaling responses to physiological agonists with impaired integrin activation, secretion and aggregation of the cells. On the other hand, storage leads to a gain in platelet activation properties, such as release of microparticles and appearance of surface epitopes for their clearance by macrophages. New techniques for measuring flow-induced thrombus formation and platelet-dependent coagulation provide evidence that the hemostatic activity of platelets decreases during storage. Besides pharmacological inhibition, novel storage strategies, like metabolic suppression, should be considered to better preserve platelet functionality, while limiting the expression of clearance markers. Understanding the changes that occur in association with the platelet storage lesion and the use of updated storage methods will help to generate platelets for transfusion with optimal hemostatic function and a long circulation time after transfusion.
\end{abstract}




\section{Introduction}

Platelets play a critical role in hemostasis. Indeed, hemorrhages can be caused by both quantitative and qualitative platelet disorders. Therapeutically, platelet concentrates are used for transfusion to thrombocytopenic patients with bleeding. In addition, these concentrates are increasingly being used prophylactically to prevent bleeding in patients with thrombocytopenia due to chemotherapy treatment. However, the shelf life of stored platelet concentrates is limited to 5-7 days for a variety of reasons. In this report, we give an overview of the current insights of the functional alterations in stored platelets and the possible consequences for platelet transfusion therapy.

\section{Effective platelet transfusion}

The purpose of transfusion to thrombocytopenic patients is to supply a sufficient number of donor cells with hemostatic properties similar to those of the patient's own platelets. This implies that upon vascular damage or rupture, donor platelets need to change from resting, non-adhesive cells to highly reactive and sticky elements. In addition, donor platelets are supposed to remain in the circulation for a normal period of time. Thus, the ideal transfusion concentrate contains platelets with a low activation state, a high activation tendency, and the absence of markers of their destruction and removal.

In early studies, the hemostatic activity of transfused platelets was deduced from their ability to shorten the skin bleeding time, but this technique appeared to be relatively insensitive. ${ }^{82}$ More refined techniques, based on transfusion of (radio)labeled platelets and analysis of platelet count increment, revealed a marked reduction in the circulation time, when stored platelets are compared to fresh platelets. The life span even declined as storage of the platelets was longer. ${ }^{83,84}$ Explanations for this reduced platelet survival can be sought in immunological reactions (e.g. post-transfusion thrombocytopenia purpura) or in altered surface characteristics that trigger platelet clearance ${ }^{85,86}$ However, the current literature provides substantial evidence which indicates that changes in functional properties also affect platelet survival. 


\section{Platelet responses in hemostasis}

Insight into the processes of thrombus formation following vascular injury has increased considerably by the availability of experimental thrombosis models and genetically modified mice. ${ }^{12,87}$ In addition, ex vivo flow chamber studies with murine and human blood have made it possible to unravel intracellular activation pathways that trigger thrombus formation under physiologically relevant conditions. ${ }^{88-90}$ Exposure of collagen fibers leads to platelet arrest and activation via an interplay of adhesive receptors (figure 1). Together with glycoprotein (GP) lb $\mathrm{lb}$, which enables reversible attachment to collagen-bound von Willebrand factor (VWF) and slows down the circulating platelets, the adhesive receptor integrin $\alpha 2 \beta 1$ and the signaling receptor GPVI jointly initiate firm adhesion on and activation of platelets by collagen., ${ }^{9,91,92}$ Subsequent platelet responses include $\mathrm{Ca}^{2+}$ mobilization, inside-out activation of integrin $\alpha$ llb $\beta 3$ and fibrinogen binding, pseudopod formation, shape change, and thromboxane $\mathrm{A}_{2}$ formation. Another key response is exocytosis as the result of granule fusion, causing expression of CD62 (P-selectin) at the platelet surface and release of the paracrine agents ADP, ATP and Gas6. ${ }^{12,27}$ All these processes together mediate the formation of platelet aggregates. In a final stage of activation, the platelets also express procoagulant phosphatidylserine at their outer membrane surface, which is required for optimal function of the coagulation process. At this stage, microparticles are formed which further contribute to platelet procoagulant activity. ${ }^{5,93}$ Thrombin, generated on the surface of both procoagulant platelets and microparticles, amplifies platelet activation and cleaves fibrinogen to form fibrin, thus stabilizing the platelet aggregate to a clot. ${ }^{87,94}$ Finally, platelet-dependent clot retraction is required for stable plug formation and persistent arrest of bleeding.

\section{Testing of stored platelet peparations}

Investigations to preserve the quality of stored platelets initially focused on their metabolism and energy state. Platelet concentrates kept in anti-coagulated plasma were supplemented with glucose to maintain a high intracellular ATP/ADP ratio. 


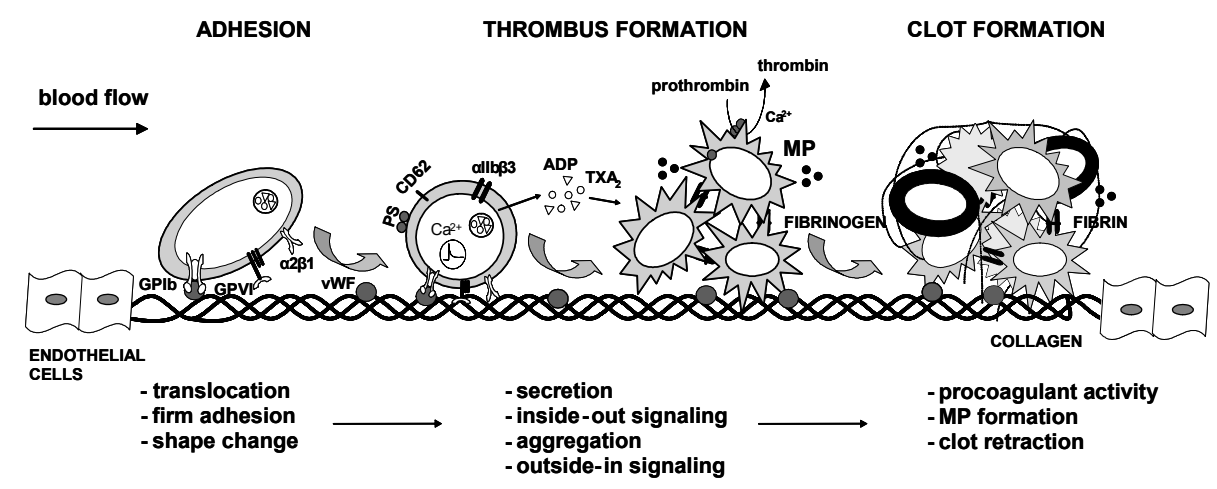

Figure 1. Schematic overview of platelet responses in thrombus and clot formation. Sheardependent translocation of platelets over exposed vWF/collagen results in adhesion, initially via GPIb-V-IX, and subsequently via GPVI and integrin $\alpha 2 \beta 1$. Shape change (pseudopod formation and loss of discoid morphology) stabilizes the adhesion, while intracellular signaling leads to mobilization of $\mathrm{Ca}^{2+}$ from internal stores, and subsequent release of paracrine agents, such as ADP, ATP and thromboxane $A_{2}\left(T X A_{2}\right)$. The released products activate other platelets via $G-$ protein coupled receptors. Platelets respond by inside-out signaling and activation of the fibrinogen receptor, allb $\beta 3$. Aggregates are formed by platelet-platelet interactions via fibrinogen and vWF. Activated allb $\beta 3$ also signals outside-in to reorganization of the actin cytoskeleton and spreading of platelets. Rises in cytosolic $\mathrm{Ca}^{2+}$ furthermore cause platelets to develop a procoagulant phosphatidylserine (PS)-exposing surface, and to shed procoagulant microparticles (MP). Thrombin, produced from prothrombin on the procoagulant platelet/microparticle surface, acts as a potent platelet agonist and also transforms soluble fibrinogen into a fibrin network. Fibrin clot formation stabilizes the growing thrombus, and is followed by platelet-dependent clot retraction.

Air-transparent bags were introduced to reduce anaerobic lactate production and preserve a neutral plasma $\mathrm{pH}^{95}$ Metabolic markers of stored platelet concentrates, e.g. $\mathrm{pCO}_{2}, \mathrm{pO}_{2}, \mathrm{pH}$, lactate and glucose, still are widely used quality parameters. Platelet integrity is often deduced from tests measuring general cellular (dys)function, such as mean platelet volume (MPV), hypotonic shock response (HSR), extent of shape change (ESC), and the 'swirling' reaction. ${ }^{96}$ Indeed, for practical reasons, quality checks of stored platelets are often restricted to these conventional assays, even though it has become clear that they reflect properties that are only indirectly relevant for the hemostatic function of platelets. Rather, they detect a substantial loss of cell integrity or a general loss of platelet function during prolonged storage. Originally the term platelet storage lesion was used to indicate the loss of platelet integrity or platelet function in general. However, it is now recognized that platelets can loose or gain several of their functions, even when stored in native plasma. 


\section{A. ADHESION / THROMBUS FORMATION}
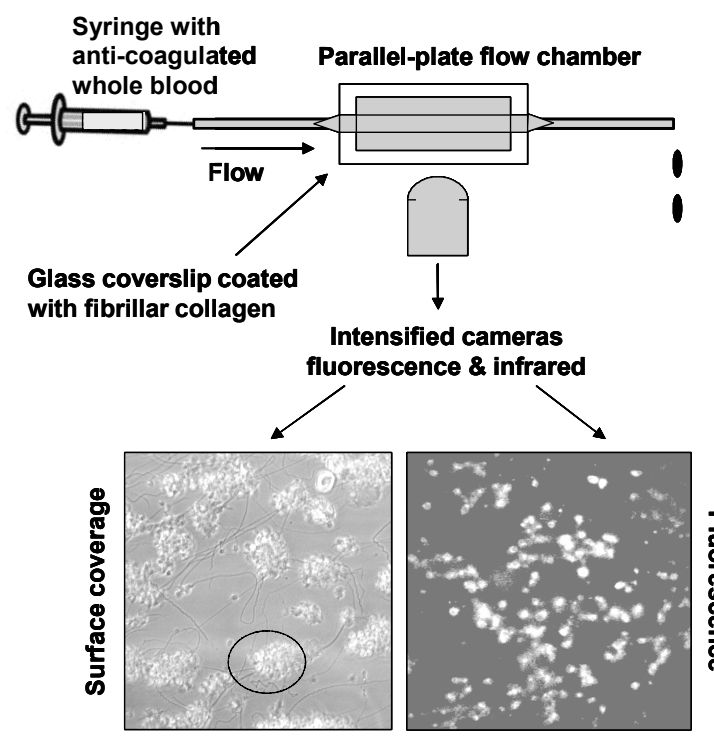

\section{B. CLOT FORMATION}
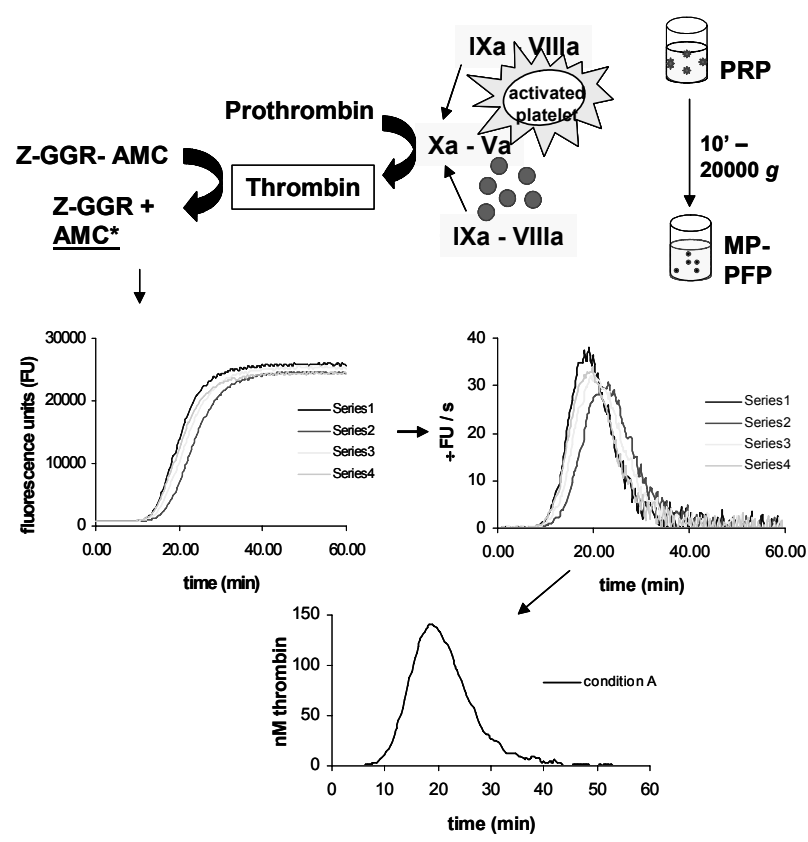

Figure 2. Platelet function tests to evaluate hemostatic activity at normal and low platelet counts.

Panel A. Top: Whole blood perfusion test to measure platelet adhesion and thrombus formation. Schematic setup of parallel-plate flow chamber (with platelet-adhesive collagen surface), connected to a pulse-free pump with syringe and a fluorescence microscope with intensified cameras. Bottom: Phase-contrast image of thrombi and image of fluorescentstaining after perfusion of whole blood with normal platelet count (4 minutes at $1000 / \mathrm{s}$, bar $=50 \mu \mathrm{m})$.

Panel B. Thrombin generation assay to measure procoagulant activity of platelet- or microparticle (MP) containing plasma. Top: Procoagulant platelets and MP, which catalyze the formation of factor $\mathrm{Xa}$ by the tenase complex (factors VIIla and IXa) and of thrombin by the prothrombinase complex (factors $\mathrm{Va}$ and $\mathrm{Xa}$ ). The formed thrombin cleaves Z-Gly-Gly-Arg aminomethyl coumarin (Z-GGR-AMC), which is measured in the thrombin generation assay. Bottom: The increase in fluorescence from cleaved $\mathrm{AMC}^{*}$ is measured continuously (excitation wavelength $390 \mathrm{~nm}$ and emission $460 \mathrm{~nm}$ ) in platelet-rich plasma. Firstderivative curves (dFU/s) are calculated from the change in fluorescence, and converted into thrombin generation (nM) curves. ${ }^{94}$ For application in thrombocytopenia, see Ref. ${ }^{97}$ 
In recent years, the testing of platelet function has shifted to the assessment of signaling processes and activation properties relevant to hemostasis. Flow cytometry is used to detect surface alterations reflecting platelet activation such as integrin activation, secretion of granule contents and phosphatidylserine exposure. $^{98}$ There is a rapid increase in the use of platelet adhesion, aggregation and platelet-dependent coagulation assays for determining loss of function during storage. ${ }^{99,100}$ This research has shown that platelet storage lesion affects many different functions of the cell, but there is still little insight into the impact of each of these changes on the hemostatic behavior after platelet transfusion. The main reason is the thrombocytopenia of transfused patients, which has made it difficult to assess platelet function with tests other than flow cytometry.

Therefore, new technologies are being developed to study activation processes at low platelet counts. Promising tools are cone-and-plate devices and flow chambers for the analysis of shear-induced aggregation and thrombus formation. ${ }^{90,91,101}$ One of the recently described flow chamber systems quantifies thrombus formation after the perfusion of anti-coagulated thrombocytopenic blood over a collagen-coated surface at defined shear conditions (figure $2 \mathrm{~A}$ ). ${ }^{97}$ Another suitable test is the measurement of thrombin generation in thrombocytopenic plasma. In the calibrated automated thrombogram method, platelets are activated to provide a procoagulant surface, which facilitates the formation of thrombin. ${ }^{94,102}$ The protease activity of thrombin is continuously measured by cleavage of a fluorescent thrombin substrate (figure 2B). Even at low platelet counts, this method senses the procoagulant response of platelets. ${ }^{97}$ These techniques are likely to provide important information on the hemostatic function of platelets after transfusion.

\section{Platelet function associated with the storage lesion}

After storage of platelets, their stable adhesion to adhesive surfaces is shown to be diminished (table 1). Diminished adhesion to vWF under shear is probably caused by structural changes to the vWF receptor complex, GPIb-VIX. Platelets release metalloproteinases, which cleave the GPIba and GPV 
chains of this complex, and alter their adhesive function. ${ }^{103-105}$ Other investigators report that platelet storage leads to clustering of GPIb-V-IX receptors, which upon transfusion enhances clearance of platelets from the circulation. ${ }^{86,106}$ Thus there are a number of ways, in which prolonged storage can affect stable adhesion and the ensuing aggregation of platelets on a VWFcollagen surface. ${ }^{99,100}$

Loss in disc shape morphology and a small increase in basal $\mathrm{Ca}^{2+}$ level point to alterations in $\mathrm{Ca}^{2+}$ handling during storage of platelets. ${ }^{96,107}$ In addition, storage compromises signaling to $\mathrm{Ca}^{2+}$ mobilization by receptors coupled to $\mathrm{G}$ proteins (epinephrine, ADP, thromboxane $\mathrm{A}_{2}$ and thrombin receptors) or to tyrosine kinases (collagen receptors). ${ }^{100,108}$ This illustrates a reduced tendency of stored platelets to become activated, which is supported by analysis of tyrosine phosphorylation patterns, showing diminished thrombin-induced phosphorylation of multiple bands upon storage. ${ }^{109}$ Furthermore, agonistinduced secretion, as deduced from the surface exposure of granular CD62 ( $P$ selectin), decreases with storage time. ${ }^{100,110}$

Typically, this fall in activation responses after agonist stimulation is accompanied by an increase in surface expression of platelet activation markers, suggesting that the activation state of platelets increases during storage. Initially, there is a slow exposure of CD62 and a mild activation of integrin allbß3 accompanied by actin cytoskeletal reorganization. ${ }^{111}$ At a later stage, stored platelets begin to expose phosphatidylserine, which makes the cells procoagulant. ${ }^{100}$ However, phosphatidylserine exposure is also a marker for in vivo scavenging. ${ }^{112,113}$ The procoagulant activity of stored platelets is further stimulated by the gradual shedding of procoagulant microparticles, which appears to be linked to allbß3-mediated cytoskeletal reorganization. ${ }^{114}$

The platelet purinergic receptors, $P 2 Y_{1}$ and $P 2 Y_{12}$ for $A D P$, and $P 2 X_{1}$ for ATP, are prone to desensitization via autocrine activation mechanisms. ${ }^{115}$ At first sight, this may explain the gradual impairment of ADP-induced integrin activation and aggregation during platelet storage. Recent evidence shows that platelets that are kept in plasma resist ADP and ATP receptor desensitization due to the presence of soluble ecto-nucleotidases, which degrade released 
ADP and ATP. ${ }^{116}$ This is relevant for the use of platelet-additive solutions, because a reduced content of plasma increases purinergic receptor desensitization and, hence, decreases in vitro aggregation responses. ${ }^{78}$

Integrin allbß3 activation and ligand binding trigger so-called outside-in signaling responses, such as platelet spreading via protein tyrosine kinases (Src and focal adhesion kinases). ${ }^{37}$ Another outside-in allb $\beta 3$ signaling event is retraction of platelet-containing clots, which occurs in a late stage of coagulation. Platelet clot retraction in concentrates increases with the storage time, which is an indication that allbß3 signaling increases upon storage. ${ }^{117,118}$ In summary, the current literature points to a dual effect of storage on hemostatic platelet properties; appearance of activation-like processes (surface activation markers and integrin signaling) and reduced responsiveness towards agonists (diminished adhesion, secretion and aggregation). The platelet storage lesion thus produces platelets with an increased activation state and a decreased activation tendency.

\section{The storage lesion and platelet clearance}

It is reported that platelet storage for more than 48 hours significantly reduces the beneficial effect of platelet transfusion in thrombocytopenic patients. Prolonged storage shortens the circulation time of the transfused cells, likely due to cell surface changes. ${ }^{67}$ The expression of CD62 and phosphatidylserine leads to platelet recognition by macrophages and subsequent clearance from the circulation. ${ }^{86}$ Other surface changes may also be involved, such as the state of activated integrins ( $\alpha$ llb $\beta 3$ ) and the presence of pseudopodia. ${ }^{111}$ Storage at lower temperature $\left(4^{\circ} \mathrm{C}\right)$ in particular induces clustering of GPIb-V-IX, which generates recognition epitopes for the $\alpha M \beta 2$ integrin (Mac-1) of macrophages and, thereby, enhances the removal of platelets from the circulation. ${ }^{106}$ Unlike CD62-dependent removal, clearance via $\alpha \mathrm{M} \beta 2$ is a rather slow process, ${ }^{86}$ but efforts have been made to reduce this process. In mice, modification of GPIba by galactysolation of its $\beta-\mathrm{N}$-acetyl glucosamine residues impairs recognition by macrophages, and this prolongs 
Table 1. Overview of functions affected in platelet storage lesion.

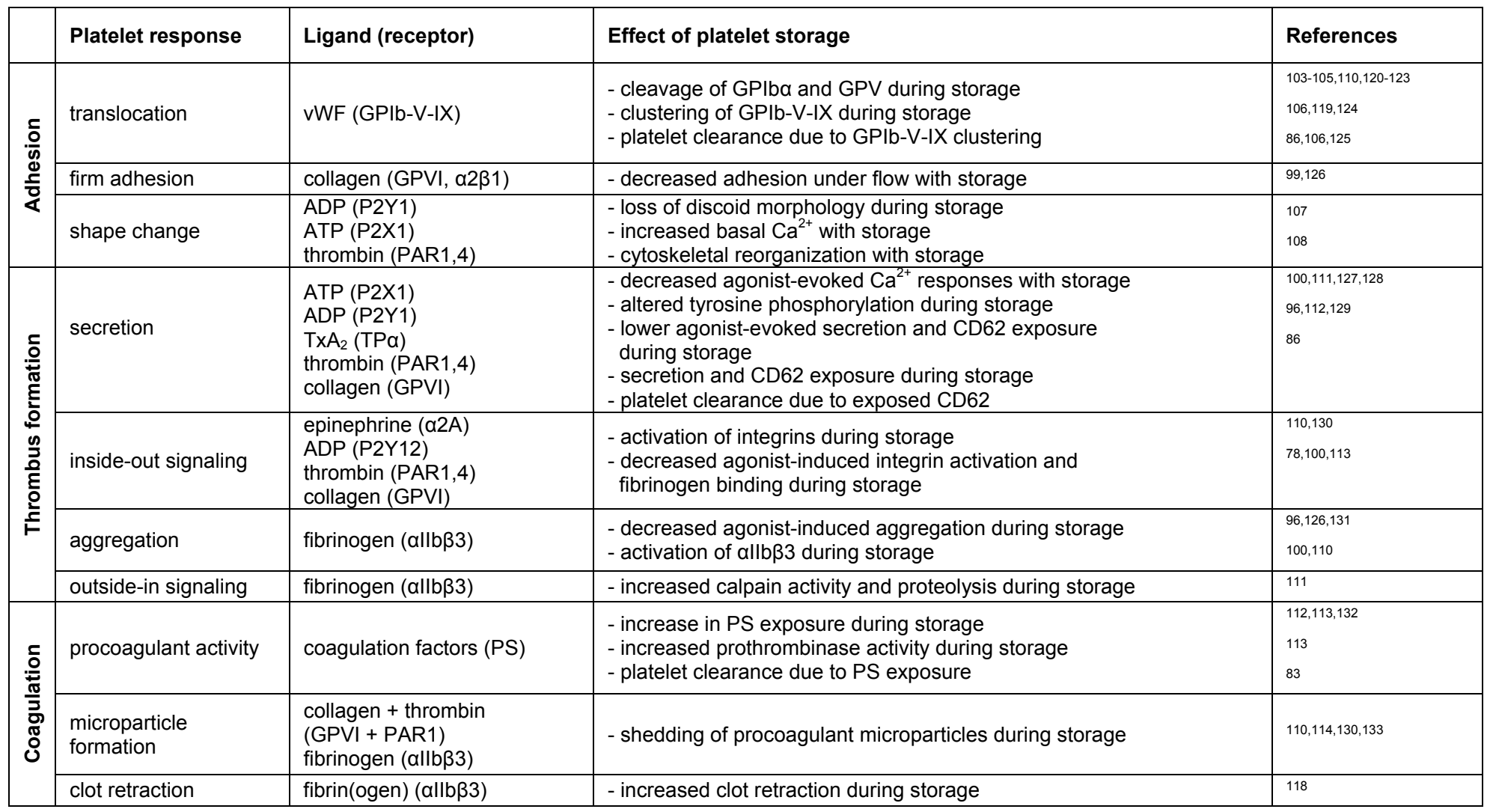


the circulation time of cold-stored platelets. ${ }^{106,119}$ However, a recent publication shows that this is not the case for galactosylation of human cold-stored platelets, ${ }^{134}$ suggesting a joint contribution of surface markers in the clearance of platelets after transfusion. As discussed above, the GPIb-V-IX complex can also be affected by cleavage by matrix metalloproteinases. Again in mice, inhibition of the metalloproteinase ADAM17 results in higher post-transfusion platelet recovery and improved hemostatic function. ${ }^{125}$ Prevention of storageinduced surface changes by using platelet inhibitors, e.g. prostacyclin or nitric oxide, may provide another way to prolong the platelet circulation time. ${ }^{135}$

Typically, the interaction of GPIb-V-IX with VWF - which is assessed by measurement of the GPIb-dependent ristocetin cofactor activity of platelets decreases only slightly upon storage. ${ }^{105}$ Furthermore, vWF multimers in plasma do not appear to be degraded, suggesting that matrix metalloproteinase activity in stored platelet concentrates is low.

\section{Platelet recovery after transfusion}

There are some indications that platelet storage defects can be restored after their transfusion to patients. Platelet count measurements will not inform on secondary recovery of platelet function. However, various ex vivo tests have been applied to investigate post-transfusion adhesion and aggregation responses, such as adhesion to collagen-coated beads. ${ }^{126,131}$ Flow experiments, measuring collagen- and ADP-dependent thrombus formation under shear, provide indications of increased activation via GPVI and P2Y receptors after platelet transfusion to patients. ${ }^{97}$

\section{Suppression of platelet storage lesion by metabolic arrest}

Previous attempts to limit the platelet storage lesion were based on pharmacologic inhibition, e.g. (a) using agents which increase cyclic AMP levels and (b) nitric oxide-donating compounds. ${ }^{136-138}$ Although some progress was made, difficulties to remove these agents prior to transfusion have seriously hampered their widespread application. In an alternative approach, metabolic 
arrest has been used to interfere with the development of platelet changes during storage. Earlier observations had shown that platelets can survive a period of energy shortage in glucose-free medium in the presence of the inhibitor of mitochondrial ATP resynthesis, antimycin A. ${ }^{139}$ At $37^{\circ} \mathrm{C}$, this leads to a fall in adenylate energy charge of the metabolic compartment, which is expressed as the ratio (ATP + $1 / 2$ ADP) / (ATP + ADP + AMP). This parameter depicts the balance between energy production and consumption, and is a sensitive marker for the energy status of the cell. The fall in adenylate energy charge is accompanied by a gradual loss of the capacity of platelets to secrete and aggregate. Restoration of the adenylate energy charge by one hour incubation in excess glucose restores platelet function. ${ }^{140,141}$

In comparison to platelets stored under normal conditions, the metabolically suppressed platelets remain low in surface CD62 expression, but still lose their aggregability upon storage. ${ }^{142}$ The outcome of this approach improves dramatically, when the energy deprivation is followed by storage at $4^{\circ} \mathrm{C}$. First, the platelets are energy-depleted by incubation at $37^{\circ} \mathrm{C}$ in glucose-free, antimycin $\mathrm{A}$ containing buffer, which makes the cells inactive. Then, the platelet suspensions are cooled down to $4^{\circ} \mathrm{C}$ and stored for up to 48 hours. When rewarmed in glucose-rich medium, CD62 expression remains low, while thrombin receptor-induced aggregation recovers substantially. The cold-stored, metabolically suppressed platelets also preserve most of their capacity to adhere to a VWF- or fibrinogen-coated surface in a flow chamber. ${ }^{142}$

A drawback of such an approach to the cooling of platelets is that they tend to expose epitopes that are recognized by macrophages, triggering their removal from the circulation (see above). Thus although platelet functions might be better preserved following energy depletion and cold storage, after transfusion the platelets would rapidly disappear from the circulation with little benefit to the thrombocytopenic patient. An in vitro phagocytosis assay, based on co-incubation of platelets with mature monocytic cells, shows that the elimination of stored platelets can be separated into three phases. ${ }^{143,144}$ First, there is the binding of platelets to monocytes, which is in part mediated by platelet CD62 with P-selectin glycoprotein ligand-1 (PSGL-1) as counter- 
receptor on the monocytes. Second, there is the expression of phosphatidylserine on the stored platelets, which triggers phagocytosis via scavenging receptors. Third, there is the clustering of GPIb-V-IX complex that accompanies cooling, probably caused by disturbances in surface sugar chains. The clustered GPIba molecules are recognized by integrin $\alpha \mathrm{M} \beta 2$ receptors on the monocytes, again causing phagocytosis. ${ }^{106}$ Metabolic deprivation of platelets has little impact on their binding to monocytes, but suppresses the expression of markers of phagocytosis and, hence, the phagocytic process. Furthermore, the expression of phagocytosis epitopes appears to be energydependent, as storage of the metabolic deprived platelets at $4^{\circ} \mathrm{C}$ prevents an increase in phagocytosis due to the cold temperature. ${ }^{144}$

\section{Concluding remarks}

Conventional methods for the assessment of the quality of stored platelets mostly inform on the state of their general (dys)function. It now appears that the platelet storage lesion is a change that extends to most physiological functions of platelets. Novel technologies assessing the platelet signaling state should be used to better define the responses of stored platelets that are relevant for hemostasis. These approaches can help with the development of improved ways of storage to obtain platelets that are fully active and do not express phagocytosis epitopes. Thus the addition of natural platelet-inhibiting compounds like prostacyclin, nitric oxide and the ecto-nucleotidase CD39, degrading ADP and ATP, could reduce the expression of platelet activation and phagocytosis markers. Metabolic suppression at $4^{\circ} \mathrm{C}$ is another promising tool to possibly extend the storage time of platelets for transfusion with minimal effects on platelet activation and circulation. Unfortunately, we currently lack a standardized thrombocytopenic animal model, relevant to the human situation, with (autologous) stored platelets as a defined variable, to investigate how platelet activity, survival and phagocytic clearance jointly contribute to the pro-hemostatic effect of a platelet transfusion.

Acknowledgment: This work was partially financed by the Sanquin Blood Supply Foundation (PPO 01.019). 



\title{
Chapter 3
}

\section{Plasma ecto-nucleotidases prevent desensitization of purinergic receptors in stored platelets}

\author{
importance for platelet activity \\ during thrombus formation
Sandra Cauwenberghs, Marion A.H. Feijge,
Geja Hageman, Marc Hoylaerts,
Jan-Willem N. Akkerman, Joyce Curvers
and Johan W.M. Heemskerk

Transfusion

2006; 46:1018-28

Reprinted with permission 


\section{Abstract}

Background: Platelets contain purinergic receptors for ATP $\left(\mathrm{P}_{2} \mathrm{X}_{1}\right)$ and ADP $\left(P 2 Y_{1}\right.$ and $\left.P 2 Y_{12}\right)$ that rapidly desensitize upon stimulation with these nucleotides. In vivo, this is antagonized by ecto-nucleotidases on the surface of endothelial cells and white blood cells (WBCs). The receptor desensitization of ATP- and ADP-induced responses of platelets stored in plasma without WBCs was investigated.

Study design and methods: ATP- and ADP-induced platelet shape change, (shear-induced) aggregation and $\mathrm{Ca}^{2+}$ signaling were measured in the presence or absence of plasma. Degradation of nucleotides in plasma was quantified by high-performance liquid chromatography.

Results: Washed platelets became refractory for ATP and ADP in shape change, aggregation, and $\mathrm{Ca}^{2+}$ responses during a 90-minute incubation at $37^{\circ} \mathrm{C}$. The platelet responses mediated by $\mathrm{P} 2 \mathrm{X}_{1}, \mathrm{P} 2 \mathrm{Y}_{1}$, and $\mathrm{P} 2 \mathrm{Y}_{12}$ receptors gradually reduced or disappeared. When plasma was present, however, the platelets persistently showed high responses to ATP and ADP. Heat treatment of plasma abolished this effect. Also under conditions of flow and high shear, platelets in plasma kept high $\mathrm{P} 2 \mathrm{X}_{1}$ activity, mediating aggregate formation. In isolated plasma, not containing WBCs, nucleotides were degraded in the order of ADP/UDP > ATP/UTP. Degradation of ATP was partly inhibited by blocking the ecto-NTPDase CD39, whereas degradation of both ATP and ADP was inhibited by blocking ecto-pyrophosphatase/phosphodiesterase activity. Part of the nucleotide-degrading activities appeared to be membrane-bound.

Conclusion: Ecto-nucleotidases in plasma preserve the functionality of $\mathrm{P}_{2} \mathrm{X}_{1}$ and P2Y receptors. Upon platelet storage, these plasma activities are essential to ensure adequate (shear-dependent) formation of aggregates and thrombi. 


\section{Introduction}

The autocrine agents ADP and ATP, released from platelet-dense granules, play a key role in collagen-induced thrombus formation. ${ }^{90,91}$ Platelets contain two receptors for ADP, namely, $\mathrm{P}_{2} \mathrm{Y}_{1}$ and $\mathrm{P} 2 \mathrm{Y}_{12},{ }^{145,146}$ and one type of ATP receptor, the $\mathrm{P} 2 \mathrm{X}_{1}$ cation channel. ${ }^{147}$ The latter mediates a short-lived intracellular $\mathrm{Ca}^{2+}$ increase, which leads to shape change and contributes to platelet activation by other agonists. ${ }^{148-150}$ The $P 2 Y_{1}$ receptor signals via $G_{q \alpha}$ and causes phospholipase $\mathrm{C}$-mediated $\mathrm{Ca}^{2+}$ mobilization, shape change, thromboxane $A_{2}$ release, and initial aggregation. ${ }^{151,152}$ In contrast, the P2Y 12 receptor signals via $G_{i \alpha}$ and inhibition of adenylyl cyclase, which enhances $\mathrm{Ca}^{2+}$ mobilization and aggregation. ${ }^{153}$ In addition to $P 2 Y_{1}$, also $P 2 Y_{12}$ receptors have been proposed to play a role in reorganization of the actin cytoskeleton via RhoA/p160ROCK activation. ${ }^{154}$ Both $\mathrm{P} 2 \mathrm{Y}$ and $\mathrm{P} 2 \mathrm{X}_{1}$ types of platelet receptors are sensitive to desensitization due to autocrine production of ADP and ATP. In particular, $\mathrm{P}_{2} \mathrm{X}_{1}$ receptors are rapidly inactivated following ligand occupation. ${ }^{148,155}$ The desensitization mechanism of $\mathrm{P} 2 \mathrm{Y}_{1}$ and $\mathrm{P} 2 \mathrm{Y}_{12}$, at least in platelets treated with high concentrations of ADP, relies on phosphorylation by protein kinase $C$ and a G-protein-coupled receptor kinase, respectively. ${ }^{115}$ Once phosphorylated, the $\mathrm{P} 2 \mathrm{Y}_{1}$ receptors are internalized, whereas the $\mathrm{P} 2 \mathrm{Y}_{12}$ receptors tend to remain on the platelet surface. ${ }^{156}$ In vivo, this receptor desensitization is effectively prevented by membrane-bound ectonucleotidases, such as the ecto-NTPDase, CD39, expressed at the surface of endothelial cells and white blood cells (WBCs). These ATP- and ADP-degrading enzymes thus restrict the autocrine effects of activated platelets to sites of thrombus formation. ${ }^{157}$

The storage of platelets under blood bank conditions results in decreased platelet activation and aggregation responses toward ADP and other agonists. ${ }^{98}$ In this case, the inactivation is likely due to a slow release of adenine nucleotides, for example, leading to a diminished participation of stored platelets to the thrombus-forming process under conditions of shear. ${ }^{100}$ In this study, we investigated the hypothesis that storage affects the sensitivity of 
platelet purinergic receptors to ADP and ATP and, thereby, the quality of the platelets.

\section{Materials and methods}

\section{Materials}

Adenosine was from Roche (Basel, Switzerland). UMP, UDP, and UTP were from Boehringer (Mannheim, Germany); recombinant hirudin, from Pharmion (Hamburg, Germany); AR-C69931MX, from AstraZeneca (Mölndal, Sweden); DPhe-Pro-Arg chloromethyl ketone (PPACK), from Calbiochem (San Diego, CA); and anti-CD39 monoclonal antibody (MoAb) BU61 IgG1, from Ancell (Bayport, MI). Fura-2 and Oregon Green-488 (OG)-Bapta-1 acetoxymethyl esters were from Molecular Probes (Leiden, the Netherlands). Kits to measure vasodilatorstimulated phosphoprotein (VASP) phosphorylation were from Biocytex (Marseille, France); Horm-type collagen, from Nycomed (Munich, Germany); and Aggrastat (tirofiban), from MSD (Haarlem, the Netherlands). Control 12G1 MoAb (IgG1) was a gift from H. Deckmyn (Leuven, Belgium). Other reagents were from Sigma (St. Louis, MO).

\section{Blood cell preparation}

Whole blood was drawn from healthy volunteers after informed consent. Blood was collected in citrate (CPD, $1.9 \mathrm{mM}$ citric acid, $11.0 \mathrm{mM}$ trisodium citrate, $2 \mathrm{mM} \mathrm{NaH}_{2} \mathrm{PO}_{4}$, and $15.8 \mathrm{mM}$ glucose, $\mathrm{pH} 7.0$ ). In some cases, blood was collected in hirudin $(20 \mu \mathrm{g} / \mathrm{mL}$, f.c.). Citrate anti-coagulated blood was centrifuged at $240 \times g$ for 15 minutes, and platelet-rich plasma (PRP) was carefully removed, leaving the buffy coat untouched. Platelet-free plasma was prepared from fresh or stored PRP by centrifuging twice at $2650 \times g$ for 10 minutes. Microparticle-free plasma was obtained by centrifugation at $20,000 \times g$ for 20 minutes.

Platelet-plasma concentrates were prepared and stored under standard blood bank conditions. ${ }^{100}$ In brief, whole blood was spun down, and platelet-rich buffy coats from five donors were pooled with plasma from one donor. WBCs 
were removed by filtration. PRP, containing $1 \times 10^{12}$ platelets/L and fewer than $10^{6} \mathrm{WBCs} / \mathrm{L}$, was stored at $22^{\circ} \mathrm{C}$ in a flatbed shaker.

To prepare washed platelets, ${ }^{158}$ fresh or stored PRP was mixed with $1 / 15$ vol ACD solution ( $80 \mathrm{mM}$ trisodium citrate, $52 \mathrm{mM}$ citric acid, $180 \mathrm{mM}$ glucose) supplemented with $0.1 \mathrm{U} / \mathrm{mL}$ apyrase and then centrifuged at $800 \times g$ for 15 minutes. platelets were washed in the presence of apyrase, and finally resuspended in Hepes buffer, pH 7.45 (136 mM NaCl, $2.7 \mathrm{mM} \mathrm{KCl}, 10 \mathrm{mM}$ Hepes, $2 \mathrm{mM} \mathrm{MgCl}_{2}$, $2 \mathrm{mM}$ glucose, $1 \mathrm{mg} / \mathrm{mL}$ BSA) without apyrase. Cells were counted with a cell counter (Beckman Coulter, Miami, FL) and adjusted to $250 \times 10^{9}$ platelets/L. WBCs were isolated from buffy coats as described. ${ }^{159}$

\section{Platelet shape change and aggregation}

Platelet aggregation was determined under stirring conditions at $37^{\circ} \mathrm{C}$ with an optical aggregometer (Chrono-Log, Havertown, PA). Where indicated, citrate anti-coagulated PRP was recalcified in the presence of $10 \mu \mathrm{M}$ PPACK to avoid coagulation. Addition of $16.6 \mathrm{mM} \mathrm{CaCl}_{2}$ was required to achieve a physiological free $\mathrm{Ca}^{2+}$ concentration of 1.0 to $1.25 \mathrm{mM}^{160}$ Aggregation of washed platelets was measured in the presence of $1 \mathrm{mg} / \mathrm{mL}$ fibrinogen and $1 \mathrm{mM} \mathrm{CaCl}$. Platelet shape change was determined with the same equipment, where indicated in the presence of an allbß3 blocker, Aggrastat $(2 \mu \mathrm{g} / \mathrm{mL})$.

\section{VASP phosphorylation}

Phosphorylation of VASP was detected with a specific fluorescein-labeled MoAb after fixation of washed platelets, according to the instructions of the VASP phosphorylation kit (Biocytex). In platelets, VASP becomes phosphorylated at $\operatorname{Ser}^{157}$ by $C A M P$-elevating prostaglandin $E_{1}\left(P_{G} E_{1}\right)$; this phosphorylation is antagonized by $\mathrm{P}_{2} \mathrm{Y}_{12}$ activation. ${ }^{161}$ Thus, assay controls were carried out with platelets incubated with $\mathrm{PGE}_{1}$ and/or $\mathrm{ADP}$, according to the kit protocol. 


\section{Platelet adhesion under flow conditions}

Hirudin anti-coagulated PRP was perfused over a collagen-coated coverslip through a parallel-plate flow chamber at continuous wall-shear rate of 1000/s, as described for whole blood. ${ }^{90}$ MRS2159 $(100 \mu \mathrm{M})$ was used to block P2X ${ }_{1}$ mediated events. No cross-antagonism of MRS2159 with $\mathrm{P}_{2} \mathrm{Y}_{1-}$ or $\mathrm{P}^{2} \mathrm{Y}_{12^{-}}$ induced aggregation was seen at this concentration. ${ }^{162}$ During and after perfusion, high-resolution microscopic phase-contrast images were recorded in real-time with a digital imaging system (Visitech, Sunderland, UK). Surface area coverage with platelets was analyzed from at least 50 different collagencontaining microscopic fields.

\section{Cytosolic $\mathrm{Ca}^{2+}$ measurement}

Changes in cytosolic $\left[\mathrm{Ca}^{2+}\right]_{i}$ were monitored by ratiometric fluorescence measurements of washed, Fura-2-loaded platelets, with common calibration procedures. ${ }^{158}$ To measure $\left[\mathrm{Ca}^{2+}\right]_{i}$ in the presence of plasma, washed platelets were loaded with OG-Bapta-1 acetoxymethyl ester ( $8 \mu \mathrm{M}, 30 \mathrm{~min})$ and apyrase $(0.1 \mathrm{U} / \mathrm{mL})$. After centrifugation, the platelets were resuspended in autologous plasma. Fluorescence was recorded under stirring at $37^{\circ} \mathrm{C}$ in a spectrofluorometer (SLM-Aminco, Rochester, NY).

\section{Measurement of shear-induced platelet aggregation}

Shear-induced aggregation of anti-coagulated PRP was measured ${ }^{162}$ in a ring-shaped viscometer generating laminar flow (Ravenfield, Heywood, UK) at $37^{\circ} \mathrm{C}$ with $2 \mathrm{mM}$ free $\mathrm{Ca}^{2+}$. Shear rate was $9000 / \mathrm{s}$, corresponding to a shear stress of 80 dynes per $\mathrm{cm}^{2}$. At defined time points, samples were fixed in $1 \%$ paraformaldehyde; platelet aggregation was determined from reduction in single platelet count.

\section{Measurement of adenine and uracil nucleotides by high-performance} liquid chromatography

Plasma samples $(75 \mu \mathrm{L})$ were incubated with nucleotides at $37^{\circ} \mathrm{C}$. Where indicated, autologous WBCs were added at a final concentration of $5 \times 10^{9} / \mathrm{L}$. 
Reactions were stopped by deproteination with $20 \% \mathrm{HClO}_{4}(25 \mu \mathrm{L})$, and samples were centrifuged at $20,000 \times g$ for 10 minutes. Supernatants were neutralized with $400 \mu \mathrm{L}$ of $1 \mathrm{M}$ phosphate buffer and delipidated by extraction with $500 \mu \mathrm{L}$ of $50 \% n$-heptane. Resulting samples were used for highperformance liquid chromatography (HPLC) separation of nucleotides, as described. ${ }^{163}$ Aliquots of $20 \mu \mathrm{L}$ were injected into a $3-\mu \mathrm{m}$ Supelcosil LC18 column (Sigma). Nucleotides were separated with gradient elution of Buffer $A$ with methanol at a flow rate of $1 \mathrm{~mL}$ per minute: first, methanol was maintained at $0.5 \%$ for 9 minutes and then it was increased to $20 \%$ for 1 minute, followed by a plateau of $20 \%$ methanol during the next 4 minutes. Methanol was then decreased to $0.5 \%$ for 1 minute and kept at this level during the post-run period. Buffer A consisted of $6 \mathrm{mM}$ tetrabutylammonium phosphate in $0.1 \mathrm{M}$ phosphate buffer (pH 6.0). Nucleotides were monitored at $260 \mathrm{~nm}$ and quantified by integration of peak absorbance areas. Standard curves were constructed with purified nucleotides $(10-200 \mu \mathrm{M})$.

\section{Statistical analysis}

Data were compared with a paired (Wilcoxon) signed rank test. Data are presented as mean $\pm S D$, unless indicated otherwise.

\section{Results}

\section{Presence of plasma is required for persistent platelet aggregation and shape change induced by $\mathrm{P} 2 \mathrm{Y}$ and $\mathrm{P} 2 \mathrm{X}_{1}$ receptors}

Washed platelets were prepared from PRP that was either freshly isolated or stored for 4 to 5 days under blood bank conditions. Suspensions of these platelets were incubated at $37^{\circ} \mathrm{C}$ (without apyrase), and ADP- and ATP-induced responses were measured. Initially, washed platelets derived from fresh PRP had clear shape change and aggregation reactions with ADP $(10 \mu \mathrm{M})$. Incubation at $37^{\circ} \mathrm{C}$ for 90 minutes (without apyrase) resulted in complete loss of aggregation in response to a high dose of ADP, whereas the shape change persisted (figure 1A). Washed platelets derived from stored PRP had an 
incomplete aggregation response with ADP. Again, the aggregation disappeared after incubation at $37^{\circ} \mathrm{C}$, whereas the shape change remained. These results point to a gradual loss of ADP responsiveness.

Aggregation with ADP was also directly measured in fresh and stored PRP preparations. Before measurement, the PRP was recalcified in the presence of PPACK anti-coagulant to achieve physiological, millimolar concentrations of free $\mathrm{Ca}^{2+}$ and $\mathrm{Mg}^{2+}$. In both fresh and stored PRP, ADP $(10 \mu \mathrm{M})$ provoked marked shape change and aggregation responses (figure 1B). Typically, prolonged (90min) incubation at $37^{\circ} \mathrm{C}$ led to increased aggregation in stored PRP.

Both $\mathrm{P}_{2} \mathrm{Y}_{1}$ and $\mathrm{P} 2 \mathrm{Y}_{12}$ contribute to ADP-induced aggregation, whereas mainly $\mathrm{P}_{2} \mathrm{Y}_{1}$ is involved in shape change. Because aggregation of washed platelets but not shape change decreased in time, mainly the $P 2 Y_{12}$ pathway seemed to be desensitized. Epinephrine activates the $G_{i} / G_{z}$ pathway in a similar way to $P 2 Y_{12}$ ligandation. ${ }^{164}$ Therefore, washed platelets were incubated for 90 minutes at $37^{\circ} \mathrm{C}$ and co-stimulated with $30 \mu \mathrm{M}$ epinephrine and $10 \mu \mathrm{M}$ ADP. This led to a high aggregation reaction, indicating that epinephrine could bypass reduced $\mathrm{P}_{2} \mathrm{Y}_{12}$ function (figure 1C). To confirm this, platelets were stimulated with ADP in the presence of the selective $P 2 Y_{12}$ receptor antagonist, $20 \mu \mathrm{M}$ AR-C69931MX (currently known as cangrelor and used in clinical trials). ${ }^{165}$ This compound greatly reduced the aggregation response and left the shape change unaltered (figure 1C). Together, these results point to a reduction in P2Y ${ }_{12}$ activity in the absence of plasma, although cross-antagonism of yet to be identified receptors cannot be ruled out.

Further evidence for downregulation of $\mathrm{P}_{2} \mathrm{Y}_{12}$ activity came from measurements of $\mathrm{G}_{\mathrm{i}}$-mediated changes in VASP phosphorylation with a flowcytometric assay with fluorescent-labeled antiphospho-VASP MoAb. ${ }^{161}$ As required, cAMP elevation with $\mathrm{PGE}_{1}(25 \mathrm{nM})$ resulted in a high mean fluorescence intensity of platelets to $73.7 \pm 1.5$ (mean $\pm S E, n=3$ ). Simultaneous addition of ADP $(10 \mu \mathrm{M})$ to washed platelets decreased this level to $42 \pm 2.6$ after 5 minutes of incubation at $37^{\circ} \mathrm{C}$ and to $55.7 \pm 7.8$ after 90 minutes of incubation at $37^{\circ} \mathrm{C}$. This indicated decreased $G_{i}$ activity via $P 2 Y_{12}$ during the incubation period. 


\section{A washed platelets}
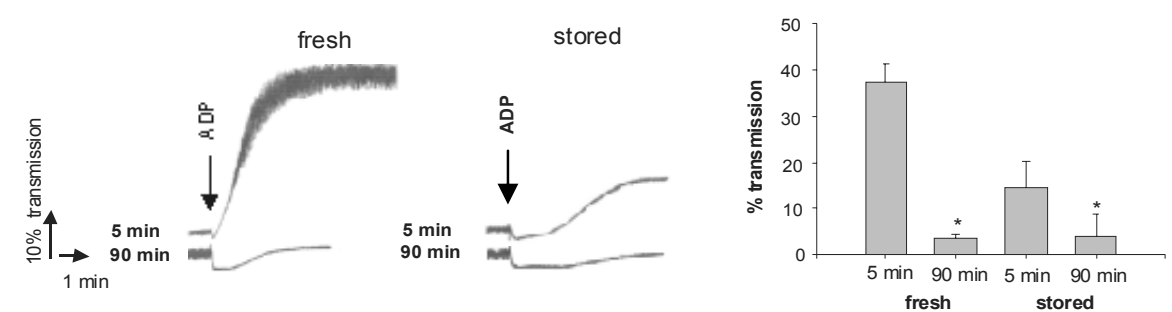

B PRP
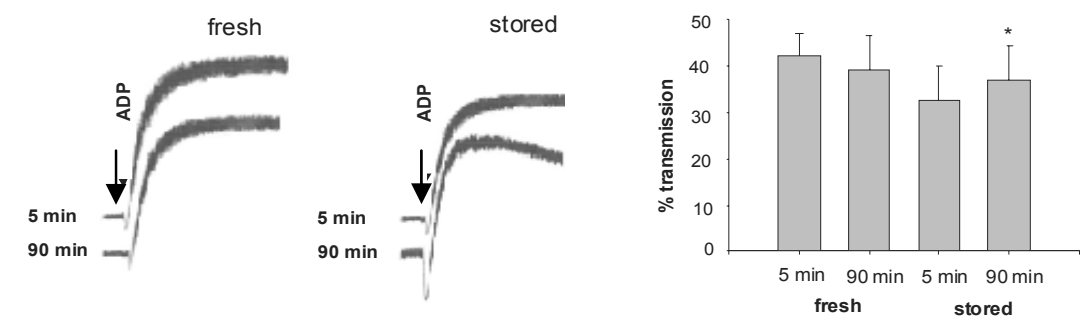

C washed platelets
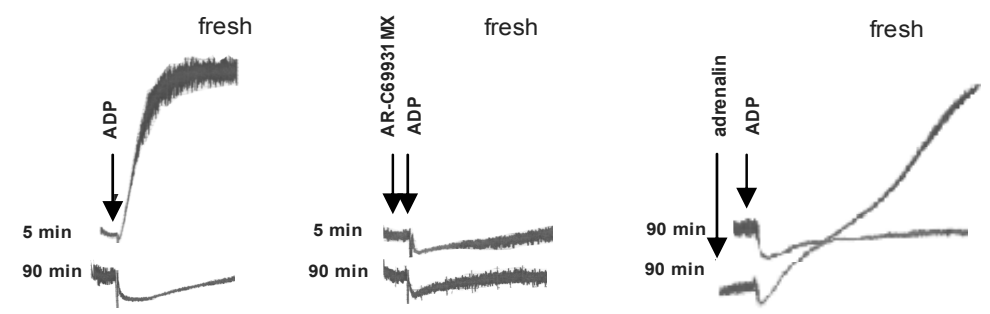

Figure 1. Reduced desensitization of ADP-induced aggregation in the presence of plasma Citrate anti-coagulated PRP was freshly isolated or stored for 4 to 5 days under blood bank conditions; it was in part used to prepare washed platelets. PRP and washed platelets $\left(250 \times 10^{9}\right.$ platelets/L) were incubated for 5 or 90 minutes $\left(37^{\circ} \mathrm{C}\right)$. (A) The washed platelets were stimulated with ADP $(10 \mu \mathrm{M})$ in the presence of fibrinogen $(1 \mathrm{mg} / \mathrm{mL})$ and $\mathrm{CaCl}_{2}(1 \mathrm{mM})$. (B) The PRP was recalcified with $16.6 \mathrm{mM} \mathrm{CaCl}_{2}$ in the presence of $10 \mu \mathrm{M}$ PPACK and stimulated with $10 \mu \mathrm{M}$ ADP (C) Freshly isolated, washed platelets were treated with vehicle (left), $20 \mu \mathrm{M}$ AR-C69931MX (middle), or $30 \mu \mathrm{M}$ epinephrine (right) and stimulated with $10 \mu \mathrm{M}$ ADP. Shown are representative traces and bar histograms of maximal changes in transmission from five independent experiments, with blood or PRP from different donors (mean \pm SD). ${ }^{*} p<0.01$ compared to 5 minutes. 

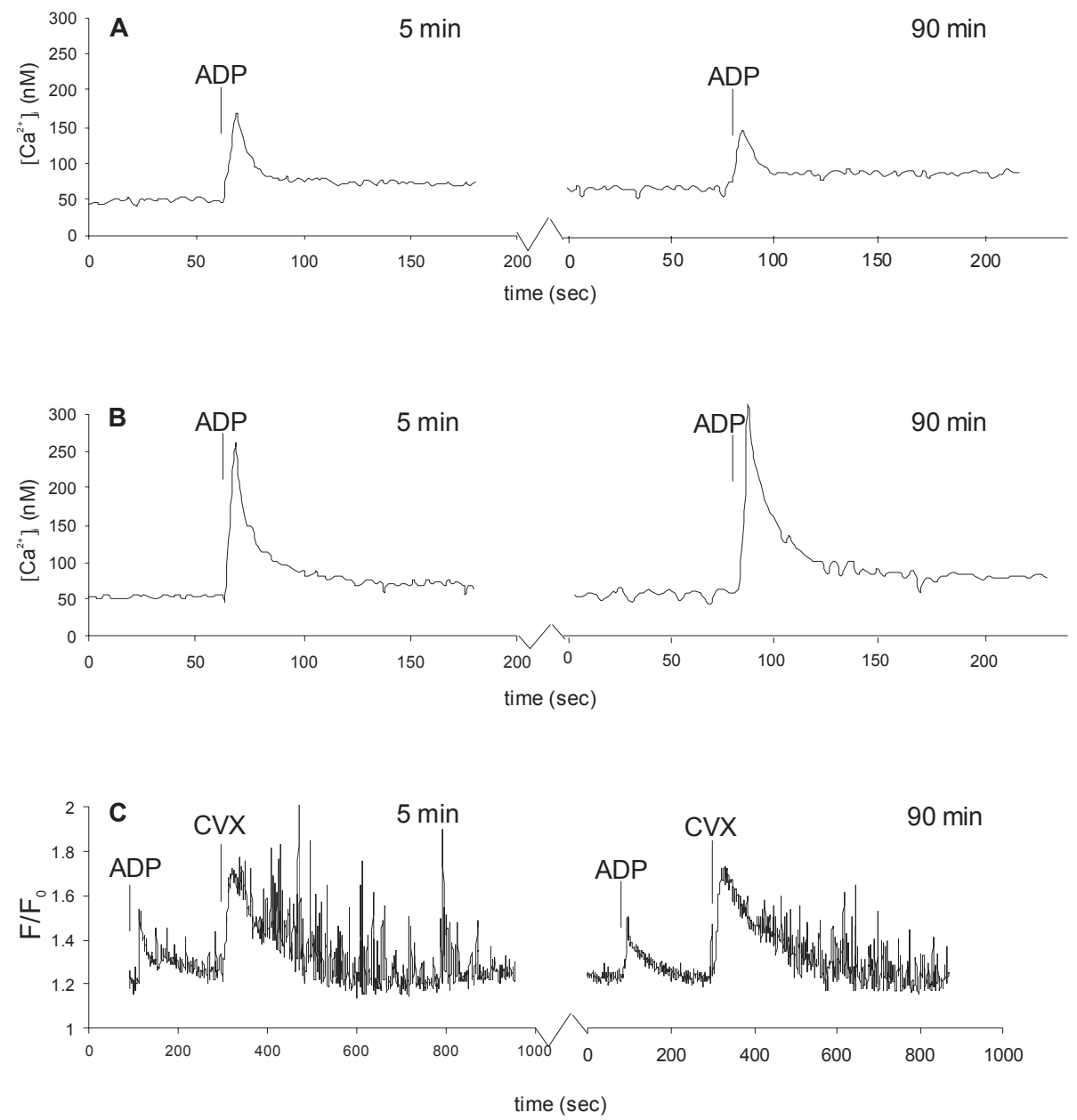

Figure 2. Presence of apyrase or plasma prevents desensitization of ADP-induced $\mathrm{Ca}^{2+}$ mobilization. (A and B) Washed, Fura-2-loaded platelets were incubated at $37^{\circ} \mathrm{C}$ for 5 to 90 minutes; increases in $\left[\mathrm{Ca}^{2+}\right]_{\text {i }}$ were measured upon stimulation with ADP $(20 \mu \mathrm{M})$. Platelets were untreated (A) or treated with $0.05 \mathrm{U} / \mathrm{mL}$ apyrase (B). (C) Platelets loaded with OG-Bapta-1 were added to plasma and incubated at $37^{\circ} \mathrm{C}$ for 5 to 60 minutes. Changes in $\left[\mathrm{Ca}^{2+}\right]_{i}$ were measured upon stimulation with ADP $(20 \mu \mathrm{M})$ and convulxin $(\mathrm{CVX}, 20 \mathrm{ng} / \mathrm{mL})$. Representative, semi-calibrated traces of $F / F_{0}$ are given 
Changes in $\mathrm{G}_{\mathrm{q}}$-mediated $\mathrm{P} 2 \mathrm{Y}_{1}$ activity (with minor contribution of $\mathrm{P} 2 \mathrm{Y}_{12}$ ) were obtained from $\mathrm{Ca}^{2+}$ measurements in platelets loaded with fluorescent $\mathrm{Ca}^{2+}$-sensitive probes. ${ }^{149}$ In washed, Fura2-loaded platelets, a 90-minute treatment at $37^{\circ} \mathrm{C}$ resulted in a $43 \pm 3.6 \%$ decrease in ADP-induced $\mathrm{Ca}^{2+}$ response (figure $2 \mathrm{~A}$ ). This decrease was completely antagonized by treatment of the platelets with apyrase (figure 2B). No such decrease was observed in OG-Bapta-1-loaded platelets that were incubated for 90 minutes in the presence of plasma (figure $2 \mathrm{C}$ ). This points to diminished $\mathrm{P}_{2} \mathrm{Y}_{1}$-mediated $\mathrm{Ca}^{2+}$ signal, likely due to autocrine ADP secretion, only in washed platelets.

To investigate $\mathrm{P} 2 \mathrm{X}_{1}$ activity, platelets were stimulated with the specific agonist, $\alpha, \beta M e A T P(2 \mu \mathrm{M})$, in the presence of $1 \mathrm{mM} \mathrm{CaCl} \mathrm{Cl}_{2}$ to allow $\mathrm{Ca}^{2+}$ influx. When added to washed platelets (apyrase $[0.2 \mathrm{U} / \mathrm{mL}$ ] was present during centrifugation, but not in the resuspension buffer), $\alpha, \beta M e A T P$ failed to induce shape change (not shown), which confirmed the rapid disappearance of this receptor activity by previous centrifugation. ${ }^{148}$ Similarly, $\alpha, \beta$ MeATP did not induce shape change in freshly isolated PRP, but this response reappeared after prolonged incubation at $37^{\circ} \mathrm{C}$ (figure $3 \mathrm{~A}$ ). Surprisingly, $\alpha, \beta M e A T P$ evoked a clear platelet shape change in stored PRP, which further increased upon $37^{\circ} \mathrm{C}$ incubation. Incubation at $37^{\circ} \mathrm{C}$ also led to recovery in shape change evoked by the physiological $\mathrm{P} 2 \mathrm{X}_{1}$ agonist ATP (figure $3 \mathrm{~B}$ ). Specificity of the activity of $\alpha, \beta M e A T P$ was confirmed by the finding that its effects were fully inhibited with the P2X 1 blocker MRS2159 $(300 \mu \mathrm{M})$. From these data, we conclude that downregulation of platelet activation by $\alpha, \beta M e A T P$ and ATP via $P 2 X_{1}$ can be prevented by suspending platelets in plasma preferably at $37^{\circ} \mathrm{C}$.

During the 90-minute incubation, the ATP-induced shape change increased from 0 to $12.5 \pm 4.4 \%$ transmission (figure $3 \mathrm{~B}$ ). Because this response may contain a $\mathrm{P} 2 \mathrm{Y}_{1}$ component due to contaminating and/or degraded ATP, we also measured ADP-induced shape change in the presence of allbB3 inhibitor, Aggrastat. Indeed, shape change with ADP slightly increased with $35.5 \pm 0.2 \%$ $(n=4)$ by incubation at $37^{\circ} \mathrm{C}$. These results thus indicate that initially only the $\mathrm{P} 2 \mathrm{X}_{1}$ component is absent (which is provoked by $\alpha, \beta M e A T P$ ); incubation at $37^{\circ} \mathrm{C}$ then increases both the $\mathrm{P} 2 \mathrm{X}_{1}$ and the $\mathrm{P} 2 \mathrm{Y}_{1}$ components. 


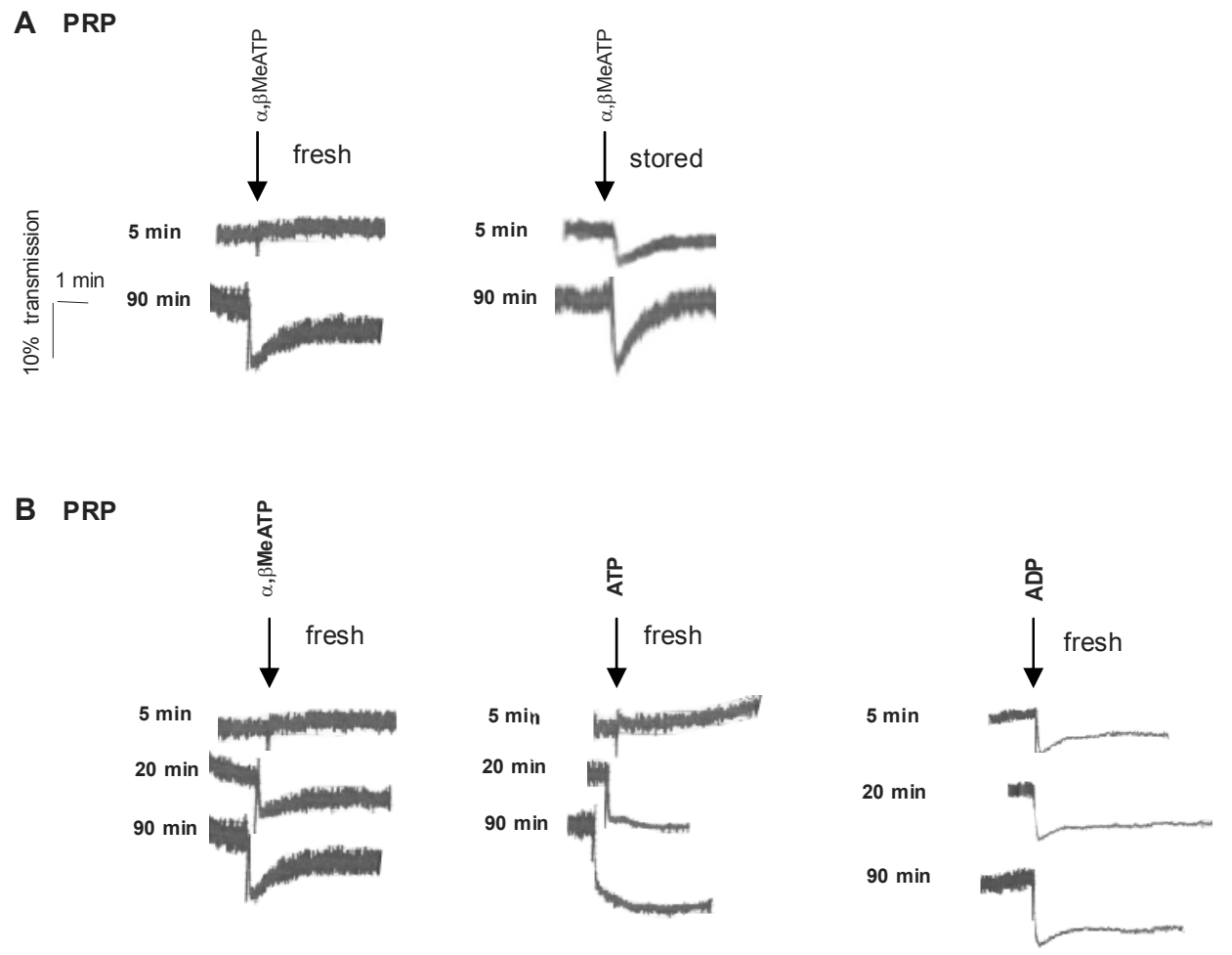

Figure 3. Plasma incubation recovers $\mathbf{P} 2 \mathbf{X}_{1}$ receptor desensitization. PRP was freshly isolated or stored for 4 to 5 days under blood bank conditions $\left(250 \times 10^{9}\right.$ platelets/L), incubated at $37^{\circ} \mathrm{C}$ for 5 to 90 minutes, and recalcified in the presence of PPACK. (A) Shape change was measured with $2 \mu \mathrm{M} \alpha, \beta M e A T P$. (B) Shape change in fresh PRP was measured in response to $2 \mu \mathrm{M} \alpha, \beta M e A T P$, $5 \mu \mathrm{M}$ ATP, or $10 \mu \mathrm{M}$ ADP plus $2 \mu \mathrm{g} / \mathrm{mL}$ Aggrastat. Representative traces are given $(\mathrm{n}=3)$.

\section{Heat treatment of plasma abolishes recovery of P2Y-mediated platelet responses}

To determine whether an enzymatic component contributes to the preserving effect of plasma on platelet activation, samples were subjected to hightemperature treatment $\left(1 \mathrm{~h}\right.$ at $\left.60^{\circ} \mathrm{C}\right)$. The platelets were then resuspended in either heat-treated or untreated (control) plasma. Only platelets in heat-treated plasma did no longer aggregate with ADP, but still underwent shape change (figure 4B). This loss in aggregation was typically prevented by addition of apyrase. In the latter experiment, the cells were stimulated with the stable ADP analog, 2MeSADP $(5 \mu \mathrm{M})$, to avoid breakdown of the agonist by apyrase (figure 

A control
B heat-treated
C heat-treated + apyrase
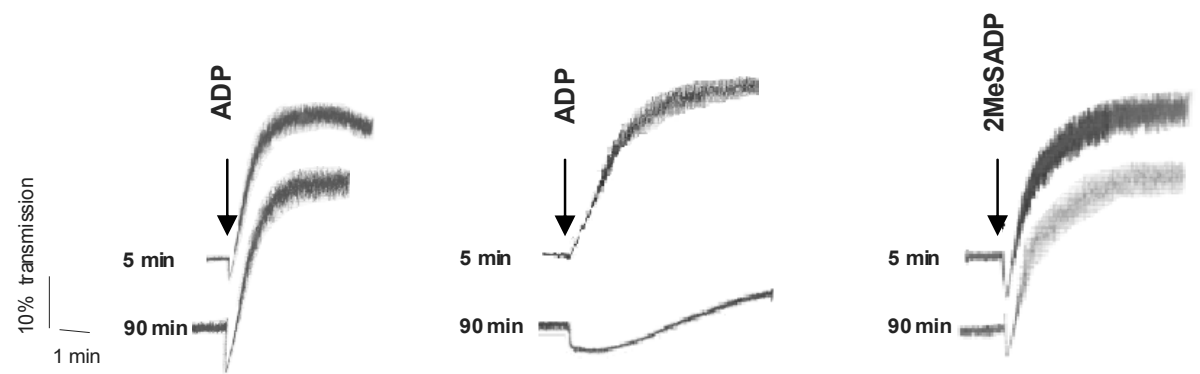

Figure 4. Desensitization of $\mathrm{P} 2 \mathrm{Y}$-induced aggregation in heat-treated plasma. Hirudin anticoagulated plasma was heat-treated at $60^{\circ} \mathrm{C}$ for 1 hour or remained untreated. Platelets were suspended in (A) control plasma, $(B)$ heat-treated plasma, or $(C)$ heat-treated plasma plus apyrase $\left(0.1 \mathrm{U}\right.$ ADPase $/ \mathrm{mL}$ ). After incubation at $37^{\circ} \mathrm{C}$ for 5 to 90 minutes, platelets were stimulated with $10 \mu \mathrm{M}$ ADP (A and B) or $5 \mu \mathrm{M} 2 \operatorname{MeSADP}(C)$. Shown are representative traces $(n=3)$. Similar results were obtained with heat-treated, citrate anti-coagulated plasma.

4C). These results suggest that a heat-sensitive component in plasma prevents ADP receptor desensitization.

Preserved $\mathrm{P}_{2} \mathrm{X}_{1}$ receptor activity of platelets in plasma play a key role in aggregate formation at high shear stress

We studied the functional importance of the preserved $\mathrm{P}_{2} \mathrm{X}_{1}$ activity in flow studies, knowing that $\mathrm{P} 2 \mathrm{X}_{1}$, via ATP secretion, potentiates collagen-induced thrombus formation under flow ${ }^{18}$ and enhances shear-induced platelet aggregation. ${ }^{150}$ Hirudin anti-coagulated PRP (without apyrase) was first incubated at $37^{\circ} \mathrm{C}$ to allow full $\mathrm{P} 2 \mathrm{X}_{1}$ resensitization and then perfused over collagen at a shear rate of $1000 /$ s. The resulting platelet adhesion and aggregate formation was significantly reduced by prior treatment of the platelets with $\alpha, \beta$ MeATP $(2 \mu \mathrm{M})$, to down regulate $P 2 X_{1}$ receptors (figure 5). Incubation with the specific P2X ${ }_{1}$ blocker MRS2159 $(100 \mu \mathrm{M})$ gave a similar effect. At this concentration, MRS2159 had no effect on P2Y receptors, as confirmed by aggregation experiments. Furthermore, measurements of shear-induced platelet aggregation, involving $\mathrm{P} 2 \mathrm{X}_{1}$ activity, pointed to an increased aggregation from $7.98 \pm 10$ to $27.7 \pm 9.6 \%(n=4, p=0.05)$ by incubation of fresh PRP at $37^{\circ} \mathrm{C}$ for 90 minutes (not shown). Together, these results suggest that recovered $\mathrm{P} 2 \mathrm{X}_{1}$ activity, achieved by platelet incubation in plasma, enhances the aggregate formation under shear conditions. 
A

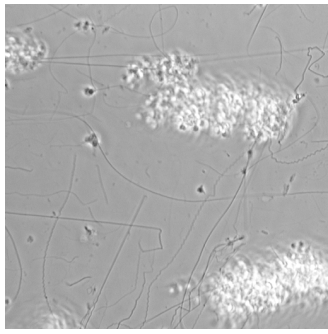

PRP

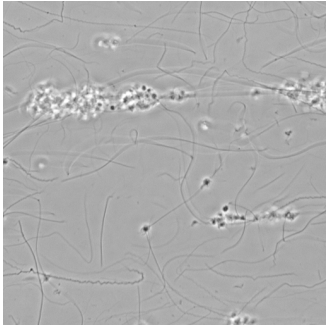

$\alpha, \beta \mathrm{MeATP}$

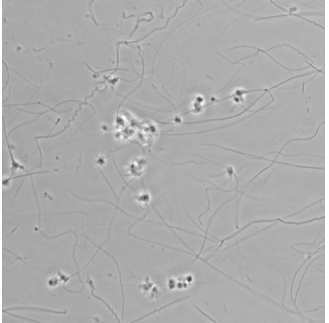

MRS2159

B

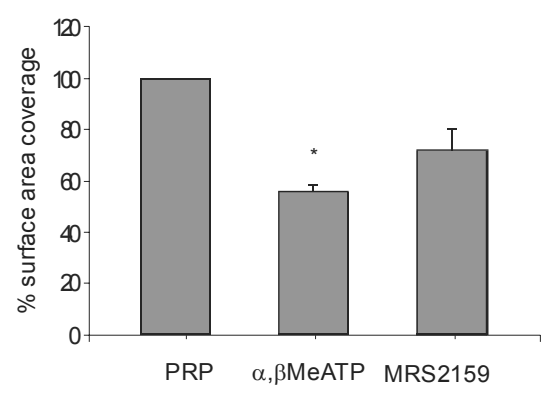

Figure 5. Preserved $P 2 X_{1}$ receptor function of platelets in plasma contributes to thrombus formation at high shear. Fresh PRP, hirudin-anti-coagulated and incubated for 30 minutes at $37^{\circ} \mathrm{C}$, was left untreated (control) or incubated with P2X ${ }_{1}$ antagonist $\alpha, \beta M e A T P(2 \mu \mathrm{M})$ or MRS2159 $(100 \mu \mathrm{M})$ before perfusion over collagen at shear rate of $1000 / \mathrm{s}$. (A) Representative images of thrombi are given after 4.5 minutes of perfusion. (B) Averaged surface area coverage with platelets in comparison to control condition $(1.87 \pm 1.22 \%)$. Data are means \pm SEM $(n=7)$. ${ }^{*} p<0.05$ for $\alpha, \beta M e A T P$ and $p=0.12$ for MRS2159, compared to control condition.

Plasma contains various enzymatic activities degrading both ATP and ADP

HPLC analysis was performed to investigate the possible enzymatic degradation of adenine nucleotides in plasma. In freshly isolated plasma, free from platelets and WBCs, both ATP and UTP were efficiently degraded by incubation at $37^{\circ} \mathrm{C}$ (figure $6 \mathrm{~A}$ ). Products formed were $A D P+A M P$ and UDP+UMP, respectively (table 1 ). Conversion of ATP was not influenced by the presence or absence of millimolar $\mathrm{Ca}^{2+}$, and it was similar in freshly isolated and stored plasmas. In addition, plasma degraded ADP and UDP at a three times higher rate in comparison to the trisphosphate analogs (figure 6B). Plasma preparations derived from four donors differed less than two-fold in these activities. 
Table 1. Overview of enzymatic conversion of adenines and uridines in plasma

\begin{tabular}{llcr} 
Substrate & \multicolumn{3}{c}{ Products } \\
\cline { 2 - 4 } NTP & NTP & NDP & NMP \\
\hline ATP & NA & + & + \\
ADP & Trace & NA & + \\
UTP & NA & - & + \\
UDP & - & NA & + \\
\hline NA $=$ not applicable & &
\end{tabular}

NA $=$ not applicable

We next investigated the possible contribution of ecto-NTPDase CD39 to the nucleotide degrading activity. In plasma treated with a saturating dose of antiCD39 MoAb, BU61 (30 $\mu \mathrm{g} / \mathrm{mL})$, we found a strong reduction of ATP-degrading activity, whereas $A D P$ degradation was left unchanged (figures $6 A$ and $6 B$ ). Treatment with an isotype IgG1 control (MoAb 12G1) was without effect $(2.7 \%$ of ATP converted), confirming the specificity of the BU61 MoAb. In other samples, plasma was treated with Ap5A $(100 \mu \mathrm{M})$, which inhibits ectonucleotide pyrophosphatase-phosphodiesterase (ecto-NPPase) activity. With Ap5A present, both the ATP- and the ADP-degrading activities reduced by $50 \%$. Furthermore, the combination of BU61 and Ap5A blocked most of the ATP and ADP conversion (figure 6). Other control experiments indicated that the degradation of ATP and ADP was blocked by prior heat treatment of plasma with 80 and $95 \%$, respectively, and that it increased by 2.0 - and 3.1 -fold after addition of WBCs $\left(5 \times 10^{9} / \mathrm{L}\right)$ to untreated plasma.

Because ecto-NTPDases and ecto-NPPases are membrane-bound components of WBCs and endothelial cells, ${ }^{166}$ we considered the possibility that membrane-containing remnants or microparticles derived from these cells were responsible for the nucleotide degradation. To investigate this, ATP and ADP conversion was measured in plasma samples that were centrifuged at high speed to remove all particulate material. In the supernatants, ATP degradation was almost abolished, whereas ADP-degrading activity was about halved (figure 6C). Further, addition of platelets $\left(250 \times 10^{9} / \mathrm{L}\right)$ to plasma did not influence the conversion of ATP and ADP. Together, this indicates that at least two different enzymatic activities, partly present in particulate, membrane-bound form, are responsible for the adenine nucleotide-degrading activity in plasma (figure 6D). 
A

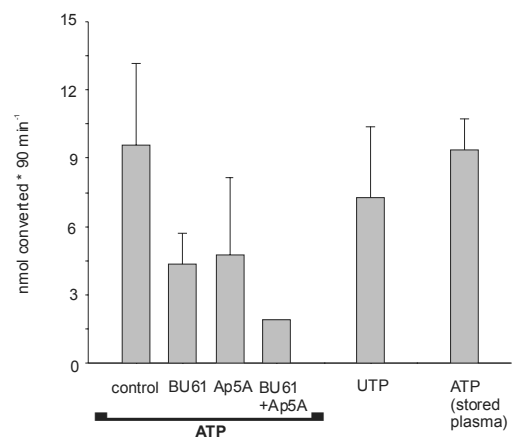

c

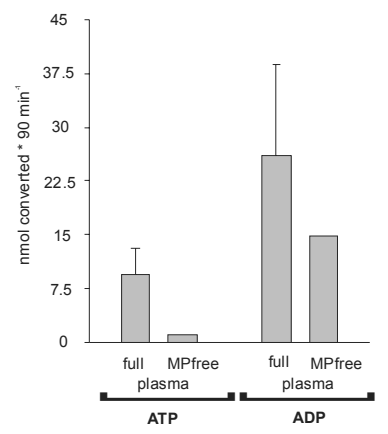

B

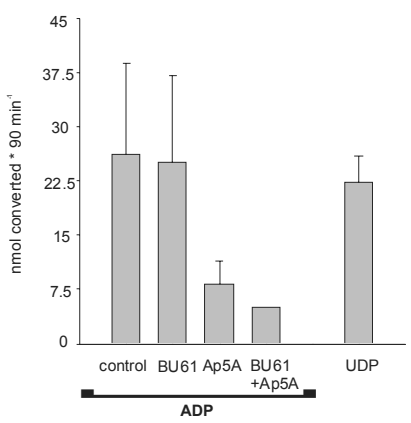

D

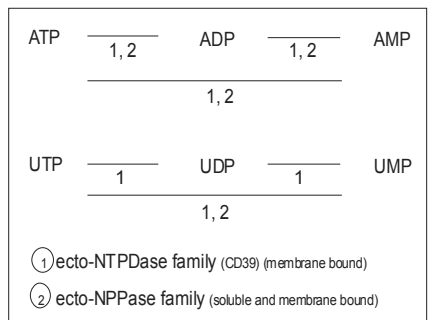

Figure 6. Degradation of adenosine and uracil nucleotides in plasma. (A) Human plasma $(75 \mu \mathrm{L})$ was either freshly isolated or stored for 4 to 5 days and incubated with ATP or UTP (1 mM) for 0 to 90 minutes at $37^{\circ} \mathrm{C}$. Vehicle buffer, anti-CD39 MoAb BU61 $(30 \mu \mathrm{g} / \mathrm{mL})$, and/or Ap5A $(100 \mu \mathrm{M})$ were added. (B) Freshly isolated plasma $(75 \mu \mathrm{L})$ was incubated with ADP or UDP (each $1 \mathrm{mM}$ ) for 0 to 90 minutes at $37^{\circ} \mathrm{C}$. (C) Freshly isolated plasma was untreated (control) or centrifuged at $20,000 \times g$ for 20 minutes to obtain microparticle (MP)-free plasma. Incubation was with ATP or ADP $(1 \mathrm{mM})$ for 0 to 90 minutes at $37^{\circ} \mathrm{C}$. Nucleotide concentrations were determined by HPLC. Plasma samples from different donors were used (mean $\pm S D, n=2-3$ ). (D) Overview of nucleotidase activity in plasma according to HPLC analysis.

\section{Discussion}

Platelet responses to ADP and ATP are considered to be unstable and highly sensitive to purinergic receptor desensitization. In this article, we demonstrated that blood plasma prevents this desensitization, because it contains enzymatic activities that degrade ADP and ATP. Plasma thus preserves the functions of $P 2 Y_{1}$ and $P 2 Y_{12}$ and, surprisingly, also that of $P 2 X_{1}$ receptors. Particularly the latter is known to disappear within tens of minutes after platelet isolation. ${ }^{167}$ We noticed that even platelets that were prolongedly stored in plasma showed full $\mathrm{P} 2 \mathrm{X}_{1}$ activity (ATP-induced shape change). This is 
of physiological importance, because the retained $\mathrm{P} 2 \mathrm{X}_{1}$ function can play an important role in shear-induced thrombus formation.

The refractoriness of stored, plasma-free platelets toward ADP is likely to involve both $\mathrm{P} 2 \mathrm{Y}$ receptors. In such platelets, signaling of $\mathrm{P} 2 \mathrm{Y}_{12}$ is affected, as is concluded from a reduction in AR-C69931MX-inhibitable aggregation and a lower $\mathrm{G}_{\mathrm{i}}$-mediated VASP phosphorylation. Evidence for reduced $\mathrm{P}_{2} \mathrm{Y}_{1}$ action comes from the gradual decrease in ADP-induced $\mathrm{Ca}^{2+}$ responses. In contrast, such 'desensitized' platelets displayed a normal shape change with ADP, which suggests that incomplete (submaximal) $\mathrm{Ca}^{2+}$ signaling is sufficient for full shape change, most likely via the myosin light-chain kinase pathway. Part of the ADPinduced shape change can also be mediated through thromboxane and/or $\mathrm{Ca}^{2+}$ independent p160ROCK signaling via $\mathrm{G}_{12 / 13}$, as demonstrated by control experiments with the p160ROCK inhibitor Y-27632 in the presence of a $\mathrm{Ca}^{2+}$ chelator DM-BAPTA (data not shown). Importantly, we also found that plasma addition to washed platelets caused resensitization of either P2Y receptor type, indicating that this receptor desensitization (by weak autocrine activation) is fully reversible and is unlikely due to receptor cleavage and internalization.

Recent evidence indicates that $\mathrm{P} 2 \mathrm{X}_{1}$, in spite of its rapid desensitization, plays a crucial role in the regulation of thrombus formation in vivo and in vitro. ${ }^{162,168}$ In flow experiments, carried out at a moderately high shear rate of $1000 / s$, we indeed found that $P 2 X_{1}$ activity of platelets stored in plasma significantly contributes to the formation of thrombi on collagen. This conclusion was confirmed by shear-induced platelet aggregation experiments, which indicated that restoration of the purinergic receptor activity of platelets in plasma was accompanied by increased aggregate formation under shear.

A substantial part of the ATP-degrading activity in cell-free plasma was inhibited by treatment with an anti-CD39 MoAb while, in contrast, the ADPdegrading activity was not influenced by this MoAb. CD39 is a membranebound enzyme, also designated as ecto-NTPDase1, which in mice plays an important inhibitory role in the thrombotic process in vivo. ${ }^{157,169}$ Ultraspeed centrifugation of plasma resulted in complete disappearance of the ATP degradation, from which we conclude that this activity is particulate or 
membrane-bound, probably derived from blood or vascular cells. Accordingly, normal plasma contains particulate CD39 activity, which can preserve the fragile platelet $P 2 X_{1}$ activity.

The same experiments indicated that CD39 does not account for the degradation of ADP (UDP) into AMP (UMP) in cell-free plasma, which activity was typically higher than that of ATP degradation. The inhibition with Ap5A, which halved the conversion of ATP and ADP, points to involvement of ectoNPPases. Like CD39, ecto-NPPases are shed from the plasma membrane of endothelial cells and WBCs and then appear in plasma as membrane-bound or soluble forms. ${ }^{166}$ We conclude that this activity is of key importance in the prevention of $\mathrm{P} 2 \mathrm{Y}$ receptor desensitization. Because plasma samples prepared from stored PRP, which are greatly enriched in platelet-derived membrane fragments, ${ }^{100}$ have ATP and ADP conversion rates similar to that of freshly isolated, microparticle-free plasma, it is unlikely that platelet membranes contribute to the nucleotide degradation. This agrees with published evidence that CD39 is barely detectable in platelets. ${ }^{170}$ Confirming experiments showed that WBCs added to plasma, indeed, significantly increased the degradation of both ATP and ADP.

In summary, we conclude that non-cellular ATP- and ADP-degrading activities in plasma preserve the purinergic receptor activities of stored platelets. This is of physiological importance, because such nucleotide degradation in plasma allows platelets to recover from desensitization under conditions where they do not adhere to vascular endothelium or WBCs. The finding that platelets stored in plasma retain all their purinergic receptor functions is also of immediate practical relevance. At blood banks, there is a current tendency to store platelets for use in transfusion in synthetic media with reduced plasma contents. Platelets stored in such media may thus have a reduced responsiveness to ADP and ATP.

Acknowledgment: We thank C. Oury, PhD (Liège, Belgium); J. Sévigny, PhD (Québec, Canada); and J.Keuren, PhD (Maastricht, the Netherlands), for valuable advice. We also thank M.J. Drittij for technical HPLC analysis. 


\title{
Chapter 4
}

\section{Platelet ADP response deteriorates in synthetic storage media}

\author{
Jeffrey F.W. Keuren, Sandra Cauwenberghs, \\ Judith Heeremans, Wim de Kort, \\ Johan W.M. Heemskerk and Joyce Curvers \\ Transfusion \\ 2006; 46:204-12
}

Reprinted with permission 


\section{Abstract}

Background: During storage under blood bank conditions, platelets are known to secrete ADP. Platelet stimulation by $A D P$ results in refractoriness to restimulation, making this response one of the most unstable platelet reactions. The goal of this study was to evaluate the ADP-induced responses of platelets stored in full plasma or in plasma and additive solution (AS).

Study design and methods: Surface expression of P-selectin, ADP-induced aggregation, and reconstituted whole-blood thrombus formation were determined on collagen surfaces in a perfusion model with platelets that were stored for 4 days either in plasma or in the presence of plasma with PAS-II or Composol.

Results: After 4 days of storage in PAS-II but not in Composol, the percentage of platelets that had secreted granule contents (P-selectin) was increased, when compared to platelets stored in full plasma. Maximal aggregation in response to ADP was reduced for platelets stored in PAS-II or Composol. Resuspension of these platelets in plasma at $37^{\circ} \mathrm{C}$ for 1 hour caused partial recovery of the aggregation response. Addition of apyrase to platelets in AS preserved the responsiveness toward ADP. Titration experiments indicated that this response gradually decreased with decreasing plasma concentration. The functional significance of these findings was demonstrated by perfusion experiments. Thrombus formation on collagen was significantly higher for platelets stored in full plasma than for platelets stored in PAS-II or Composol.

Conclusions: Storage of platelets in the presence of AS under blood bank conditions induces deterioration of the platelet responsiveness to ADP compared to platelet concentrates in $100 \%$ plasma. Higher plasma-to-AS ratios result in better preserved responses. 
The present tendency to store platelets in synthetic media (additive solution [AS]) instead of full plasma has several apparent advantages. The replacement of plasma by an AS reduces the levels of antibodies in the platelet product, resulting in a reduction of the number of transfusion reactions. ${ }^{76,171}$ It further increases the efficiency of most pathogen inactivation systems. ${ }^{172}$ In addition, partial replacement of plasma by AS saves the plasma for other purposes, such as transfusion or fractionation. A number of studies have assessed the quality of platelets stored in such synthetic media and concluded that the quality of platelets was not affected. ${ }^{173-175}$ It was further demonstrated that addition of magnesium and potassium to $\mathrm{AS}$ improves $\mathrm{pH}$ and hypotonic shock response of stored platelets and reduces the concentrations of platelet-derived cytokines during storage. ${ }^{176,177}$ With regard to platelet functionality, one clinical study has shown that 24-hour platelet count increments were not significantly different in patients receiving platelets that were stored in either plasma or PAS-II, suggesting that transfusion with each preparation gives a similar hemostatic improvement. ${ }^{178}$ In contrast, two recent studies have shown that the deprivation of platelets from plasma can accelerate a loss of function, such as decreased aggregation with $A D P$, and induce constitutive death of platelets and subsequent scavenger receptor-mediated phagocytosis. ${ }^{111,179}$ In addition, the function of platelets stored in AS may be changed, because P-selectin expression was found to increase on platelets stored for 5 days in PAS-II compared with storage in full plasma. ${ }^{180,181}$ The objective of this study was to determine the functionality, and in particular ADP responsiveness, of platelets stored in full plasma compared to platelets stored in AS in the presence of $30 \%$ plasma.

ADP plays a key role in platelet activation. Once secreted from the dense granules, ADP amplifies platelet responses induced by numerous agonists, including collagen and thromboxane. ${ }^{151}$ The clinical significance of this amplifying effect of ADP may appear from the observation that patients with deficiencies in storage of ADP, granule secretion, or ADP receptor function can develop bleeding diatheses. ${ }^{151}$ In addition, drugs such as clopidogrel that antagonize ADP receptor function have a platelet-inhibiting effect in vivo 
(reviewed in Kunapuli and colleagues ${ }^{152}$ and Cattaneo and Gachet ${ }^{182}$ ). After activation by ADP, platelets become refractory to restimulation, likely due to desensitization of the ADP receptors. ${ }^{115,156}$

During storage under blood bank conditions, platelets continuously release granular ADP. ${ }^{127}$ Such autocrine release of ADP can lead to rapid desensitization of ADP responses. At present, it is unclear whether storage of platelets in a synthetic storage medium influences their functionality.

This study evaluated the responsiveness, in particular toward ADP, of platelets stored in full plasma or under conditions where the plasma was partly replaced by synthetic solutions, that is, PAS-II or Composol, the latter AS containing magnesium and potassium. As a marker of granule release, we determined surface expression of P-selectin, and we measured platelet ADP responses by aggregometry. To study ADP effects under more physiologically relevant conditions, we reconstituted stored platelets with plasma and red blood cells (RBCs) and perfused these at an arterial shear rate over a collagen surface. Finally, we determined which plasma content could preserve ADPinduced activation responses during storage.

\section{Materials and methods}

\section{Materials}

Fluorescein isothiocyanate (FITC)-labeled monoclonal antibody (MoAb) against $\mathrm{P}$-selectin was from Immunotech (clone thromb-6 supplied by Beckman Coulter, Mijdrecht, the Netherlands). FITC-labeled $\lg _{1}$ was from BectonDickinson (Aalst, Belgium). Apyrase (enzyme from potato that converts ADP to AMP plus $\mathrm{P}_{\mathrm{i}}$ ), ADP, 2-methyl-thio-ADP (2MeSADP), thromboxane $\mathrm{A}_{2}$ analog U46619, and bovine serum albumin (BSA) were from Sigma (St. Louis, MO). The selective irreversible active-site inhibitor of thrombin D-Phe-Pro-Arg chloromethyl ketone (PPACK) was from Calbiochem (San Diego, CA). Hormtype fibrillar collagen I was obtained from Nycomed (Munich, Germany). Arachidonic acid came from Kordia (Leiden, the Netherlands). Thrombin receptor-activating peptide (TRAP) is a hexapeptide (SFLLRN) that specifically 
activates protease-activated receptor $1^{183}$ and was purchased from Bachem (Bubendorf, Switzerland).

\section{Preparation of pooled platelets in plasma or AS}

Pooled platelet products were prepared as described previously. ${ }^{100}$ In short, whole blood was collected in citrate phosphate dextrose (CPD) with standard sterile procedures. Blood was left overnight on isobutenal cooling plates and thereafter centrifuged at $3700 \times g$ for 13 minutes. Plasma and RBCs were removed with a separation device (Compomat G4, Fresenius Hemocare, Friedberg, Germany, for plasma pools or Optipress, Baxter, Deerfield, IL, for the pools in AS), and buffy coats were left undisturbed for at least 2 hours. Buffy coats from five ABO- and Rhesus-identical donors were pooled with plasma from one donor, 70\% PAS-II (116 mM NaCl, $10 \mathrm{mM} \mathrm{Na}{ }_{3}$-citrate, $30 \mathrm{mM} \mathrm{Na}$ acetate, $\mathrm{pH} 7.2$; Baxter) and $30 \%$ plasma or $70 \%$ Composol $(90 \mathrm{mM} \mathrm{NaCl}$, $5 \mathrm{mM} \mathrm{KCl}, 1.5 \mathrm{mM} \mathrm{MgCl}_{2}, 11 \mathrm{mM} \mathrm{Na}$-citrate, $27 \mathrm{mM} \mathrm{Na}$-acetate, $23 \mathrm{mM} \mathrm{Na}$ gluconate, pH 7.0; Fresenius Hemocare, Emmer-Compascuum, the Netherlands) and $30 \%$ plasma, with a sterile connection device (Terumo, Leuven, Belgium). The pooled preparation was centrifuged at $800 \times g$ for 20 minutes to remove RBCs and filtered to remove WBCs. Pooled platelet preparations were stored at $22^{\circ} \mathrm{C}$ in a lateral shaking device. When indicated, apyrase $(0.1 \mathrm{U} / \mathrm{mL})$ was added to the platelet pools containing $A S$, to degrade released $A D P$.

\section{Aggregation}

On day 4 samples were withdrawn from the pooled platelet products, with a sterile connection device and a sample container (Fresenius Hemocare). Platelets stored in full plasma or in 30\% plasma and $70 \%$ AS were counted with a cell counter (AcT-diff, Beckman Coulter) and adjusted with platelet-free autologous plasma or AS, respectively, to a final concentration of $2.5 \times 10^{8}$ platelets $/ \mathrm{mL}$. Autologous platelet-free plasma was obtained by centrifugation of the plasma product twice at $4000 \times g$ for 10 minutes. Where indicated, platelets in AS were centrifuged, washed with Hepes buffer, pH $6.6(136 \mathrm{mM} \mathrm{NaCl}$, 
$2.7 \mathrm{mM} \mathrm{KCl}, 5 \mathrm{mM}$ Hepes, $2 \mathrm{mM} \mathrm{MgCl}$, $10 \mathrm{mM}$ glucose, $0.1 \% \mathrm{BSA}$ ), and finally resuspended in platelet-free autologous plasma to a concentration of $2.5 \times 10^{8}$ platelets $/ \mathrm{mL}$. Platelet-rich plasma (PRP) was incubated with $20 \mu \mathrm{M}$ PPACK (to avoid activation of thrombin substrates, including fibrin formation and platelet activation) before recalcification with $16.7 \mathrm{mM} \mathrm{CaCl}_{2}$. This resulted in a final free $\mathrm{Ca}^{2+}$ concentration of approximately $2.5 \mathrm{mM}$. Platelet aggregations were performed on a dual-channel aggregometer as described (Chronolog, Kordia, Leiden, the Netherlands). ${ }^{184}$ Agonists were added to the platelet suspension after a 5- or 60-minute incubation period at $37^{\circ} \mathrm{C}$, and aggregation was measured as the percentage of change in optical density. The aggregation amplitude (\%) was determined with computer software (Aggrolink software, version 5.1.9, Kordia).

\section{Flow cytometry}

P-selectin was measured as described before with slight changes. ${ }^{100}$ Briefly, PRP $\left(3 \times 10^{8}\right.$ platelets $\left./ \mathrm{mL}\right)$ was diluted and incubated with FITC-labeled MoAb against $P$-selectin (1:50 dilution) for 10 minutes. Samples were fixed with $0.1 \%$ paraformaldehyde and measured in a flow cytometer (Coulter Epics XL, Beckman Coulter). Percentages of platelets positive for P-selectin were determined and compared to a sample of fresh platelets stimulated with TRAP $(15 \mu \mathrm{M}) .{ }^{100} \mathrm{IgG}_{1}$-FITC was used as isotypic control.

\section{Perfusion experiments with reconstituted whole blood}

For perfusion experiments, 9 volumes of blood was freshly drawn into 1 volume of CPD. The blood was centrifuged at $180 \times g$ for 15 minutes, and RBCs were kept aside. To prepare reconstituted whole blood, RBCs were washed as described previously. ${ }^{185}$ In short, 5 volumes of platelets in plasma or AS was mixed with 1 volume of acid citrate dextrose $(80 \mathrm{mM}$ trisodium citrate, $52 \mathrm{mM}$ citric acid, and $180 \mathrm{mM}$ glucose) and centrifuged at $2800 \times \mathrm{g}$ for 2 minutes. The platelet pellet was resuspended in plasma, incubated for 60 minutes at $37^{\circ} \mathrm{C}$, and then mixed with washed RBCs to a final concentration of $2 \times 10^{8}$ platelets $/ \mathrm{mL}$ and a hematocrit level of $37 \pm 2 \%$. The blood was 
perfused for 4 minutes over a collagen-coated coverslip through a parallel-plate flow chamber at a shear rate of $1000 / \mathrm{s}$, as described. ${ }^{88}$ After removal of the non-bound cells by a 5-minute perfusion with Hepes buffer, $\mathrm{pH} 7.5$, highresolution microscopic phase-contrast images were recorded with a digital imaging system (Visitech, Sunderland, UK). Surface area coverage with platelets was analyzed from at least 10 different microscopic fields with computer software (ImagePro, Media Cybernetics, Silver Spring, MD).

This whole-blood perfusion model has been shown to be sensitive for involvement and activation of the platelet-adhesive receptors for collagen and von Willebrand factor, glycoprotein $\mathrm{VI}$, integrins $\alpha_{2} \beta_{1}$ and $\alpha_{\| 1 \mathrm{~b}} \beta_{3}$, and glycoprotein Ib-V-IX. ${ }^{9,88,90}$ Moreover, platelet aggregation achieved is by involvement of the autocrine mediators ADP and thromboxane. ${ }^{186}$

\section{High-performance liquid chromatography analysis}

CPD full plasma or AS samples $(75 \mu \mathrm{L})$, with $30 \%$ plasma, were deproteinated with $20 \% \mathrm{HClO}_{4}(25 \mu \mathrm{L})$ and centrifuged at $20,000 \times \mathrm{g}$ for 10 minutes. Supernatants were neutralized with $400 \mu \mathrm{L}$ of $1 \mathrm{M}$ phosphate buffer and delipidated by extraction with $500 \mu \mathrm{L}$ of $50 \% n$-heptane. Resulting samples were used for high-performance liquid chromatography (HPLC) detection of ADP, basically as described. ${ }^{163}$ Aliquots of $20 \mu \mathrm{L}$ were injected into a Supelcosil LC18 column $(7.5 \mathrm{~cm} \times 4 \mathrm{~mm}, 3 \mu \mathrm{m}$; Sigma). ADP was separated from other nucleotides with gradient elution of Buffer A with methanol (Solvent B) at a flow rate of $1 \mathrm{~mL}$ per minute: first methanol was maintained at $0.5 \%$ for 9 minutes and then it was increased to $20 \%$ for 1 minute, followed by a plateau level of $20 \%$ methanol for the next 4 minutes. Methanol was then decreased to $0.5 \%$ for 1 minute and kept at this level during a post-run period of 4 minutes. Buffer $A$ consisted of $6 \mathrm{mM}$ tetrabutylammonium phosphate in $0.1 \mathrm{M}$ phosphate buffer ( $\mathrm{pH}$ 6.0). ADP was monitored at $260 \mathrm{~nm}$ and quantified by integration of peak absorbance areas, employing a standard curve constructed with ADP at 10 to $200 \mu \mathrm{M}$ concentrations. ADP eluted within a 14-minute run. 


\section{Statistical analysis}

When appropriate, a non-parametric test for two dependent (Wilcoxon test) or independent variables (Mann-Whitney $U$ test) was applied. Differences were considered significant at a $p$-value of less than 0.05. Data are expressed as mean \pm SEM.

\section{Results}

\section{Involvement of ADP in agonist-induced platelet aggregation}

Platelet aggregation in fresh PRP was assessed by activation with collagen $(5 \mu \mathrm{g} / \mathrm{mL})$, TRAP $(5 \mu \mathrm{M})$, arachidonic acid $(0.5 \mathrm{mg} / \mathrm{mL})$, thromboxane $A_{2}$ analog $\mathrm{U} 46619(70 \mu \mathrm{M})$, or ADP $(10 \mu \mathrm{M})$. To establish the contribution of autocrine ADP in the aggregation, the platelets were activated by these agonists in the absence or presence of ADP degrading apyrase $(1 \mathrm{U} / \mathrm{mL})$. As depicted in figure 1 , this amount of apyrase was sufficient to completely antagonize the aggregation induced by $10 \mu \mathrm{M}$ ADP. Notably, we observed a significant decrease in aggregation responses with all agonists in the presence of apyrase. This confirms the notion that platelet aggregation induced by many platelet agonists is dependent on secondary activation due to autocrine released ADP. ${ }^{152}$ Typically, the collagen-induced signaling pathway was most sensitive to ADP under these conditions, in agreement with published findings; ${ }^{187-189}$ maximal aggregation evoked by collagen was reduced from $85 \pm 4$ to $22 \pm 9 \%$ in the presence of apyrase. We note that in the presence of apyrase, aggregation triggered by the various agonists can be fully restored when the stable analog 2MeSADP was added to the reactions (data not shown).

\section{P-selectin expression of platelets stored in different media}

Surface exposure of P-selectin (CD62) is considered to be a temporary marker of platelet secretion and, thus, platelet activation. To determine the state of platelet activation after 1, 4, and 7 days of storage in autologous plasma, PAS-II, or Composol, we examined this variable. In agreement with earlier findings, ${ }^{98,129,190-192}$ we found on day 1 a significant increase in P-selectin expression for platelets that 


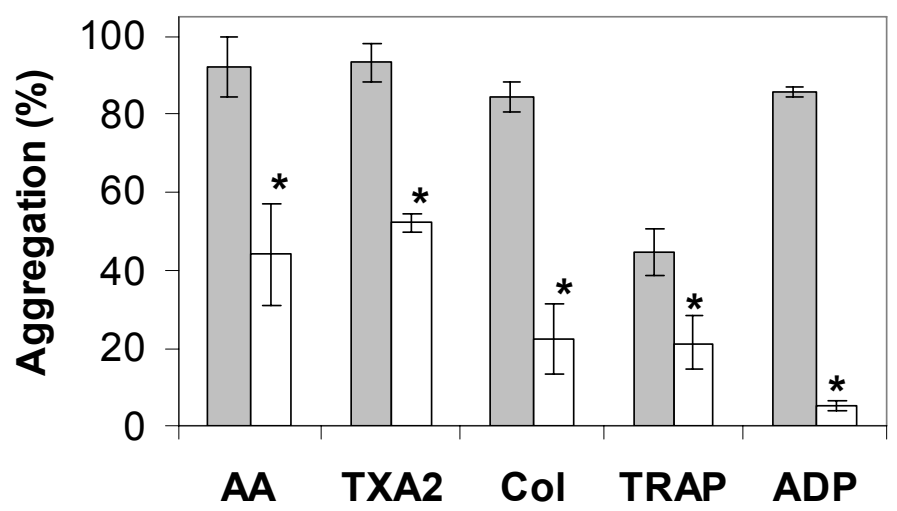

Figure 1. Contribution of granular ADP to platelet aggregation induced by different agonists. Maximal platelet aggregation in fresh citrated PRP $\left(2.5 \times 10^{8} / \mathrm{mL}\right)$ in the absence $(\boldsymbol{m})$ and presence (口) of $1 \mathrm{U} / \mathrm{mL}$ apyrase. Aggregation was triggered after recalcification in the presence of PPACK $(20 \mu \mathrm{M})$ by the agonists arachidonic acid, $0.5 \mathrm{mg} / \mathrm{mL}(\mathrm{AA})$.

were stored in autologous plasma $(16.1 \pm 1.1 \% ; n=14)$, PAS-II $(33.3 \pm 1.3 \%$; $\mathrm{n}=14)$, or Composol $(19.8 \pm 1.7 \%, \mathrm{n}=14)$ in comparison to fresh platelets (7.4 $\pm 0.3 \% ; n=6 ; p<0.001$ ), indicating appreciable release of granule contents in all three storage solutions (table 1). Typically, P-selectin expression was also significantly increased for platelets stored in PAS-II compared to platelets in autologous plasma after a 7-day storage period. This was not the case for platelets stored in Composol. These results thus point to considerable granule secretion, especially during storage in PAS-II medium, that is, without $\mathrm{Mg}^{2+}$ en $\mathrm{K}^{+}$ions.

Table 1. Percentage of P-selectin-positive platelets in plasma, PAS-II, or Composol during storage*

\begin{tabular}{clll} 
Storage time & Plasma & PAS-II & Composol \\
\hline Fresh & $7.4 \pm 0.3(6)$ & & \\
Day 1 & $16.1 \pm 1.1(14)$ & $33.3 \pm 1.3(14) \ddagger$ & $19.8 \pm 1.7(14)$ \\
Day 4 & $17.2 \pm 1.2(14)$ & $37.4 \pm 1.1(14) \ddagger$ & $19.4 \pm 0.9(14)$ \\
Day 7 & $22.1 \pm 0.9(14) \dagger$ & $41.5 \pm 1.0(14) \dagger \ddagger$ & $22.7 \pm 1.0(14)$ \\
\hline
\end{tabular}

*Values are expressed as means \pm SEM (number)

† Day 1 vs day $7, p<0.001$

$\ddagger$ Plasma vs PAS-II, $p<0.001$ 


\section{Effect of storage medium on ADP-induced platelet aggregation}

Aggregation of platelets in plasma, PAS-II, and Composol after 3 or 4 days of storage was induced by addition of a submaximal dose of ADP $(10 \mu \mathrm{M})$. Figure $2 \mathrm{~A}$ shows that, after a 5 -minute incubation period at $37^{\circ} \mathrm{C}, \mathrm{ADP}$-induced aggregation was almost absent for platelets in PAS-II or Composol, whereas platelets in plasma showed a high aggregation response. After 60 minutes of incubation at $37^{\circ} \mathrm{C}$, the response to ADP slightly increased for platelets suspended in PAS-II or Composol, but these were still significantly lower than those of platelets stored in plasma (figure 2B). This points to a loss of ADP responsiveness by storage in AS, possibly due to the absence of permissive factors in plasma.

To investigate whether a decreased ADP receptor function could be recovered, we resuspended platelets that had been stored for 4 days in PAS-II

A

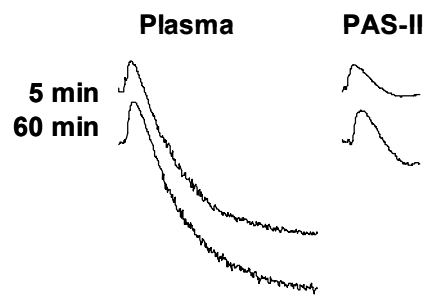

\section{PAS-II(p)}

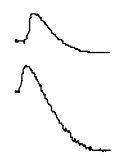

Composol Composol(p)
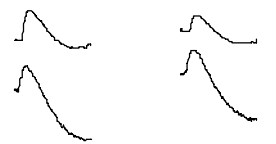

$20 \%$

B

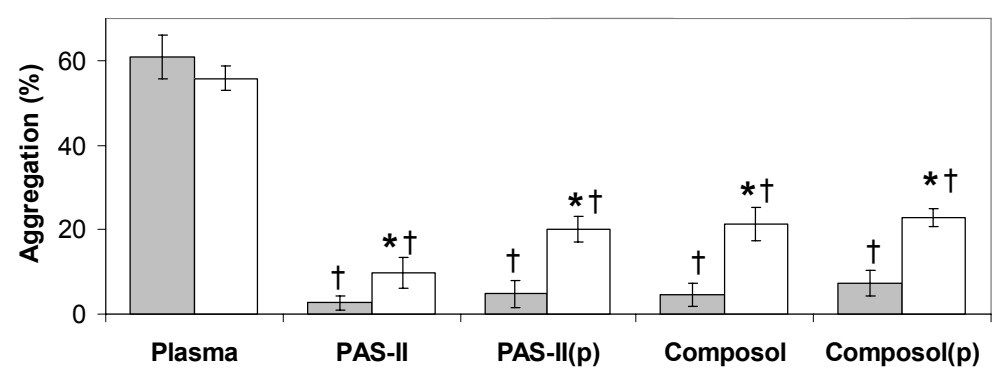

Figure 2. ADP-induced aggregation of platelets stored in plasma, PAS-II, and Composol. Platelets were stored for 4 days under blood bank conditions. Platelets in plasma, PAS-II, or Composol or platelets that were sedimented from ASs and resuspended in plasma ( $p$ ) were incubated at $37^{\circ} \mathrm{C}$ for 5 (-) or 60 (口) minutes, as indicated. Subsequently, PRP or AS was recalcified in the presence of PPACK $(20 \mu \mathrm{M})$ and aggregation was induced with $10 \mu \mathrm{M}$ ADP. Representative traces are depicted in $A$ and maximal aggregation (mean \pm SEM) of seven independent experiments is shown in $B$. ${ }^{*} p<0.05$, compared to 5 minutes; $\dagger p<0.05$, compared to plasma. 
or Composol again in full plasma. Control experiments indicated that processing of reactive platelets (sedimentation and re-uptake in plasma) did not affect their responsiveness (data not shown). The fact that re-uptake in plasma of platelets that had been stored in PAS-II or Composol did not affect ADP-induced aggregation after a 5 -minute incubation at $37^{\circ} \mathrm{C}$ indicates that differences found in this assay between platelets stored in plasma and AS were not caused by dissimilar amounts of plasma in the assay, as has previously been suggested for other platelet function assays. ${ }^{193}$

Re-uptake in plasma of platelets that had previously been stored in PAS-II or Composol resulted in a slight, but significant, recovery of ADP-induced platelet aggregation, following a 60 -minute incubation at $37^{\circ} \mathrm{C}$. On average, the aggregation reaction remained lower than that of platelets that were stored in full plasma (figures $2 \mathrm{~A}$ and $2 \mathrm{~B}$ ).

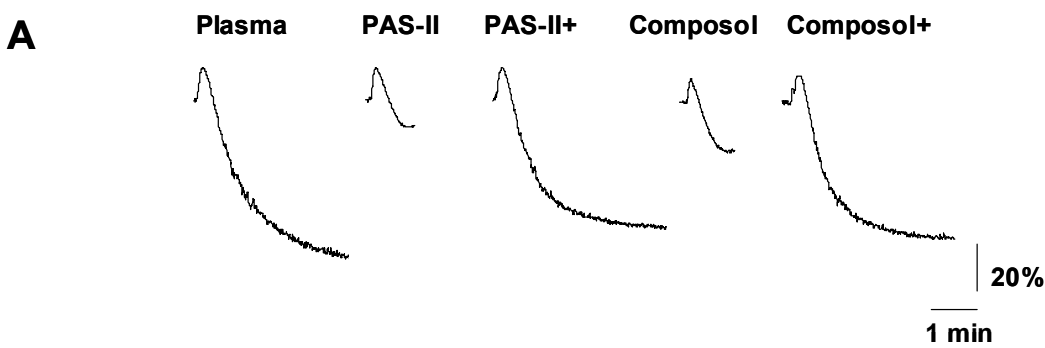

B

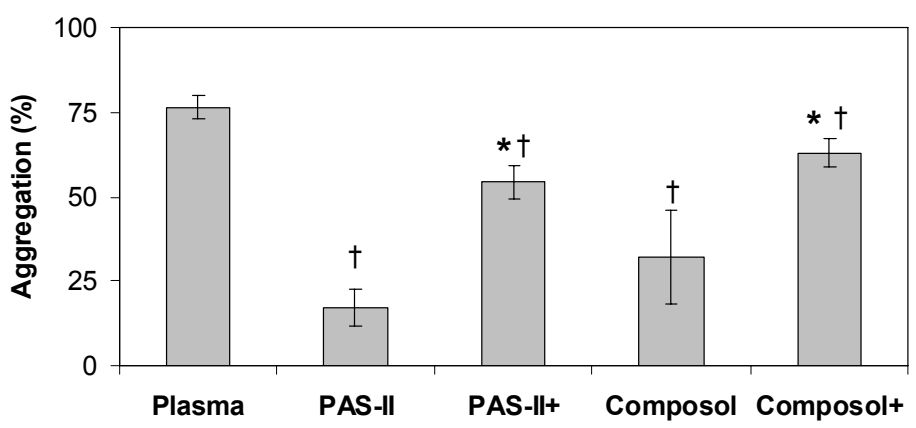

Figure 3. Addition of apyrase to platelets in PAS-II or Composol preserves ADP-induced aggregation. Platelets were stored in plasma, PAS-II, or Composol in the presence or absence of $0.1 \mathrm{U} / \mathrm{mL}$ apyrase for 4 days under blood bank conditions. Platelets were incubated at $37^{\circ} \mathrm{C}$ for 5 minutes, after which PRP or AS was recalcified in the presence of PPACK $(20 \mu \mathrm{M})$. Aggregation was induced with $5 \mu \mathrm{M} 2 \mathrm{MeSADP}$, a stable ADP analog, to prevent degradation by apyrase. Representative traces are depicted in $A$, and maximal aggregation (mean \pm SEM) of seven independent experiments is shown in B. +Storage in the presence of apyrase. ${ }^{*} p<0.05$, compared to absence of apyrase; $\uparrow p<0.05$, compared to plasma. 
To establish whether the preserving effect of storage in plasma was related to ADP degrading activity, we added the ADPase apyrase $(0.1 \mathrm{U} / \mathrm{mL})$ to platelets that were resuspended in the presence of $70 \%$ PAS-II or Composol solution directly after their preparation. After a 4-day storage period, we stimulated these platelets with the non-degradable ADP analog 2MeSADP, which avoids breakdown of the agonist by apyrase. Figure 3 shows that the ADP-induced platelet aggregation was increased by addition of apyrase. To further support the notion that ADP can be degraded in plasma, we measured the conversion of ADP into AMP by HPLC analysis in undiluted plasma and in plasma containing $70 \%$ PAS-II or Composol. At $37^{\circ} \mathrm{C}$, added ADP (1 mM) was degraded at approximately 2.6 times higher rate in plasma with respect to synthetic media (data not shown).

A

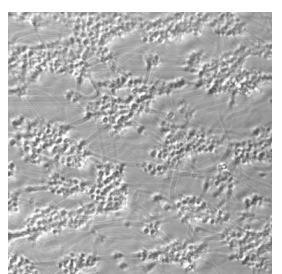

Plasma

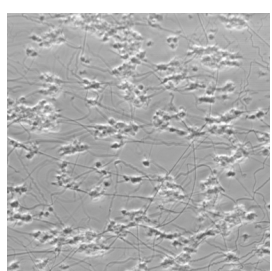

PAS-II

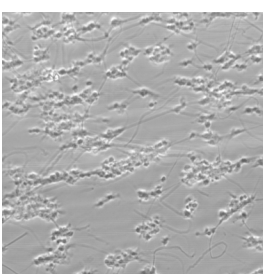

Composol

B

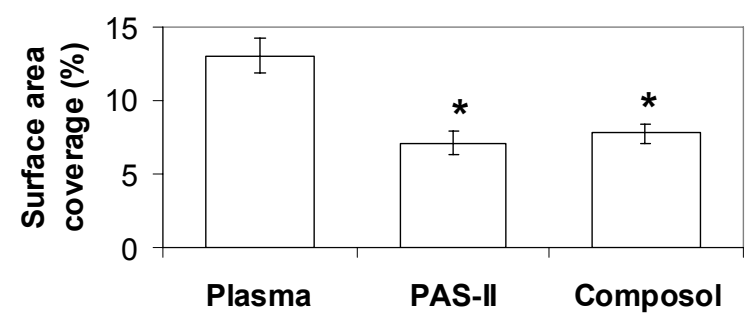

Figure 4. Shear-induced adhesion of platelets stored in plasma, PAS-II, or Composol to a collagen surface. Platelets stored for 4 days in plasma, PAS-II, or Composol were reconstituted with RBCs and plasma, as described under Materials and methods. After a 60-minute incubation period at $37^{\circ} \mathrm{C}$, the reconstituted blood was perfused over collagen at a shear rate of $1000 / \mathrm{s}$ for 4 minutes. Shown are representative images at the collagen surfaces after perfusion (A). Platelet surface coverage was calculated from at least 10 different microscopic fields. Bars represent mean \pm SEM of at least four independent experiments (B). ${ }^{*} p<0.05$, compared to condition with plasma. 


\section{Adhesion of stored platelets to collagen under flow conditions}

The findings thus far indicate that ADP responsiveness of platelets stored in AS such as PAS-II and Composol deteriorates. We determined the ability of these platelets to participate in thrombus formation on a collagen surface at arterial blood flow conditions (shear rate, 1000/s). Thrombus formation in this ex vivo flow model is known to rely on autocrine ADP release. ${ }^{88,194}$ In an attempt to mimic transfusion conditions, platelets stored for 4 days in plasma, PAS-II, or Composol were resuspended in full plasma, incubated for 60 minutes at $37^{\circ} \mathrm{C}$, and then reconstituted with RBCs before start of perfusion. Figure 4A depicts the differences in thrombi formation after perfusion of this reconstituted blood. Thrombus formation was reduced for platelets that were stored in PAS-II or Composol with respect to platelets stored in full plasma. Quantitative analysis indicated that the percentage of surface coverage was significantly lower for platelets that were stored in PAS-II or Composol (figure 4B). These observations suggest that the hemostatic activity of platelets stored in PAS-II or Composol is reduced and that this loss cannot be regained by the 60 -minute treatment in plasma at $37^{\circ} \mathrm{C}$.

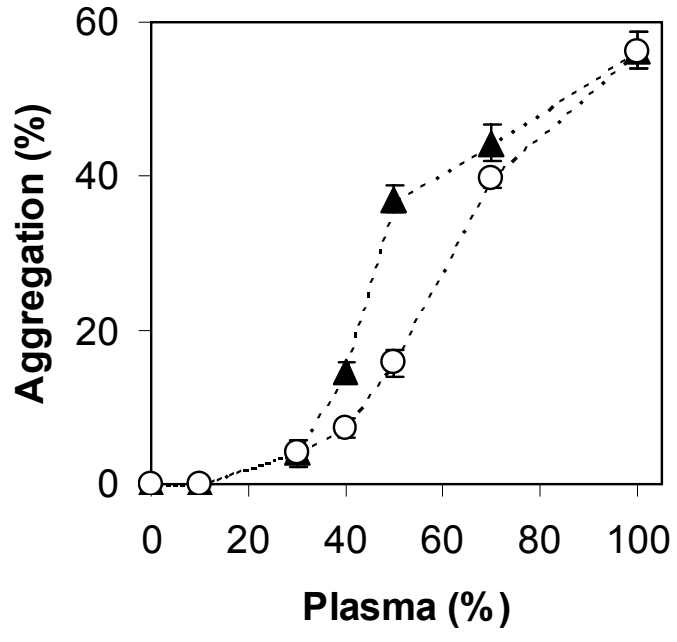

Figure 5. Effect of plasma concentration during storage on ADP-induced platelet aggregation. Platelets were stored in PAS-II (०) or Composol $(\boldsymbol{\Delta})$ with varying amounts of plasma $(0 \%$ $100 \%$ ). After a 3- to 4-day storage period in these solutions, the platelets were incubated at $37^{\circ} \mathrm{C}$ for 5 minutes and subsequently recalcified in the presence of PPACK $(20 \mu \mathrm{M})$. Aggregation was then induced with $10 \mu \mathrm{M}$ ADP. Shown are means \pm SEM of three independent experiments. 


\section{Storage of platelets in different plasma-to-AS ratios and effects on ADP- induced platelet aggregation}

Finally, we determined which amount of AS affected the platelet responses to ADP. Therefore, platelets were suspended in plasma containing various fractions of PAS-II or Composol and stored for 4 days under blood bank conditions. Subsequently, we measured aggregation with $10 \mu \mathrm{M}$ ADP. We noted a gradual increase in aggregation response along with the increase in plasma content, with (figure 5) maximal ADP-induced responses obtained at $100 \%$ plasma. Typically, the aggregability was maintained at a level greater than approximately $65 \%$ after storage in $50 \%$ Composol or $30 \%$ PAS-II. Apparently, platelets stored in PAS-II need higher amounts of plasma for conservation of their ADP response than platelets in Composol.

\section{Discussion}

Although a number of studies has assessed the in vitro quality of platelet concentrates stored in synthetic media by metabolic tests, ${ }^{173-175}$ very little is known about the activation function of platelets stored in such media and their clinical functionality. In this study, we determined in vitro functionality, and in particular ADP responsiveness, of platelets stored at reduced plasma content $(30 \%)$ in two different synthetic media, namely, PAS-II and Composol. As confirmed in this article, platelet activation by various physiologic agonists depends on autocrine effects induced by ADP released from the dense granules. ${ }^{151,152}$ This underscores the importance of autocrine ADP-mediated signaling in the hemostatic function of platelets. We found that platelets stored for 3 to 4 days in either AS with reduced plasma content (30\%) lost their ability to aggregate in response to ADP. This is likely due to rapid desensitization of ADP receptors after release of granular ADP during storage. ${ }^{115,195,196}$ In agreement with this, we found that platelets stored in PAS-II or Composol show reduced collagen-induced thrombus formation under arterial flow conditions, which process highly relies on autocrine ADP secretion and ADP receptor activation. $^{37,194}$ 
The expression of P-selectin on the surface of platelets is a good indicator for degranulation. ${ }^{197}$ Circulating degranulated platelets can lose P-selectin, but continue to circulate and function, implying that $P$-selectin is not associated with survival after transfusion. ${ }^{198}$ We confirm the results of others ${ }^{98,129,190-192}$ that already after 1 day of storage platelets show a significant exposure of $P$ selectin, regardless of whether they are stored in plasma, PAS-II, or Composol, pointing to perhaps continuous granule release during preparation and storage. We also find, similarly to what has been published by others, that P-selectin expression of platelets in PAS-II is doubled with respect to storage in autologous plasma. ${ }^{180,199,200}$ We furthermore found that exposure of P-selectin was limited upon storage in the presence of Composol, containing millimolar concentrations of $\mathrm{Mg}^{2+}$ and $\mathrm{K}^{+}$ions, suggesting that these cations are required to suppress spontaneous granule secretion. Reduced secretion with $\mathrm{Mg}^{2+}$ and $\mathrm{K}^{+}$ions has also been described by others. ${ }^{176,177,181}$

The finding that platelets stored in Composol become more refractory for ADP stimulation than platelets stored in plasma seemingly contradicts with the P-selectin data: the latter suggest that release of granular ADP is similar for platelets in Composol and plasma. This apparent discrepancy, however, is explained by evidence that stored plasma contains high ecto-nucleotidase (ADPase) activity, which degrades autocrine-released ADP and therefore antagonizes desensitization of the platelet ADP response. ${ }^{116}$ The presence of ADPase activity in stored plasma is confirmed in this study by HPLC analysis. We also demonstrated that addition of the ADPase apyrase to platelets in PASII or Composol preserves ADP responses.

Although the P-selectin data indicate that granule secretion is higher during storage of platelets in PAS-II than in Composol, we find that ADP responses become severely diminished in both media. This implies that the amount of ADP that is released from platelets stored in Composol with $30 \%$ plasma content is already sufficient for desensitization of ADP responses. We showed that the presence of $30 \%$ plasma is too low to preserve platelet ADP responses, and $100 \%$ plasma is necessary to fully preserve ADP responsiveness. 
Various studies have suggested that a decreased activation tendency of platelets during storage can improve in the circulation after transfusion, ${ }^{96,131,196,201,202}$ pointing to partial reversibility of storage-induced lesions. ${ }^{201,202}$ Unfortunately, no golden standard is yet available to predict and evaluate platelet function in vivo and the success of a platelet transfusion is currently only based on platelet number rather than platelet function. We determined whether the reduced ADP responsiveness of platelets stored in AS is potentially reversible after resuspension in plasma, for example, upon transfusion. This appeared to be the case, because resuspension of desensitized platelets in full plasma and incubation at $37^{\circ} \mathrm{C}$ resulted in significantly improved ADP function. After storage in the AS, however, the ADP responses only partly $(30 \%)$ recovered, implying that a substantial part of the refractoriness is irreversible. The decreased reactivity of platelets stored in synthetic media is further confirmed in a perfusion model, where stored platelets were reconstituted to whole blood and after a 60 -minute incubation at $37^{\circ} \mathrm{C}$ were perfused over collagen surfaces. Thrombus formation under these conditions is highly dependent on ADP release and ADP receptor signaling. ${ }^{88,194}$ We observed that platelet thrombus formation was severely reduced for platelets that were stored in PAS-II and Composol, which is in complete agreement with the evidence for ADP receptor dysfunction.

In summary we conclude that in ASs with low plasma content (30\%), platelet ADP responses are severely diminished and only partly recover in the presence of full plasma or blood. Storage at higher plasma content leads to a better preservation of ADP responsiveness. Likely because of increased secretion in the presence of PAS-II, more plasma is needed to preserve ADP receptor function than in the presence of Composol.

Acknowledgment: We acknowledge G. Hageman, PhD, for her help with HPLC analysis. This study was financially supported by the Sanquin Blood Bank South East Region. 


\title{
Chapter 5
}

\author{
Shedding of \\ procoagulant microparticles from \\ unstimulated platelets by \\ integrin-mediated destabilization of \\ actin cytoskeleton
}

\author{
Sandra Cauwenberghs, Marion A.H. Feijge, \\ Alan G.S. Harper, Stewart O. Sage, Joyce Curvers and \\ Johan W.M. Heemskerk \\ FEBS Letters \\ 2006; 580:5313-20
}

Reprinted with permission 


\section{Abstract}

Platelet activation by potent, $\mathrm{Ca}^{2+}$-mobilizing agonists results in shedding of microparticles that are active in coagulation. Here we show that platelets under storage produce procoagulant microparticles in the absence of agonist. Microparticle formation by resting platelets results from allb $\beta 3$ signaling to destabilisation of the actin cytoskeleton in the absence of calpain activation. Integrin-mediated spreading of platelets over fibrinogen similarly results in microparticle formation. After transfusion of stored platelet preparations to thrombocytopenic patients, the microparticles contribute to coagulant activity in vivo. 


\section{Introduction}

Platelets contribute to hemostasis and thrombosis by assembling into aggregates and by stimulating blood coagulation. It is generally assumed that, to become procoagulant, platelets need to be stimulated with strong agonists, e.g. $\mathrm{Ca}^{2+}$ ionophore or the combination of collagen and thrombin. ${ }^{51,203}$ In a $\mathrm{Ca}^{2+}$ dependent response similar but non-identical to apoptosis, these agents cause surface exposure of negatively charged phospholipids, such as phosphatidylserine (PS). This process enhances the assembly of coagulation factor complexes, and leads to the formation of factor Xa and thrombin and, hence, to coagulation.

For over twenty years, it is known that the same strong agonists also stimulate platelets to shed microparticles (MP), via $\mathrm{Ca}^{2+}$ influx and activation of the $\mathrm{Ca}^{2+}$-dependent protease calpain, which structures also express procoagulant PS at their outer surface. ${ }^{47,204-206}$ This shedding of MP, also described for other cells of the cardiovascular system, is likely to be of physiological relevance, as elevated MP levels in plasma associate with cardiovascular disease and thrombotic complications. ${ }^{207,208}$

The integrin allbß3 (>80,000 copies per platelet) plays a well established role in platelet aggregate and plug formation. It signals in both directions over the plasma membrane. Following platelet stimulation, signaling from the inside results in an active integrin conformation, which is required for binding of fibrinogen and von Willebrand factor. In addition, once activated, the integrin transmits still poorly understood outside-in signals in platelets, which include Src kinase and phosphoinositide 3-kinase activity, and actin cytoskeleton modification via cofilin and filamin. ${ }^{38,45,209,210}$ Integrin outside-in signaling is known to mediate actin rearrangements that, for instance, cause platelets to spread over an adhesive surface and to retract, when encapsulated within a clot. $^{211}$

Preparations of platelets that are stored under blood bank conditions and used for transfusion purposes, appear to be enriched in MP with high coagulant activity. ${ }^{100}$ How such MP are formed by stored platelets has not been investigated. In the present study, we show that resting platelets 
'spontaneously' shed procoagulant MP via a mechanism that involves integrin allbß3 signaling towards cytoskeleton remodeling, in the apparent absence of integrin activation and platelet aggregation. This pathway differs from that of agonist-induced MP formation in the way that it does not require $\mathrm{Ca}^{2+}$ elevation and calpain activity. The produced MP appear to be active in coagulation, when transfused together with stored platelets into thrombocytopenic patients.

\section{Materials and Methods}

\section{Materials}

Abciximab (reopro) was from Centocor (Leiden, NL), tirofiban (aggrastat) from Merck (Haarlem, NL), eptifibatide (integrilin) and lotrafiban from GlaxoSmithKline (Zeist, NL), (labeled) annexin A5 from Nexins Research (Hoeven, NL) and iloprost from Schering (Berlin, G). Fluorescein isothiocyanate (FITC)-labeled anti-Pselectin mAb was from Immunotech (Marseille, F), while FITC (PE)-anti-CD61 (anti-B3) $\mathrm{mAb}$ and isotype-matched IgG1 were from BD Biosciences (San Jose, CA). Fura-2/AM, DM-BAPTA1 AM and Alexa Fluor phalloidin were from Molecular Probes (Leiden, NL), jasplakinolide was from VWR (Amsterdam, NL). PP1-3 and calpeptin were from Calbiochem (San Diego, CA), cytochalasin $D$ and latrunculin B from Sigma (St. Louis, MO). The fluorogenic calpain substrate, tBoc-Leu-Met chloroamino methylcoumarin (tBoc-LM-CMC), was from Invitrogen (Paisley, UK). Sources of other reagents are described elsewhere. ${ }^{116}$

\section{Blood collection and platelet preparation}

Blood was drawn after full consent by venapuncture from healthy volunteers, free of medication. ${ }^{158}$ Blood was also obtained from a thrombocytopenic patient with myeloproliferative disorder both before and shortly after platelet transfusion. ${ }^{212}$ Experiments were approved by the local Medical Ethics Committee. Platelet-rich plasma (PRP) was collected by centrifuging blood at $240 \mathrm{~g}$ for $15 \mathrm{~min}$; leukocyte count was $<1 \times 10^{3} / \mathrm{mL}$. Washed platelets were prepared from PRP ${ }^{158}$, and resuspended at $4 \times 10^{8}$ platelets $/ \mathrm{mL}$ in Hepes buffer $\mathrm{pH} 7.45$ (136 mM NaCl, $10 \mathrm{mM}$ Hepes, $2.7 \mathrm{mM} \mathrm{KCl}, 2 \mathrm{mM} \mathrm{MgCl}$, $0.1 \%$ bovine serum albumin and $0.1 \%$ glucose). 


\section{Platelet incubation and aggregation}

PRP or washed platelets, normalized at $4 \times 10^{8}$ platelets $/ \mathrm{mL}$, was slowly rotated $(10 \mathrm{rpm})$ at room temperature in closed, air-transparent tubes for up to $24 \mathrm{~h}$. Inhibiting or activating agents were added, as described. At indicated times, MP-containing platelet-free plasma (PFP) was obtained by centrifuging $0.5 \mathrm{~mL}$ samples of the PRP at $20,000 \mathrm{~g}$ for $10 \mathrm{~min}$. Platelet count in the PFP was below detection $\left(<1 \times 10^{5} / \mathrm{mL}\right)$, while loss of MP was about $20 \%$.

Aggregation of platelets in PRP was determined under stirring at $37^{\circ} \mathrm{C}$ by conventional aggregometry. ${ }^{213}$ Citrate anti-coagulated PRP was recalcified with 16.6 $\mathrm{mM} \mathrm{CaCl}_{2}$ in the presence of Phe-Pro-Arg chloromethyl ketone (PPACK, $10 \mu \mathrm{M})$, to achieve $\mathrm{mM}$ concentrations of free $\mathrm{Ca}^{2+}$ and $\mathrm{Mg}^{2+}$ without coagulation. ${ }^{116}$

\section{Flow cytometry}

Platelet activation was measured by flow cytometry, using FITC-labeled mAbs against P-selectin or activated allbß3 (PAC1). ${ }^{100}$ FITC-annexin A5 was used to detect exposed PS. Platelet-derived MP in plasma were measured after dilution with anti-coagulated, filtered Hepes buffer $\mathrm{pH}$ 7.45. After 15 min labeling with PE-anti- $\beta 3 \mathrm{mAb}$, the MP were detected as fluorescent events with low forward and side scatter positively staining for $\alpha \mathrm{llb} \beta 3$. Fluorescent beads were used for calibration.

\section{Measurement of thrombin generation in plasma}

Platelet-dependent thrombin generation was measured in PRP. ${ }^{102}$ Assay conditions were $1 \times 10^{8}$ platelets $/ \mathrm{mL}, 67 \%$ citrate plasma and $33 \%$ buffer $\mathrm{A}$ (140 $\mathrm{mM} \mathrm{NaCl}, 20 \mathrm{mM}$ Hepes, $0.5 \%$ bovine serum albumin, $\mathrm{pH}$ 7.45). Reactions were started by addition of $1 \mathrm{pM}$ (f.c.) tissue factor, $16.6 \mathrm{mM} \mathrm{CaCl}_{2}$ and $415 \mu \mathrm{M}$ fluorescent substrate Z-Gly-Gly-Arg aminomethyl coumarin. Similar reaction conditions were used to measure MP-dependent thrombin generation, but now using centrifuged (MP-containing) PFP. Assays were performed in triplicate at $37^{\circ} \mathrm{C}$ using a Fluoroskan Ascent well plate reader. ${ }^{102}$ Controls were always run to exclude interference of (ant)agonists with the coagulation process per se. 


\section{Real-time measurement of calpain activity}

$\mathrm{PRP}$, incubated for up to $24 \mathrm{~h}$ at room temperature, was used to prepare washed platelets in the presence of apyrase. ${ }^{116}$ The washed cells were treated with vehicle of calpeptin $(140 \mu \mathrm{M})$, and loaded with the fluorogenic calpain substrate, $t$ Boc-LM-CMC, for $30 \mathrm{~min}$ at $37^{\circ} \mathrm{C}$, as described. ${ }^{214}$ During the measurements, EGTA (1 $\mathrm{mM})$ was added to prevent possible extracellular calpain activity. Intracellular calpain activity was measured from fluorescence increases due to substrate cleavage by stopped-flow fluorometry (agonists present as shown). ${ }^{214}$ Fluorescence increase in time was corrected for probe autofluorescence at baseline; averaged traces were produced from 3 repeated scans.

\section{Microscopy}

Phase-contrast and (confocal) fluorescence microscopy were performed as described. $^{88}$

\section{Statistical analysis}

Significance of differences was determined with the non-parametric MannWhitney $U$ test.

\section{Results}

\section{Platelets shed procoagulant microparticles at minimal activation}

Triggering of freshly isolated PRP with tissue factor gives a delayed generation of thrombin. ${ }^{5,102}$ Experiments indicated that, when platelets in plasma were incubated under slow rotation at room temperature for up to $24 \mathrm{~h}$ (conditions resembling those of platelet storage), the rate and extent of tissue factor-triggered thrombin generation greatly increased with the time of incubation (figure $1 \mathrm{~A}$ ). To determine the contribution of MP in the increased coagulant activity, incubated PRP samples were centrifuged at a high speed removing all platelets. The supernatant platelet-free plasma (PFP) was also increasingly active in thrombin formation (maximal levels 25-250 nM), after triggering with tissue factor (figure $1 \mathrm{~A}$ ). Control experiments showed that 10 
$\mu \mathrm{g} / \mathrm{mL}$ of PS-blocking annexin A5 completely inhibited thrombin generation, in both the incubated PRP and the centrifuged PFP samples (figure 1A). This indicated that all thrombin generation in plasma relied on the presence of procoagulant PS.

Flow cytometry was used to confirm the formation of MP in the rotated PRP. The MP were identified as small-size scatter events, staining positive for integrin $\beta 3$. Double-staining indicated that most of the $\beta 3$-positive events also stained with fluorescent-labeled annexin A5, pointing to exposed PS (figure 1B).

Prolonged, 6-h incubation of PRP resulted in a progressive accumulation of the MP, although their level remained lower than that of platelets stimulated with strong agonists (figure $1 \mathrm{C}$, left). Interestingly, MP formation was also detected in suspensions of washed platelets that were long incubated in Hepes buffer under rotation. Thrombin generation measurements showed a high correlation between the MP levels, detected by flow cytometry, and the coagulant activity of the centrifuged PFP samples (figure 1C, right). As required, ultraspeed centrifugation of the PFP, to pellet all MP, resulted in completely abolished thrombin generation. Together, these data indicate that prolonged incubation of platelets in either plasma or buffer leads to shedding of MP with high thrombogenic activity.

To investigate how MP shedding influenced the function of platelets, we performed flow cytometric measurements of platelet activation markers. After 6$\mathrm{h}$ incubation of PRP, only 5-8\% of the platelets expressed P-selectin (a marker of exocytosis), activated allbB3 integrin (detected with FITC-PAC1 mAb), or PS (figure $2 \mathrm{~A}$ ). This sharply contrasted the $\sim 100 \%$ of platelets expressing these activation markers after stimulation with the strong agonists convulxin/ADP or ionomycin. Furthermore, the incubated PRP still had high aggregation responses with lower doses of ADP or collagen (figure $2 \mathrm{~B}$ ). It thus appears that the shedding of procoagulant MP is accompanied by minimal platelet activation, and is of little influence on agonist-induced platelet aggregation. 
A
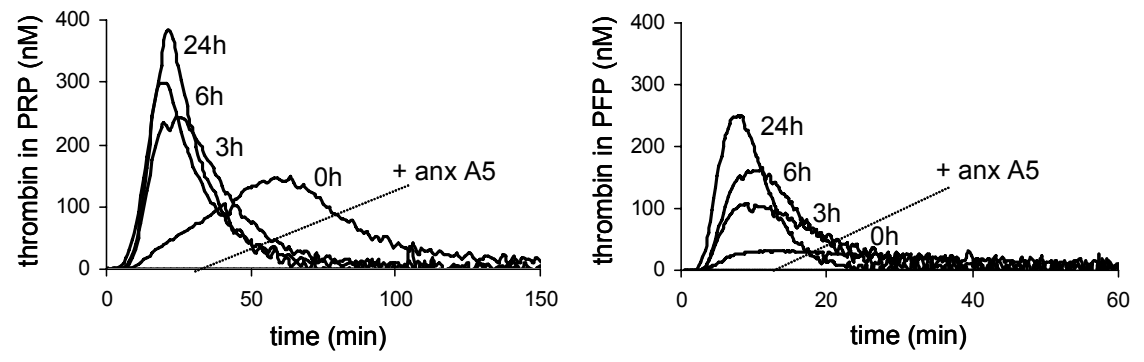

B
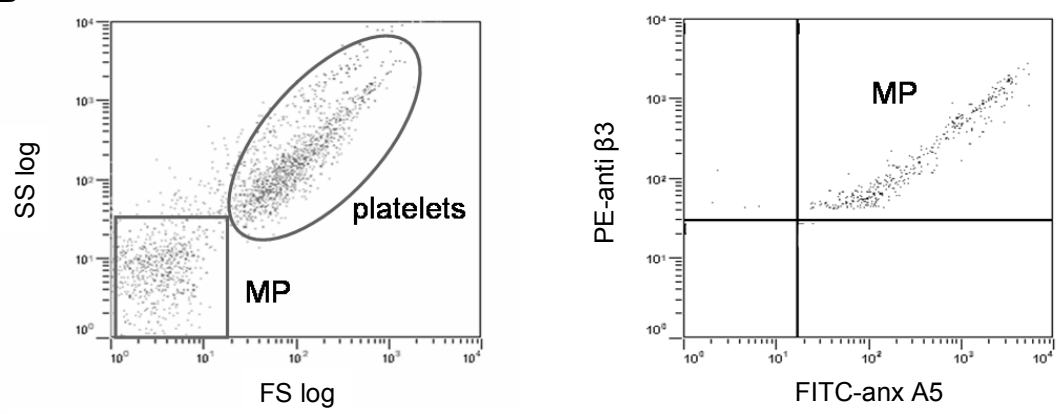

C
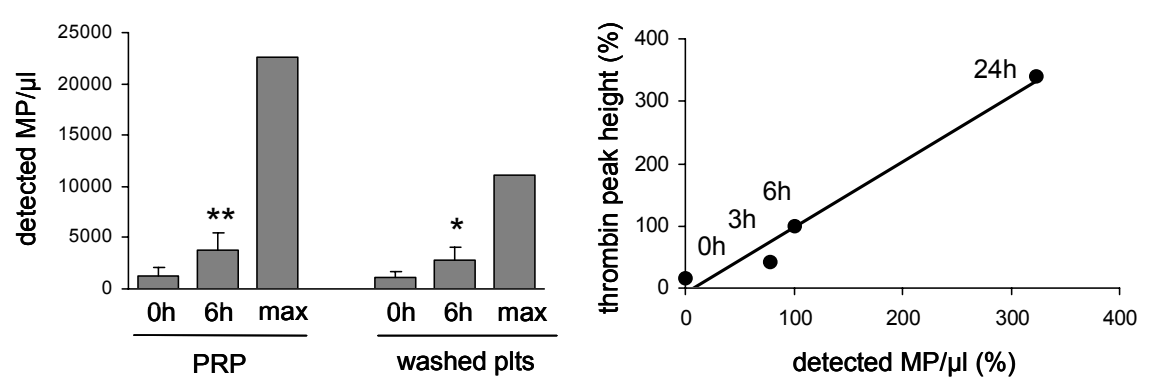

Figure 1. Progressive shedding of procoagulant MP during platelet storage. (A) Left: Tissue factor-induced thrombin generation of PRP that was incubated at room temperature under rotation for 0-24 h. Right: Tissue factor-induced thrombin generation of PFP prepared by centrifuging of the rotated PRP. Annexin A5 $(10 \mu \mathrm{g} / \mathrm{mL})$ was added at $5 \mathrm{~min}$ before tissue factor, where indicated $(6 \mathrm{~h}$ rotation, curves co-incide with $x$-axis). (B) Flow cytometric detection of MP in 6-h rotated PRP. Colabeling with FITC-annexin A5 (FL1) and PE-labeled anti-B3 mAb (FL2). (C) Left: Flow cytometric measurement of MP in PRP and in washed platelet suspension, incubated for 0-6 h. Maximal (max) amounts of MP were formed by stimulation with $50 \mathrm{ng} / \mathrm{mL}$ convulxin and $20 \mu \mathrm{M}$ ADP (PRP), or with $10 \mu \mathrm{M}$ ionomycin (washed platelets). Right: Correlation between MP detected by flow cytometry and MP-derived thrombin generation in plasma $\left(R^{2}=0.97, P=0.013\right)$. Data are means \pm S.E.M. $(n=3-5)$, ${ }^{*} p<0.1$ and ${ }^{* *} p<0.05$, compared to $0 \mathrm{~h}$. 


\section{'Spontaneous' MP formation is mostly independent of calpain but is controlled by allb $\beta 3$}

Early reports indicate that strong platelet agonists activate the $\mathrm{Ca}^{2+}$-dependent protease, calpain, which then plays a regulatory role in MP formation. ${ }^{47,215} \mathrm{We}$ determined that levels of cytosolic $\mathrm{Ca}^{2+}$ concentration in the platelets remained low during a 24-h incubation, i.e. changing from $26 \pm 0.8$ to only $29 \pm 4 \mathrm{nM}$. Subsequently, we measured intracellular calpain activity before and after incubation of the platelets, using a newly developed procedure. Therefore, (incubated) platelets were loaded with the specific calpain substrate, tBoc-LM$\mathrm{CMC}$, and fluorescence development due to intracellular substrate cleavage was followed by stopped-flow fluorometry in the presence of EGTA to prevent contribution of possibly leaked calpain. ${ }^{214}$ In agreement with earlier results, ${ }^{214}$ the $\mathrm{Ca}^{2+}$-mobilizing agent, thapsigargin, caused an increase in basal calpain activity (figure $3 \mathrm{~A}$ ). In contrast, platelet incubation for $24 \mathrm{~h}$ resulted in a $33 \%$ decrease in calpain activity. As required, pre-treatment of the platelets with the specific calpain inhibitor, calpeptin, abolished fluorescence development.

A

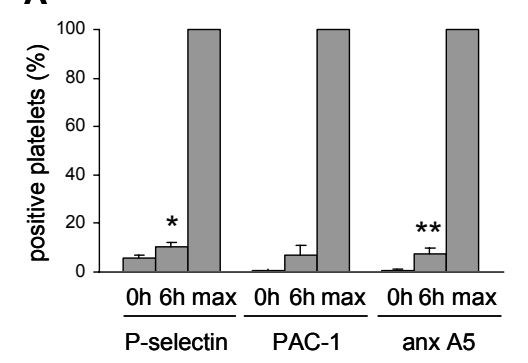

B

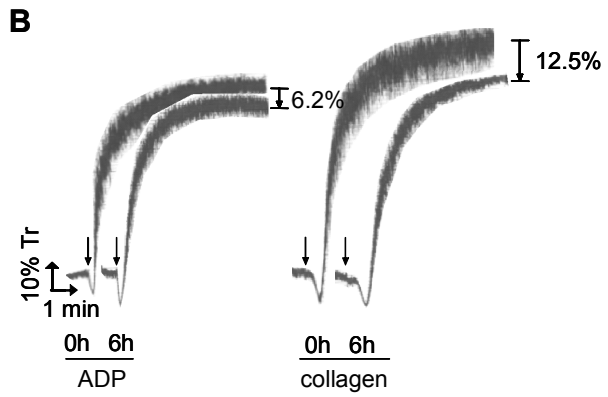

Figure 2. Minimal activation state of MPforming platelets. (A) Flow cytometric detection of activation markers on platelets, incubated in plasma for $6 \mathrm{~h}$. Platelets expressing P-selectin, activated integrin allbß3 (PAC1) or PS (Anx A5) after incubation in plasma for 0-6 h. Platelets were maximally activated (max), as described for $\begin{array}{lll}\text { figure } & 1 . & \text { (B) }\end{array}$ Representative aggregation traces of PRP stimulated with ADP $(10 \mu \mathrm{M})$ or collagen (2.5 $\mu \mathrm{g} / \mathrm{mL})$ after $6 \mathrm{~h}$ incubation. Data are means \pm S.E.M. $(n=3)$. ${ }^{*} p<0.1$ and ${ }^{* *} p<0.05$ compared to $0 \mathrm{~h}$. 
To determine whether the low calpain activity of resting platelets contributed to MP formation, PRP was incubated with calpeptin, and then evaluated on presence of coagulant MP. Calpeptin addition led to only a small, $35 \%$ reduction in the MP activity (figure 3B), indicating that the contribution of calpain-mediated protein cleavage in MP shedding is restricted.

Plasma contains ADP-degrading proteins that antagonize the effects of autocrine released ADP. ${ }^{116}$ Yet, to determine the possible involvement of autocrine mediators, PRP was rotated in the presence of a cocktail of inhibitors

A

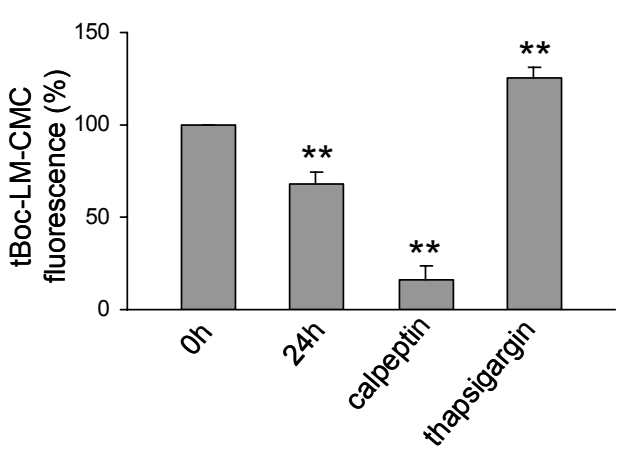

B
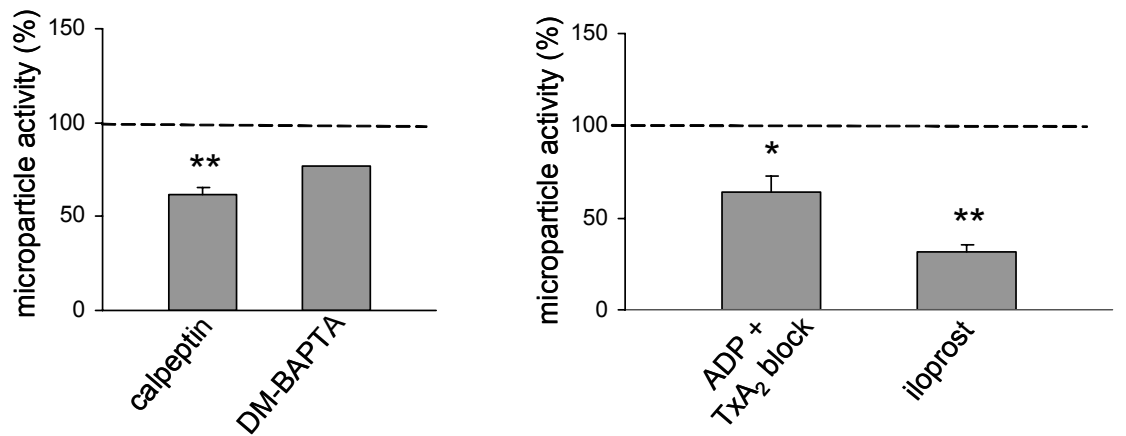

Figure 3. Involvement of calpain and autocrine mediators in shedding of coagulant MP. (A) Calpain activity measurement. PRP, incubated for $0-24 \mathrm{~h}$ was loaded with tBoc-LM-CMC, and intracellular calpain activity was assessed in the presence of 1 mM EGTA by stopped-flow fluorometry. Samples were pre-treated with calpain inhibitor, calpeptin $(140 \mu \mathrm{M})$, or activated with thapsigargin $(0.2 \mu \mathrm{M})$, as indicated. Data are expressed as percentages of $0-\mathrm{h}$ control. (B) MP coagulant activity. PRP was incubated for $6 \mathrm{~h}$ with vehicle or calpeptin $(140 \mu \mathrm{M})$; alternatively, with iloprost $(10 \mu \mathrm{M})$ or a mixture of MRS2179 $(20 \mu \mathrm{M})$, AR-C66931MX $(30 \mu \mathrm{M})$, apyrase $(1 \mathrm{U} / \mathrm{mL})$ and aspirin $(100 \mu \mathrm{M})$ to block $\mathrm{ADP}$ and thromboxane $\mathrm{A}_{2}$ effects. Platelets were pre-loaded with DMBAPTA1, where indicated. Data are expressed as percentages of control (thrombin peak levels, figure 1A). Means \pm S.E.M. $\left(n=5,{ }^{*} p<0.1\right.$ and ${ }^{* *} p<0.05$ vs. control). 
of the ADP and thromboxane $\mathrm{A}_{2}$ pathways. ${ }^{88}$ This treatment had a small, about $30 \%$ reducing effect on the formation of coagulant MP (figure $3 \mathrm{~B}$ ). Treatment of PRP with the cAMP-elevating compound, iloprost, at concentrations giving a 3-4 fold increase in cytosolic cAMP, resulted in an about $65 \%$ suppression of MP formation (figure $3 \mathrm{~B}$ ). Control experiments further showed that this MP formation was not due to incomplete anti-coagulation, because the level of thrombin-anti-thrombin complexes remained far below threshold levels of coagulation initiation ( $<3 \mathrm{ng} / \mathrm{mL}$ ) after $6 \mathrm{~h}$ or even 5 days of incubation of the PRP.

To study the involvement of allb $\beta 3$, the PRP was incubated with various clinically used blockers of this integrin: the high-affinity chimeric antibody, abciximab, a KGD-based peptide, eptifibatide, and the non-peptide antagonists, tirofiban and lotrafiban. ${ }^{216}$ These antagonists potently decreased MP formation in 6-h incubated PRP with $65-80 \%$, as determined from their coagulant activity (figure $4 \mathrm{~A}$ ). Also flow cytometry indicated that tirofiban reduced the number of MP in plasma with $87 \pm 5 \%(n=7)$. Further experiments showed that tirofiban inhibited thrombin generation, only when added to PRP before the 6-h incubation time (figure 4B). In contrast, tirofiban was without effect on coagulation when post-added to MP-containing PRP (figure 4C) or to PFP (not shown). These findings suggest that the anti-coagulant effect of integrin blockers is through inhibition of MP formation, and not by directly interfering with the thrombin generation process.

\section{Involvement of allb $\beta 3$-mediated actin cytoskeleton turnover and outside- in signaling}

As the actin cytoskeleton is of key importance for platelet structural integrity, we examined how compounds interfering with actin filament turnover influenced the shedding of MP. Platelets in plasma were incubated with cytochalasin D, an agent that caps actin filaments and prevents their polymerization. ${ }^{217}$ Remarkably, this resulted in a potent, two-fold increased formation of procoagulant MP (figure 5A). 

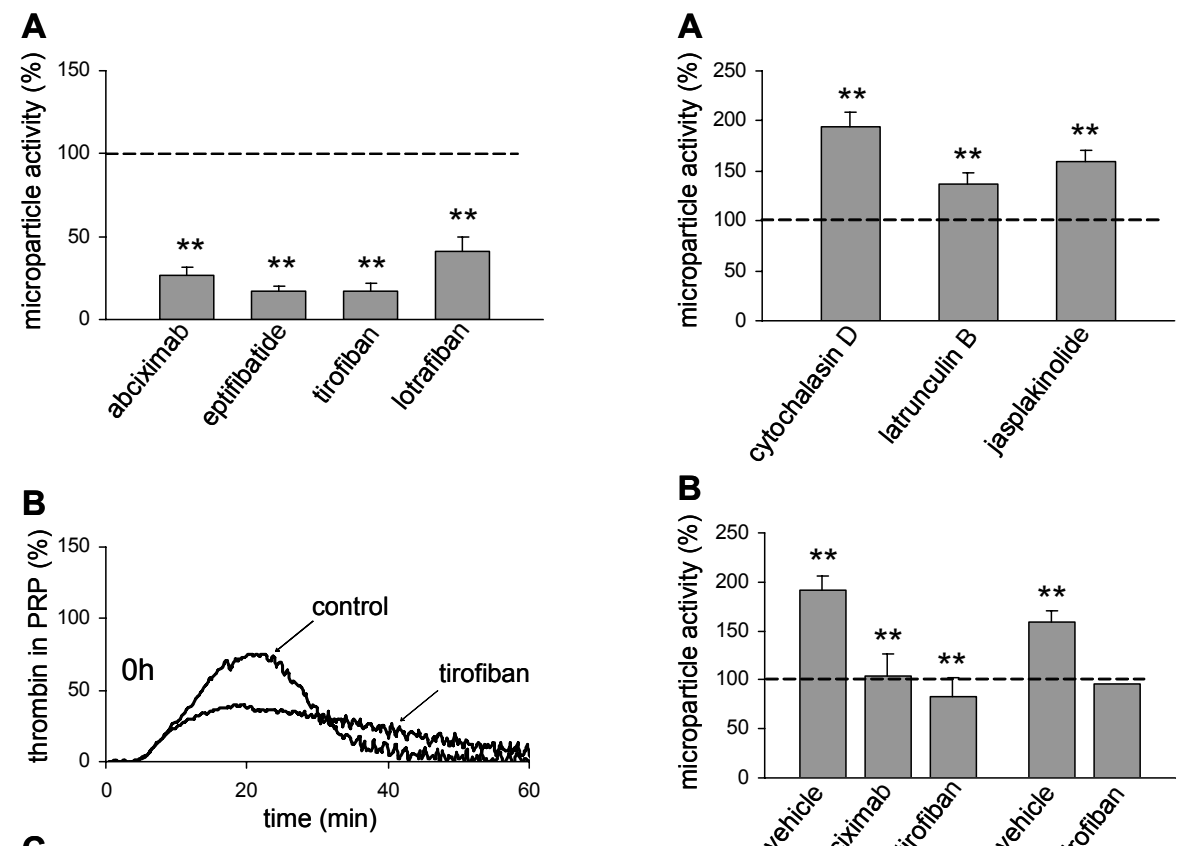

B
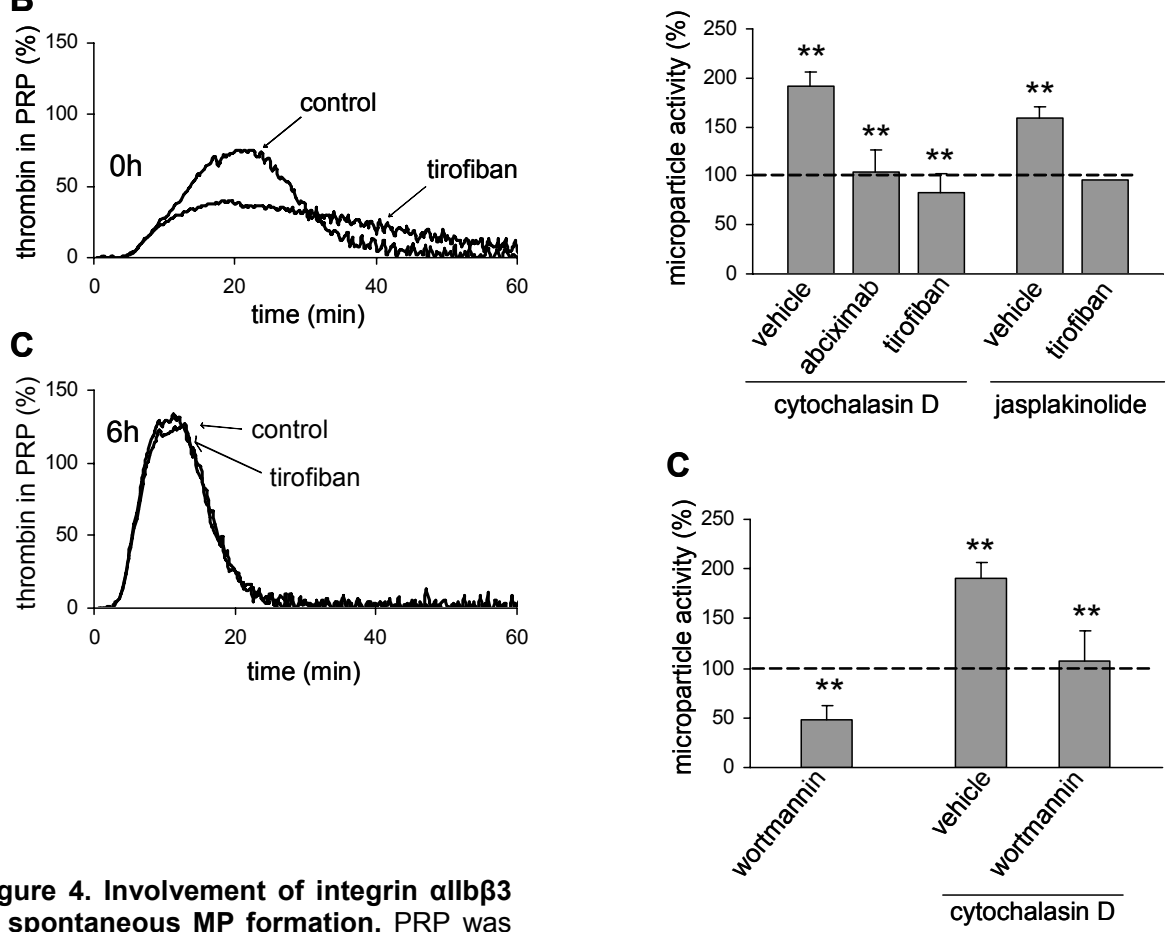

Figure 4. Involvement of integrin allb $\beta 3$ in spontaneous MP formation. PRP was incubated for $6 \mathrm{~h}$ with or without allbB3 inhibitors, abciximab $(20 \mu \mathrm{g} / \mathrm{mL})$, tirofiban $(2 \mu \mathrm{M})$, lotrafiban $(20 \mu \mathrm{M})$ or eptifibatide $(6$ $\mu M)$. (A) Coagulant activity of MP in plasma (PFP) after $6 \mathrm{~h}$ of incubation. (B) Effect of tirofiban added to fresh PRP. (C) Effect of tirofiban added after incubation of PRP for $6 \mathrm{~h}$. Shown are representative thrombin generation curves. Data are expressed as percentages of control PRP (6-h incubation without inhibitors), and are means \pm S.E.M. $\left(n=4,{ }^{* *} p<0.05\right)$.

Figure 5. Involvement of actin cytoskeleton in MP formation. (A) PRP was incubated for 6 $h$ with/without cytochalasin D $(10 \mu M)$, latrunculin $\mathrm{B}(0.5 \mu \mathrm{M})$ or jasplakinolide $(10 \mu \mathrm{M})$. (B) PRP was incubated for $6 \mathrm{~h}$ with cytochalasin $D$ in the presence of integrin blockers (figure 4). (C) PRP was incubated with cytochalasin D plus wortmannin $(1 \mu \mathrm{M})$. Data indicate MP coagulant activity as percentages of control PRP (incubation without drugs). ${ }^{* *} p<0.05$ vs. control PRP, ${ }^{* \star} p<0.05$ vs. vehicle $(n=3-4)$. 
A similar, stimulating effect was obtained, when platelets were incubated with latrunculin $B$, which inhibits filament polymerization by binding to actin monomers; or with jasplakinolide, which freezes platelet actin filaments but does allow severing. ${ }^{218}$ All compounds rearranged actin to a layer close to the plasma membrane, as was confirmed by staining of the platelets with fluorescent phalloidin.

Interestingly, the enhancing effects of cytochalasin $D$ and jasplakinolide on MP shedding were reversed by integrin blockade with abciximab or tirofiban (figure $5 \mathrm{~B}$ ). This led to the suggestion that $\alpha$ llb $\beta 3$ activity causes MP formation via destabilization of the actin cytoskeleton. The involvement of Src kinase and phosphoinositide 3-kinase was then considered, which protein kinases have been implicated in signaling of $\alpha$ llb $\beta 3$ to cytoskeletal changes. ${ }^{45}$ Incubation of PRP with the 3-kinase inhibitor wortmannin halved MP formation, and also reversed the enhancing effect of cytochalasin $D$ (figure $5 C$ ). Inhibition of Src kinases with $20 \mu \mathrm{M}$ PP1 or PP2 (but not the control substance PP3) reduced microvesiculation by $39 \pm 7 \%(n=5, p<0.01)$.

The cytochalasin D-enhanced and tirofiban-suppressed MP formation did not involve calpain activity, as calpeptin $(140 \mu \mathrm{M})$ treatment was without influence on this process. Furthermore, platelet treatment with cytochalasin $\mathrm{D}$ or tirofiban changed the intracellular calpain activity insignificantly with $4 \%$ and $7 \pm 3 \%$.

Although the allb $\beta 3$ activation state of platelets in plasma in general is low (compare figure $2 \mathrm{~A}$ ), the above results suggest that a low, residual integrin activity is sufficient to cause MP formation. Support for this came from flowcytometric measurements, showing that PAC1 binding to platelets stored in plasma was slightly above basal; the mean PAC1 binding reduced with 3 channel numbers by treatment of the platelets with cAMP-elevating iloprost.

The spreading of platelets over a fibrinogen surface is an event controlled by integrin outside-in signaling. ${ }^{219}$ We investigated whether this signaling during spreading also leads to MP formation. High-resolution, phase-contrast microscopy showed that many platelets, in a late stage of spreading, often developed circular arrays of cell-attached MP after forming lamellipods (figure $6 \mathrm{~A}$, see also movie A). Confocal fluorescence microscopy indicated that these MP 
A phase contrast ( $\mathrm{PHC})$

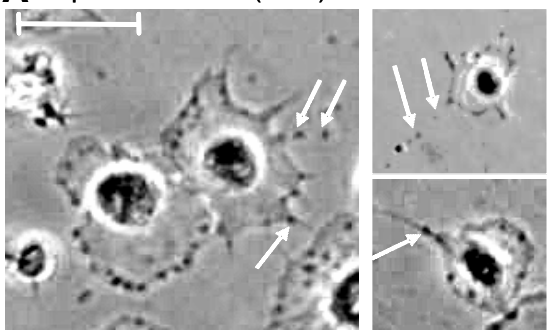

B phalloidin

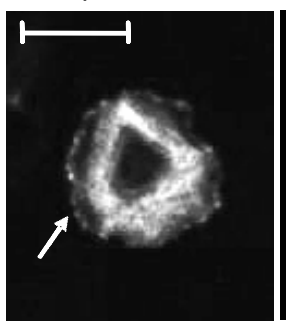

annexin A5

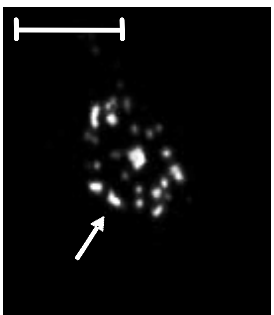

C PHC annexin A5 PAC1 merge

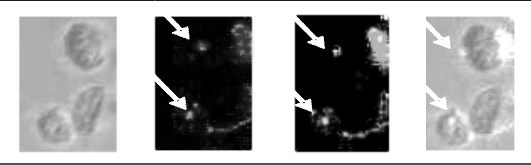

Figure 6. MP formation of spreading platelets. Platelets in Hepes buffer were immobilized on a fibrinogen surface for $45 \mathrm{~min}$ at room temperature. (A) Representative phase-contrast image of spreading platelets with MP (arrows). (B) Microscopic images of actin staining with AFphalloidin, and of PS exposure with OG-annexin A5. (C) Dual confocal staining of MP-forming platelets with $\mathrm{AF}$-annexin $\mathrm{A} 5$ and FITC-anti $\beta 3$. Bars represent $5 \mu \mathrm{m}$.

express PS and form at sites of disintegrated actin filaments (figure 6B). Dual labeling demonstrated PAC1 staining of the PS-positive MP (figure $6 \mathrm{C}$ ). Control experiments confirmed that integrin blockers antagonized both the spreading and MP formation.

Finally, thrombin generation experiments were performed with PRP from thrombocytopenic patients, who were transfused with MP-containing platelet preparations stored at blood bank conditions. After transfusion, the thrombin peak level in both PRP and PFP was notably increased, pointing to the presence of circulating MP in the patient blood (figure 7). Thus, MP produced during platelet storage actively contribute to the coagulation process, when transfused into patients. 

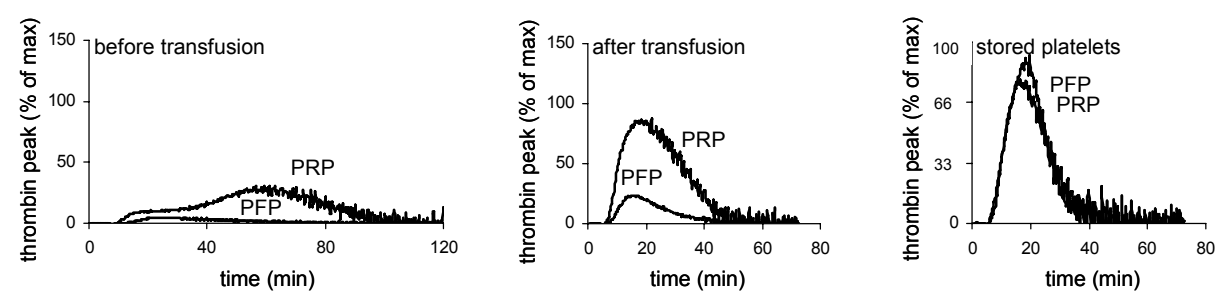

Figure 7. Thrombin generation of PRP and PFP from a thrombocytopenic patient before and after transfusion with a platelet-plasma preparation. Platelet counts in patient PRP before and after transfusion were 16 and $64 \times 10^{9} / \mathrm{L}$, respectively. PRP and PFP samples pre- and postperfusion were triggered with tissue factor, as described for figure $1 \mathrm{~A}$. Also shown is thrombin generation of the PRP and plasma that was used for transfusion. Increased thrombin generation in post-transfusion PFP points to activity of coagulant MP. Representative thrombin generation curves are given (triplicate assays).

\section{Discussion}

This report describes the novel finding that human platelets during storage form procoagulant MP as a 'spontaneous' reaction, in a way dependent on integrin allb $\beta 3$ and cytoskeleton turnover, but at minimal platelet activation and calpain activity. This process is of clinical relevance, since MP-producing platelet preparations are routinely transfused to patients, and can enhance coagulation.

Flow cytometry was used to detect the gradual accumulation of MP in stored platelets. Complementary to this, thrombin generation measurements allowed quantification of the amounts of procoagulant MP in plasma. We find that the spontaneous MP formation is an ongoing process in platelets, which relies on integrin signaling even when platelet activation is minimal. Only a minority of the MP-producing platelets bound PAC1 mAb, or had signs of surface expression of P-selectin or PS. Furthermore, the aggregation tendency of MP-producing platelets was no more than slightly altered. The results do not indicate whether MP are produced by a small fraction of the platelets or by all platelets. In favour of the latter possibility is the overall decrease in mean platelet volume of about $10 \%$, which is detectable after prolonged incubation.

The mechanism of MP shedding from resting platelets, typically, differs from the shedding mechanism that is described for platelets treated with potent agonists like $\mathrm{Ca}^{2+}$-ionophore or collagen/thrombin. In these cases, it has been shown that basal calpain activity is insufficient for its protease activity to 
degrade cytoskeletal components, and that agonist-induced $\mathrm{Ca}^{2+}$ influx is required to reach the high, (sub)micromolar $\left[\mathrm{Ca}^{2+}\right]_{i}$ levels triggering this protease activity. Similarly, with these potent agonists, $\mathrm{Ca}^{2+}$ influx was required to cause $\mathrm{Ca}^{2+} /$ calpain-dependent MP formation. ${ }^{47,209,215,220}$ In contrast, in the present setting, we find that $\mathrm{Ca}^{2+}$ levels remain low in the stored platelets (with low extracellular $\mathrm{Ca}^{2+}$ in the storage medium), while the basal calpain activity even decreases. Furthermore, we find that $\mathrm{Ca}^{2+}$ chelation and calpain inhibition have only minor effects on MP formation. Together, this indicates that the $\mathrm{Ca}^{2+} /$ calpain pathway plays only a minor role in MP shedding during platelet storage.

We find that integrin allb $\beta 3$ plays an important role in the shedding process of resting platelets. Some authors have reported that $\alpha$ llb $\beta 3$ activation and aggregation are also involved in MP production by platelets stimulated with potent agonists ${ }^{209,221}$, while others have not found such involvement of the integrin. ${ }^{222,223}$ In activated platelets, allbß3 can furthermore regulate calpain activation. ${ }^{224}$ Based on these and other findings, Fox ${ }^{47}$ and Wiedmer ${ }^{220}$ and colleagues have suggested that there may be more than one mechanism of MP formation. Taken together with the present results, we conclude that there must be a separation between the $\mathrm{Ca}^{2+} /$ calpain and the $\alpha$ llb $\beta 3$ pathways, although both may operate alongside each other or synergize. Mostly the allb $\beta 3$ mediated, calpain-independent pathway is then responsible for MP formation by stored platelets.

There is recent evidence to indicate that integrins on a cell surface are in constitutive equilibrium between inactive and active conformations. ${ }^{225}$ This raises the possibility that patches of active (signaling) integrins on otherwise unstimulated platelets can trigger local shedding of MP. This would also explain why inhibition of Src or phosphoinositide 3-kinase signaling suppresses MP formation, likely via interference in outside-in signaling. However, it should be noted that, in stimulated platelets, these protein kinases also contribute to inside-out signaling. ${ }^{226}$

A remarkable finding is that the allb $\beta 3$-mediated MP formation increases with compounds like cytochalasin $\mathrm{D}$, interfering with the actin cytoskeleton 
turnover. It is known that actin-binding proteins, such as filamin and cofilin, provide physical interaction of the cytoskeleton with the $\beta 3$ cytoplasmic tail of integrin allb $\beta 3 .{ }^{217}$ Particularly, the phosphoinositide 3-kinase signaling pathway links between allb $\beta 3$ and actin dynamics. ${ }^{45}$ This leads to the idea that integrin signaling via this pathway locally destabilizes the cytoskeleton (via cofilin), with as a result disconnection of the plasma membrane and shedding of MP. Support for such a mechanism comes from the hitherto unrecognized detection of MP at the edges of platelets spread on fibrinogen and the observed effect of Src kinase inhibitors. Thus, activated integrins may act by suppression of the (spontaneous) rearrangement of the actin cytoskeleton and, conversely, cytoskeleton turnover suppresses integrin activation and hence MP formation.

Integrin blockers have a clinically relevant, anti-coagulant effect in the circulation by suppressing the availability of procoagulant lipid surface. ${ }^{227}$ The present findings indicate that this anti-integrin effect particularly relies on inhibition of MP formation. We can thus conclude that integrin-mediated shedding of MP is a uniquely regulated cellular response that contributes to coagulant activity in a clinically relevant way.

Acknowledgments: We thank dr. E.C.M. van Pampus for providing plasma from a thrombocytopenic patient.

Reprinted with permission of Federation of the European Biochemical Societies from 'Shedding of microparticles from unstimulated platelets by integrinmediated destabilization of actin cytoskeleton', by S. Cauwenberghs FEBS Letters, 580, 5313-20, 2006. 



\title{
Chapter 6
}

\section{Effect of storage on agonist-induced calcium responses in platelets}

\author{
a pilot study \\ Sandra Cauwenberghs, Joyce Curvers, \\ Marion A.H. Feijge and Johan W.M. Heemskerk
}

(to be submitted) 


\section{Abstract}

Platelets stored for transfusion purposes gradually loose their functionality and viability in a process known as platelet storage lesion. Considering that an increase in cytosolic $\left[\mathrm{Ca}^{2+}\right]_{i}$ is one of the first common signaling steps induced by platelet agonists, we investigated the effect of storage on $\mathrm{Ca}^{2+}$ signal generation in platelets that were stored at blood bank conditions for up to 7 days. Storage resulted in lower $\mathrm{Ca}^{2+}$ responses after platelet stimulation via either G-protein coupled receptors (ADP, thromboxane, thrombin) or tyrosine kinase-linked receptors (collagen). The lower $\mathrm{Ca}^{2+}$ responses were accompanied by a reduction in agonist-induced P-selectin expression. Flow cytometry revealed that part of the stored platelets had a delayed $\mathrm{Ca}^{2+}$ response after collagen receptor activation. Furthermore, when perfused in whole blood over a collagen surface at moderately high, arterial shear rates, prolonged platelet storage resulted in lower and sometimes more delayed $\mathrm{Ca}^{2+}$ signals. Taken together, these results show that the reduced $\mathrm{Ca}^{2+}$ signal generation in stored platelets is detectable under static and flow conditions, and involves a mechanism that is independent of the signaling pathway and type of agonist. 


\section{Introduction}

Mobilization of cytosolic $\mathrm{Ca}^{2+}$ from intracellular stores is a key signaling step in many physiological platelet reactions, such as shape change, secretion, integrin activation, aggregation and procoagulant activity. ${ }^{228,229}$ Accordingly, $\mathrm{Ca}^{2+}$ mobilization is necessary for platelets to form intravascular thrombi and to initiate coagulation after vessel wall damage. ${ }^{12,51,230}$ Most platelet agonists are capable of raising $\left[\mathrm{Ca}^{2+}\right]_{i}$ via stimulation of a phosphoinositide 4,5-biphosphate specific phospholipase $C(P L C)$. These include agonists activating the $G_{q^{-}}$ protein coupled receptors for ADP $\left(P 2 Y_{1}\right)$, thromboxane $A_{2}(T P \alpha)$, and thrombin (PAR1/4), all of which stimulate PLC $\beta$ isoforms. ${ }^{229,231}$ In addition, the collagen receptor glycoprotein VI (GPVI) stimulates, via a protein tyrosine kinase pathway, the different PLCY2 isoform, which also signals to increased $\left[\mathrm{Ca}^{2+}\right]_{i} \cdot{ }^{92}$

During storage at blood bank conditions, platelets undergo a number of structural and functional changes, which can be classified as either an increase in surface activation markers or a decrease in responsiveness. ${ }^{232}$ One of these changes concerns a decrease in $\mathrm{Ca}^{2+}$ responses. ${ }^{100,108}$ Here, we investigated whether this decreased responsiveness results from specific alterations caused by PLC $\beta$ - or PLCY2-stimulating agonists.

\section{Materials and methods}

\section{Materials}

Fura-2, Fluo-3 and Fluo-4 acetoxymethyl esters as well as pluronic were from Molecular Probes (Leiden, the Netherlands); Horm-type fibrillar I collagen was obtained from Nycomed (Munich, Germany); fluorescein isothiocyanate (FITC)-labeled anti-CD62P monoclonal antibody (mAb) was from CLB (Amsterdam, the Netherlands); D-Phe-Pro-Arg chloromethyl ketone (PPACK) from Calbiochem (San Diego, CA, USA). Convulxin was purified from crude snake venom, as described. ${ }^{213}$ Proteinase-activated receptor-1 (PAR1) peptide SFLLRN was purchased from Bachem (Bubendorf, Switzerland); human $\alpha$ thrombin was from Kordia Life Sciences (Leiden, the Netherlands). Other reagents were from Sigma (St. Louis, MO, USA). 


\section{Collection of blood and platelet preparation}

Whole blood was drawn from healthy volunteers, who gave full informed consent. The blood was collected in 1/10 volume citrate medium (CPD, $1.9 \mathrm{mM}$ citric acid, $11.0 \mathrm{mM}$ trisodium citrate, $2 \mathrm{mM} \mathrm{NaH}_{2} \mathrm{PO}_{4}$ and $15.8 \mathrm{mM}$ glucose, $\mathrm{pH}$ 7.0), and centrifuged at $240 \mathrm{~g}$ for 15 minutes. Platelet-rich plasma (PRP) from freshly donated blood was carefully removed, leaving the buffy coat untouched. For platelets stored under standard blood bank conditions, whole blood was processed to buffy coats and filtrated before storage to remove leukocytes, as described before. ${ }^{100}$ In the latter case, PRP, containing $1 \times 10^{12}$ platelets/L and $<10^{6}$ leukocytes/L, was kept at $22^{\circ} \mathrm{C}$ in a flatbed shaker.

To prepare washed platelets, fresh or stored PRP was mixed with $1 / 6$ volume ACD solution ( $80 \mathrm{mM}$ trisodium citrate, $52 \mathrm{mM}$ citric acid and $180 \mathrm{mM}$ glucose), supplemented with $0.1 \mathrm{unit} / \mathrm{mL}$ apyrase, and then centrifuged at $800 \mathrm{x}$ $g$ for 15 minutes. Platelets were washed, and resuspended in Hepes buffer $\mathrm{pH}$ 7.45 (136 mM NaCl, $2.7 \mathrm{mM} \mathrm{KCl}, 10 \mathrm{mM}$ Hepes, $2 \mathrm{mM} \mathrm{MgCl}$, $2 \mathrm{mM}$ glucose and $1 \mathrm{mg} / \mathrm{mL}$ bovine serum albumin). ${ }^{158}$ Cells were counted using a Beckman Coulter counter (Miami, FL, USA).

\section{Measurements of $\left[\mathrm{Ca}^{2+}\right]_{i}$ in platelet suspensions}

For spectrofluorometry, platelets in PRP were loaded with Fura-2 acetoxymethyl ester, as described. ${ }^{158}$ After a wash step, the platelets were resuspended in Hepes buffer $\mathrm{pH} 7.45$ (no $\mathrm{CaCl}_{2}$ was added). In stirred suspensions of Fura-2-loaded platelets, changes in fluorescence were monitored by ratio fluorometry, and then converted to nanomolar $\mathrm{Ca}^{2+}$ concentrations following a standard calibration procedure. ${ }^{158}$ Fluorescence was recorded at $37^{\circ} \mathrm{C}$ with a SLM-Aminco spectrofluorometer (Rochester, NY, USA).

\section{Flow cytometry}

Platelets $\left(250 \times 10^{9} / \mathrm{L}\right)$ were loaded with Fluo-4 $(3 \mu \mathrm{M})$ acetoxymethyl ester in the presence of pluronic $(1 \mathrm{mg} / \mathrm{mL})$. Here, flow cytometry was used to measure fluorescence changes in individual platelets. Increases in Fluo-4 fluorescence were measured before and after activation with convulxin (25 
$\mathrm{ng} / \mathrm{mL}$ ) for exactly 10-30 seconds. P-selectin, a measure of platelet secretion, was measured after 10 minutes incubation with a specific FITC-labeled mAb $(\alpha$ CD62P-FITC $) .^{100}$

\section{Whole blood flow experiments}

Before start of a flow experiment, reconstituted, freshly isolated, anticoagulated blood was recalcified with $16.6 \mathrm{mM} \mathrm{CaCl}_{2}$ (giving physiological, free $\mathrm{Ca}^{2+}$ and $\mathrm{Mg}^{2+}$ concentrations of about $2 \mathrm{mM}$ ) in the presence of $20 \mu \mathrm{M}$ (f.c.) PPACK to avoid coagulation. The blood was supplemented with $25 \%$ of Fluo-3loaded $(5 \mu \mathrm{M})$ platelets from the same donor (either freshly isolated or 7 days old). Coverslips were coated with Horm-type I collagen, and mounted onto a transparent, parallel-plate flow chamber that was placed on the stage of a fluorescence microscope. ${ }^{90}$ Blood was perfused for 4 minutes at a continuous wall-shear rate of 1000/s. ${ }^{91}$ After perfusion, high-resolution phase-contrast images were recorded in real-time with an intensified, charge-coupled device camera and a Visitech digital imaging system (Sunderland, UK). Calcium responses were measured during perfusion of whole blood samples supplemented with autologous, Fluo-3-labeled platelets (25\% labeled platelets). Changes in Fluo-3 fluorescence were converted into nanomolar increases in $\left[\mathrm{Ca}^{2+}\right]_{\mathrm{i}}$ by a pseudo-ratioing procedure. ${ }^{233}$ Images were taken from representative microscopic fields and analyzed with Quanticell software (Visitech). Fractions of stably adherent and responding platelets with increased fluorescence were counted, as described. ${ }^{9}$

\section{Results}

\section{Decreased agonist-induced $\mathrm{Ca}^{2+}$ responses in stored platelets}

Agonist-induced $\mathrm{Ca}^{2+}$ responses were determined in platelets, after storage for 2,4 or 7 days under blood bank conditions. The cells were loaded with the $\mathrm{Ca}^{2+}$ probe Fura-2, pre-incubated with apyrase to prevent ADP receptor desensitization, and stimulated with agents that were applied at submaximal concentrations. The maximal increases in $\left[\mathrm{Ca}^{2+}\right]_{i}$ diminished with increasing storage time, regardless of whether the platelets were stimulated with the 
P2Y $1 / P 2 Y_{12}$ agonist, ADP, the TP $\alpha$ agonist U46619 or the PAR1 agonist, SFLLRN (figure $1 \mathrm{~A}$ ). This suggested gradual impairment of a common signaling pathway, since all these agonists mobilize cytosolic $\mathrm{Ca}^{2+}$ via the $\mathrm{G}_{\mathrm{q}}$ and PLC $\beta$ signaling route. In addition, maximal increases in $\left[\mathrm{Ca}^{2+}\right]_{i}$ did also reduce with time of storage, when the platelets were stimulated with convulxin (figure $1 \mathrm{~A}$ ), i.e. a GPVI agonist signaling via a different route involving protein tyrosine kinases and PLCY2.

A
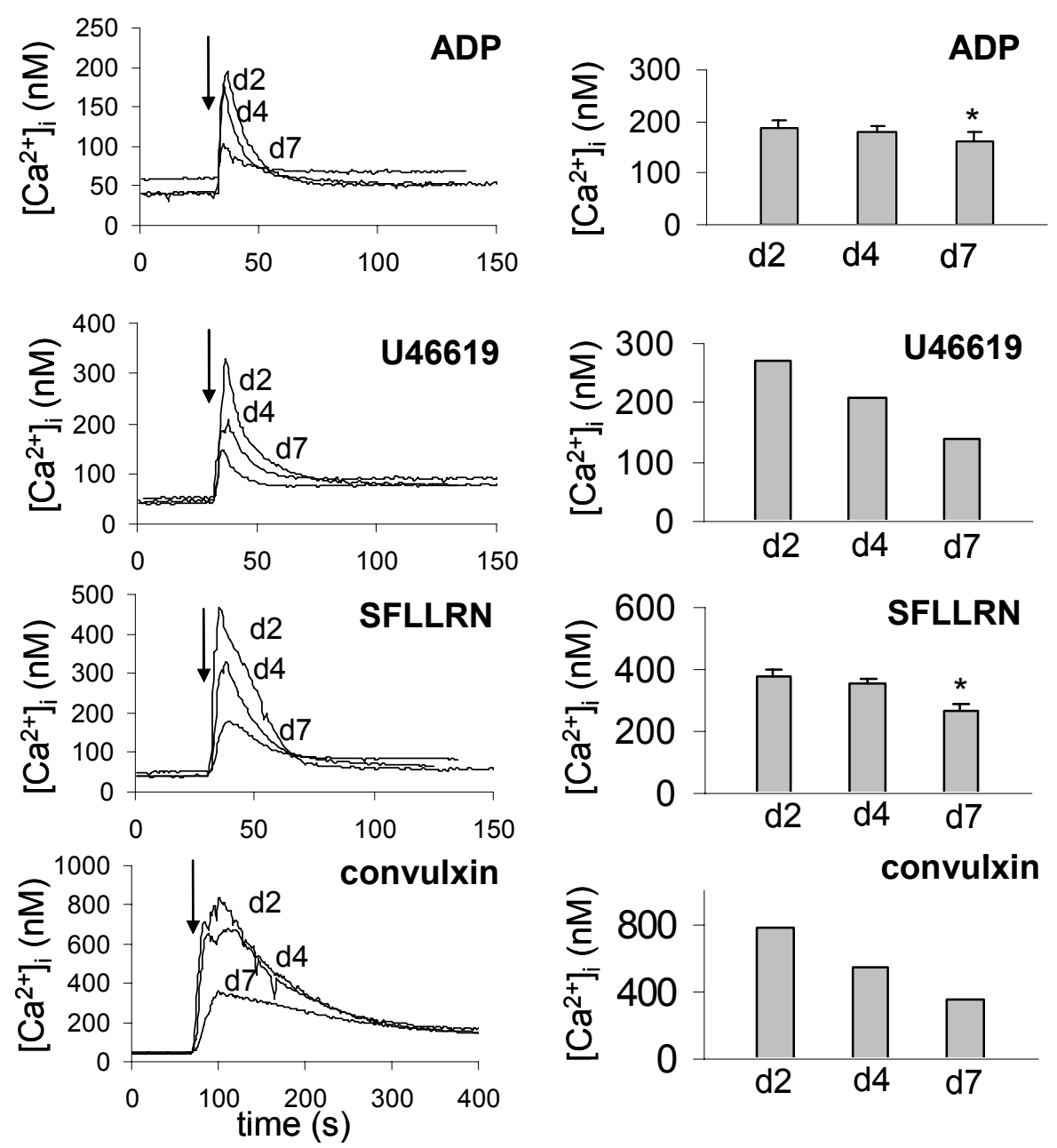


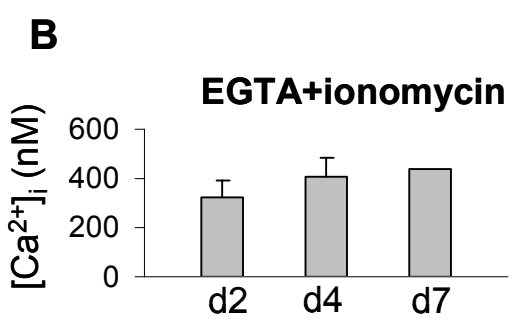

Figure 1. Decreased agonist-induced $\mathrm{Ca}^{2+}$ responses after storage of platelets. Changes in $\left[\mathrm{Ca}^{2+}\right]_{\text {i }}$ were measured by ratio fluorometry in washed, Fura-2-loaded platelets, which were previously stored for 2,4 or 7 days at blood bank conditions. (A) Platelets in Hepes buffer, containing $1 \mathrm{mM} \mathrm{CaCl}$, were activated with ADP $(20 \mu \mathrm{M})$, SFLLRN $(15 \mu \mathrm{M}), \mathrm{U} 46619(2.5 \mu \mathrm{M})$ or convulxin $(25 \mathrm{ng} / \mathrm{mL})$, as indicated (arrows). (B) Fura-2-loaded platelets in Hepes buffer containing EGTA $(0.1 \mathrm{mM})$ were activated with ionomycin $(10 \mu \mathrm{M})$. Representative traces are shown; data are mean values \pm SEM $(n=2-4) ;{ }^{*} p<0.05$ compared to 2 days.

That stored platelets gradually lost their responsiveness was confirmed by measurement of agonist-induced surface expression of P-selectin, which is known to be a $\mathrm{Ca}^{2+}$-dependent platelet response. For instance, after stimulation with SFLLRN $(15 \mu \mathrm{M})$, the fraction of platelets expressing P-selectin decreased from $73.2 \pm 3.6 \%$ to $39.6 \pm 4.8 \%$ to $30 \pm 7.1 \%$ and $31.8 \pm 3.2 \%$ after storage for $0,2,4$ and 7 days, respectively (mean \pm SEM, $n=4$ ) (data not shown).

We hypothesized that the impaired $\mathrm{Ca}^{2+}$ responses of stored platelets were due to a gradual depletion of the intracellular $\mathrm{Ca}^{2+}$ stores, which are located in the endoplasmic reticulum. To investigate this, Fura-2-loaded platelets were treated with the $\mathrm{Ca}^{2+}$ ionophore, ionomycin, in the presence of EGTA, i.e. a condition which causes full depletion of the intracellular stores, while eliminating influx of extracellular $\mathrm{Ca}^{2+}$. However, as indicated in figure $1 \mathrm{~B}$, ionomycininduced increases in $\left[\mathrm{Ca}^{2+}\right]_{i}$ remained unchanged in platelets that were stored for up to 7 days. On the other hand, when the cells were activated with the PAR1/4 agonist, thrombin, in EGTA medium, this resulted in a $\mathrm{Ca}^{2+}$ response that diminished with $40 \pm 5 \%$ (mean $\pm \mathrm{SEM}, n=3$ ) after prolonged storage (data not shown). This indicated that thrombin-induced $\mathrm{Ca}^{2+}$ mobilization was impaired, while the $\mathrm{Ca}^{2+}$ store content was unchanged.

A typical observation, most clearly seen with the agonist convulxin, was that prolonged storage of platelets resulted in a reduction of the initial $\mathrm{Ca}^{2+}$ increase (figure $1 \mathrm{~A}$ ). To investigate this further, we used flow cytometry as a technique capable to identify differences between platelet populations. Thus, platelets stored for 2 or 7 days were loaded with the 488-nm excitable probe Fluo-4, and increases in fluorescence were measured - which correspond to increases in 
$\left[\mathrm{Ca}^{2+}\right]_{i}$ - after certain time points of activation. Controls with fresh platelets showed that stimulation with ionomycin provoked a marked increase in fluorescence in all platelets (figure 2A). Stimulation with convulxin during 30 seconds elevated the fraction of platelets with high fluorescence, i.e. with high $\left[\mathrm{Ca}^{2+}\right]_{\mathrm{i}}$. Typically, when this experiment was performed with platelets that were stored for 7 days, a smaller fraction of the cells was high in $\left[\mathrm{Ca}^{2+}\right]_{\mathrm{i}}$ at the same time points, e.g. at 20 seconds after activation (figure 2B). Apparently, the storage not only reduced the extent of agonist-evoked $\mathrm{Ca}^{2+}$ mobilization, but also diminished the rate of this process at least in a sub-population of the platelets.

A
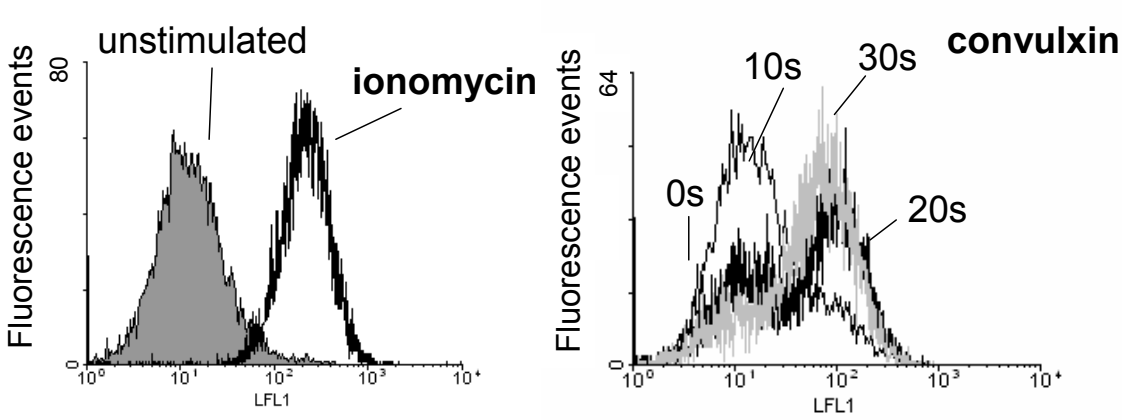

$\mathbf{B}$

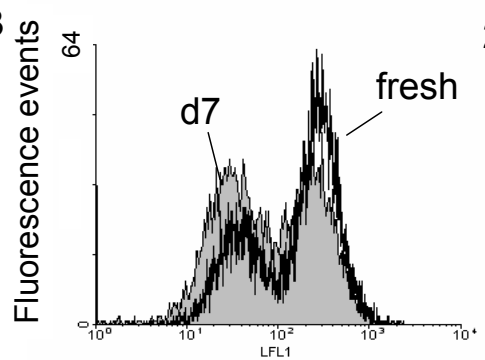

$20 s$

$20 s$
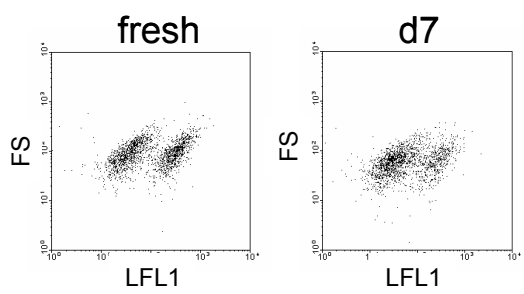

Figure 2. Increased delay in $\mathrm{Ca}^{2+}$ response after storage of platelets. Platelets from a single donor, freshly isolated or stored for 7 days at blood bank conditions, were loaded with Fluo-4, i.e. a $\mathrm{Ca}^{2+}$ probe relatively resistant to leakage out of the cells. (A) Fresh, Fluo-4-loaded platelets in Hepes buffer remained unstimulated (gray area) or were activated at $37^{\circ} \mathrm{C}$ with ionomycin $(10 \mu \mathrm{M}$, 60 seconds) or convulxin (25 ng/mL, 10-30 seconds) in the presence of $1 \mathrm{mM} \mathrm{CaCl}_{2}$ (no stirring to prevent aggregate formation). Samples were analyzed by flow cytometry before and after activation, as indicated. (B) Fresh or 7-day old Fluo-4-loaded platelets were activated with convulxin for exactly 20 seconds. Note higher fraction of fresh cells with increased fluorescence (increased $\left[\mathrm{Ca}^{2+}\right]_{i}$ ). Data are representative for 3 experiments. 


\section{Reduced $\mathrm{Ca}^{2+}$ responses under flow of stored platelets}

The affected response of stored platelets with the GPVI agonist convulxin led us to study the activation of the cells under more physiological conditions, i.e. flowing in whole blood over a collagen (GPVI-activating) surface. Therefore, fixed numbers of Fluo-3-loaded platelets (freshly isolated or 7-days old) were added to blood from the same donor, and changes in fluorescence were monitored by microscopic video imaging during high-shear perfusion, when individual platelets started to adhere to the collagen surface. Counting of the numbers of stably adherent platelets with detectable increases in $\left[\mathrm{Ca}^{2+}\right]_{i}$ gave lower values in case of stored platelets (figure $3 \mathrm{~A}$ ), which again suggested that these platelets were delayed in GPVI signaling. Furthermore, analysis of the changes in $\left[\mathrm{Ca}^{2+}\right]_{i}$ showed that the $\mathrm{Ca}^{2+}$ responses of individual platelets were lower, again, after storage of the cells (figure $3 \mathrm{~B}, \mathrm{C}$ ). Individual, stored platelets often showed a period of transient $\left[\mathrm{Ca}^{2+}\right]_{i}$ peaks, which gradually changed into a stage of prolonged elevation. Together, these results show that the delayed and reduced $\mathrm{Ca}^{2+}$ signaling of stored platelets is relevant for conditions of thrombus formation under flow.

\section{Discussion}

The present results point to a reduced $\mathrm{Ca}^{2+}$ responsiveness of stored platelets, which is not limited to certain agonists, but extends to agents activating PLC $\beta$ (via $G_{q}$ ) and PLCY2 (via tyrosine kinases). As far as studied, these reduced $\mathrm{Ca}^{2+}$ responses are accompanied by diminished secretion ( $P$ selectin expression). Single cell analysis, both using flow cytometry and fluorescence microscopic imaging, further indicated that part of the stored platelets are delayed in responsiveness to GPVI stimulation and, hence, more difficult to trigger. The reduced responsiveness does not appear to be due to depletion of the intracellular $\mathrm{Ca}^{2+}$ stores, but to a diminished agonist-induced mobilization of $\mathrm{Ca}^{2+}$ out of the stores. Furthermore, the lower $\mathrm{Ca}^{2+}$ signals are well detectable in single platelets during high-shear perfusion, under conditions of collagen-induced thrombus formation. 
A
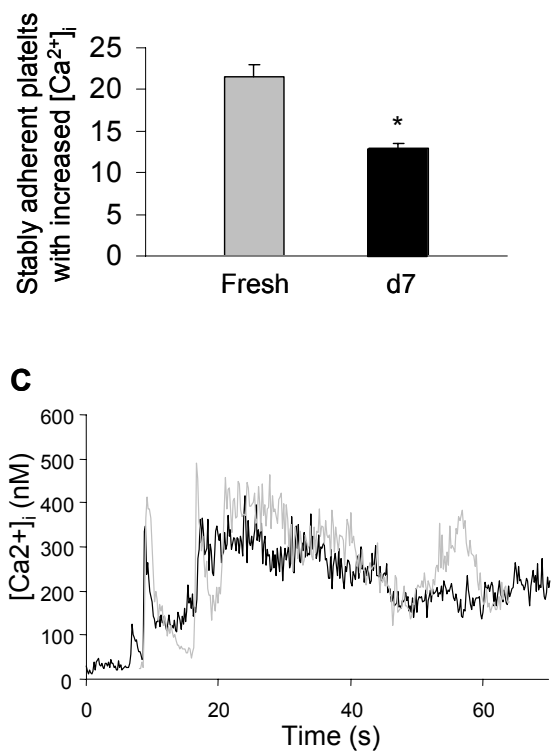

B
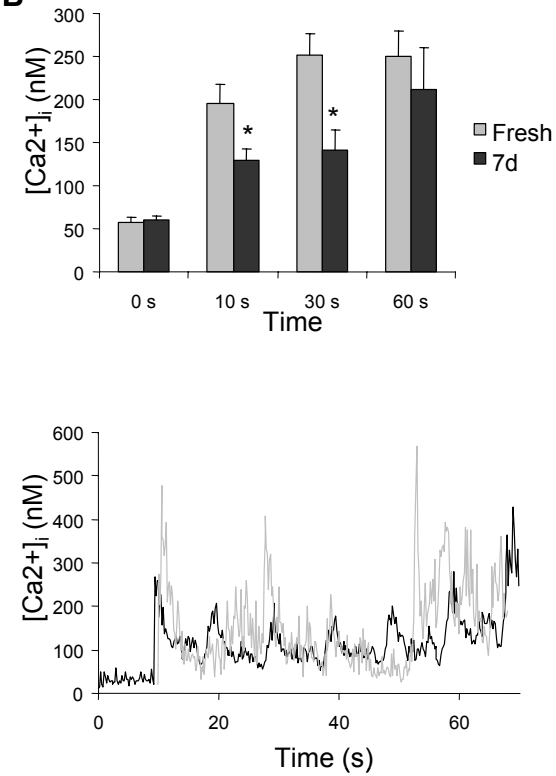

Figure 3. Decreased collagen-induced $\mathrm{Ca}^{2+}$ responses in flowing blood containing stored platelets. Donor blood was supplemented with autologous freshly isolated or 7-day stored platelets, which were pre-loaded with Fluo-3 (finally in blood, $25 \%$ of Fluo3-loaded platelets). Blood was then perfused over a collagen surface at 1000/s, and fluorescence changes in single, adhering platelets were measured in real-time during flow by camera video imaging. (A) Numbers of stably adherent platelets showing detectable increases in $\left[\mathrm{Ca}^{2+}\right]_{i}$ in a period of 20 seconds (100 images) per microscopic field $(100 \times 100 \mu \mathrm{m})($ mean \pm SEM, $n=3)$. (B) Averaged $\left[\mathrm{Ca}^{2+}\right]_{i}$ in single adherent platelets at $0,10,30$ and 60 seconds after adhesion to collagen (mean \pm SEM, $n=25$ cells). (C) Typical traces of changes in $\left[\mathrm{Ca}^{2+}\right]_{i}$ of some fresh and 7-day old platelets. Data are representative for 6 experiments with platelets from 2 different donors; ${ }^{*} p<0.05$ compared to fresh.

It is tempting to speculate on the mechanism responsible for this reduced $\mathrm{Ca}^{2+}$ mobilization in stored platelets. Earlier, it was shown that inositol 1,4,5trisphosphate-induced mobilization of $\mathrm{Ca}^{2+}$ in platelets is affected by the glutathione oxidation state of the cells. ${ }^{234}$ Other authors describe that storage increases glutathione oxidation in platelets, which was considered evidence for a lower resistance of stored platelets against metabolic stress. ${ }^{235,236}$ Accordingly, the lower $\mathrm{Ca}^{2+}$ responses might be due to partial oxidation of sulfhydryl groups of proteins like the inositol 1,4,5-trisphosphate receptor, involved in $\mathrm{Ca}^{2+}$ store depletion. Whether this indeed is the case, remains to be investigated. It is important to note that the 7-day stored platelets still showed spiking increases as well as rapid decreases in $\left[\mathrm{Ca}^{2+}\right]_{\text {, }}$, which strongly suggests that ATP-consuming $\mathrm{Ca}^{2+}$-ATPases, which are responsible for the falling parts of the spiking $\mathrm{Ca}^{2+}$ traces, are still normally active. This is in line with the 
observation that the mitochondrial membrane potential and, hence, the ATP energy state are not affected by storage for up to 7 days. ${ }^{237,238}$

Many investigations have indicated that adequate $\mathrm{Ca}^{2+}$ signaling is important for normal platelet function. This is apparent, for instance, from the mild bleeding tendency seen in subjects, whose platelets have a decreased level of membrane-associated $G_{\mathrm{q} \alpha}$ or display a selective deficiency in PLC $\beta 2 .{ }^{239,240}$ Consequently, for an effective hemostatic effect, it is important that platelets that are used for prophylactic transfusion purposes retain their normal $\mathrm{Ca}^{2+}$ responses. Although the current experiments have not yet revealed the mechanism responsible for the gradual impairment of platelet $\mathrm{Ca}^{2+}$ responses, they urge for further improvement of storage methods that preserve the high responsiveness of transfusion platelets. 



\title{
Chapter 7
}

\author{
Novel methodology \\ for assessment of prophylactic \\ platelet transfusion therapy \\ by measuring \\ increased thrombus formation \\ and thrombin generation
}

Sandra Cauwenberghs, Marion A.H. Feijge, Evi Theunissen, Johan W.M. Heemskerk, Elisabeth C.M. van Pampus*

and Joyce Curvers*

British Journal of Haemathology

2007; 136:480-90

Reprinted with permission 


\begin{abstract}
Currently, patients developing severe thrombocytopenia during chemotherapy treatment are prophylactically transfused with platelets. We developed two platelet function tests to report the improved hemostasis in the transfused patients, which were capable of detecting aberrant responsiveness of the platelets after transfusion. First, in a whole-blood flow test, platelet adhesion and thrombus formation were determined under high-shear flow conditions. Second, the procoagulant function of platelets was assayed in platelet-rich plasma by measurement of thrombin generation. Experimental conditions were established, where flow-induced adhesion and thrombin generation test parameters increased semi-linearly with the platelet concentration, and informed on the activation properties of platelets. The transfusion effects were evaluated for 38 thrombocytopenic patients, who were transfused with platelets stored in plasma or in synthetic medium (platelet additive solution II). In most but not all patients, transfusion resulted in increased adhesion and thrombus formation, as well as in improved plateletdependent coagulation. Taken together, the increase in platelet count after transfusion explained $57 \%$ of the overall improvement in platelet function. In acute graft-versus-host disease, thrombus formation was normal, while plateletdependent coagulation was higher than expected. We conclude that assessment of flow-induced adhesion and thrombin generation in acquired thrombocytopenia adequately determines the improved hemostatic activity by transfused platelets.
\end{abstract}




\section{Introduction}

Platelet transfusion is a mainstream therapy for preventing or treating bleeding episodes in patients with low platelet counts, e.g. after high-dose chemotherapy. Although there is controversy as to whether prophylactic platelet transfusion is necessary, these patients are currently subjected to transfusion as soon as the platelet concentration falls below $10-20 \times 10^{9}$ platelets/L. The efficacy of platelet transfusions is usually evaluated from the corrected count increment $(\mathrm{CCl})$ of platelets at $1 \mathrm{~h}$ after transfusion. ${ }^{241}$ This test reports on the presence of transfused platelets in the circulation, but unfortunately does not evaluate their hemostatic activity. Accordingly, measurement of the $\mathrm{CCl}$ alone appears to be insufficient to determine the risk of haemorrhage. ${ }^{73}$ Several factors are known to affect the activity of circulating platelets including clinical variables, such as fever, medication and the presence of antibodies, and intervention variables, such as the storage medium and the quality of the transfusion product. ${ }^{67,78,116}$ It is thus desirable to search for functional tests that can assess hemostatic activity before and after transfusion, with the aim to better minimise the risk of bleeding.

Currently, only a few tests are available to measure the responsiveness of platelets under thrombocytopenic conditions. Assessment of the bleeding time as a measure of transfusion outcome has a poor predictive value. ${ }^{242}$ Conventional functional measurements, such as turbidometric aggregation and shear-induced occlusion (using a platelet function analyzer, PFA-100; Dade Behring, Newark, DE, USA), cannot be applied, as these rely on the presence of high platelet concentrations. ${ }^{243,244}$ Flow cytometry is useful to detect activation markers at the platelet surface even at low platelet counts. ${ }^{245,246}$ However, this method does not report on platelet-platelet interactions and, hence, is not a measure of overall hemostasis. Rotational thromboelastography has recently been developed to assess fibrin clot formation in whole blood, but this method has low sensitivity to platelet function. Due to these limitations, little is currently known about the functional activity of circulating platelets after transfusion in thrombocytopenic patients. There is thus a need for new tests to 
study platelet function in thrombocytopenia, preferably operating at physiologically relevant conditions.

We have previously described an in vitro assay to measure platelet adhesion and thrombus formation in whole blood under flow conditions, which reports on the collagen-, ADP- and thromboxane-induced responses of platelets under physiologically relevant conditions..$^{88,90,247}$ Another test was developed to study the coagulation-promoting (procoagulant) effect of activated platelets in plasma via continuous measurement of thrombin generation. ${ }^{102,248}$ The present study aimed to adapt these assays to determine the hemostatic activity of blood at low platelet counts such as in thrombocytopenia. Subsequently, these tests were evaluated for identifying patients with aberrant hemostatic activity following platelet transfusion.

\section{Materials and methods}

\section{Patients}

Thirty-eight patients with acute myeloid leukemia, (non-)Hodgkin lymphoma or multiple myeloma were studied, who were consecutively admitted to the hospital. The patients had a median age of 53 years (range 22-77 years); 21 patients were male and 17 female. Further inclusion criteria were absence of human leucocyte antigen (HLA) or non-HLA antibodies, no use of anti-platelet or anti-coagulant medication, and hematocrit levels $\geq 20 \%$. All patients were on high-dose chemotherapy with ciprofloxacine and fluconazol, and received platelet transfusion support for prophylactic reasons during bone marrow aplasia. One of the patients developed acute graft-versus-host disease. This patient was treated with ciclosporin and prednisone, and later received four platelet transfusions. Another patient was treated with amphotericin B as additional medication.

None of the patients experienced a bleed during at least $48 \mathrm{~h}$ following transfusion, as examined by the medical staff. Before transfusion, the mean platelet concentration in blood was $9.4 \pm 5.7 \times 10^{9}$ platelets/L (range 4$28 \times 10^{9} / \mathrm{L}$ ), while the hematocrit was $23.7 \pm 2.3 \%$. Of the 38 patients, 17 received transfusion with $300 \mathrm{~mL}$ platelets in plasma (unit of $300 \times 10^{9}$ 
platelets), while 21 patients were transfused with $300 \mathrm{~mL}$ platelets in platelet additive solution II (PAS-II) medium (unit of $300 \times 10^{9}$ platelets). The transfusions were with $\mathrm{ABO}$-matched platelet preparations, except for one case, where an $A B$ patient received a blood group $A$ preparation. Transfusions with $300 \mathrm{~mL}$ of platelet concentrates were completed for $90-95 \%$.

Clinical efficacy of the transfusion was expressed as the corrected platelet $\mathrm{CCl}$. The $\mathrm{CCl}$ is defined as: (platelet count increment $\times$ body surface area)/(number of transfused platelets $\times 10^{11}$ ). After $1 \mathrm{~h}$, the mean $\mathrm{CCl}$ was $15.2 \pm 7.4(n=38)$, pointing to overall successful treatment according to the National Blood Transfusion guidelines (Quality Institute for Health Care in the Netherlands, CBO). ${ }^{68}$ Studies were approved by the Medical Ethical Committee of the University and Hospital of Maastricht; patients and healthy subjects gave full informed consent.

\section{Preparation of platelet concentrates for transfusion}

Platelets for transfusion were prepared according to two procedures, and stored at standard blood bank conditions. ${ }^{100}$ In one procedure, citratephosphate-dextrose (CPD) anti-coagulated donor blood was centrifuged, after which plasma and erythrocytes were removed. Platelet-rich buffy coats from five donors were then pooled with the plasma from one donor; leucocytes were removed by filtration. The pooled platelet-plasma preparations (about $300 \mathrm{~mL}$ ), containing about $300 \times 10^{9}$ platelets $\left(>250 \times 10^{9}\right.$ according to standard blood bank procedures) and $<1 \times 10^{6}$ leucocytes, were stored at $22^{\circ} \mathrm{C}$ in a flatbed shaker according to National Blood Transfusion guidelines. In the other procedure, about $300 \times 10^{9}$ leucocyte-free platelet pools from five donors were stored in $30 \%$ plasma and $70 \%$ synthetic medium $\left(>250 \times 10^{9}\right.$ platelets and $<1$ $\times 10^{6}$ leucocytes per $300 \mathrm{~mL}$ ). The synthetic medium used was PAS-II, containing $116 \mathrm{mM}$ sodium chloride, $10 \mathrm{mM}$ trisodium citrate and $30 \mathrm{mM}$ sodium acetate (Baxter, Utrecht, the Netherlands). Storage of the platelets in plasma and in synthetic medium was for $4.5 \pm 2.3$ and $4.3 \pm 1.2 \mathrm{~d}$, respectively. 


\section{Collection of blood}

A vessel in the forearm of healthy volunteers was punctured with a $1.2 \mathrm{~mm}$ needle (Braun, Melsungen, Germany), and freely flowing blood was collected into $10 \% 0.129 \mathrm{M}$ trisodium citrate. Blood from thrombocytopenic patients was obtained via a central venous catheter. The first $5-10 \mathrm{~mL}$ of patient blood was discarded, after which flowing blood was collected into $10 \%$ of $0.129 \mathrm{M}$ trisodium citrate. Samples of patient blood were obtained immediately before and at $1 \mathrm{~h}$ after transfusion. Platelet and erythrocyte (hematocrit) concentrations were determined with a cell counter (Coulter Electronics, Luton, UK).

\section{Preparation of platelets and plasma}

Platelet-rich plasma (PRP) was prepared by centrifugation of citrate anticoagulated whole blood at $240 \mathrm{~g}$ for $15 \mathrm{~min}$ at room temperature. Platelet-poor plasma (PPP) was obtained by centrifuging PRP twice at $2650 \mathrm{~g}$ for $10 \mathrm{~min}$. For obtaining platelet-free plasma (PFP), PRP was centrifuged at $20,000 \mathrm{~g}$ for 5 min. When plasma with low platelet counts was needed, the PRP was diluted with autologous PPP as appropriate.

\section{Preparation of reconstituted thrombocytopenic blood}

Citrated blood from healthy subjects was centrifuged at $2650 \mathrm{~g}$ for $10 \mathrm{~min}$. The lower phase, enriched in erythrocytes, was combined with different volumes of autologous PRP and PPP, to produce reconstituted blood samples with defined numbers of erythrocytes and platelets. Platelet and erythrocyte counts were determined in the reconstituted samples. Where indicated, (pretransfusion) blood from a thrombocytopenic patient was supplemented in vitro with the remaining platelets from the same concentrate as was given to that patient. Platelets were reconstituted to correspond to the platelet concentration of the post-transfusion blood of the same patient.

\section{Whole blood flow experiments}

Before starting flow experiments, citrate anti-coagulated blood from healthy donors or patients was recalcified with $16.6 \mathrm{mM} \mathrm{CaCl}_{2}$ in the presence of $20 \mu \mathrm{M}$ 
D-Phe-Pro-Arg chloromethyl ketone (final concentration, f.c., PPACK; Calbiochem, San Diego, CA, USA) to avoid coagulation. Coverslips were coated with Horm-type fibrillar collagen (Nycomed, Munich, Germany) and mounted onto a transparent, parallel-plate flow chamber that was placed on the stage of a fluorescence microscope. The chamber (inner dimensions: $50 \mu \mathrm{m}$ deep and $3 \mathrm{~mm}$ wide) was designed for use of small blood volumes $(<0.4 \mathrm{~mL}$ per experiment). The blood was perfused at a continuous wall-shear rate of 1000/s, as described ${ }^{90,91}$ After perfusion, high-resolution phase-contrast images were recorded in real-time with a Visitech digital imaging system (Sunderland, UK). Images from $>15$ adjacent collagen-containing microscopic fields were analysed for surface area coverage with adherent and aggregated platelets, using ImagePro software (Media Cybernetics; Silver Spring, MD, USA). To analyse phosphatidylserine (PS) exposure, platelets on coverslips were stained post-perfusion with $0.25 \mu \mathrm{g} / \mathrm{mL}$ annexin A5-fluorescein isothiocyante (FITC) (Nexins, Hoeven, the Netherlands). ${ }^{88}$ Using an automated shutter control, fluorescence images were taken from representative microscopic fields, and analysed with Quanticell software (Visitech). Where indicated, the blood was pre-incubated with the $\mathrm{P}_{2} \mathrm{Y}_{1}$ receptor blocker, MRS-2179 at $100 \mu \mathrm{M}$ (Sigma, St Louis, MO, USA) and with the $\mathrm{P}_{2} \mathrm{Y}_{12}$ receptor blocker AR-C69931MX at $20 \mu \mathrm{M}$ (AstraZeneca, Mölndal, Sweden).

\section{Thrombin generation measurements}

In citrated PRP from healthy donors or thrombocytopenic patients, thrombin generation was assayed using an automated calibrated thrombogram method. ${ }^{102,249}$ Briefly, triplicate samples of $80 \mu \mathrm{L}$ PRP were pipetted into the wells of a 96-well plate (Immulon 2HB; Dynex Technologies, Chantilly, VA, USA), containing $20 \mu \mathrm{L}$ tissue factor (6 pM, Dade Behring, Mannheim, Germany) in buffer A [20 mM Hepes, $140 \mathrm{mM} \mathrm{NaCl}$ and $5 \mathrm{mg} / \mathrm{mL}$ bovine serum albumin (BSA), pH 7.35]. Plates were inserted into a Fluoroskan Ascent wellplate reader (Thermolab Systems, Helsinki, Finland), and pre-heated to $37^{\circ} \mathrm{C}$ for 5 min. Coagulation was started by automated addition of $20 \mu \mathrm{L}$ of $2.5 \mathrm{mM}$ ZGly-Gly-Arg aminomethyl coumarin (Z-GGR-AMC, Bachem, Bubendorf, 
Switzerland), dissolved in buffer B (20 mM Hepes, $0.1 \mathrm{M} \mathrm{CaCl}_{2}$ and $60 \mathrm{mg} / \mathrm{mL}$ $\mathrm{BSA}, \mathrm{pH}$ 7.35). Plates were shaken for $10 \mathrm{~s}$ after substrate addition. Where indicated, the PRP was pre-activated with $20 \mu \mathrm{M}$ ADP (Sigma) or $5 \mu \mathrm{g} / \mathrm{mL}$ collagen (Nycomed). Thrombin generation in PFP was measured in the presence of $4 \mu \mathrm{M}$ phospholipid vesicles (PS: phosphatidyl choline: phosphatidyl ethanolamine, 1:3:1, molar ratio) after triggering with $10 \mathrm{pM}$ tissue factor. The increase in fluorescence from cleaved AMC was measured continuously at an excitation wavelength of $390 \mathrm{~nm}$ and an emission wavelength of $460 \mathrm{~nm}$. Firstderivative curves were calculated from the accumulation in fluorescence, corrected for $\alpha_{2}$-macroglobulin-bound thrombin, and converted into $\mathrm{nM}$ thrombin concentrations using a human thrombin calibrator (Thrombinoscope, Maastricht, the Netherlands). Thrombogram software (Synapse, Maastricht, the Netherlands) was used to obtain calibrated thrombin concentration curves. Platelet-dependent thrombin generation was evaluated from the increase in thrombin peak height. This thrombogram parameter is an indicator of the maximal rate of thrombin formed and is particularly sensitive to the platelet count.

\section{Flow cytometry}

Citrated whole blood from thrombocytopenic patients was diluted ten times with phosphate-buffered saline, and platelets were activated with ADP and SFLLRN to measure P-selectin expression (FITC-anti-CD62) or integrin $\alpha$ llb $\beta 3$ activation (FITC-PAC1), as described. ${ }^{100}$ Scatter regions corresponding to platelets were set to determine binding of fluorescent antibodies. Data were analysed with WinMDI 2.8 software.

\section{Statistical methods}

Statistical comparison of (un)paired data was performed with the Statistical Package for Social Sciences, version for Windows (SPSS, Chicago, IL, USA), using paired or unpaired $t$-tests. Analysis of correlations between two populations was performed with the Pearson Correlation test. Data are mean \pm standard deviation (SD), unless indicated otherwise. 


\section{Results}

\section{Effect of platelet transfusion to thrombocytopenic patients}

Thirty-eight patients with severe thrombocytopenia were transfused prophylactically with one unit of about $300 \times 10^{9}$ platelets in either plasma ( $n=17$ ) or synthetic PAS-II medium $(n=21)$. Efficacy of the intervention was determined from the increase in platelet count and the $\mathrm{CCl}$, using blood samples taken before and at $1 \mathrm{~h}$ after transfusion. After the intervention, patients who received platelets in plasma showed an increase in platelet concentration from $8.7 \pm 5.7$ to $35.3 \pm 14.8 \times 10^{9}$ platelets/L (range from 4-18 to $\left.7-70 \times 10^{9} / \mathrm{L}\right)$. The hematocrit post-transfusion was $22.9 \pm 2.4 \%$. For patients who were transfused with platelets in synthetic medium, the platelet concentration increased from $10.1 \pm 5.8$ to $34.6 \pm 14.9 \times 10^{9}$ platelets/L (range from $4-28$ to $10-76 \times 10^{9} / \mathrm{L}$ ). The hematocrit post-transfusion was $24.9 \pm 1.6 \%$. After $1 \mathrm{~h}$, the $\mathrm{CCl}$ of these two patient groups amounted to $17.6 \pm 2.4$ and $14.4 \pm 8.1 \quad(P=0.6)$, respectively, thus indicating the average successful transfusion.

\section{Flow-induced platelet adhesion and thrombus formation in blood with low platelet concentration}

Flow experiments with blood from healthy subjects were performed to determine how the thrombus-forming process depends on the platelet concentration. Therefore, reconstituted whole blood, containing different concentrations of platelets $\left(5-300 \times 10^{9}\right.$ platelets/L) and a fixed number of erythrocytes ( $40 \%$ hematocrit), was perfused through a transparent, capillarysize flow chamber. Perfusion over collagen was at a shear rate of $1000 / \mathrm{s}$ for $240 \mathrm{~s}$, i.e. a time period where platelet deposition on collagen increases linearly with time. ${ }^{88}$ To approach physiological, millimolar concentrations of free $\mathrm{Mg}^{2+}$ and $\mathrm{Ca}^{2+}$, the citrated blood was recalcified immediately before perfusion, while PPACK was added to completely prevent coagulation. Phase-contrast images at the end of the perfusion period showed that the number of adherent platelets increased with the concentration of platelets in the reconstituted blood. Aggregates of platelets started to form at $>30 \times 10^{9}$ platelets/L (figure $1 \mathrm{~A}$ ). 
Measurement of the surface area covered by adherent platelets indicated a progressive increase with the platelet concentration (figure 1B).

Thrombocytopenic patients treated for leukemia often have reduced hematocrit values. To mimic this condition, the flow experiments were repeated using reconstituted blood containing 20 vol\% erythrocytes and platelets in the range of $5-60 \times 10^{9} / \mathrm{L}$ (figure $1 \mathrm{C}$ ). Off-line analysis of contrast images showed that the surface area covered by adherent platelets was linearly related to the platelet count $\left(R^{2}=0.98, P=0.001\right)$. Donor-to-donor variation was relatively small (CV of $10 \%, n=3$ ). Increasing the hematocrit value to $40 \%$ resulted in a moderately higher platelet deposition (figure 1C, open symbol). This confirmed that red cells stimulate platelet-surface contact under flow. ${ }^{250,251}$ Furthermore, this experiment also indicated that the concentration of platelets was the major variable determining platelet deposition to collagen in this flow-chamber test.

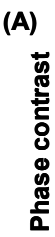

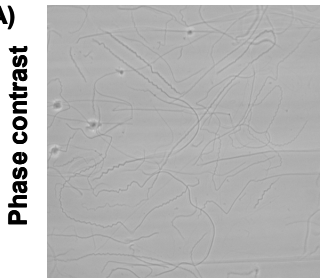

$5 \times 10^{9}$

(B)

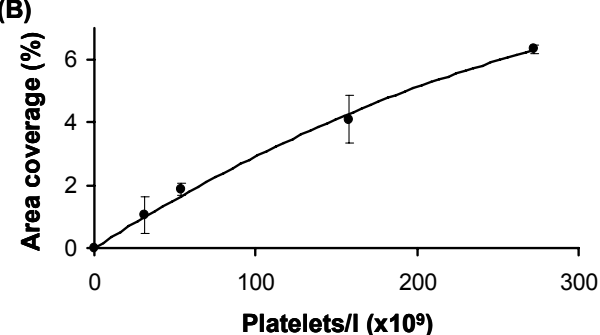

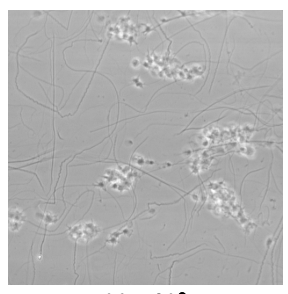

$60 \times 10^{9}$

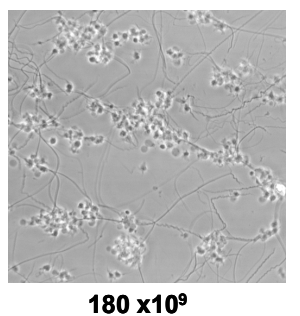

(C)

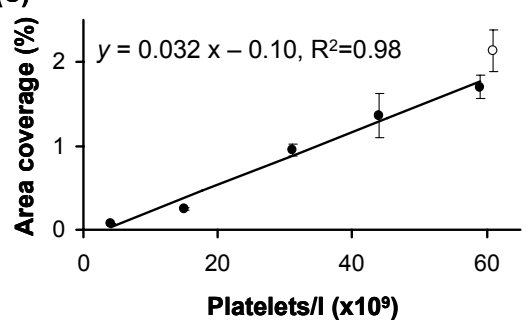

Figure 1. Platelet concentration-dependent adhesion to collagen under flow. Reconstituted blood from healthy volunteers was perfused over a collagen surface at a shear rate of 1000/s for 240 s. Citrated blood was used, which was recalcified in the presence of D-Phe-Pro-Arg chloromethyl ketone (PPACK) anti-coagulant. (A) Representative images of platelet deposition after perfusion with blood containing $5-180 \times 10^{9}$ platelets/L (hematocrit $40 \%$ ). Images represent $120 \times 120 \mu \mathrm{m}$. (B) Surface area coverage with platelets after perfusion $(n=5)$. (C) Linearity of surface area coverage up to $60 \times 10^{9}$ platelets/L. Closed and open symbols represent data at hematocrit values of $20 \%$ and $40 \%$ respectively ( $n=3$ independent measurements). 


\section{Effect of platelet transfusion on platelet adhesion and thrombus formation under flow}

Platelet adhesion and thrombus formation on collagen was then measured in flow studies using pre- and post-transfusion blood from several thrombocytopenic patients (transfused with platelets in plasma). Recalcified blood samples, containing PPACK, were perfused over immobilised collagen for $240 \mathrm{~s}$. In case of pre-transfusion blood, only few platelets adhered to the collagen surface, but with post-transfusion blood many more platelets adhered and formed small thrombi (figure 2A). The platelet aggregation was due to the release of autocrine ADP, as pre-incubation of the post-transfusion blood with ADP receptor antagonists (MRS-2179 to block P2Y 1 and AR-C69931MX to block $\mathrm{P} 2 \mathrm{Y}_{12}$ receptors) abolished the formation of thrombi without influence on the adhesion. Thus, surface area coverage with platelets was only partly reduced under this blocking condition (figure $2 \mathrm{~B}$ ).

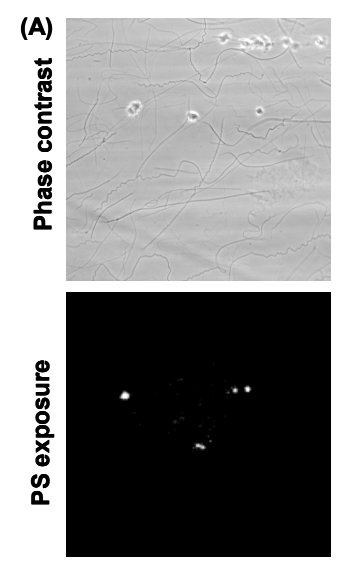

Before transfusion

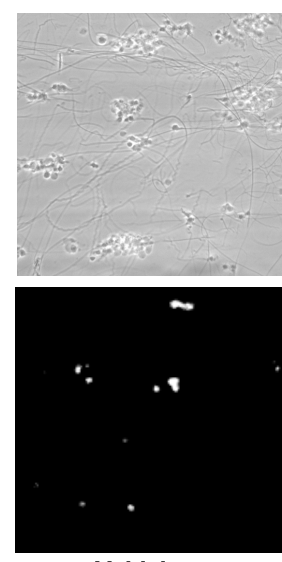

Vehicle
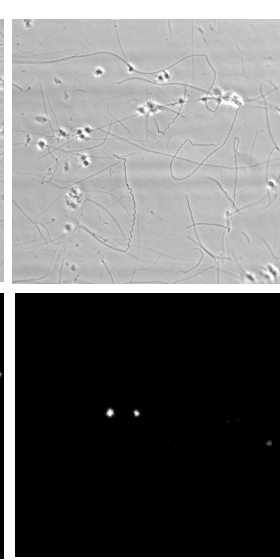

ADP-R block
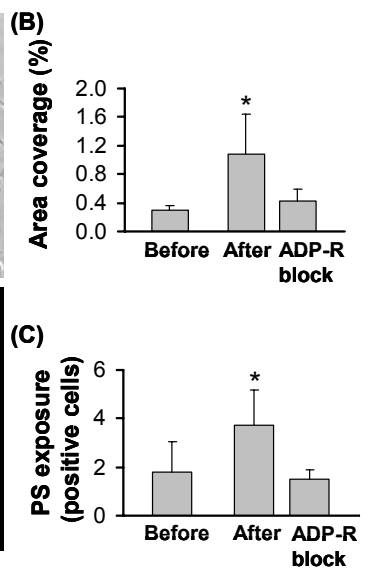

Figure 2. Flow-induced activation of platelets after transfusion. Recalcified blood from thrombocytopenic patients was perfused over collagen, as described for figure 1. (A) Shown are representative images of platelet deposition (upper panels, $120 \times 120 \mu \mathrm{m}$ ) and of staining with annexin A5-fluorescein isothiocyante (FITC) to mark activated, phosphatidylserine (PS)-exposing platelets (lower panels, $150 \times 150 \mu \mathrm{m}$ ). Platelet counts before and after transfusion with platelets in plasma were 8 and $70 \times 10^{9} / \mathrm{L}$ respectively. Quantitative effects of ADP receptor blockage on platelet adhesion (B) and PS exposure (C) under flow. Post-transfusion blood was pre-incubated with ADP receptor blockers MRS-2179 and AR-C69931MX, as indicated. Mean platelet counts were 10.3 and $55.0 \times 10^{9} / \mathrm{L}$ pre- and post-transfusion respectively ( $n=3$ patients). ${ }^{*} P<0.1$ compared with pre-transfusion. 
The procoagulant activity of the collagen-adhered platelets was determined, as a typical glycoprotein (GP)VI-dependent response, ${ }^{90,91}$ by post-staining with the PS-binding probe, annexin A5-FITC. In case of post-transfusion blood, a substantial number of platelets adhered to collagen-bound annexin A5 (figure $2 A$ ). Again, blocking of the ADP receptors lowered this platelet response (figure $2 \mathrm{C})$. Together, this indicated that the collagen and ADP receptors of the transfused platelets had kept their responsiveness after the transfusion. Furthermore, these results emphasized the importance of these receptors in the thrombus-forming process, even at low platelet concentrations.

In a larger size study, perfusion experiments over collagen were performed with pre- and post-transfusion blood samples from 38 thrombocytopenic patients. On average, in this flow test, higher platelet concentrations after transfusion (figure $3 \mathrm{~A}$ ) were accompanied by increased platelet deposition (figure 3B). A similar increase in platelet adhesion was obtained in reconstitution experiments, when the platelets from transfusion bags were added in vitro to pre-transfusion blood (to the same count as present in posttransfusion samples). For all patients together $(n=38)$, the surface area coverage increased from $0.19 \pm 0.13 \%$ to $0.66 \pm 0.41 \%$ after transfusion and to $0.54 \pm 0.28 \%$ after reconstitution. Although in vitro reconstitution gave a similar increase in surface area coverage as achieved by transfusion (figure 3B), in a marked number of cases the adhesion pattern of platelets to the surface was different. In $40 \%$ of the analysed images, numbers of adhered platelets were similar, but the platelet aggregates of post-transfusion samples were larger in size than in reconstituted samples; this points to a recovery of the integrin activation mechanism in the transfused platelets.

There were no marked differences between blood samples from patients, who were transfused with platelets stored in plasma $(n=17)$ or with platelets stored in synthetic medium $(n=21)$. The mean surface area coverage after transfusion with either type of platelet preparation was $0.63 \pm 0.30 \%$ and $0.68 \pm 0.48 \%$ respectively. Plotting the transfusion effects on platelet count and surface area coverage for all patients gave a strong linear relation, i.e. $y=0.012 x+0.11\left(R^{2}=0.40, P=0.001\right.$, figure $\left.3 C\right)$. A similar linear relationship 

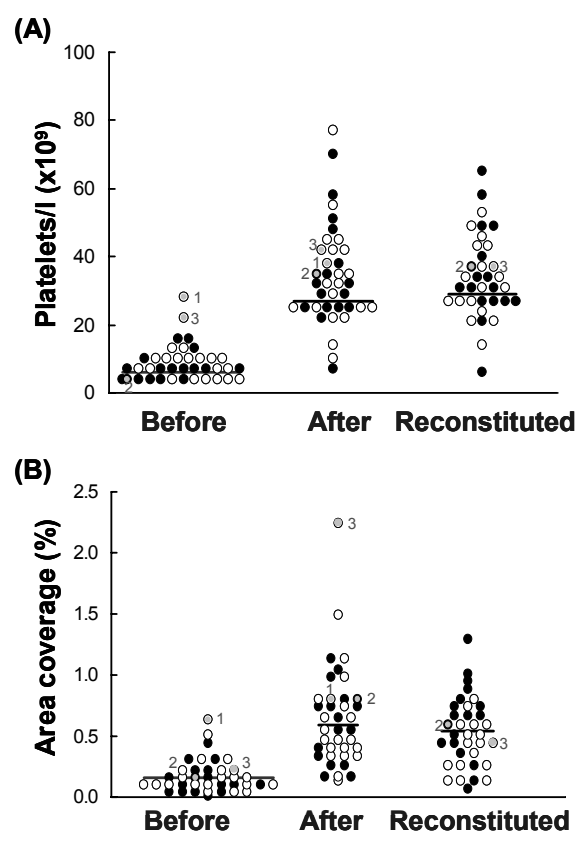

(C)

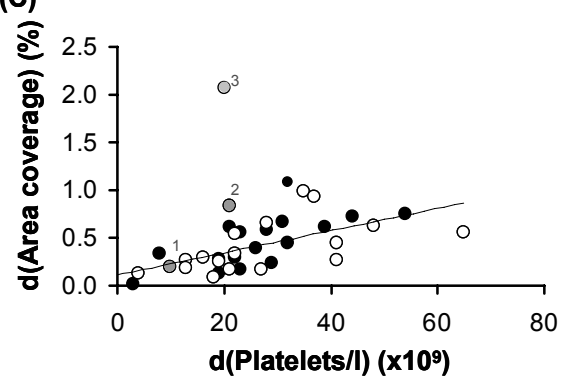

Figure 3. Effect of platelet transfusion on platelet adhesion to collagen under flow. Recalcified whole blood from thrombocytopenic patients was obtained before and at $1 \mathrm{~h}$ after transfusion with platelets stored in plasma (closed symbols, $n=17$ ) or platelets stored in synthetic medium (open symbols, $n=21$ ). Pre-transfusion blood was also reconstituted in vitro with platelets from the same preparation to reach the posttransfusion count. Perfusion experiments over collagen were performed as described in figure 1. (A) Platelet concentration in blood before and after transfusion. (B) Surface area coverage of coverslips with platelets after perfusion of pre- and posttransfused blood. (C) Correlation between increase in platelet concentration and increase in surface area coverage due to transfusion. Transfusion with platelets stored in plasma: $\quad R^{2}=0.57 \quad(P=0.001)$; transfusion with platelets stored in synthetic medium: $\quad R^{2}=0.33$ $(P=0.008)$. Patient 1 received additional medication (amphotericin B), patient 2 experienced graft-versus-host disease, patient 3 had exceptionally high thrombus formation after transfusion.

was obtained when comparing patients who were transfused with platelets in plasma $\left(R^{2}=0.57, P=0.001\right)$ with those transfused with platelets in synthetic medium $\left(R^{2}=0.33, P=0.008\right)$.

\section{Thrombin generation in plasma at low platelet concentrations}

The coagulation-promoting effect of platelets can be well estimated from thrombin generation curves using PRP that is triggered with tissue factor and $\mathrm{CaCl}_{2}$. We investigated the experimental conditions at which thrombin generation varied most strongly with the platelet concentration in PRP from healthy subjects. In the presence of a low tissue factor concentration (1 pM), 
peak levels of thrombin increased consistently with the platelet count up to $240 \times 10^{9} / \mathrm{L}$ (figure $4 \mathrm{~A}$ ). As this assay is known to detect subject-dependent differences in activity of the coagulation system per se, ${ }^{102}$ it was necessary to correct for this platelet-independent variation. Therefore, controls were performed with each plasma sample (PFP, no platelets), where the thrombin generation process was maximal by adding procoagulant phospholipid vesicles $(4 \mu \mathrm{M})$ and triggering with a high dose of tissue factor (10 pM) plus $\mathrm{CaCl}_{2}$. The maximal thrombin peak level obtained was then used to normalise the peaks of curves with platelets (figure 4A). Plotting of normalised thrombin peak levels as a function of the platelet concentration gave a linear relationship in the range of $0-60 \times 10^{9}$ platelets $/ \mathrm{L}\left(R^{2}=0.98, P=0.008\right)$ (figure $\left.4 \mathrm{~B}\right)$. These normalised thrombin generation curves were used to assess the procoagulant activity of platelets in plasma from thrombocytopenic patients.

\section{Effect of platelet transfusion on thrombin generation in PRP}

Platelet-containing plasma (control PRP) from patients obtained before and

(A)

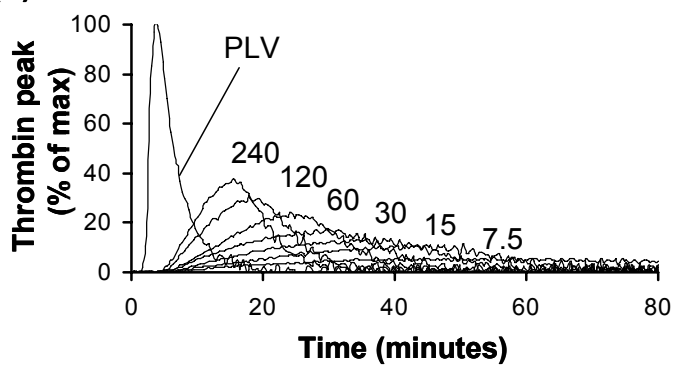

(B)

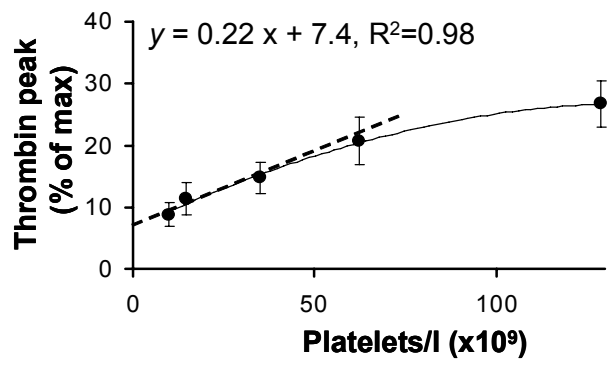

Figure 4. Platelet concentrationdependent thrombin generation in platelet-rich plasma. (A) Control citrate anti-coagulated plasma with indicated platelet concentrations was triggered with recombinant tissue factor (1 pM) and $\mathrm{CaCl}_{2}(16.6 \mathrm{mM})$, and thrombin generation was measured with fluorescent substrate. Maximal thrombin peak levels $(100 \%)$ were obtained by triggering a platelet-free plasma sample with high tissue factor $(10 \mathrm{pM})$ in the presence of phospholipid vesicles $(4 \mu \mathrm{M}$, PLV curve). Traces are representative of $>5$ experiments. (B) Effect of platelet concentration in plasma on thrombin peak level. Data are expressed as percentages of the maximal peak level (PLV curve). Peak levels were linearly correlated with platelet count in the range of $5-60 \times 10^{9}$ platelets/L $\left(R^{2}=0.98, \quad P=0.008\right)$. Note that traces of thrombin are formed in the absence of platelets. 
after transfusion gave thrombin generation curves that, in many but not all individuals, showed earlier and higher thrombin peak levels after transfusion. This is illustrated in figure $5 A$ and $B$, showing increased thrombin generation for a patient, whose platelet count increased from 8 to $33 \times 10^{9}$ platelets/L after transfusion. In the post-transfusion PRP, the reactivity of platelets was monitored by stimulation with ADP or collagen, before triggering of the coagulation with tissue factor/ $\mathrm{CaCl}_{2}$. In patient plasmas, ADP had a minimal stimulating effect on the thrombin peak in comparison with collagen (figure 5B). However, in PRP samples from control subjects, diluted with plasma to a low platelet count $\left(30 \times 10^{9}\right.$ platelets/L), ADP had an accelerating effect on thrombin generation, though this was less than seen with collagen (figure $5 \mathrm{C}$ ).

(A)

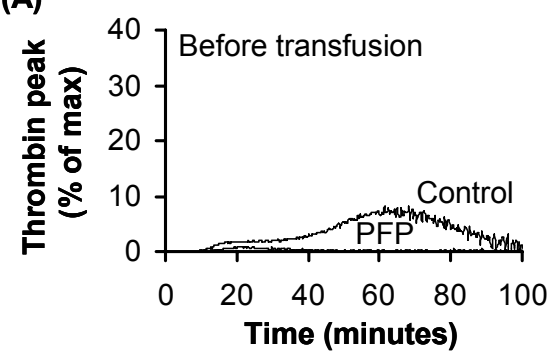

(B)

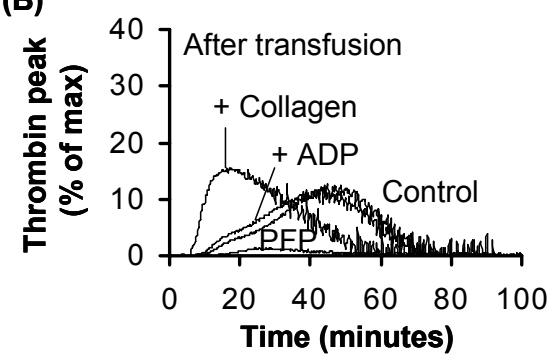

(C)

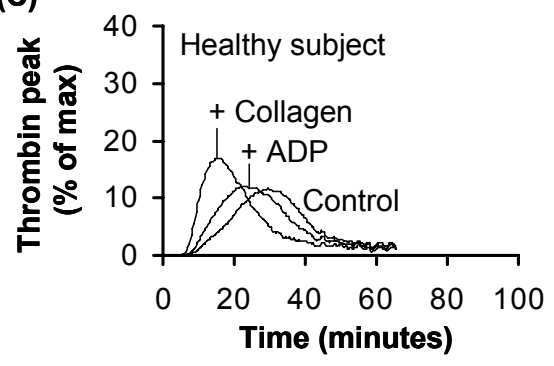

Figure 5. Increased platelet activation-dependent thrombin generation after transfusion. Platelet-rich plasma (PRP) was prepared from thrombocytopenic patients before $(A)$ and after (B) transfusion with platelets in plasma. Representative curves of thrombin generation are shown of a patient with platelet counts in PRP of 8 and $33 \times 10^{9} / \mathrm{L}$ before and after transfusion respectively. The PRP was unstimulated (control) or preactivated with ADP $(20 \mu \mathrm{M})$ or collagen $(5 \mu \mathrm{g} / \mathrm{mL})$, prior to addition of tissue factor and $\mathrm{CaCl}_{2}$. Autologous platelet-free plasma (PFP) was used as a control. Thrombin activities are given as percentages of the maximal thrombin peak with phospholipid vesicles, as described for figure 4. (C) Representative thrombin generation curves of reconstituted PRP $\left(30 \times 10^{9}\right.$ platelets/L) from a healthy subject; platelet activation was as for panel B. 
Thrombin generation curves were measured in (un)stimulated PRP samples obtained from 11 patients receiving transfusion with platelets stored in plasma and from 18 patients transfused with platelets stored in synthetic medium. Plasmas from remaining patients could not be used due to the presence of traces of heparin. In both groups of patients, increases in platelet concentration after transfusion (figure $6 \mathrm{~A}$ ) were generally accompanied by higher thrombin peak levels (figure 6B). The transfusion effects on platelet concentration and (normalised) thrombin peak level were closely correlated in cases of transfusion with platelets stored in plasma $(P=0.033)$ or in synthetic medium $(P=0.002$, figure $6 \mathrm{C})$. Enhancing effects of ADP and collagen on thrombin generation were variable but appeared to be correlated, when evaluated for the patients transfused with platelets stored in plasma $(P=0.004$, figure $6 D)$. However, this was not the case after transfusion with platelets stored in synthetic medium $(P=0.097)$. Accordingly, on average, an increase in platelet count after transfusion was accompanied by a corresponding increase in plateletdependent coagulation, in spite of the fact that individual samples deviated from this main trend. Further, it appears that transfusion of platelets in synthetic medium leads to a diminished responsiveness of the cells.

\section{Aberrant thrombus formation or platelet-dependent coagulation in specific thrombocytopenic patients}

One of the patients was prophylactically treated with amphotericin $B$, which is known to reduce the efficacy of platelet transfusion. In this patient, the platelet count increased slightly, from 28 to $38 \times 10^{9}$ platelets/L after transfusion, which was accompanied by a small increase in thrombus formation (figure 3, patient 1 ), but not in thrombin generation (figure 6, patient 1 ).

We separately examined blood samples from a thrombocytopenic patient with acute graft-versus-host disease, who was transfused four times with platelet preparations at 1-week intervals. This enabled the evaluation of the effect of repetitive transfusions in the same patient. On average, at $1 \mathrm{~h}$ after each transfusion, thrombus formation was increased in a manner corresponding with the mean higher platelet count (figure 7). However, thrombin generation in all 

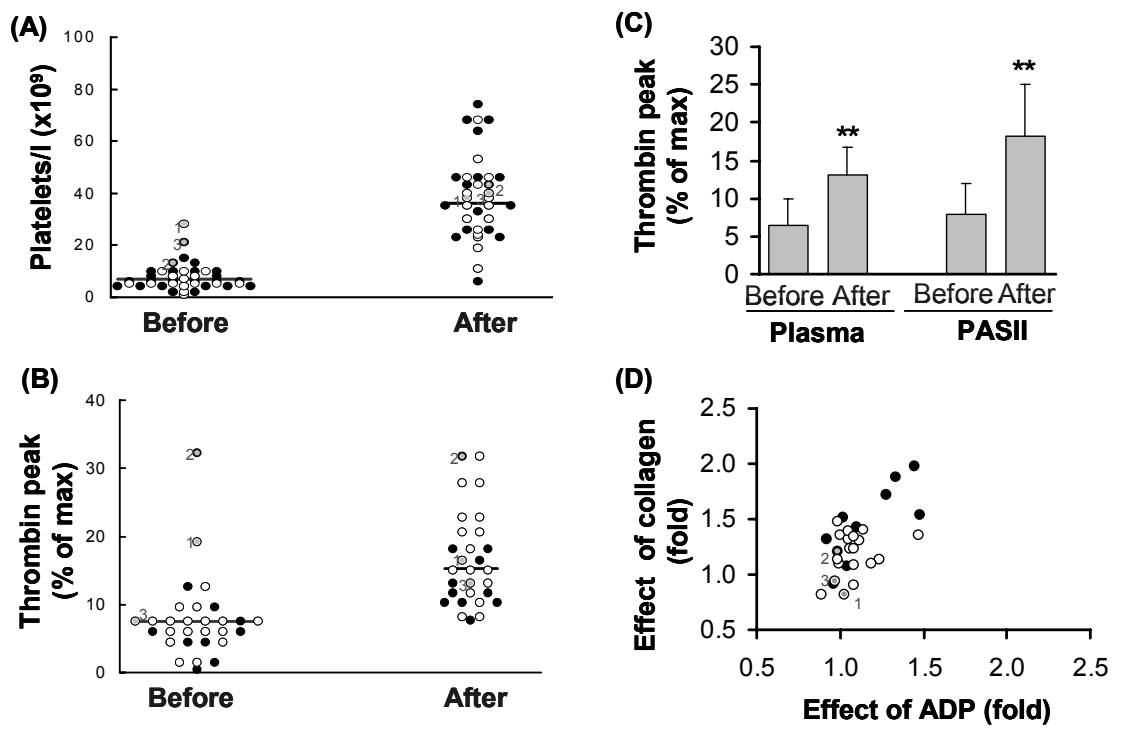

Figure 6. Effect of platelet transfusion on thrombin generation. Thrombin generation was measured in platelet-rich plasma (PRP) from thrombocytopenic patients before and after transfusion with platelets in plasma (closed symbols, $n=11$ ) or platelets in synthetic medium (open symbols, $n=18$ ). (A, B) Platelet concentration and thrombin peak levels (\% of maximal) in PRP before and after transfusion with platelets stored in plasma (closed symbols) or in synthetic medium (PAS-II, open symbols). (C) Effect of transfusion on thrombin peak levels in PRP. (D) Effects of ADP and collagen on thrombin generation in PRP after transfusion with platelets stored in plasma (closed) or in synthetic medium (open). Data are fold increase in comparison to thrombin peak levels in the absence of ADP/collagen. Patient 1 received additional medication (amphotericin B), patient 2 experienced graft-versus-host disease, patient 3 had exceptionally high thrombus formation after transfusion. ${ }^{* *} p<0.05$ compared with pre-transfusion.

four PRP and PFP samples was already high before transfusion, and did not further increase afterwards. Coagulation parameters in plasma were in the normal range for this patient [aPTT of $23 \mathrm{~s}$, prothrombin time of $10.5 \mathrm{~s}$, and International Normalized Ratio (INR) of 1.0], thus indicating normal activity of the coagulation system.

Intriguingly, post-transfusion blood samples from another patient gave a relatively high thrombus formation after perfusion (figure 3 , patient 3 ), which was confirmed in triplicate measurements. Thrombin generation measurements - using the same blood samples - were all in the normal range. There was no obvious reason for the increased thrombus-forming activity in the blood from this patient. However, this result shows that the present flow test could detect abnormalities in platelet function after transfusion. 
(A)

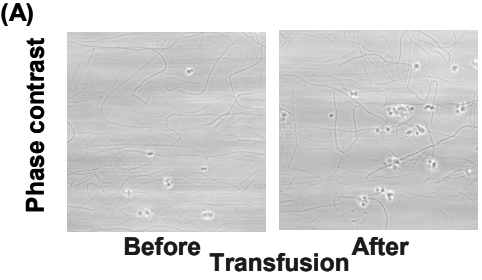

(C)

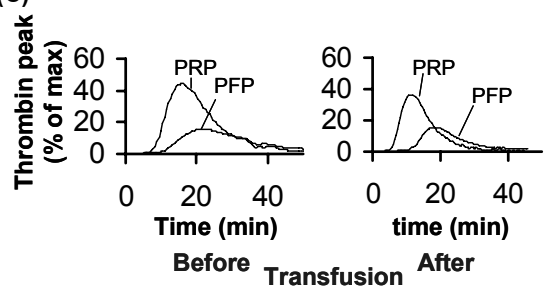

(B)

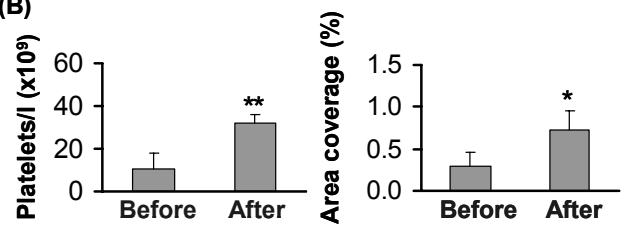

(D)

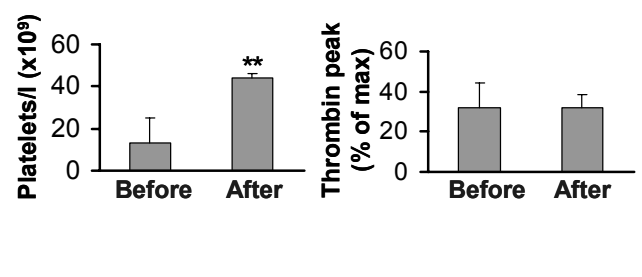

Figure 7. Effect of platelet transfusion on thrombus formation and thrombin generation in a patient with graft-versus-host disease. Blood from a thrombocytopenic patient with graft-versushost disease was obtained before and after multiple transfusions with platelets. (A) Representative images of flow-induced platelet deposition on collagen before and after perfusion. (B) Transfusion effect on platelet concentration in blood and thrombus formation under flow $(n=4)$. (C) Representative thrombin generation curves with platelet-rich plasma (PRP) and platelet-free plasma (PFP) of this patient before and after transfusion. (D) Transfusion effect on platelet concentration in PRP and normalised thrombin peak levels $(n=3)$. ${ }^{*} p<0.1,{ }^{* *} p<0.05$ compared with pre-transfusion.

\section{Discussion}

Platelet transfusion is a common strategy in the supportive care of patients who develop thrombocytopenia as a result of chemotherapy. A significant number of platelets administered in clinical settings are used for prophylactic transfusion to this group of patients. The success of such a transfusion is currently mainly or solely assessed from the increase in the $\mathrm{CCl}$. Although primarily a transfusion parameter, the $\mathrm{CCl}$ has been used to predict cellular viability of the transfused platelets. ${ }^{252}$ The present study investigated the suitability of two tests that may directly assess the improved hemostatic capacity after the transfusion of platelets stored in various media. As a measure of platelet function under conditions of flow, we monitored collagen-induced thrombus formation under high shear stress. Secondly, we measured the potency of platelets to support thrombin generation in coagulating plasma. The results of the first assay indicate that transfused platelets, after circulation in the patient blood, could still firmly adhere to collagen and form aggregates. The 
aggregate formation was mediated by ADP receptor activity, while the adhesion to collagen via GPVI led to exposure of PS, in agreement with previous observations at high platelet counts. ${ }^{90}$ The improved thrombus formation was of $57 \%$, predicted by the increase in platelet concentration, which is high given the inherent variability $(10 \%)$ of flow tests. Furthermore, in vitro reconstitution of pre-transfusion blood with the same platelet pool as used for transfusion resulted in an increase in thrombus formation that was similar to the increase seen in the post-perfusion blood. This provides a good indication that the measurement of thrombus formation is useful to determine the in vitro quality of platelets under conditions that are relevant for their in vivo function.

The measurements of thrombin generation in platelet-containing plasma demonstrated that the procoagulant response of the transfused platelets was preserved. After transfusion, the formation of thrombin in plasma was accelerated and this increase corresponded to the higher platelet count. As shown earlier for native platelets, ${ }^{102}$ transfused platelets are thus able to provide a procoagulant surface for enhancement of the thrombin-forming reaction. While assessing the responsiveness to ADP and collagen during thrombin generation, an average decreased response was found for transfused platelets that were previously stored in PAS-II medium. Earlier in vitro studies had indicated that the reactivity of platelets stored in synthetic medium decreased more rapidly than platelets stored in plasma. ${ }^{78,116}$ In the present test, the responses of these platelets varied considerably from patient to patient sample, which may result from differences between platelet batches or differences in recovery of the platelet preparations after transfusion. In vitro additions with stored platelets were not performed in thrombin generation experiments, because such platelet preparations all contain high concentrations of coagulation-active microparticles. ${ }^{100}$

Recently, we have shown that the diminished activation properties of stored platelets can partly be recovered in vitro in the presence of plasma or whole blood, by way of temperature-dependent activation of ADP-degrading enzymes. ${ }^{78,116}$ Such recovery of platelet activity is also likely to occur after transfusion in vivo. Evidence for this was separately obtained by flow cytometry, 
showing that agonist-induced integrin activation and P-selectin expression were increased in patient platelets collected after transfusion, in comparison with platelets from the same storage pool that were not transfused. The similarity in platelet responses after transfusion or reconstitution in flow-mediated adhesion suggests at first glance that recovery is similar in either case. However, in $40 \%$ of the cases, we noted that platelet aggregates were markedly larger in size after transfusion than in reconstituted blood samples. This difference can be due to either incomplete recovery in vitro or selective removal of platelet subpopulations from the circulation in vivo. Alternatively, it can be caused by differences in the patient conditions.

Under the present conditions of high shear rate, leucocytes did not interact with the thrombi formed on collagen. Leucocytes may contribute to thrombus formation in vivo, e.g. by interacting with platelets expressing P-selectin. In addition, leucocytes may participate in thrombin generation in vivo, for instance by expressing tissue factor. However, this remains speculative, because not much is known on the hemostatic function of leucocytes at low platelet concentrations, especially not in the present group of patients with reduced numbers of white blood cells after chemotherapy.

The cone-and-plate analyzer has been proposed for studies of improved platelet function after transfusion. ${ }^{253}$ This test also incorporates shear, but does not use laminar flow. Because it seems to lack sensitivity to $\alpha$ llb $\beta 3$ receptor function in comparison with the present method, ${ }^{253}$ we consider that a sensitive flow assay for bed-side use should be based on laminar flow. This could be achieved, for instance, by adaptations of the current platelet function analyzer (PFA-100), e.g. by altering capillary size and coatings and by providing timecourse readouts.

Among the patients of this study population, there were three atypical cases. One patient received amphotericin $B$ as antibiotic treatment prior to platelet transfusion. There are indications that this drug influences platelet properties upon transfusion. ${ }^{254-256}$ The present patient showed only a small increase in platelet concentration upon transfusion, which was accompanied by only minor increases in thrombus formation and thrombin generation. Apparently, the (few) 
circulating platelets were normally responsive. One other patient was without marked clinical symptoms, but flow experiments pointed to a remarkably high thrombus formation after transfusion, despite a relatively low post-transfusion platelet count. Thrombin generation in the plasma from this patient was not altered. The underlying cause of the high platelet responses under flow is unclear.

Furthermore, recurrent blood samples were analysed from one patient, who developed graft-versus-host disease. Even before transfusion, this patient repeatedly had high thrombin generation curves, which were not further increased by transfusion, and which contrasted to a normal thrombus formation. Graft-versus-host disease following stem cell transplantation can associate with increased coagulation and thrombosis risk, ${ }^{257}$ but also with bleeding complications. ${ }^{258}$ The current data indicate that, in this patient, thrombin generation in PRP and in PFP was increased. Also, the repeatedly high thrombin generation found in the absence of platelets (PFP samples) strongly points to the presence of (platelet-derived) microparticles in the plasma, as these provide the major coagulation-promoting surface in plasma without platelets. ${ }^{116}$

In summary, we conclude that measurements of thrombus formation under flow and of thrombin generation (platelet-dependent coagulation) can be used to determine the functional activity of platelets under thrombocytopenic conditions before and after transfusion. Furthermore, it appears that altered thrombus formation or coagulation at low platelet counts can be detected with these techniques. These tests seem to have increased sensitivity for measuring platelet function in thrombocytopenia than thromboelastography. In particular, the flow test is also useful for monitoring the functionality of concentrates of stored platelets, when platelets are allowed to resensitize before use. In contrast to the thrombin generation test, the flow test is as yet difficult to use as a bed-side assay, because it requires complex microscopic evaluation. Further adaptations to improve the detection technology are thus desired. 

Chapter 8

General discussion 
During aplastic thrombocytopenia or in case of low platelet counts due to bleeding, patients are transfused either prophylactically or therapeutically with donated platelets to ensure proper hemostasis. The purpose of the transfusion is to supply the patient with platelets that have similar hemostatic properties as autologous platelets. However, conditions of preparation and storage of platelets used for transfusion purposes affect their activity, because the cells are exposed to a non-physiological environment (e.g. the presence of anticoagulants, the absence of platelet inhibitors, storage in plastic containers with an artificial surface, and storage at room temperature). Both storage conditions and storage time are known to affect the hemostatic properties of platelets, which results in so-called 'platelet storage lesion'. In this thesis, we evaluated the changes in signal transduction of stored platelets in response to key agonists and the consequences of changed signaling for the platelet functions in thrombus formation, coagulation and eventually after transfusion. An overview of the main findings on alterations in platelet responses is depicted in figure 1.

\section{Preservation of platelet responses to purinergic nucleotides}

The purinergic compounds ADP and ATP are key agonists for platelet function. ${ }^{11,16,259,260}$ Isolated platelets slowly release these substances. This results in autocrine activation and subsequent desensitization of the purinergic receptors. ${ }^{115,156}$ Many laboratories use the ADP- and ATP-degrading enzyme apyrase to retain, even on a short time scale, the responses to these agonists in isolated platelet preparations. As ADP and ATP are also released during storage of platelets, ${ }^{261}$ it was expected that desensitization of the ADP and ATP receptors was one of the earlier storage-induced changes in platelets. In chapters 3 and 4 , we evaluated the signaling activity of the purinergic receptors $\left(P 2 Y_{1}, P 2 Y_{12}\right.$ and $\left.P 2 X_{1}\right)$, and found that centrifugation and wash of stored platelets greatly impaired their shape change reaction with ATP and aggregation response with ADP. However, it also appeared that these responses were only slightly diminished, as long as the platelets were kept in a $100 \%$ plasma environment. Storage of the cells in synthetic media, where 
plasma was (partially) replaced by a buffer solution, led to desensitization of the purinergic receptors. The unexpected preservation of purinergic receptor activity in plasma was due to the presence of plasma ecto-nucleotidases, capable of degrading ADP and ATP and, hence, antagonizing secretion-mediated purinergic receptor desensitization (figure $1 \mathrm{~A}$ ). By using specific inhibitors and assessing the nucleotide degradation profiles, we were able to identify two different ecto-nucleotidase activities in human plasma, i.e. the ecto-NTPDase CD39 and a different ecto-NPPase. Both ecto-nucleotidase activities are present on the surface of endothelial cells and circulating leukocytes. ${ }^{166,262}$ Moreover, both activities are considered to play a modulating role in thrombus formation after vascular injury. ${ }^{1,263}$ It is proposed that CD39 is also present on the surface of human platelets. ${ }^{170}$ However, the present results show that platelet-bound CD39 activity is low, and insufficient to antagonize the effects of released ADP and ATP during storage of platelets at low plasma concentrations. Others have reported that (cleaved) soluble forms of ectonucleotidases are present in whole blood and, at lower amounts, in purified plasma. ${ }^{264,265}$ These soluble enzymes, which are derived from endothelial and white cells, are likely to be responsible for the preservation of platelet function. We thus concluded that plasma contains a pool of ecto-nucleotidases that are continuously active during storage.

Currently, there is a tendency to store platelets in media with reduced plasma concentration, to reduce inflammatory reactions in the recipient and to save plasma for other purposes. ${ }^{266}$ However, the experiments in chapter 3 show that plasma helps to preserve purinergic receptor function during storage. In chapter 4 , we therefore observed this property in platelets that were stored in PAS-II medium where $70 \%$ of the plasma was replaced by synthetic buffer medium. Storage at low plasma content had two different effects: diminished responses of the platelets to ADP and ATP, and increased appearance at the cell surface of activation markers such as CD62. Likely, these platelets experience more autocrine activation, resulting in secretion and also in desensitization of the purinergic receptors. 
A

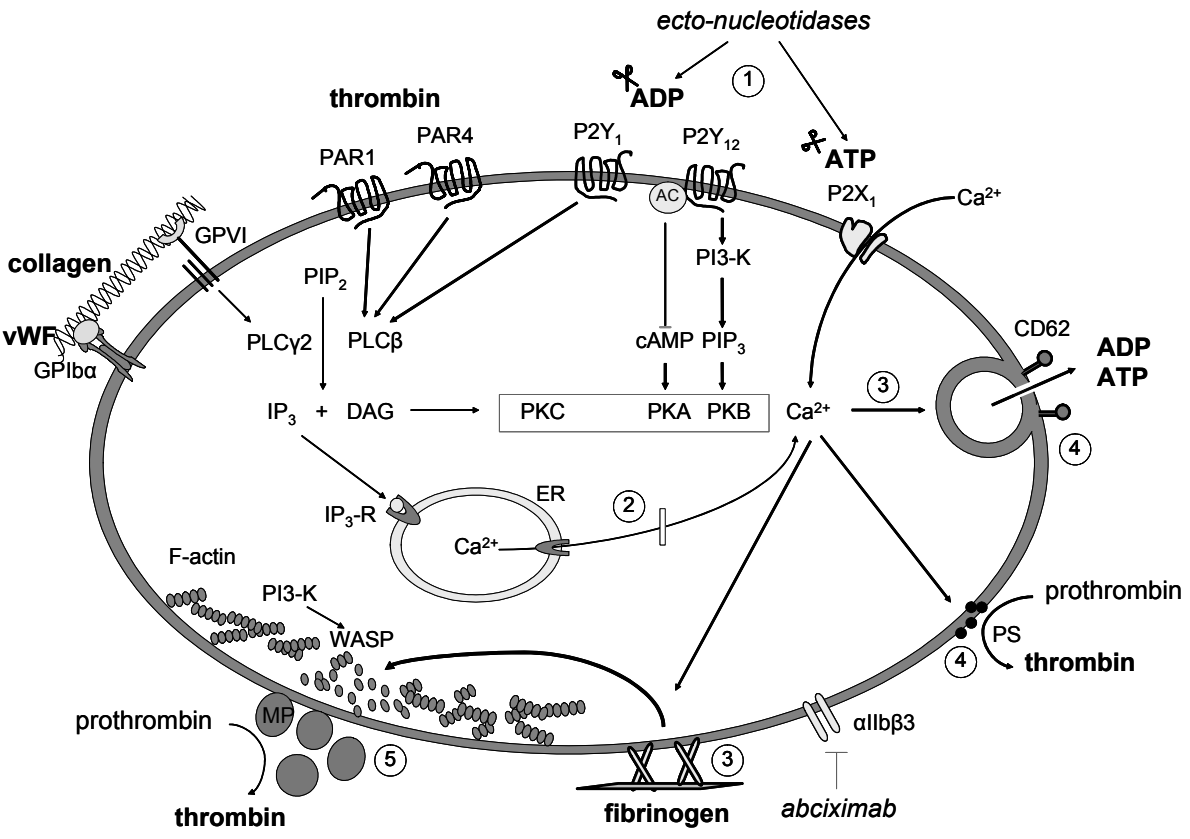

B

(1) ADP and ATP receptor function

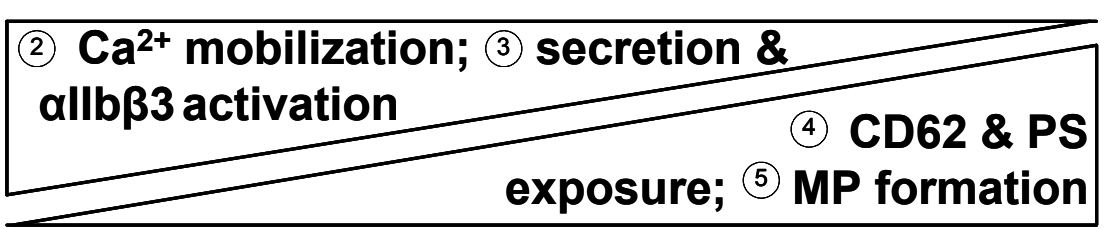

Figure 1. Overview of alterations in responsiveness and activation state of platelets during storage. Under normal conditions, resting platelets have a low activation state and are highly responsive to physiological agonists (indicated in bold); these include VWF, collagen, thrombin, ADP, ATP and fibrinogen. During activation, $\mathrm{Ca}^{2+}$ is mobilized by IP3 receptors in the endoplasmic reticulum (ER); and surface expression of activation markers increases, such as activated integrin allb33, CD62 (P-selectin) and PS. The protein kinases (PK), PI3-K, PKA, PKB and PKC are key mediators of the platelet activation process. For further explanation and abbreviations, see text. Panels $A$ and $B$ summarize the main storage-induced changes in activation properties of platelets, as discussed in this thesis:

1. desensitization of ADP and ATP receptors is prevented during storage by ecto-

nucleotidases, like CD39, present in plasma

2. stored platelets are less responsive, as less $\mathrm{Ca}^{2+}$ is mobilized from internal stores in response to agonists

3. in stored platelets, secretion and integrin $\alpha$ llb $\beta 3$ activation are impaired

4. stored platelets seem to have a higher activation state, since the surface expression of CD62

and PS increases

5 . storage is accompanied by allbß3-dependent shedding of microparticles (MP) by

rearrangement of F-actin via $\mathrm{PI} 3-\mathrm{K}$ and possibly WASP 
Others have shown that transfusion of platelets stored in PAS-II was found to be less effective in transfusion efficacy in comparison to that of platelets stored in full plasma, although the frequency of adverse post-transfusion side reactions had decreased in patients transfused with PAS-II platelets. ${ }^{77,181}$ This suggests that a larger fraction of the platelets stored in PAS-II medium (likely those with surface activation markers) are cleared from the circulation, but also that the transfused platelets may recover from purinergic receptor desensitization. In chapter 7, we used blood from thrombocytopenic patients transfused with platelets in plasma or PAS-II medium, to evaluate the ability of the platelets to form thrombi and support procoagulant activity. When adjusting for the posttransfusion platelet concentration, we detected only small differences in platelet function plasma and PAS-II stored platelets, indicating that the desensitization towards ADP and ATP of PAS-II stored platelets indeed is largely reversible.

In circulating blood, platelets are kept in an inactivated state by strong inhibitors, such as prostacyclin and nitric oxide, which are secreted by endothelial cells. ${ }^{267-269}$ These substances have a short half-life, meaning that they will not contribute to the preservation of platelets upon long-term storage in vitro. An interesting question is why the presence of relatively low amounts of soluble ecto-nucleotidases is sufficient to preserve platelet functions, in case of storage in the absence of strong platelet inhibitors. One answer is that stored platelets are not exposed to the strong agonist thrombin, due to the presence of anti-coagulant citrate in storage media. However, traces of thrombin are easily formed in the circulation, the effects of which can be annulled by prostacyclin and nitric oxide. Interestingly, it has been shown that infusion of nitric oxide into a platelet suspension, before processing and storage, decreased platelet storage lesion. ${ }^{138}$

\section{Alterations in platelet responsiveness during storage}

The results presented in this thesis support the understanding that the overall responsiveness of platelets decreases, while the surface exposure of activation markers increases with the time of storage. ${ }^{100}$ The latter change, as observed by others, ${ }^{123,261,270,271}$ is detected as a slow increase in $\alpha$ llb $\beta 3$ 
receptors with activated conformation, appearance of the secretion marker CD62 (P-selectin), and surface exposure of procoagulant phosphatidylserine (PS) (figure 1B). With respect to the decreased responsiveness, we explored the intracellular signaling pathways that may be involved in chapter 6 .

Mobilization of cytosolic $\mathrm{Ca}^{2+}$ is a common and early signaling step in the activation pathway of platelets upon activation by most physiological agonists. ${ }^{228}$ We thus measured the $\mathrm{Ca}^{2+}$ responses in stored platelets following stimulation with relevant agonists. These were compounds activating the tyrosine-kinase linked collagen receptor, glycoprotein VI (GPVI), which activates the phospholipase Cy2 (PLCY2) isoform; ${ }^{92}$ furthermore compounds activating the $G_{q}$ protein-coupled isoform phospholipase $C \beta(P L C \beta),{ }^{188}$ i.e. ADP, thromboxane $A_{2}$ and thrombin, which interact with the P2Y $1, T P \alpha$ and PAR1/4 receptors, respectively. Intriguingly, we found that storage of the cells led to a gradual decrease in $\mathrm{Ca}^{2+}$ responses induced by all agonists. This suggests that not primarily receptor-mediated activation of either PLCY2 or PLC $\beta$ is affected by storage time, but rather a step downstream of the production of inositol 1,4,5-trisphosphate (IP3), that controls the release of $\mathrm{Ca}^{2+}$ from the endoplasmic reticulum (figure $1 \mathrm{~A}$ ). We also found that the total content of the intracellular $\mathrm{Ca}^{2+}$ stores in the endoplasmic reticulum did not change. An interesting possibility for the hampered calcium response is that the IP3 receptor channel itself is affected by platelet storage.

Platelets express type I, II and III IP3 receptors, which operate as endomembrane $\mathrm{Ca}^{2+}$ channels, in a way modulated by the cytoplasmic levels of IP3, $\mathrm{Ca}^{2+}$ and ATP. ${ }^{228,272,273}$ The type-II receptors further control store-regulated $\mathrm{Ca}^{2+}$ influx in platelets. ${ }^{274}$ These IP3 channels are quite sensitive to both the activation status and metabolic conditions of the platelet. For instance, the channel activity is downregulated by cyclic-AMP dependent phosphorylation ${ }^{24}$ and altered by the redox state of glutathione in the platelets. ${ }^{234}$ Considering the gradual decrease in reduced glutathione and consequent changes in enzymatic activities, $^{236,275}$ it is conceivable that this type of change reduces the IP3 receptor function in stored platelets, but this has not yet been proven. 


\section{Integrin-dependent formation of microparticles during storage}

Early observations showed that (shear-stress) activated platelets shed microparticles (earlier termed as microvesicles) from their surface..$^{47,215,276,277}$ In platelets stimulated with $\mathrm{Ca}^{2+}$ ionophore or collagen/thrombin, the microparticle formation has been attributed to activation of the $\mathrm{Ca}^{2+}$-dependent protease calpain and subsequent degradation of actin-binding cytoskeletal

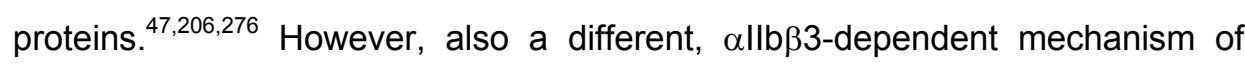
microparticle shedding may exist, acting independently of calpain. ${ }^{47}$ Platelet preparations stored at blood banks contain large amounts of procoagulant microparticles. ${ }^{100,278}$ These are supposed to result from inevitable activation during the isolation procedure of platelets.

In contrast, in chapter 5 we present evidence that microparticles can continuously be shed from stored platelets in the absence of activation. Various measurements indicated that this 'spontaneous' microparticle formation occurs at resting levels of cytosolic $\mathrm{Ca}^{2+}$ and at basal calpain activity. This shedding is the result of specific signaling events, namely of allbß3-dependent signal transduction via phosphoinositide 3-kinase (PI3-K) to the actin cytoskeleton (figure $1 \mathrm{~A}$ ). Further confirmation for outside-in signaling via allb $\beta 3$ integrins came from the finding that also allbß3-mediated spreading of platelets on fibrinogen - which is a process typically relying on outside-in signaling - led to formation of microparticles. Targeted modulation of this signal transduction, affecting proteins that link allb $\beta 3$ to the actin membrane skeleton, disrupted normal actin (dys)assembly and enhanced microparticle formation.

One can only speculate on the further intracellular events involved in integrin-dependent microparticle formation. A number of genetic disorders have been associated with aberrant microparticle formation. These are Glanzmann's thrombasthenia (absence of allbß3), the Scott syndrome (defective PS exposure) and the Wiscott-Aldrich syndrome (absence of actin-binding WASP). ${ }^{49,227,279}$ Clear indications for an involvement of WASP came from the finding that inhibition of WASP (using a cell-permeable inhibitor) potentiated the 
inhibitory effect of PI3-K on allbß3-dependent microparticle formation. ${ }^{280}$ Although more experimental work needs to be performed to support this finding, the concept is interesting since WASP is an important regulator of actin nucleation via the Arp2/3 complex. ${ }^{281}$ Thus, allbß3-induced activation of PI3-K and phosphorylation of WASP may control the actin filament formation and turnover that lead to local propulsions of the plasma membrane and later shedding of membrane vesicles.

Altogether, this research describes a new allbß33-dependent mechanism of microparticle formation, which acts in resting platelets, independently of $\mathrm{Ca}^{2+}$ elevation and calpain-induced degradation of the actin cytoskeleton. This mechanism is active in platelets stored under blood bank conditions even - or perhaps in particular - in the presence of plasma.

\section{Storage-dependent platelet surface changes and apoptosis}

During storage, platelets undergo several intracellular and surface changes, which often resemble those of activation, e.g. clustering of GPIb-V-IX and $\alpha$ llb $\beta 3$ and surface expression of CD62 and PS. ${ }^{86,100,282}$ Accordingly, the appearance of these epitopes on the platelet surface is considered to be a sign of platelet activation. It is however not clear whether these changes indeed reflect activation processes, i.e. mediated by specific signaling pathways, or may occur by a different way as in 'normal ageing'. Until quite recently, not much was known of the senescence process in platelets. It was assumed that ageing is associated with an apoptosis-like process, although the anucleated platelets lack several proteins of the 'normal' apoptotic machinery. Recent literature shows that the platelet life-time is controlled by the level of the killersuppressor protein, Bcl- $\mathrm{x}_{\mathrm{L}}$, which antagonizes the killer protein Bak and, hence, controls an apoptotic-like process that is still not well understood. ${ }^{283}$ This finding sheds new light on earlier papers proposing that platelet storage leads to activation of cell death proteins, which mediate the surface exposure of PS and the clearing of non-viable platelets after transfusion. ${ }^{179,284,285}$ It is likely that storage-induced changes in cytosolic $\mathrm{Ca}^{2+}$ concentration, as demonstrated in this thesis, are involved in these apoptotic-like events, but how is still unclear. 
Other events in stored platelets that are reminiscent to apoptosis are the surface exposure of PS and the shedding of microparticles. Furthermore, during storage intracellular proteins can be cleaved such as focal adhesion kinase, Src kinase and gelsolin. In this case, it is not clear whether the proteolysis is mediated by activated caspase due to ageing or by activated calpain due to slow activation. ${ }^{111,112}$ In our experiments, the activity of calpain remained low during storage (chapter 5 ). This suggests that this enzyme plays a minor role in regulating the platelet life-time.

After transfusion, platelets and microparticles that express CD62 or PS are rapidly cleared from the circulation by monocytes and macrophages. ${ }^{286}$ This clearance resembles the normal scavenging of other types of apoptotic (PSexpressing) cells. Also other surface changes on stored platelets can provoke clearance after transfusion. These include clusters of the GPIb-V-IX complex, which are formed whithout platelet activation and are recognized by the scavenging Mac1 receptor on macrophages. ${ }^{86,144,287}$ Furthermore, many glycoproteins e.g. GPIba, GPV, GPVI and CD62 appear to be cleaved from the surface of platelets during storage. ${ }^{103,105,121,271,288}$ Recently, platelet-derived metalloproteinases (ADAM 10 and 17) have been discovered, which can mediate this cleavage. ${ }^{104,289}$ How glycoprotein cleavage precisely relates to the hemostatic function of platelets and to clearance is still not known. In summary, it seems that (old) platelets are removed from the circulation due to surface changes caused by both apoptotic-like and activation-mediated processes, which may partially overlap. In our view, attempts to extend the shelf life-time of platelets for transfusion purposes need to focus on preservation of high responsiveness, and not only on minimizing the surface changes that provoke scavenging.

\section{Assessment of the hemostatic function of stored platelets}

At present, blood banks assess platelet functionality routinely by checking the metabolic state of the cells. Thus, in stored platelet preparations, $\mathrm{pH}$ and glucose levels are usually measured, as well as the blood gasses $\mathrm{CO}_{2}$ and $\mathrm{O}_{2}$. However, measurements of the mitochondrial membrane potential show that 
the metabolic state (ATP level) of platelets remains high even upon prolonged storage. $^{238}$ Accordingly, changes in metabolic parameters will only provide information on advanced stages of deterioration of the platelets. Another routine test at blood banks, is the so-called 'swirl' test which, by visual inspection, assesses whether suspensions of platelets have maintained their discoid shape. This test likely senses the resting state of the cells, but it does not measure responsiveness to agonists. The findings in this thesis indicate that stored platelets can preserve integrity, but still loose their ability to respond to physiological agonists. In other words, to measure the role of platelets in hemostasis, it is important to measure relevant responses, like $\mathrm{Ca}^{2+}$ signal generation, aggregation and procoagulant activity. An important consideration in this respect is that such function assays should not be confined to the isolated platelet preparation, but also extended after transfusion.

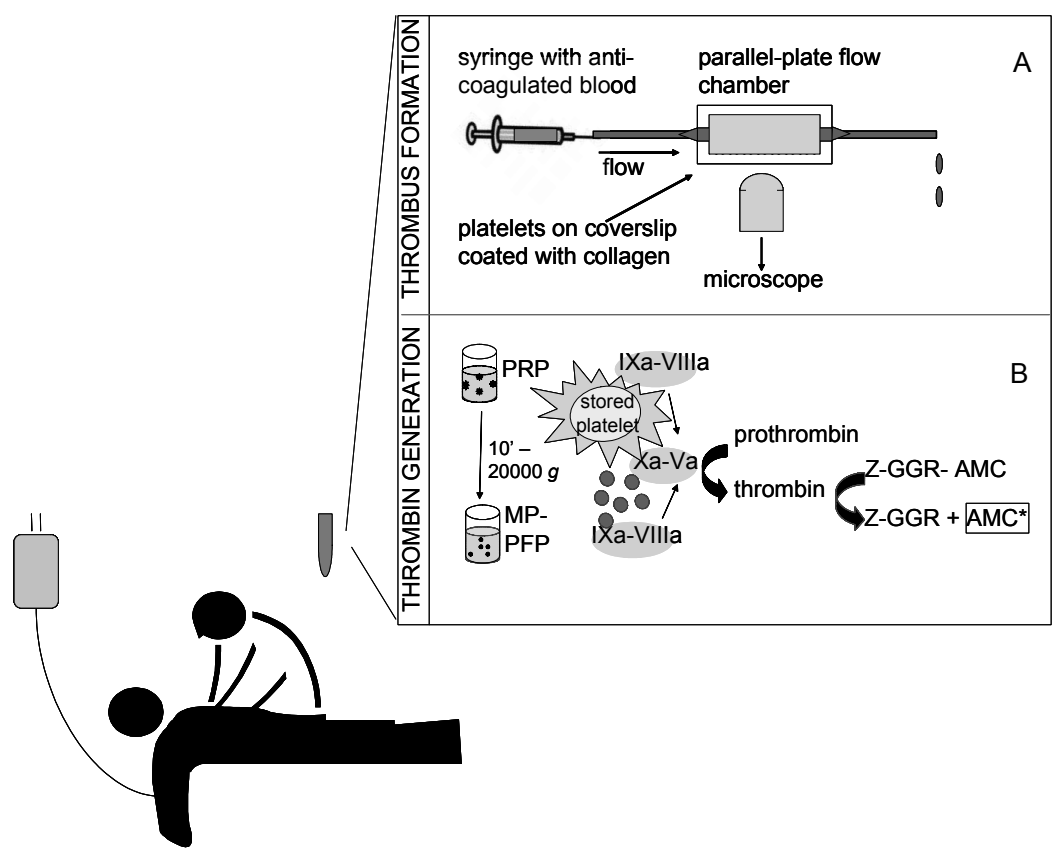

Figure 2. Tests to evaluate the hemostatic activity of stored platelets in thrombocytopenia after transfusion. Overview of two assays described in this thesis. A. Collagen-induced thrombus formation in whole blood under flow. Blood from thrombocytopenic patients after transfusion is perfused over a collagen surface at defined shear rate, and platelet deposition is measured. B. Platelet-rich plasma (PRP) from thrombocytopenic patients is used, or is centrifuged to prepare microparticle-containing platelet-free plasma (MP-PFP). Both preparations are used to measure thrombin generation, after triggering with tissue factor and $\mathrm{CaCl}_{2}$. Procoagulant surface from activated platelets or MP supports the activation of coagulation factors, which is measured after cleavage of the fluorescent thrombin substrate, Z-GGR-AMC. For further details, see text. 
Because most platelet function tests cannot be used at low platelet counts, we developed new assays in which platelet activity can be measured under conditions of thrombocytopenia, making use of recently developed technologies. ${ }^{212,232}$ In particular, these tests should overcome the limitations of flow cytometry, which technique well evaluates the ability of platelets to expose CD62 and activated $\alpha$ llb $\beta 3$, but not their interaction with adhesive substrates or other cells. In chapter 7, two such tests that cover primary (thrombus formation) and secondary (coagulation) hemostasis are described. In the first test, wholeblood thrombus formation is evaluated from the ability of transfused platelets to adhere and aggregate at high shear-rate to a collagen surface, such as present in damaged vascular endothelium (figure 2). The second test assesses the ability of transfused platelets to expose PS and to promote tissue factor-induced thrombin generation and, hence, coagulation. In both tests, an increase in platelet count correlated with increased platelet function, measured as either platelet deposition on collagen or rate of thrombin generation. Importantly, both tests also reported of an increase in platelet function in post-transfusion blood samples in comparison to pre-transfusion samples. Furthermore, experiments with ADP receptor blockers showed that, in either test, the platelets in posttransfusion blood were able to secrete ADP and to respond to this autocrine agent. Accordingly, we concluded that the tests provide relevant and additive information on the hemostatic activity of patient blood after a transfusion.

Interestingly, these tests also pointed to apparent abnormalities in specific patients. The post-transfusion blood of a patient using amphotericin B showed only low deposition of platelets on collagen. In the pre- and post-transfusion blood samples of a few other patients, substantial thrombin generation was measured not only in platelet-rich plasma, but also in platelet-free plasma fractions, which was indicative of the presence of microparticles. This was particularly clear in a patient, who had developed graft-versus-host disease. Since all patients receive microparticles together with platelets upon transfusion, the microparticle-related thrombin generation might be directly derived from the donor preparation. Alternatively, the microparticles might be due to post-transfusion activation or destruction of the transfused platelets or 
possibly derived from endothelial cells due to drug-induced vascular damage. Note that all post-transfusion samples were obtained at about 1 hour after the transfusion intervention. These and other observations present evidence that the developed tests can provide novel information on the hemostatic condition of a thrombocytopenic patient following transfusion.

\section{Relevance for blood bank practice}

To achieve optimal hemostasis, platelets should be highly responsive to their physiological agonists; adequately participate in adhesion, thrombus formation and coagulation; and remain in the circulation for a long time. The same characteristics are desired for platelets that are used for transfusion to patients with impaired hemostasis as in thrombocytopenia. At blood banks, many efforts are currently made to improve the quality of platelet preparations, particularly by improving the storage conditions. ${ }^{69}$ As argued in this thesis, the testing of such platelet preparations should not be limited to measurement of metabolic parameters, since these will only provide information on drastic changes related to vitality. Instead, it is important to choose function assays that inform on the ability of platelets to respond to their natural agonists. For instance, we showed that platelets stored in synthetic medium (PAS-II) loose their ability to respond to ADP and ATP, which are key agonists mediating platelet aggregation and thrombus formation. On the other hand, such platelets were not impaired in metabolic parameters. ${ }^{181,290,291}$ This illustrates that quality tests should include measurements of platelet responsiveness as well. Newly developed functional assays that can be used to test platelet activity in thrombocytopenia are described in chapter 7 . Note that shear-dependent thrombus formation can also be measured with the commercial PFA-100 system, but this device needs to be adapted for conditions with lower platelet counts in order to be useful for monitoring the effect of a transfusion. ${ }^{292,293}$ It should also be emphasized that the surface expression of so-called activation markers, such as CD62, does not necessarily point to a diminished responsiveness of the platelets. These activation makers, however, will likely result in increased clearance of platelets after a transfusion. ${ }^{86,179,287}$ Accordingly, it is important to use a test system that 
informs on both the responsiveness of the platelets and the development of activation markers during storage.

This thesis provides information for the further improvement of the storage conditions. Given the identified role of the plasma ecto-nucleotidase, CD39, in protection towards adenosine nucleotide receptor desensitization, it should be considered to supplement ADP-degrading substances to platelets stored in synthetic media. Also, supplementation of cyclic-AMP elevating agents (prostacyclin, adenosine) could be beneficial for keeping highly responsive platelets. Hopefully, the plea of this thesis to assess responsiveness of platelets leads to better testing of platelets stored at blood banks and, in the end, to improved platelet transfusion therapy.

\section{Implications for platelet transfusion therapy}

Before 1960 platelet transfusions were not being used and death from bleeding complications was common in leukemic patients receiving intensive chemotherapy. ${ }^{294}$ Still at present days, good surrogate endpoints to determine the hemostatic effect of a prophylactic platelet transfusion are still missing. One parameter widely used is the corrected count increment $(\mathrm{CCl})$, which informs on the increase in platelet count after transfusion of a certain number of platelets, corrected for the body surface area of the patient. Current guidelines for transfusion of one batch of platelets are that, at 1 and 24 hours after transfusion, the $\mathrm{CCl}$ should be $>7.5$ and $>2.5$, respectively. ${ }^{68}$ However, by definition, this parameter measures the quantitative efficacy of the transfusion intervention (i.e. the fraction of still circulating platelets), rather than the hemostatic effect of the intervention.

It is remarkable that different countries still use different types of platelet preparations for transfusion. ${ }^{69,295,296}$ In the Netherlands and other European countries, the so-called pooled buffy-coat platelet-plasma preparations are used as a standard and, in certain regions, part of the plasma is replaced by synthetic medium. In the United States of America, however, plasma-apheresis platelets are the standard preparations for transfusion, while synthetic media are not being used. ${ }^{297}$ Extensive comparisons between these platelet preparations in 
terms of responsiveness have not been made, neither in vitro nor in vivo in post-transfusion blood. New synthetic media with supplemented $\mathrm{Mg}^{2+}$ and $\mathrm{K}^{+}$ ions are under development, and these may give improved ways of storage, provided that autocrine/desensitization effects are suppressed as well. ${ }^{176,298,299}$ In summary, the removal of platelets from their natural environment necessarily influences their reactivity. It is therefore clear that storage conditions need to be optimized, and that functional assays monitoring post-transfusion platelet survival and function are required.

A recurrent question is whether transfusions with platelets may be replaced by infusion of procoagulant platelet membranes or even phospholipids. ${ }^{300,301}$ In our view, this question strongly depends on whether patients need direct hemostatic support to stop bleeding or prolonged support to reduce the risk of bleeding. Patients that require therapeutic platelet transfusions could benefit from highly procoagulant membranes, as these will acutely mediate the formation of thrombin and fibrin clots. The immediate risk, however, is venous and peripheral thrombosis. Furthermore, the role of such microparticles in mediating cellular-cross talk, leading to the recruitment of e.g. leukocytes and therefore increased inflammation, is still under intensive investigation. ${ }^{14,302}$ In the future, therapeutic platelet transfusion therapy could shift to this direction, but one should always take into account the possibility of thrombotic or inflammatory complications. Non-bleeding thrombocytopenic patients, on the other hand, will mostly benefit from an intervention causing long term protection against bleeding. Since we and others have shown that procoagulant membranes (microparticles) are rapidly cleared, it is doubtful whether these microparticles may play a beneficial role in prophylactic hemostatic treatment. ${ }^{286}$ Current practice of utilizing prophylactic platelet transfusions is not based on rigorous randomized studies and may be suboptimal or even unnecessary. There are relatively little data on platelet count and bleeding frequency available in patients with thrombocytopenia related to the bone marrow failure state. Furthermore, the daily platelet consumption of about $20 \times 10^{9} / \mathrm{L}$ to maintain endothelial integrity is increased when patients undergo (non)inflammatory reactions, like fever or infection. ${ }^{77,303}$ Therefore we need the validation of 
improved hemostatic assays, possibly including the measurement of endothelial markers, that can better determine the bleeding risk in patients with thrombocytopenia. This will then lead to better transfusion therapy policy (likely reducing the number of transfusions given) and will also help the blood bank in defining what a good platelet product should consist of. In this thesis, we therefore hope to have clearly described the mechanisms of storage-induced platelet storage lesion and the consequence of this on platelet transfusion therapy. 

Samenvatting 
Bloedplaatjes zijn cellulaire fragmenten, die afgesplitst worden door de megakaryocyten in het beenmerg, en die een cruciale rol spelen in het hemostatische proces. Bij vaatwandbeschadiging bewerkstelligen zij de stelping van het bloed door samen te klonteren en dan de stolling mogelijk te maken. Een sterk verlaagd aantal plaatjes in de circulatie, bijvoorbeeld door een afwijkende beenmergfunctie of een verhoogd verbruik of verlies aan plaatjes, leidt tot onvoldoende hemostase en versterkt daarmee het risico op levensbedreigende bloedingen. In dergelijke situaties worden patiënten getransfundeerd met een plaatjespreparaat dat afkomstig is van bloeddonoren. Deze donorplaatjes dienen geruime tijd na de transfusie in de circulatie te blijven. Meetal wordt aangenomen is dat een verhoging van het aantal circulerende plaatjes vanzelf leidt tot een verbeterde hemostase in de patiënt. Tot op heden is er nauwelijks ingegaan op de vraag of de plaatjes die getransfundeerd worden voldoende hemostatisch actief zijn. Deze vraag is niet onbelangrijk, omdat het risico op bloedingen enkel zal verkleinen wanneer de donorplaatjes, net als de autologe plaatjes, in staat zijn om adequaat te reageren op een vaatwandbeschadiging.

Zoals beschreven in hoofdstuk 1 , definiëren wij het reactievermogen van plaatjes als de capaciteit om te kunnen reageren op belangrijke fysiologische stimuli. Hieronder vallen collageen en de von Willebrand factor, die na een vaatwandbeschadiging beide blootgesteld worden aan de bloedstroom; verder de plaatjesproducten ADP, ATP en tromboxaan $A_{2}$, welke autocrien gesecreteerd worden; fibrinogeen waarmee geactiveerde plaatjes aan elkaar kunnen kleven; en ook trombine dat het meest actieve product is van de bloedstolling. Wanneer een bloedvat beschadigd wordt, leidt dit eerst tot een stabiele adhesie van plaatjes, vervolgens tot activeringsreacties via een verhoging van de vrije calciumconcentratie in het cytosol, en vormverandering van de plaatjes. Vervolgens verkleven de plaatjes tot een aggregaat (trombus), en onder invloed van trombine ontstaat er een stevig stolsel van plaatjes en fibrine. Als gevolg van deze plaatjesreacties wordt de beschadiging in het vat efficiënt gerepareerd en daarmee de bloeding gestopt. 
Hoofdstuk 2 geeft een overzicht van de literatuur die beschrijt dat de opslag van donorplaatjes, zoals die plaatsvindt bij de bloedbank, leidt tot veranderingen in hun functionaliteit. Deze veranderingen die gepaard gaan met opslag worden vaak omschreven als platelet storage lesion. Aangenomen wordt dat deze lesie beïnvloed wordt door de wijze van verwerking van het donorbloed en de manier van opslag van de cellen. Het begrip platelet storage lesion blijkt echter niet eenduidig gedefinieerd te zijn, maar sterk afhankelijk van de test waarmee de functionaliteit van de opgeslagen plaatjes bepaald wordt. De meest gebruikelijke testen betreffen het energiemetabolisme van de plaatjes, hun integriteit dan wel het voorkomen van activatiemerkers op het celoppervlak. Een nadeel is dat dergelijke assays ongevoelig zijn voor het opsporen van veranderingen in het reactievermogen van plaatjes, die met fysiologische agonisten gestimuleerd worden. In dit proefschrift is daarom gezocht naar meer gevoelige technieken om deze veranderingen te bepalen, zowel voor als na de transfusie van plaatjes in een patiënt.

Bekend was dat plaatjes tijdens hun bereiding en opslag kleine hoeveelheden ADP en ATP secreteren, als gevolg waarvan de plaatjesreceptoren voor ADP en ATP zeer snel geïnactiveerd raken. Het was dus te verwachten dat één van de eerste effecten van plaatjesopslag het verlies van reactiviteit op ADP en ATP zou zijn. Deze veronderstelling bleek niet zonder meer te kloppen. Hoofdstuk 3 beschrijft dat de P2Y-receptoren voor ADP en de P2X ${ }_{1}$-receptoren voor ATP lange tijd actief blijven, wanneer de plaatjes opgeslagen worden in plasma. Daarmee wordt ook het vermogen van de cellen tot vormverandering en aggregatie behouden. Aangetoond is dat het plasma specifieke enzymen bevat, zogenaamde ecto-nucleotidasen, die instaan voor de afbraak van gesecreteerd ADP en ATP. Desensitizatie van de ADP- en ATP-receptoren door autocriene secretie blijkt dus van minder belang, zolang plaatjes opgeslagen worden in hun natuurlijk medium, namelijk plasma.

Om kostenbesparende en andere redenen is er een tendens om plaatjes op te slaan in buffermedia (synthetische bewaarvloeistoffen) met slechts een beperkte hoeveelheid plasma. Hoofdstuk 4 beschrijft de functionaliteit van plaatjes die opgeslagen worden in dergelijk buffermedia. Deze plaatjes 
vertonen in vitro een verhoogde mate van activering (meer expressie van $\mathrm{P}$ selectine) en een verminderd reactievermogen op ADP, ten gevolge van ADPreceptor desensitizatie. Het verlagen van de hoeveelheid plasma, en daarmee de hoeveelheid ADP-afbrekende enzymen in de bewaarvloeistof, leidt dus tot een verslechterde conditie van de plaatjes tijdens opslag. Een gevolg hiervan is een verminderde ADP receptorsignalering tijdens trombusvorming onder stromingscondities.

Een typisch fenomeen dat optreedt tijdens de opslag van plaatjes is het afsnoeren van stolactieve micropartikels. In hoofdstuk 5 is bestudeerd in hoeverre deze micropartikelvorming het gevolg is van een verhoogde activatietoestand van de fibrinogeenreceptor (integrine $\alpha$ llb $\beta 3$ ). Aangetoond is dat geactiveerd $\alpha$ llb $\beta 3$ op de plaatjes een signaal initieert, dat leidt tot een lokaal verhoogde afbraak van filamenteus actine, en daarmee tot het loskomen van celskelet en plasmamembraan. Het gevolg van deze membraandestabilizatie is de vorming van micropartikels, die door een verlies van membraan-asymmetrie stolactief worden. Dit mechanisme verschilt wezenlijk van de eerder beschreven vorming van micropartikels door plaatjes die gestimuleerd worden met sterke agonisten zoals trombine en collageen. In dit laatste geval ontstaan de micropartikels na een verhoging in cytosolisch calcium en activatie van calpaïne, dat op zijn beurt instaat voor afbraak van het celskelet. In tegenstelling hiermee kon geen functie van calpaïne detecteerd worden in micropartikels die afgesnoerd werden door opgeslagen plaatjes. In hoeverre deze stolactieve micropartikels na transfusie bijdragen tot het stoppen van een bloeding is nog onduidelijk. Wel staat vast dat zij snel uit de circulatie verdwijnen.

Plaatjes opgeslagen in plasma bleken in verminderde mate in staat te zijn tot trombusvorming in vergelijking tot vers geïsoleerde plaatjes. Aangezien het verminderde reactievermogen van de opgeslagen plaatjes niet het gevolg was van desensitizatie van de ADP- en ATP-receptoren, is in hoofdstuk 6 gezocht naar alternatieve verklaringen. Plaatjesopslag leidde tot een verminderd vermogen tot calciummobilizatie vanuit het endoplasmatisch reticulum naar het cytosol. Deze verlaagde calciumrespons was onafhankelijk van het type agonist 
en van de signaalweg gebruikt door de agonisten. Aangezien de calciumvoorraad in het endoplasmatisch reticulum niet verminderde na opslag van plaatjes, werd de verminderde calciummobilizatie verklaard door een verminderde werking van de inositol 1,4,5-trifosfaat-receptoren, die als calciumkanalen fungeren. Er zijn aanwijzingen dat bij veroudering een verminderde calciummobilizatie de voorbode is van een apoptose-achtig proces, en daarmee tot een verkorting van de circulatietijd van de plaatjes. Dit biedt een nieuwe uitdaging voor de bloedbank, namelijk zoeken naar middelen om de veranderde calciummobilizatie en apoptose te vertragen om daarmee de maximale opslagtijd van plaatjes (nu 5 tot 7 dagen) te verlengen.

In hoofdstuk 7 is het verlaagde reactievermogen als gevolg van plaatjesopslag bestudeerd na transfusie in patiënten met lage plaatjesaantallen. Dit betrof leukemiepatiënten, die trombocytopeen waren ten gevolge van de behandeling voor deze aandoening. Aangezien er geen goede methoden beschikbaar waren voor het testen van de plaatjesfunctie in plasma of bloed van patiënten met trombocytopenie, moesten deze eerst ontwikkeld worden. Hoofdstuk 7 beschrijft twee van zulke testen, namelijk meting van de trombusvorming in bloed onder stromingscondities, en meting van de trombinegeneratie in stollend plaatjes-rijk plasma. Bij een groep patiënten werd nagegaan dat deze testen inderdaad in staat zijn om positieve effecten van een transfusie met plaatjes te detecteren. Aangetoond werd dat opgeslagen plaatjes na transfusie goed in staat zijn trombi te vormen en de generatie van trombine te stimuleren.

Deze twee testen zijn verder gebruikt om na transfusie de functionaliteit te bepalen van plaatjes, die opgeslagen zijn in plasma of in synthetische bewaarvloeistof. In het laatste geval kon een herstel in reactievermogen op ADP geconstateerd worden. Verder bleken beide functietesten nuttig te zijn voor het opsporen van afwijkingen in trombusvorming of stolling, bijvoorbeeld bij een patiënt met graft-versus-host disease. Verwacht wordt dat deze testen in de toekomst bruikbaar zullen zijn voor het bepalen van de hemostase-activiteit in bloed van patiënten met trombocytopenie, en dan als indicator kunnen dienen voor het risico op bloedingen. 
De algemene discussie in hoofstuk 8 bespreekt de bevindingen van dit proefschrift in verhouding tot de recente literatuur. In het bijzonder wordt ingegaan op de veranderingen in signalering die optreden bij de opslag van plaatjes, en op de gevolgen hiervan voor de trombusvorming en het op gang brengen van de stolling. Geconcludeerd is dat in vivo studies van belang zijn om een goed beeld te krijgen van het precieze hemostatisch effect van een plaatjestransfusie. Al met al dragen de studies beschreven in dit proefschrift bij tot een beter begrip van het proces van platelet storage lesion en de mogelijke consequenties daarvan voor de transfusiepraktijk. De beschreven resultaten helpen hopelijk om de bewaarcondities van plaatjes te optimaliseren en de praktijk rond plaatjestransfusie te verbeteren. 
References 
1. Marcus AJ, Broekman MJ, Drosopoulos JH, Olson KE, Islam N, Pinsky DJ, Levi R. Role of CD39 (NTPDase-1) in thromboregulation, cerebroprotection, and cardioprotection. Semin Thromb Hemost 2005; 31:234-46.

2. Patel SR, Hartwig JH, Italiano JE, Jr. The biogenesis of platelets from megakaryocyte proplatelets. J Clin Invest 2005; 115:3348-54.

3. Stuart MJ, Murphy S, Oski FA. A simple non-radioisotope technic for the determination of platelet life-span. N Engl J Med 1975; 292:1310-3.

4. Gibbins JM. Platelet adhesion signaling and the regulation of thrombus formation. J Cell Sci 2004; 117:3415-25.

5. Heemskerk JW, Kuijpers MJ, Munnix IC, Siljander PR. Platelet collagen receptors and coagulation. A characteristic platelet response as possible target for anti-thrombotic treatment. Trends Cardiovasc Med 2005; 15:86-92.

6. Nieswandt B, Aktas B, Moers A, Sachs UJ. Platelets in atherothrombosis: lessons from mouse models. J Thromb Haemost 2005; 3:1725-36.

7. Mendolicchio GL, Ruggeri ZM. New perspectives on von Willebrand factor functions in hemostasis and thrombosis. Semin Hematol 2005; 42:5-14.

8. de Groot PG. The role of von Willebrand factor in platelet function. Semin Thromb Hemost 2002; 28:133-8.

9. Auger JM, Kuijpers MJ, Senis YA, Watson SP, Heemskerk JW. Adhesion of human and mouse platelets to collagen under shear: a unifying model. Faseb J 2005; 19:825-7.

10. Farndale RW, Sixma JJ, Barnes MJ, de Groot PG. The role of collagen in thrombosis and hemostasis. J Thromb Haemost 2004; 2:561-73.

11. Kahner BN, Shankar H, Murugappan S, Prasad GL, Kunapuli SP. Nucleotide receptor signaling in platelets. J Thromb Haemost 2006; 4:2317-26.

12. Jackson SP, Nesbitt WS, Kulkarni S. Signaling events underlying thrombus formation. $J$ Thromb Haemost 2003; 1:1602-12.

13. Payrastre B, Missy K, Trumel C, Bodin S, Plantavid M, Chap H. The integrin allb $\beta 3$ in human platelet signal transduction. Biochem Pharmacol 2000; 60:1069-74.

14. Morel O, Toti F, Hugel B, Bakouboula B, Camoin-Jau L, Dignat-George F, Freyssinet JM. Procoagulant microparticles: disrupting the vascular homeostasis equation? Arterioscler Thromb Vasc Biol 2006; 26:2594-604.

15. Gachet C, Hechler B. The platelet P2 receptors in thrombosis. Semin Thromb Hemost 2005 31:162-7.

16. van Gestel MA, Heemskerk JW, Slaaf DW, Heijnen VV, Reneman RS, oude Egbrink MG. In vivo blockade of platelet $A D P$ receptor $\mathrm{P}_{2} \mathrm{Y}_{12}$ reduces embolus and thrombus formation but not thrombus stability. Arterioscler Thromb Vasc Biol 2003; 23:518-23.

17. Bauer M, Retzer M, Wilde JI, Maschberger P, Essler M, Aepfelbacher M, Watson SP, Siess $W$. Dichotomous regulation of myosin phosphorylation and shape change by Rho-kinase and $\mathrm{Ca}^{2+}$ in intact human platelets. Blood 1999; 94:1665-72.

18. Oury C, Toth-Zsamboki E, Vermylen J, Hoylaerts MF. P2X $\mathrm{X}_{1}$-mediated activation of extracellular signal-regulated kinase 2 contributes to platelet secretion and aggregation induced by collagen. Blood 2002; 100:2499-505.

19. Erhardt JA, Toomey JR, Douglas SA, Johns DG. P2X stimulation promotes thrombin receptor-mediated platelet aggregation. J Thromb Haemost 2006; 4:882-90.

20. Berridge MJ. Inositol trisphosphate and $\mathrm{Ca}^{2+}$ signaling. Nature 1993; 361:315-25.

21. Heemskerk JW, Feijge MA, Sage SO, Walter U. Indirect regulation of $\mathrm{Ca}^{2+}$ entry by cAMPdependent and cGMP-dependent protein kinases and phospholipase $\mathrm{C}$ in rat platelets. Eur J 
Biochem 1994; 223:543-51.

22. Werner $\mathrm{MH}$, Hannun YA. Delayed accumulation of diacylglycerol in platelets as a mechanism for regulation of onset of aggregation and secretion. Blood 1991; 78:435-44.

23. Brass LF, Woolkalis MJ, Manning DR. Interactions in platelets between G proteins and the agonists that stimulate phospholipase $C$ and inhibit adenylyl cyclase. J Biol Chem 1988; 263:5348-55.

24. Keularts IM, van Gorp RM, Feijge MA, Vuist WM, Heemskerk JW. $\alpha 2 A$-adrenergic receptor stimulation potentiates $\mathrm{Ca}^{2+}$ release in platelets by modulating cAMP levels. J Biol Chem 2000; 275:1763-72.

25. Jackson SP, Schoenwaelder SM, Goncalves I, Nesbitt WS, Yap CL, Wright CE, Kenche V, Anderson KE, Dopheide SM, Yuan Y, Sturgeon SA, Prabaharan H, Thompson PE, Smith GD, Shepherd PR, Daniele N, Kulkarni S, Abbott B, Saylik D, Jones C, Lu L, Giuliano S, Hughan SC, Angus JA, Robertson AD, Salem HH. PI 3-kinase p110ß: a new target for antithrombotic therapy. Nat Med 2005; 11:507-14.

26. Dangelmaier $C$, Jin J, Smith JB, Kunapuli SP. Potentiation of thromboxane $A_{2}$-induced platelet secretion by $G_{i}$ signaling through the phosphoinositide-3 kinase pathway. Thromb Haemost 2001; 85:341-8.

27. Cosemans JM, Munnix IC, Wetzker R, Heller R, Jackson SP, Heemskerk JW. Continuous signaling via PI3K isoforms $\beta$ and $\gamma$ is required for platelet ADP receptor function in dynamic thrombus stabilization. Blood 2006; 108:3045-52.

28. Hirsch E, Bosco O, Tropel P, Laffargue M, Calvez R, Altruda F, Wymann M, Montrucchio G. Resistance to thromboembolism in PI3K $\gamma$-deficient mice. Faseb J 2001; 15:2019-21.

29. Woulfe D, Jiang H, Morgans A, Monks R, Birnbaum M, Brass LF. Defects in secretion, aggregation and thrombus formation in platelets from mice lacking Akt2. J Clin Invest 2004; 113:441-50.

30. Wong DA, Kita Y, Uozumi N, Shimizu T. Discrete role for cytosolic phospholipase $A_{2} \alpha$ in platelets: studies using single and double mutant mice of cytosolic and group IIA secretory phospholipase $A_{2}$. J Exp Med 2002; 196:349-57.

31. Caughey GE, Cleland LG, Gamble JR, James MJ. Up-regulation of endothelial cyclooxygenase-2 and prostanoid synthesis by platelets. Role of thromboxane $A_{2}$. J Biol Chem $2001 ; 276: 37839-45$

32. Quinton TM, Murugappan S, Kim S, Jin J, Kunapuli SP. Different G protein-coupled signaling pathways are involved in a granule release from human platelets. J Thromb Haemost 2004; 2:978-84.

33. Paul BZ, Jin J, Kunapuli SP. Molecular mechanism of thromboxane $A_{2}$-induced platelet aggregation. Essential role for P2t $\mathrm{t}_{\mathrm{ac}}$ and $\alpha_{2 \mathrm{a}}$ receptors. J Biol Chem 1999; 274:29108-14.

34. Shattil SJ, Kashiwagi H, Pampori N. Integrin signaling: the platelet paradigm. Blood 1998; 91:2645-57.

35. Goto S, Tamura N, Ishida H, Ruggeri ZM. Dependence of platelet thrombus stability on sustained GPIlb/llla activation through adenosine 5'-diphosphate receptor stimulation and cyclic $\mathrm{Ca}^{2+}$ signaling. J Am Coll Cardiol 2006; 47:155-62.

36. Hato T, Pampori N, Shattil SJ. Complementary roles for receptor clustering and conformational change in the adhesive and signaling functions of integrin $\alpha$ llb $\beta 3$. J Cell Biol 1998; 141:1685-95.

37. Shattil SJ, Newman PJ. Integrins: dynamic scaffolds for adhesion and signaling in platelets. Blood 2004; 104:1606-15.

38. Arias-Salgado EG, Lizano S, Sarkar S, Brugge JS, Ginsberg MH, Shattil SJ. Src kinase activation by direct interaction with the integrin $\beta$ cytoplasmic domain. Proc Natl Acad Sci U S A 2003; 100:13298-302. 
39. Saci A, Rendu F, Bachelot-Loza C. Platelet $\alpha$ llb $\beta 3$ integrin engagement induces the tyrosine phosphorylation of $\mathrm{Cbl}$ and its association with phosphoinositide 3-kinase and Syk. Biochem J 2000; 351:669-76.

40. Watson SP, Auger JM, McCarty OJ, Pearce AC. GPVI and integrin $\alpha$ llb $\beta 3$ signaling in platelets. J Thromb Haemost 2005; 3:1752-62.

41. Obergfell A, Judd BA, del Pozo MA, Schwartz MA, Koretzky GA, Shattil SJ. The molecular adapter SLP-76 relays signals from platelet integrin $\alpha$ llb $\beta 3$ to the actin cytoskeleton. J Biol Chem 2001; 276:5916-23.

42. Pearce AC, McCarty OJ, Calaminus SD, Vigorito E, Turner M, Watson SP. Vav family proteins are required for optimal regulation of PLC $\gamma 2$ by integrin $\alpha$ llb $\beta 3$. Biochem J 2007; 401:753-61.

43. Ramaekers FC, Bosman FT. The cytoskeleton and disease. J Pathol 2004; 204:351-4.

44. Hotchin NA, Hall A. The assembly of integrin adhesion complexes requires both extracellular matrix and intracellular rho/rac GTPases. J Cell Biol 1995; 131:1857-65.

45. Falet H, Chang G, Brohard-Bohn B, Rendu F, Hartwig JH. Integrin $\alpha$ llb $\beta 3$ signals lead cofilin to accelerate platelet actin dynamics. Am J Physiol Cell Physiol 2005; 289:C819-25.

46. Hartwig JH, Kung S, Kovacsovics T, Janmey PA, Cantley LC, Stossel TP, Toker A. D3 phosphoinositides and outside-in integrin signaling by GPIlb/llla mediate platelet actin assembly and filopodial extension induced by phorbol 12-myristate 13-acetate. J Biol Chem 1996; 271:32986-93.

47. Fox JE, Austin CD, Reynolds CC, Steffen PK. Evidence that agonist-induced activation of calpain causes the shedding of procoagulant-containing microvesicles from the membrane of aggregating platelets. J Biol Chem 1991; 266:13289-95.

48. Nomura S, Komiyama Y, Matsuura E, Xie GL, Katsura K, Miyake T, Miyazaki Y, Kagawa H, Koike T, Fukuhara S. Participation of $\alpha$ llb $\beta 3$ in platelet microparticle generation by collagen plus thrombin. Haemostasis 1996; 26:31-7.

49. Shcherbina A, Rosen FS, Remold-O'Donnell E. Pathological events in platelets of WiskottAldrich syndrome patients. Br J Haematol 1999; 106:875-83.

50. Spronk HM, Govers-Riemslag JW, ten Cate H. The blood coagulation system as a molecular machine. Bio-essays 2003; 25:1220-8.

51. Heemskerk JW, Bevers EM, Lindhout T. Platelet activation and blood coagulation. Thromb Haemost 2002; 88:186-93.

52. Sere KM, Hackeng TM. Basic mechanisms of hemostasis. Semin Vasc Med 2003; 3:3-12.

53. Bevers EM, Comfurius P, Dekkers DW, Zwaal RF. Lipid translocation across the plasma membrane of mammalian cells. Biochim Biophys Acta 1999; 1439:317-30.

54. Butenas S, Branda RF, van't Veer C, Cawthern KM, Mann KG. Platelets and phospholipids in tissue factor-initiated thrombin generation. Thromb Haemost 2001; 86:660-7.

55. Bajaj MS, Birktoft JJ, Steer SA, Bajaj SP. Structure and biology of tissue factor pathway inhibitor. Thromb Haemost 2001; 86:959-72.

56. Hackeng TM, Sere KM, Tans G, Rosing J. Protein S stimulates inhibition of the tissue factor pathway by tissue factor pathway inhibitor. Proc Natl Acad Sci U S A 2006; 103:3106-11.

57. Sere KM, Rosing J, Hackeng TM. Inhibition of thrombin generation by protein $S$ at low procoagulant stimuli: implications for maintenance of the hemostatic balance. Blood 2004; 104:3624-30.

58. Esmon CT. Protein C anti-coagulant pathway and its role in controlling microvascular thrombosis and inflammation. Crit Care Med 2001; 29:S48-51.

59. Brecher ME. The platelet prophylactic transfusion trigger: when expectations meet reality. 
Transfusion 2007; 47:188-91.

60. Eikenboom JC, van Wordragen R, Brand A. Compliance with prophylactic platelet transfusion trigger in hematological patients. Transfus Med 2005; 15:45-8.

61. Heal JM, Liesveld JL, Phillips GL, Blumberg N. What would Karl Landsteiner do? The ABO blood group and stem cell transplantation. Bone Marrow Transplant 2005; 36:747-55.

62. Heddle NM, Cook RJ, Sigouin C, Slichter SJ, Murphy M, Rebulla P. A descriptive analysis of international transfusion practice and bleeding outcomes in patients with acute leukemia. Transfusion 2006; 46:903-11.

63. Slichter SJ. Platelet transfusion therapy. Hematol Oncol Clin North Am 1990; 4:291-311.

64. Arnold DM, Heddle NM, Kulczycky M, Carruthers J, Sigouin C, Blajchman MA. In vivo recovery and survival of apheresis and whole blood-derived platelets: a paired comparison in healthy volunteers. Transfusion 2006; 46:257-64.

65. Enright H, Davis K, Gernsheimer T, McCullough JJ, Woodson R, Slichter SJ. Factors influencing moderate to severe reactions to platelet transfusions: experience of the TRAP multicenter clinical trial. Transfusion 2003; 43:1545-52.

66. Heal JM, Blumberg N. Optimizing platelet transfusion therapy. Blood Rev 2004; 18:149-65.

67. Slichter SJ, Davis K, Enright H, Braine H, Gernsheimer T, Kao KJ, Kickler T, Lee E, McFarland J, McCullough J, Rodey G, Schiffer CA, Woodson R. Factors affecting posttransfusion platelet increments, platelet refractoriness and platelet transfusion intervals in thrombocytopenic patients. Blood 2005; 105:4106-14.

68. CBO Guideline Blood Transfusion - the Netherlands 2004.

69. Vassallo RR, Murphy S. A critical comparison of platelet preparation methods. Curr Opin Hematol 2006; 13:323-30.

70. Blajchman MA, Goldman M, Baeza F. Improving the bacteriological safety of platelet transfusions. Transfus Med Rev 2004; 18:11-24.

71. Contreras M. Diagnosis and treatment of patients refractory to platelet transfusions. Blood Rev 1998; 12:215-21.

72. Slichter SJ, Bolgiano D, Jones MK, Christoffel T, Corson J, Rose L, Foley J, Popovsky M, Baril LL, Corda T, Dincecco DM, Snyder EL. Viability and function of 8 day stored apheresis platelets. Transfusion 2006; 46:1763-9.

73. Friedmann AM, Sengul H, Lehmann H, Schwartz C, Goodman S. Do basic laboratory tests or clinical observations predict bleeding in thrombocytopenic oncology patients? A reevaluation of prophylactic platelet transfusions. Transfus Med Rev 2002; 16:34-45.

74. Janetzko K, Kluter H, van Waeg G, Eichler H. Fully automated processing of buffy coatderived pooled platelet concentrates. Transfusion 2004; 44:1052-8.

75. Gulliksson H. Platelet storage media. Transfus Apher Sci 2001; 24:241-4.

76. de Wildt-Eggen J, Nauta S, Schrijver JG, van Marwijk Kooy M, Bins M, van Prooijen HC. Reactions and platelet increments after transfusion of platelet concentrates in plasma or an additive solution: a prospective, randomized study. Transfusion 2000; 40:398-403.

77. Kerkhoffs JL, Eikenboom JC, Schipperus MS, van Wordragen-Vlaswinkel RJ, Brand R, Harvey MS, de Vries RR, Barge R, van Rhenen DJ, Brand A. A multicenter randomized study of the efficacy of transfusions with platelets stored in platelet additive solution II versus plasma. Blood 2006; 108:3210-5.

78. Keuren JF, Cauwenberghs S, Heeremans J, de Kort W, Heemskerk JW, Curvers J. Platelet ADP response deteriorates in synthetic storage media. Transfusion 2006; 46:204-12.

79. Wagner T, Vetter A, Dimovic N, Guber SE, Helmberg W, Kroll W, Lanzer G, Mayr WR, Neumuller J. Ultrastructural changes and activation differences in platelet concentrates 
stored in plasma and additive solution. Transfusion 2002; 42:719-27.

80. Hunter S, Nixon J, Murphy S. The effect of the interruption of agitation on platelet quality during storage for transfusion. Transfusion 2001; 41:809-14.

81. Callera F, DeMelo CM. Relationships between parameters used for routine quality monitoring of platelet concentrates. Transfus Med 2005; 15:161-2.

82. Slichter SJ, Harker LA. Preparation and storage of platelet concentrates. Transfusion 1976; 16:8-12.

83. Rothwell SW, Maglasang $\mathrm{P}$, Krishnamurti C. Survival of fresh human platelets in a rabbit model as traced by flow cytometry. Transfusion 1998; 38:550-6.

84. Snyder EL, Moroff G, Simon T, Heaton A. Recommended methods for conducting radiolabeled platelet survival studies. Transfusion 1986; 26:37-42.

85. Blajchman MA. The clinical benefits of the leukoreduction of blood products. J Trauma 2006; 60:S83-90.

86. Leytin V, Allen DJ, Gwozdz A, Garvey B, Freedman J. Role of platelet surface GPIb $\alpha$ and Pselectin in the clearance of transfused platelet concentrates. Transfusion 2004; 44:1487-95.

87. Furie BC, Furie B. Tissue factor pathway versus collagen pathway for in vivo platelet activation. Blood Cells Mol Dis 2006; 36:135-8.

88. Lecut C, Schoolmeester A, Kuijpers MJ, Broers JL, van Zandvoort MA, Vanhoorelbeke K Deckmyn H, Jandrot-Perrus M, Heemskerk JW. Principal role of GPVI in $\alpha 2 \beta 1$ and $\alpha$ llbb $\beta 3$ activation during collagen-induced thrombus formation. Arterioscler Thromb Vasc Biol 2004; 24:1727-33.

89. Savage B, Ginsberg MH, Ruggeri ZM. Influence of fibrillar collagen structure on the mechanisms of platelet thrombus formation under flow. Blood 1999; 94:2704-15.

90. Siljander PR, Munnix IC, Smethurst PA, Deckmyn H, Lindhout T, Ouwehand WH, Farndale RW, Heemskerk JW. Platelet receptor interplay regulates collagen-induced thrombus formation in flowing human blood. Blood 2004; 103:1333-41.

91. Kuijpers MJ, Schulte V, Bergmeier W, Lindhout T, Brakebusch C, Offermanns S, Fassler R, Heemskerk JW, Nieswandt B. Complementary roles of GPVI and $\alpha 2 \beta 1$ integrin in collageninduced thrombus formation in flowing whole blood ex vivo. Faseb J 2003; 17:685-687.

92. Nieswandt B, Watson SP. Platelet-collagen interaction: is GPVI the central receptor? Blood 2003; 102:449-61.

93. Zwaal RF, Schroit AJ. Pathophysiologic implications of membrane phospholipid asymmetry in blood cells. Blood 1997; 89:1121-32.

94. Hemker HC, Giesen PL, Ramjee M, Wagenvoord R, Beguin S. The thrombogram: monitoring thrombin generation in platelet-rich plasma. Thromb Haemost 2000; 83:589-91.

95. Kilkson $\mathrm{H}$, Holme $\mathrm{S}$, Murphy S. Platelet metabolism during storage of platelet concentrates at $22^{\circ}$ C. Blood 1984; 64:406-14.

96. Rinder HM, Snyder EL, Tracey JB, Dincecco D, Wang C, Baril L, Rinder CS, Smith BR. Reversibility of severe metabolic stress in stored platelets after in vitro plasma rescue or in vivo transfusion: restoration of secretory function and maintenance of platelet survival. Transfusion 2003; 43:1230-7.

97. Cauwenberghs S, Feijge MA, Theunissen E, Heemskerk JW, van Pampus EC, Curvers J. Novel methodology for assessment of prophylactic platelet transfusion therapy by measuring increased thrombus formation and thrombin generation. $\mathrm{Br} \mathrm{J}$ Haematol 2007; 136:480-90.

98. Metcalfe P, Williamson LM, Reutelingsperger CP, Swann I, Ouwehand WH, Goodall AH Activation during preparation of therapeutic platelets affects deterioration during storage: a comparative flow cytometric study of different production methods. $\mathrm{Br} \mathrm{J}$ Haematol 1997; 98:86-95. 
99. Boomgaard MN, Gouwerok CW, Homburg CH, de Groot G, J. IM, de Korte D. The platelet adhesion capacity to subendothelial matrix and collagen in a flow model during storage of platelet concentrates for 7 days. Thromb Haemost 1994; 72:611-6.

100. Curvers J, van Pampus EC, Feijge MA, Rombout-Sestrienkova E, Giesen PL, Heemskerk JW. Decreased responsiveness and development of activation markers of platelets stored in plasma. Transfusion 2004; 44:49-58.

101. Savion N, Varon D. Impact - the cone and plate(let) analyzer: testing platelet function and anti-platelet drug response. Pathophysiol Haemost Thromb 2006; 35:83-8.

102. Vanschoonbeek K, Feijge MA, Van Kampen RJ, Kenis H, Hemker HC, Giesen PL, Heemskerk JW. Initiating and potentiating role of platelets in tissue factor-induced thrombin generation in the presence of plasma: subject-dependent variation in thrombogram characteristics. J Thromb Haemost 2004; 2:476-84.

103. Azorsa DO, Moog S, Ravanat C, Schuhler S, Follea G, Cazenave JP, Lanza F. Measurement of GPV released by activated platelets using a sensitive immunocapture ELISA: its use to follow platelet storage in transfusion. Thromb Haemost 1999; 81:131-8.

104. Bergmeier W, Piffath CL, Cheng G, Dole VS, Zhang Y, von Andrian UH, Wagner DD. Tumor necrosis factor- $\alpha$-converting enzyme (ADAM17) mediates GPIb $\alpha$ shedding from platelets in vitro and in vivo. Circ Res 2004; 95:677-83.

105. Sano M, Williams S, Smith N, Horne M, Gralnick HR. Plasma glycocalicin in platelet concentrates: relationship to other parameters of the storage lesion. Thromb Res 1998; 92:195-8.

106. Hoffmeister KM, Josefsson EC, Isaac NA, Clausen H, Hartwig JH, Stossel TP. Glycosylation restores survival of chilled blood platelets. Science 2003; 301:1531-4.

107. Feinberg $H$, Sarin MM, Batka EA, Porter CR, Miripol JE, Stewart M. Platelet storage: changes in cytosolic $\mathrm{Ca}^{2+}$ actin polymerization and shape. Blood 1988; 72:766-9.

108. Fijnheer R, Homburg $\mathrm{CH}$, Hooibrink B, Boomgaard MN, de Korte D, Roos D. Loss of thrombin-induced $\mathrm{Ca}^{2+}$ mobilization in a subpopulation of platelets during storage. Thrombosis and Haemostasis 1991; 66:350-4.

109. Estebanell E, Diaz-Ricart M, Escolar G, Lozano M, Mazzara R, Ordinas A. Alterations in cytoskeletal organization and tyrosine phosphorylation in platelet concentrates prepared by the buffy coat method. Transfusion 2000; 40:535-42.

110. Wang C, Mody M, Herst R, Sher G, Freedman J. Flow cytometric analysis of platelet function in stored platelet concentrates. Transfus Sci 1999; 20:129-39.

111. Wadhawan V, Karim ZA, Mukhopadhyay S, Gupta R, Dikshit M, Dash D. Platelet storage under in vitro condition is associated with $\mathrm{Ca}^{2+}$-dependent apoptosis-like lesions and novel reorganization in platelet cytoskeleton. Arch Biochem Biophys 2004; 422:183-90.

112. Perrotta PL, Perrotta CL, Snyder EL. Apoptotic activity in stored human platelets. Transfusion 2003; 43:526-35.

113. Shapira S, Friedman Z, Shapiro H, Presseizen K, Radnay J, Ellis MH. The effect of storage on the expression of platelet membrane phosphatidylserine and the subsequent impact on the coagulant function of stored platelets. Transfusion 2000; 40:1257-1263.

114. Cauwenberghs S, Feijge MA, Harper AG, Sage SO, Curvers J, Heemskerk JW. Shedding of procoagulant microparticles from unstimulated platelets by integrin-mediated destabilization of actin cytoskeleton. FEBS Lett 2006; 580:5313-5320.

115. Hardy AR, Conley PB, Luo J, Benovic JL, Poole AW, Mundell SJ. P2Y $Y_{1}$ and P2Y 12 receptors for ADP desensitize by distinct kinase-dependent mechanisms. Blood 2005; 105:3552-60.

116. Cauwenberghs S, Feijge MA, Hageman G, Hoylaerts M, Akkerman JW, Curvers J, Heemskerk JW. Plasma ecto-nucleotidases prevent desensitization of purinergic receptors in stored platelets: importance for platelet activity during thrombus formation. Transfusion 2006; 
46:1018-28.

117. Brass LF, Jiang $\mathrm{H}$, Wu J, Stalker TJ, Zhu L. Contact-dependent signaling events that promote thrombus formation. Blood Cells Mol Dis 2006; 36:157-61.

118. Reid TJ, Snider R, Hartman K, Greilich PE, Carr ME, Alving BM. A method for the quantitative assessment of platelet-induced clot retraction and clot strength in fresh and stored platelets. Vox Sang 1998; 75:270-7.

119. Hoffmeister KM, Felbinger TW, Falet H, Denis CV, Bergmeier W, Mayadas TN, von Andrian UH, Wagner DD, Stossel TP, Hartwig JH. The clearance mechanism of chilled blood platelets. Cell 2003; 112:87-97.

120. Bessos H, Murphy WG, Seghatchian MJ. Monitoring the release of glycocalicin in platelet concentrates by ELISA. Blood Coagul Fibrinolysis 1991; 2:373-6.

121. Javela K, Eronen J, Sarna S, Kekomaki R. Soluble GPV as a quality marker of platelet concentrates stressed by transportation. Transfusion 2005; 45:1504-11.

122. Mazoyer E, Boizard-Boval B, Pidard D, Caen J, Wautier JL. Platelet membrane glycoproteins and platelet functions during storage in the presence of a proteinase inhibitor. Thromb Res $1991 ; 62: 165-75$

123. Rinder HM, Snyder EL. Activation of platelet concentrate during preparation and storage. Blood Cells 1992; 18:445-56; discussion 457-60.

124. Jhang JS, Spitalnik SL. Glycosylation and cold platelet storage. Curr Hematol Rep 2005; 4:483-7.

125. Bergmeier W, Burger PC, Piffath CL, Hoffmeister KM, Hartwig JH, Nieswandt B, Wagner DD Metalloproteinase inhibitors improve the recovery and hemostatic function of in vitro-aged or -injured mouse platelets. Blood 2003; 102:4229-4235.

126. Owens M, Holme S, Heaton A, Sawyer S, Cardinali S. Post-transfusion recovery of function of 5-day stored platelet concentrates. Br J Haematol 1992; 80:539-544.

127. de Korte D, Gouwerok CW, Fijnheer R, Pietersz RN, Roos D. Depletion of dense granule nucleotides during storage of human platelets. Thromb Haemost 1990; 63:275-8.

128. Prodouz KN, Moroff G. Effects of storage conditions on platelet cytoskeletal proteins. J Lab Clin Med 1985; 105:560-7.

129. Holme S, Sweeney JD, Sawyer S, Elfath MD. The expression of P-selectin during collection, processing and storage of platelet concentrates: relationship to loss of in vivo viability. Transfusion 1997; 37:12-7.

130. Scharf RE, Hanfland P. Platelet storage lesions: analysis of platelet membrane glycoproteins and platelet-derived microparticles by fluorescence-activated flow cytometry. Transfus Sci 1993; $14: 189-94$

131. Miyaji $R$, Sakai M, Urano $H$, Nakata $K$, Sakamoto $H$, Shirahata A. Decreased platelet aggregation of platelet concentrate during storage recovers in the body after transfusion. Transfusion 2004; 44:891-899.

132. Lai M, Rumi C, D'Onofrio G, Puggioni PL, Menichella G, Candido A, Leone G. Phosphatidylserine exposure in platelet concentrates during the storage period: differences between the platelets collected with different cell separators. Transfus Apher Sci 2002; 27:239-45.

133. George JN, Pickett EB, Heinz R. Platelet membrane glycoprotein changes during the preparation and storage of platelet concentrates. Transfusion 1988; 28:123-6.

134. Slichter SJ, Wandall HH, Hoffmeister KM. In vivo studies of autologous platelets stored at room temperature $\left(22^{\circ} \mathrm{C}\right), 4^{\circ} \mathrm{C}$ and $4^{\circ} \mathrm{C}$ with galactosylation. Blood (ASH Annual Meeting Abstracts) 2006; 108:580.

135. Luscher TF. Platelet-vessel wall interaction: role of nitric oxide, prostaglandins and 
endothelins. Baillieres Clin Haematol 1993; 6:609-27.

136. Rivera J, Lozano ML, Corral J, Connor J, Gonzalez-Conejero R, Ferrer F, Currie L, Vicente V. Quality assessment of platelet concentrates supplemented with second-messenger effectors. Transfusion 1999; 39:135-43.

137. Stohlawetz P, Horvath M, Pernerstorfer T, Nguyen H, Vondrovec B, Robisch A, Eichler HG Spitzauer S, Jilma B. Effects of nitric oxide on platelet activation during plateletpheresis and in vivo tracking of biotinylated platelets in humans. Transfusion 1999; 39:506-14.

138. Wong $\mathrm{K}, \mathrm{Li} \mathrm{X}$. Nitric oxide infusion alleviates cellular activation during preparation, leukofiltration and storage of platelets. Transfus Apher Sci 2004; 30:29-39.

139. Akkerman JW, Gorter G. Relation between energy production and adenine nucleotide metabolism in human blood platelets. Biochim Biophys Acta 1980; 590:107-16.

140. Verhoeven AJ, Mommersteeg ME, Akkerman JW. Balanced contribution of glycolytic and adenylate pool in supply of metabolic energy in platelets. J Biol Chem 1985; 260:2621-4.

141. Verhoeven AJ, Mommersteeg ME, Akkerman JW. Comparative studies on the energetics of platelet responses induced by different agonists. Biochem J 1986; 236:879-87.

142. Badlou BA, IJseldijk MJ, Smid WM, Akkerman JW. Prolonged platelet preservation by transient metabolic suppression. Transfusion 2005; 45:214-22.

143. Badlou BA, Spierenburg G, Ulrichts H, Deckmyn H, Smid WM, Akkerman JW. Role of GPIbo in phagocytosis of platelets by macrophages. Transfusion 2006; 46:2090-9.

144. Badlou BA, Wu YP, Smid WM, Akkerman JW. Platelet binding and phagocytosis by macrophages. Transfusion 2006; 46:1432-43.

145. Conley PB, Delaney SM. Scientific and therapeutic insights into the role of the platelet $\mathrm{P}_{2} \mathrm{Y}_{12}$ receptor in thrombosis. Curr Opin Hematol 2003; 10:333-338.

146. Gachet C. Identification, characterization and inhibition of the platelet ADP receptors. Int J Hematol 2001; 74:375-381.

147. Mahaut-Smith MP, Ennion SJ, Rolf MG, Evans RJ. ADP is not an agonist at P2X $\mathrm{X}_{1}$ receptors: evidence for separate receptors stimulated by ATP and ADP on human platelets. $\mathrm{Br} J$ Pharmacol 2000; 131:108-114.

148. Mackenzie AB, Mahaut-Smith MP, Sage SO. Activation of receptor-operated cation channels via $\mathrm{P}_{2} \mathrm{X}_{1}$ not $\mathrm{P} 2 \mathrm{~T}$ purinoceptors in human platelets. J Biol Chem 1996; 271:2879-81.

149. Sage SO, Yamoah EH, Heemskerk JWM. The roles of $P 2 X_{1}$ and $P_{2} T_{A C}$ receptors in ADPevoked $\mathrm{Ca}^{2+}$ signaling in human platelets. Cell Calcium 2000; 28:119-126.

150. Toth-Zsamboki E, Oury C, Cornelissen H, De Vos R, Vermylen J, Hoylaerts MF. P2X 1 $^{-}$ mediated ERK2 activation amplifies the collagen-induced platelet secretion by myosin light chain kinase activation. J Biol Chem 2003; 278:46661-46667.

151. Cattaneo M, Gachet C. ADP receptors and clinical bleeding disorders. Arterioscler Thromb Vasc Biol 1999; 19:2281-2285.

152. Kunapuli SP, Dorsam RT, Kim S, Quinton TM. Platelet purinergic receptors. Curr Opin Pharmacol 2003; 3:175-180.

153. Hardy AR, Jones ML, Mundell SJ, Poole AW. Reciprocal cross-talk between P2Y 1 and $P 2 Y_{12}$ receptors at the level of $\mathrm{Ca}^{2+}$ signaling in human platelets. Blood 2004; 104:1745-52.

154. Soulet C, Sauzeau V, Plantavid M, Herbert JM, Pacaud P, Payrastre B, Savi P. Gidependent and -independent mechanisms downstream of the P2 $Y_{12}$ ADP receptor. J Thromb Haemost 2004; 2:135-146.

155. Werner P, Seward EP, Buell GN, North RA. Domains of P2X receptors involved in desensitization. Proc Natl Acad Sci USA 1996; 93:5485-15490. 
156. Baurand A, Eckly A, Hechler B, Kauffenstein G, Galzi JL, Cazenave JP, Leon C, Gachet C. Differential regulation and relocalization of the platelet $P 2 Y$ receptors following activation: a way to avoid loss of hemostatic properties? Mol Pharmacol 2005; 67:721-33.

157. Marcus AJ, Broekman MJ, Drosopoulos JH, Islam N, Pinsky DJ, Sesti C, Levi R. Heterologous cell-cell interactions: thromboregulation, cerebroprotection and cardioprotection by CD39 (NTPDase-1). J Thromb Haemost 2003; 1:2497-2509.

158. Feijge MA, van Pampus EC, Lacabaratz-Porret C, Hamulyak K, Levy-Toledano S, Enouf J, Heemskerk JW. Inter-individual variability in $\mathrm{Ca}^{2+}$ signaling in platelets from healthy volunteers: effects of aspirin and relationship with expression of endomembrane $\mathrm{Ca}^{2+}$ ATPases. Br J Haematol 1998; 102:850-859.

159. Saunders AM. Sources of physiological variation in differential leukocyte counting. Blood Cells 1985:31-48.

160. Siljander P, Lassila R. Studies of adhesion-dependent platelet activation: distinct roles for different participating receptors can be dissociated by proteolysis of collagen. Arterioscler Thromb Vasc Biol 1999; 19:3033-43.

161. Schwarz UR, Geiger J, Walter U, Eigenthaler M. Flow cytometry analysis of intracellular VASP phosphorylation for the assessment of activating and inhibitory signal transduction pathways in human platelets-definition and detection of ticlopidine/clopidogrel effects. Thromb Haemost 1999; 82:1145-52.

162. Oury C, Sticker E, Cornelissen H, De Vos R, Vermylen J, Hoylaerts MF. ATP augments von Willebrand factor-dependent shear-induced platelet aggregation through $\mathrm{Ca}^{2+}$-calmodulin and myosin light chain kinase activation. J Biol Chem 2004; 279:26266-73.

163. Micheli V, Sestini S. Determining NAD synthesis in erythrocytes. Methods Enzymol 1997; 280:211-21.

164. Yang J, Wu J, Jiang H, Mortensen R, Austin S, Manning DR, Woulfe D, Brass LF. Signaling through $G_{i}$ family members in platelets. Redundancy and specificity in the regulation of adenylyl cyclase and other effectors. J Biol Chem 2002; 277:46035-46042.

165. van Giezen JJ, Humphries RG. Pre-clinical and clinical studies with selective reversible direct $P 2 Y_{12}$ antagonists. Semin Thromb Hemost 2005:195-204

166. Zimmermann $H$. Extracellular metabolism of ATP and other nucleotides. Naunyn Schmiedebergs Arch Pharmacol 2000; 362:299-309.

167. Mahaut-Smith MP, Tolhurst G, Evans RJ. Emerging roles for $P 2 X_{1}$ receptors in platelet activation. Platelets 2004; 15:131-44.

168. Hechler B, Lenain N, Marchese P, Vial C, Heim V, Freund M, Cazenave JP, Cattaneo M, Ruggeri ZM, Evans R, Gachet C. A role of the fast ATP-gated P2X ${ }_{1}$ cation channel in thrombosis of small arteries in vivo. J Exp Med 2003; 198:661-667.

169. Sevigny J, Sundberg C, Braun N, Guckelberger O, Csizmadia E, Qawi I, Imai M, Zimmermann H, Robson SC. Differential catalytic properties and vascular topography of murine nucleoside triphosphate diphosphohydrolase 1 (NTPDase1) and NTPDase2 have implications for thromboregulation. Blood 2002; 99:2801-2809.

170. Koziak K, Sevigny J, Robson SC, Siegel JB, Kaczmarek E. Analysis of CD39/ATP diphosphohydrolase (ATPDase) expression in endothelial cells, platelets and leukocytes. Thromb Haemost 1999; 82:1538-44.

171. McCullough J, Vesole DH, Benjamin RJ, Slichter SJ, Pineda A, Snyder E, Stadtmauer EA Lopez-Plaza I, Coutre S, Strauss RG, Goodnough LT, Fridey JL, Raife T, Cable R, Murphy $\mathrm{S}$, Howard Ft, Davis K, Lin JS, Metzel P, Corash L, Koutsoukos A, Lin L, Buchholz DH, Conlan MG. Therapeutic efficacy and safety of platelets treated with a photochemical process for pathogen inactivation: the SPRINT Trial. Blood 2004; 104:1534-41.

172. Lin L, Dikeman R, Molini B, Lukehart SA, Lane R, Dupuis K, Metzel P, Corash L. Photochemical treatment of platelet concentrates with amotosalen and long-wavelength 
ultraviolet light inactivates a broad spectrum of pathogenic bacteria. Transfusion 2004; 44:1496-504.

173. van der Meer PF, Pietersz RN, Reesink HW. Comparison of two platelet additive solutions. Transfus Med 2001; 11:193-7.

174. van der Meer PF, Pietersz RN, Reesink HW. Storage of platelets in additive solution for up to 12 days with maintenance of good in vitro quality. Transfusion 2004; 44:1204-11.

175. Holme S. Effect of additive solutions on platelet biochemistry. Blood Cells 1992; 18:421-30.

176. Gulliksson H, AuBuchon JP, Cardigan R, van der Meer PF, Murphy S, Prowse C, Richter E, Ringwald J, Smacchia C, Slichter S, de Wildt-Eggen J. Storage of platelets in additive solutions: a multicentre study of the in vitro effects of potassium and magnesium. Vox Sang 2003; 85:199-205.

177. Shanwell A, Falker C, Gulliksson H. Storage of platelets in additive solutions: the effects of magnesium and potassium on the release of RANTES, beta-thromboglobulin, platelet factor 4 and interleukin-7 during storage. Vox Sang 2003; 85:206-12.

178. van Rhenen DJ, Gulliksson H, Cazenave JP, Pamphilon D, Davis K, Flament J, Corash L. Therapeutic efficacy of pooled buffy-coat platelet components prepared and stored with a platelet additive solution. Transfus Med 2004; 14:289-95.

179. Brown SB, Clarke MC, Magowan L, Sanderson H, Savill J. Constitutive death of platelets leading to scavenger receptor-mediated phagocytosis. A caspase-independent cell clearance program. J Biol Chem 2000; 275:5987-96.

180. Bunescu A, Hild M, Lundahl J, Egberg N. Platelet storage in PAS-2 or autologous plasma: impact on functional parameters. Transfus Med 2001; 11:105-10.

181. de Wildt-Eggen J, Schrijver JG, Bins M, Gulliksson H. Storage of platelets in additive solutions: effects of magnesium and/or potassium. Transfusion 2002; 42:76-80.

182. Cattaneo M, Gachet C. The platelet ADP receptors. Haematologica 2001; 86:346-8.

183. Coughlin SR. Protease-activated receptors in vascular biology. Thromb Haemost 2001; 86:298-307.

184. Soslau G, Class R, Morgan DA, Foster C, Lord ST, Marchese P, Ruggeri ZM. Unique pathway of thrombin-induced platelet aggregation mediated by glycoprotein Ib. J Biol Chem 2001; 276:21173-83.

185. Keuren JF, Baruch D, Legendre P, Denis CV, Lenting PJ, Girma JP, Lindhout T. von Willebrand factor $\mathrm{C} 1 \mathrm{C} 2$ domain is involved in platelet adhesion to polymerized fibrin at high shear rate. Blood 2004; 103:1741-6.

186. Oury C, Kuijpers MJ, Toth-Zsamboki E, Bonnefoy A, Danloy S, Vreys I, Feijge MA, De Vos $\mathrm{R}$, Vermylen J, Heemskerk JW, Hoylaerts MF. Overexpression of the platelet P2X $\mathrm{X}_{1}$ ion channel in transgenic mice generates a novel pro-thrombotic phenotype. Blood 2003; 101:3969-76.

187. Cho MJ, Liu J, Pestina TI, Steward SA, Thomas DW, Coffman TM, Wang D, Jackson CW, Gartner TK. The roles of $\alpha$ llb $\beta 3$-mediated outside-in signal transduction, thromboxane $A_{2}$ and adenosine diphosphate in collagen-induced platelet aggregation. Blood 2003; 101:264651.

188. Offermanns S, Toombs CF, Hu YH, Simon Ml. Defective platelet activation in $\mathrm{G}_{\alpha q}$-deficient mice. Nature 1997; 389:183-6.

189. Weber AA, Reimann S, Schror K. Specific inhibition of ADP-induced platelet aggregation by clopidogrel in vitro. Br J Pharmacol 1999; 126:415-20.

190. Fijnheer R, Pietersz RN, de Korte D, Gouwerok CW, Dekker WJ, Reesink HW, Roos D. Platelet activation during preparation of platelet concentrates: a comparison of the plateletrich plasma and the buffy coat methods. Transfusion 1990; 30:634-8. 
191. Murphy S, Rebulla P, Bertolini F, Holme S, Moroff G, Snyder E, Stromberg R. In vitro assessment of the quality of stored platelet concentrates. The BEST (Biomedical Excellence for Safer Transfusion) Task Force of the International Society of Blood Transfusion. Transfus Med Rev 1994; 8:29-36.

192. Rinder HM, Murphy M, Mitchell JG, Stocks J, Ault KA, Hillman RS. Progressive platelet activation with storage: evidence for shortened survival of activated platelets after transfusion. Transfusion 1991; 31:409-14.

193. VandenBroeke T, Dumont LJ, Hunter S, Nixon J, Murphy S, Roger J, Herschel L, AuBuchon JP, Gulliksson H, Dengler T, Hornsey V, Prowse C. Platelet storage solution effects on the accuracy of laboratory tests for platelet function: a multi-laboratory study. Vox Sang 2004; 86:183-8.

194. Remijn JA, Wu YP, Heninga EH, IJsseldijk MJW, van Willigen G, de Groot PG, Sixma JJ, Nurden AT, Nurden P. Role of ADP receptor $P_{2} Y_{12}$ in platelet adhesion and thrombus formation in flowing blood. Arterioscler Thromb Vasc Biol 2002; 22:686-691.

195. Baurand A, Eckly A, Bari N, Léon C, Hechler B, Cazenave JP, Gachet C. Desensitization of the platelet aggregation response to ADP. Differential downregulation of the $P 2 Y_{1}$ and $P 2_{\text {cyc }}$ receptors. Thromb Haemost 2000; 84:484-491.

196. Fijnheer R, Boomgaard MN, van den Eertwegh AJ, Homburg CH, Gouwerok CW, Veldman HA, Roos D, de Korte D. Stored platelets release nucleotides as inhibitors of platelet function. Thromb Haemost 1992; 68:595-9.

197. Graff J, Klinkhardt U, Schini-Kerth VB, Harder S, Franz N, Bassus S, Kirchmaier CM. Close relationship between the platelet activation marker CD62 and the granular release of plateletderived growth factor. J Pharmacol Exp Ther 2002; 300:952-7.

198. Michelson AD, Barnard MR, Hechtman HB, MacGregor H, Connolly RJ, Loscalzo J, Valer CR. In vivo tracking of platelets: circulating degranulated platelets rapidly lose surface Pselectin but continue to circulate and function. Proc Natl Acad Sci U S A 1996; 93:11877-82.

199. de Wildt-Eggen J, Schrijver JG, Smid WM, Joie M, Bollinne V, Bins M. Platelets stored in a new-generation container differences between plasma and platelet additive solution II. Vox Sang 1998; 75:218-23.

200. Lin L, Cook DN, Wiesehahn GP, Alfonso R, Behrman B, Cimino GD, Corten L, Damonte PB, Dikeman R, Dupuis K, Fang YM, Hanson CV, Hearst JE, Lin CY, Londe HF, Metchette K, Nerio AT, Pu JT, Reames AA, Rheinschmidt M, Tessman J, Isaacs ST, Wollowitz S, Corash L. Photochemical inactivation of viruses and bacteria in platelet concentrates by use of a novel psoralen and long-wavelength ultraviolet light. Transfusion 1997; 37:423-35.

201. Ishikawa Y, Sasakawa S. Membrane potential of stored platelets and its effect on platelet functions. Thromb Res 1987; 45:265-73.

202. Mondorro TH, Shafer BC, Vostal JG. Restoration of in vitro responses in platelets stored in plasma. Am J Clin Pathol 1999; 111:693-9.

203. Verhallen PF, Bevers EM, Comfurius P, Zwaal RF. Correlation between calpain-mediated cytoskeletal degradation and expression of platelet procoagulant activity. A role for the platelet membrane-skeleton in the regulation of membrane lipid asymmetry? Biochim Biophys Acta 1987; 903:206-17.

204. Bevers EM, Comfurius P, Zwaal RF. Regulatory mechanisms in maintenance and modulation of transmembrane lipid asymmetry: pathophysiological implications. Lupus 1996; 5:480-7.

205. Fox JE. Shedding of adhesion receptors from the surface of activated platelets. Blood Coagul Fibrinolysis 1994; 5:291-304.

206. Pasquet JM, Toti F, Nurden AT, Dachary-Prigent J. Procoagulant activity and active calpain in platelet-derived microparticles. Thromb Res 1996; 82:509-22.

207. Freyssinet JM. Cellular microparticles: what are they bad or good for? J Thromb Haemost 


\section{$2003 ; 1: 1655-62$}

208. Hugel B, Martinez MC, Kunzelmann C, Freyssinet JM. Membrane microparticles: two sides of the coin. Physiol (Bethesda) 2005; 20:22-7.

209. Fox JE, Taylor RG, Taffarel M, Boyles JK, Goll DE. Evidence that activation of platelet calpain is induced as a consequence of binding of adhesive ligand to the integrin, glycoprotein Ilb-Illa. J Cell Biol 1993; 120:1501-7.

210. Ginsberg MH, Partridge A, Shattil SJ. Integrin regulation. Curr Opin Cell Biol 2005; 17:50916.

211. Schoenwaelder SM, Jackson SP, Yuan Y, Teasdale MS, Salem HH, Mitchell CA. Tyrosine kinases regulate the cytoskeletal attachment of integrin $\alpha$ llb $\beta 3$ (platelet glycoprotein Ilb/llla) and the cellular retraction of fibrin polymers. J Biol Chem 1994; 269:32479-87.

212. Lisman T, Adelmeijer J, Cauwenberghs S, Van Pampus EC, Heemskerk JW, De Groot PG. Recombinant factor VIla enhances platelet adhesion and activation under flow conditions at normal and reduced platelet count. J Thromb Haemost 2005; 3:742-51.

213. Siljander P, Farndale RW, Feijge MA, Comfurius P, Kos S, Bevers EM, Heemskerk JW. Platelet adhesion enhances the GPVI-dependent procoagulant response: involvement of p38 MAP kinase and calpain. Arterioscler Thromb Vasc Biol 2001; 21:618-627.

214. Harper AG, Sage SO. A role for the intracellular protease calpain in the activation of storeoperated calcium entry in human platelets. Cell Calcium 2007; 41:169-78.

215. Pasquet JM, Dachary-Prigent J, Nurden AT. $\mathrm{Ca}^{2+}$ influx is a determining factor of calpain activation and microparticle formation in platelets. Eur J Biochem 1996; 239:647-54.

216. Topol EJ, Byzova TV, Plow EF. Platelet GPIIb-IIla blockers. Lancet 1999; 353:227-31.

217. Calderwood DA, Shattil SJ, Ginsberg MH. Integrins and actin filaments: reciprocal regulation of cell adhesion and signaling. J Biol Chem 2000; 275:22607-10.

218. Rosado JA, Sage SO. A role for the actin cytoskeleton in the initiation and maintenance of store-mediated $\mathrm{Ca}^{2+}$ entry in human platelets. Trends Cardiovasc Med 2000; 10:327-332.

219. Shattil SJ. Integrins and Src: dynamic duo of adhesion signaling. Trends Cell Biol 2005; 15:399-403.

220. Wiedmer T, Shattil SJ, Cunningham M, Sims PJ. Role of calcium and calpain in complementinduced vesiculation of the platelet plasma membrane and in the exposure of the platelet factor Va receptor. Biochemistry 1990; 29:623-32.

221. Gemmell $\mathrm{CH}$, Sefton MV, Yeo EL. Platelet-derived microparticle formation involves GPIIb/IIla. Inhibition by RGDS and a Glanzmann's thrombasthenia defect. J Biol Chem 1993; 268:14586-9.

222. Holme PA, Solum NO, Brosstad F, Egberg N, Lindahl TL. Stimulated Glanzmann's thrombasthenia platelets produced microvesicles. Microvesiculation correlates better to exposure of procoagulant surface than to activation of GPIlb-IIla. Thromb Haemost 1995; 74:1533-40.

223. Matzdorff AC, Kuhnel G, Kemkes-Matthes B, Pralle H, Voss R, Fareed J. Effect of glycoprotein Ilb/llla inhibitors on CD62p expression, platelet aggregates, and microparticles in vitro. J Lab Clin Med 2000; 135:247-55.

224. Fox JE, Austin CD, Boyles JK, Steffen PK. Role of the membrane skeleton in preventing the shedding of procoagulant-rich microvesicles from the platelet plasma membrane. J Cell Biol 1990; 111:483-93.

225. Bennett JS. Structure and function of the platelet integrin $\alpha$ llbß3. J Clin Invest 2005; 115:3363-9.

226. Andrews RK, Gardiner EE, Shen Y, Berndt MC. Platelet interactions in thrombosis. IUBMB Life 2004; 56:13-8. 
227. Reverter JC, Beguin S, Kessels H, Kumar R, Hemker HC, Coller BS. Inhibition of plateletmediated, tissue factor-induced thrombin generation by the mouse/human chimeric 7E3 antibody. Potential implications for the effect of c7E3 Fab treatment on acute thrombosis and "clinical restenosis". J Clin Invest 1996; 98:863-74.

228. Heemskerk JWM. Calcium and platelets. In: The Molecular Basis of Calcium Action in Biology and Medicine (Pochet, R, Donato, R, Haiech, J, Heinzmann, C and Gerke, V, eds.), Kluwer Acad. Publ., The Hague (The Netherlands) 2000:45-71.

229. Offermanns S. Activation of platelet function through $\mathrm{G}$ protein-coupled receptors. Circ Res 2006; 99:1293-1304

230. Nesbitt WS, Giuliano S, Kulkarni S, Dopheide SM, Harper IS, Jackson SP. Intercellular $\mathrm{Ca}^{2+}$ communication regulates platelet aggregation and thrombus growth. J Cell Biol 2003; 160:1151-1161.

231. Woulfe D, Yang J, Prevost P, O'Brien PJ, Brass L. Signal transduction during the initiation, extension and perturbation of platelet plug formation. Platelets 2002:197-213.

232. Cauwenberghs S, van Pampus E, Curvers J, Akkerman JWN, Heemskerk JWM. Hemostatic and signaling functions of stored platelets. Transf Med Rev.; in press 2007.

233. Heemskerk JWM, Willems GM, Rook MB, Sage SO. Ragged spiking in free $\mathrm{Ca}^{2+}$ in ADPstimulated platelets: regulation of puff-like $\mathrm{Ca}^{2+}$ signal in vitro and ex vivo. J Physiol 2001; 535:625-635

234. van Gorp RM, van Dam-Mieras MC, Hornstra G, Heemskerk JW. Effect of membranepermeable sulfhydryl reagents and depletion of glutathione on $\mathrm{Ca}^{2+}$ mobilization in human platelets. Biochem Pharmacol 1997; 53:1533-42.

235. Burch PT, Burch JW. Alterations in glutathione during storage of human platelet concentrates. Transfusion 1987; 27:342-346.

236. Hervig T, Mansoor MA, Farstad M. Endogenous glutathione and platelet function in platelet concentrates stored in plasma or platelet additive solution. Transfus Med 2001; 11:97-104.

237. Merlo Pich M, Castagnoli A, Biondi A, Bernacchia A, Tazzari PL, D'Aurelio M, Parenti Castell G, Formiggini G, Conte R, Bovina C, Lenaz G. Ubiquinol and a coenzyme Q reducing system protect platelet mitochondrial function of transfusional buffy coats from oxidative stress. Free Radic Res 2002; 36:429-436.

238. Verhoeven AJ, Verhaar R, Gouwerok EG, de Korte D. The mitochondrial membrane potential in human platelets: a sensitive parameter for platelet quality. Transfusion 2005; 45:82-9.

239. Hayward CP, Rao AK, Cattaneo M. Congenital platelet disorders: overview of their mechanisms, diagnostic evaluation and treatment. Haemophilia 2006; 12:S128-136.

240. Rao AK, Jalagadugula G, Sun L. Inherited defects in platelet signaling mechanisms. Semin Thromb Hemost 2004; 30:525-535.

241. Salama ME, Raman S, Drew MJ, Abdel-Raheem M, Mahmood MN. Platelet function testing to assess effectiveness of platelet transfusion therapy. Transfus Apheresis Sci 2004; 30:93100

242. Michelson AD. Platelet function testing in cardiovascular diseases. Circulation 2004; 110:e489-93

243. Favaloro EJ. Clinical application of the PFA-100. Curr Opin Hematol 2002; 9:407-15.

244. Harrison P. The role of PFA-100 testing in the investigation and management of hemostatic defects in children and adults. Br J Haematol 2005; 130:3-10.

245. Matzdorff A. Platelet function tests and flow cytometry to monitor anti-platelet therapy. Semin Thromb Hemost 2005; 31:393-9.

246. Rinder HM. Platelet function testing by flow cytometry. Clin Lab Sci 1998; 11:365-72. 
247. Munnix IC, Strehl A, Kuijpers MJ, Auger JM, van der Meijden PE, van Zandvoort MA, oude Egbrink MG, Nieswandt B, Heemskerk JW. The GPVI-phospholipase C $\gamma 2$ signaling pathway controls thrombus formation induced by collagen and tissue factor in vitro and in vivo. Arterioscler Thromb Vasc Biol 2005; 25:2673-8.

248. van der Meijden PE, Feijge MA, Giesen PL, Huijberts M, van Raak LP, Heemskerk JW. Platelet $\mathrm{P}_{2} \mathrm{Y}_{12}$ receptors enhance signaling towards procoagulant activity and thrombin generation. A study with healthy subjects and patients at thrombotic risk. Thromb Haemost 2005; 93:1128-36.

249. Hemker HC, Giesen P, AIDieri R, Regnault V, de Smed E, Wagenvoord R, Lecompte T, Beguin S. The calibrated automated thrombogram (CAT): a universal routine test for hyperand hypocoagulability. Pathophysiol Haemost Thromb 2002; 32:249-53.

250. Hellem AJ, Borchgrevink CF, Ames SB. The role of red cells in hemostasis: the relation between hematocrit, bleeding time and platelet adhesiveness. Br J Haematol 1961; 7:42-50.

251. Valeri CR, Khuri S, Ragno G. Role of the Hct in the treatment of thrombocytopenic patients. Transfusion 2003; 43:1761-3; author reply 1763.

252. Goodrich RP, Li J, Pieters H, Crookes R, Roodt J, Heyns Adu P. Correlation of in vitro platelet quality measurements with in vivo platelet viability in human subjects. Vox Sang 2006; 90:279-85.

253. Kenet G, Lubetsky A, Shenkman B, Tamarin I, Dardik R, Rechavi G, Barzilai A, Martinowitz U, Savion N, Varon D. Cone and platelet analyser (CPA): a new test for the prediction of bleeding among thrombocytopenic patients. Br J Haematol 1998; 101:255-9.

254. Murphy S. Amphotericin B and platelet transfusion. Transfusion 1992; 32:7-8.

255. Pastakia KB, Brownson NE, Terle DA, Poindexter BJ. Amphotericin B-induced abnormalities in human platelets. Clin Mol Pathol 1996; 49:M301-M307.

256. Sloand EM, Kumar P, Yu M, Klein HG. Effect of amphotericin B and fluconazole on platelet membrane glycoproteins. Transfusion 1994; 34:415-20.

257. Matsumoto T, Wada H, Nishiyama H, Hirano T, Sakakura M, Nishii K, Masuya M, Kageyama S, Tamaki S, Nakase K, Nobori T, Shiku H. Hemostatic abnormalities and changes following bone marrow transplantation. Clin Appl Thromb Hemost 2004; 10:341-50.

258. Pihusch $M$. Bleeding complications after hematopoietic stem cell transplantation. Semin Hematol 2004; 41:93-100.

259. Cattaneo M. Platelet P2 receptors: old and new targets for anti-thrombotic drugs. Expert Rev Cardiovasc Ther 2007; 5:45-55.

260. Gachet C. Regulation of platelet functions by P2 receptors. Annu Rev Pharmacol Toxicol 2006; 46:277-300.

261. Boomgaard MN, Joustra-Dijkhuis AM, Gouwerok CW, Steneker I, Reesink HW, Loos JA, Pietersz RN, de Korte D. In vitro evaluation of platelet concentrates, prepared from pooled buffy coats, stored for 8 days after filtration. Transfusion 1994; 34:311-6.

262. Enjyoji K, Sevigny J, Lin Y, Frenette PS, Christie PD, Esch JS, Imai M, Edelberg JM, Rayburn H, Lech M, Beeler DL, Csizmadia E, Wagner DD, Robson SC, Rosenberg RD. Targeted disruption of CD39/ATP diphosphohydrolase results in disordered hemostasis and thromboregulation. Nat Med 1999; 5:1010-7.

263. Atkinson B, Dwyer K, Enjyoji K, Robson SC. Ecto-nucleotidases of the CD39/NTPDase family modulate platelet activation and thrombus formation: potential as therapeutic targets. Blood Cells Mol Dis 2006; 36:217-22.

264. Glenn JR, White AE, Johnson A, Fox SC, Behan MW, Dolan G, Heptinstall S. Leukocyte count and leukocyte ecto-nucleotidase are major determinants of the effects of adenosine triphosphate and adenosine diphosphate on platelet aggregation in human blood. Platelets $2005 ; 16: 159-70$. 
265. Heptinstall S, Johnson A, Glenn JR, White AE. Adenine nucleotide metabolism in human blood: important roles for leukocytes and erythrocytes. J Thromb Haemost 2005; 3:2331-9.

266. Ringwald J, Zimmermann R, Eckstein R. The new generation of platelet additive solution for storage at $22^{\circ} \mathrm{C}$ : development and current experience. Transfus Med Rev 2006; 20:158-64.

267. Broeders MA, Tangelder GJ, Slaaf DW, Reneman RS, oude Egbrink MG. Endogenous nitric oxide protects against thrombo-embolism in venules but not in arterioles. Arterioscler Thromb Vasc Biol 1998; 18:139-45.

268. Levi $\mathrm{M}$, ten Cate $\mathrm{H}$, van der Poll $\mathrm{T}$. Endothelium: interface between coagulation and inflammation. Crit Care Med 2002; 30:S220-4.

269. Moncada S. Adventures in vascular biology: a tale of two mediators. Philos Trans R Soc Lond 2006; 361:735-59

270. Bock M, Glaser A, Pfosser A, Schleuning M, Heim MU, Mempel W. Storage of single-donor platelet concentrates: metabolic and functional changes. Transfusion 1993; 33:311-5.

271. Kostelijk EH, Fijnheer R, Nieuwenhuis HK, Gouwerok CW, de Korte D. Soluble P-selectin as parameter for platelet activation during storage. Thromb Haemost 1996; 76:1086-9.

272. Rosado JA, Sage SO. Activation of store-mediated $\mathrm{Ca}^{2+}$ entry by secretion-like coupling between the inositol 1,4,5-trisphosphate receptor type II and human transient receptor potential (hTrp1) channels in human platelets. Biochem J 2001; 356:191-8.

273. van Gorp RM, Feijge MA, Vuist WM, Rook MB, Heemskerk JW. Irregular spiking in free $\mathrm{Ca}^{2+}$ concentration in human platelets. Regulation by modulation of the inositol trisphosphate receptors. Eur J Biochem 2002; 269:1543-1552.

274. Rosado JA, Brownlow SL, Sage SO. Endogenously expressed Trp1 is involved in storemediated $\mathrm{Ca}^{2+}$ entry by conformational coupling in human platelets. J Biol Chem 2002; 277:42157-63.

275. Hernandez-Hernandez A, Sanchez-Bernal C, Rodriguez MC, Gomez FP, Llanillo M, Sanchez-Yague J. Loss of phosphotyrosine phosphatase activity and changes in the tyrosine phosphorylation state of proteins after storage of sheep platelets in plasma or Seto solution at $4^{\circ} \mathrm{C}$. Vox Sang $2001 ; 81: 241-7$.

276. Pasquet JM, Dachary-Prigent J, Nurden AT. Comparison between the loss of platelet membrane asymmetry, microvesiculation and the tyrosine phosphorylation of proteins Prostaglandins Leukot Essent Fatty Acids 1997; 57:451-3.

277. Sommeijer DW, Joop K, Leyte A, Reitsma PH, ten Cate H. Pravastatin reduces fibrinogen receptor GPIIla on platelet-derived microparticles in patients with type 2 diabetes. J Thromb Haemost 2005; 3:1168-71.

278. Keuren JF, Magdeleyns EJ, Govers-Riemslag JW, Lindhout T, Curvers J. Effects of storageinduced platelet microparticles on the initiation and propagation phase of blood coagulation. $\mathrm{Br} J$ Haematol 2006; 134:307-13.

279. Bevers EM, Wiedmer T, Comfurius P, Shattil SJ, Weiss HJ, Zwaal RF, Sims PJ. Defective $\mathrm{Ca}^{2+}$-induced microvesiculation and deficient expression of procoagulant activity in erythrocytes from a patient with a bleeding disorder: a study of the red blood cells of Scott syndrome. Blood 1992; 79:380-8.

280. Cauwenberghs S, Feijge MAH, Harper AG, Sage SO, van Pampus E, Curvers J, Heemskerk JWM. Shedding of procoagulant microparticles from platelets through integrin outside-in signaling involving WASP and the actin cytoskeleton. Blood (ASH Annual Meeting Abstracts) 2006; 108:1529.

281. DeMali KA, Wennerberg K, Burridge K. Integrin signaling to the actin cytoskeleton. Curr Opin Cell Biol 2003; 15:572-82.

282. Bode AP, Orton SM, Frye MJ, Udis BJ. Vesiculation of platelets during in vitro aging. Blood $1991 ; 77: 887-95$ 
283. Mason KD, Carpinelli MR, Fletcher JI, Collinge JE, Hilton AA, Ellis S, Kelly PN, Ekert PG, Metcalf D, Roberts AW, Huang DCS, Kile BT. Programmed anuclear cell death delimits platelet life span. Cell 2007; 128:1173.

284. Li J, Xia Y, Bertino AM, Coburn JP, Kuter DJ. The mechanism of apoptosis in human platelets during storage. Transfusion 2000; 40:1320-9.

285. van Engeland M, Ramaekers FCS, Schutte B, Reutelingsperger CPM. A novel assay to measure loss of plasma membrane asymmetry during apoptosis of adherent cell in culture. Cytometry 1996; 24:131-139.

286. Flaumenhaft R. Formation and fate of platelet microparticles. Blood Cells Mol Dis 2006; 36:182-7.

287. Babic AM, Josefsson EC, Bergmeier W, Wagner DD, Kaufman RM, Silberstein LE, Stossel TP, Hartwig JH, Hoffmeister KM. In vitro function and phagocytosis of galactosylated platelet concentrates after long-term refrigeration. Transfusion 2007; 47:442-51.

288. Cognasse F, Boussoulade F, Chavarin P, Acquart S, Fabrigli P, Lamy B, Garraud O. Release of potential immunomodulatory factors during platelet storage. Transfusion 2006; 46:1184-9

289. Bergmeier W, Rabie T, Strehl A, Piffath CL, Prostredna M, Wagner DD, Nieswandt B. GPVI downregulation in murine platelets through metalloproteinase-dependent shedding. Thromb Haemost 2004; 91:951-8.

290. Ringwald J, Althoff F, Zimmermann R, Strasser E, Weisbach V, Zingsem J, Eckstein R. Washing platelets with new additive solutions: aspects on the in vitro quality after 48 hours of storage. Transfusion 2006; 46:236-43.

291. Sweeney J, Kouttab N, Holme S, Kurtis J, Cheves T, Nelson E. Storage of platelet-rich plasma-derived platelet concentrate pools in plasma and additive solution. Transfusion 2006; 46:835-40.

292. Carcao MD, Blanchette VS, Stephens D, He L, Wakefield CD, Butchart S, Christie DJ, Rand $\mathrm{ML}$. Assessment of thrombocytopenic disorders using the Platelet Function Analyzer (PFA100). Br J Haematol 2002; 117:961-4.

293. Michelson AD, Frelinger AL, 3rd, Furman MI. Current options in platelet function testing. Am J Cardiol 2006; 98:4N-10N

294. Brand A, Novotny V, Tomson B. Platelet transfusion therapy: from 1973 to 2005. Hum Immunol 2006; 67:413-8.

295. Veihola M, Aroviita P, Linna M, Sintonen H, Kekomaki R. International comparison of the technical efficiency of component preparation. Transfusion 2006; 46:2109-14.

296. Veihola M, Aroviita P, Linna M, Sintonen H, Kekomaki R. Variation of platelet production and discard rates in 17 blood centers representing 10 European countries from 2000 to 2002. Transfusion 2006; 46:991-5.

297. Standards for blood banks and transfusion services. 23rd ed. Bethesda: American Association of Blood Banks 2004

298. Cardigan R, Sutherland J, Wadhwa M, Dilger $P$, Thorpe R. The influence of platelet additive solutions on cytokine levels and complement activation in platelet concentrates during storage. Vox Sang 2003; 84:28-35.

299. van der Meer PF, Gulliksson H, Aubuchon JP, Prowse C, Richter E, de Wildt-Eggen J. Interruption of agitation of platelet concentrates: effects on in vitro parameters. Vox Sang 2005; 88:227-34

300. Blajchman MA. Substitutes and alternatives to platelet transfusions in thrombocytopenic patients. J Thromb Haemost 2003; 1:1637-41.

301. Piccin A, Murphy WG, Smith OP. Circulating microparticles: pathophysiology and clinical 
implications. Blood Rev 2006.

302. Mause SF, von Hundelshausen P, Zernecke A, Koenen RR, Weber C. Platelet microparticles: a transcellular delivery system for RANTES promoting monocyte recruitment on endothelium. Arterioscler Thromb Vasc Biol 2005; 25:1512-8.

303. Norol F, Bierling P, Roudot-Thoraval F, Ferrer Le Coeur F, Rieux C, Lavaux A, Kuentz M, Duedari N. Platelet transfusion: a dose-response study. Blood 1998; 92:1448-1453. 


\section{Dankwoord}


Het maken van een proefschrift doe je niet op je eentje. Daarom wil ik graag de volgende mensen bedanken voor hun hulp de afgelopen vier jaar.

Prof. Dr. J. Rosing, promotor. Graag wil ik u bedanken omdat u mij de mogelijkheid heeft gegeven mijn promotie-onderzoek binnen het Cardiovasculair Onderzoeks Instituut Maastricht (CARIM) te verrichten. Toen ik eind 2002 mijn open solliciatie aan u richtte, heeft u mij doorverwezen naar Johan Heemskerk en zo ben ik binnen CARIM op het project van de 'bloedplaatjes voor transfusie doeleinden' terecht gekomen. Op deze manier heb ik eveneens de kans gekregen om aan verschillende cursussen deel te nemen en mijn kennis op het vakgebied van zowel de Vasculaire Biologie als de Cardiologie uit te breiden.

Dr. J.W.M. Heemskerk, co-promotor. Beste Johan, de eerste keer dat we elkaar 'in levende lijve' ontmoetten, was begin 2003 in het West-Vlaamse Kortrijk waar ik stage had gelopen bij Dr. H. Deckmyn. Vervolgens kreeg ik in februari 2003 een plekje binnen jouw groep. In het begin hadden we het af en toe moeilijk om elkaar te verstaan, letterlijk en figuurlijk. Ik weet dat dat voor een groot stuk lag aan mijn eigen koppigheid, maar deels ook aan een Belgisch/Nederlandse taal- en culturele barrière waarbij er op een andere manier 'tussen de regels' wordt gelezen. Zonder jou stond ik met mijn promotieonderzoek niet waar ik nu sta. Dank, voor die vele uren teksten verbeteren, al dat gepuzzel met stofjes en signaalwegen en zo veel meer waar ik geen weet van heb.

Dr. J. Curvers, co-promotor. Beste Joyce, ik vond het fijn om met jou te mogen samenwerken. Af en toe viel het promoveren niet mee, maar na een 'babbel' met jou kon ik er weer tegenaan. Op de verschillende NVB meetings, ISTH congressen en uitstapjes naar het CLB in Amsterdam hebben we elkaar beter leren kennen en is er een vertrouwensband ontstaan. Ik vind het een spijtige zaak dat het plaatjesonderzoek bij Sanquin in Maastricht ophoudt met bestaan, maar zoals ik al eerder zei 'is dit misschien wel het einde van een samenwerking, maar zeker niet het einde van een vriendschap'. Ik wens je nog 
een fijne tijd toe als klinisch chemicus 'in spé' en als moeder van twee mooie kindjes.

Dr. E. van Pampus, hemato-oncoloog in het AZM. Beste Lizzy, tijdens mijn promotie-onderzoek was jij de link naar de praktijk. Dankzij jou heb ik geleerd waarom het belangrijk is om wetenschappelijk onderzoek in functie van de patiënt te zien. Dank voor de vele discussies betreffende de fysiologie van plaatjestransfusies. Het is wonderbaarlijk om te zien hoe jij met zoveel liefde en toewijding je beroep uitoefent!

Mijn directe collega's binnen de plaatjesgroep bij Biochemie. Beste Marion, wat hebben we toch ons hoofd zitten breken over die micropartikels! Uiteindelijk is het toch allemaal goed gekomen na een tripje naar Engeland. Ons uitstapje naar het Queens College in Cambridge bij Dr. S. Sage was een ervaring die ik nooit zal vergeten. Dank voor de vele gesprekjes over zowel 'het promotieonderzoek' als 'de dingen des levens'. Het heeft me heel vaak geholpen de 'dingen' weer in zijn perspectief te plaatsen. Ik hoop dat we in de toekomst nog vaak met de stafkaart de heuvels van Limburg zullen verkennen. Gezien onze gezamenlijke achtergrond in scouting, twijfel ik daar geen seconde aan! Wie weet, krijgen we Simone nog wel vaker mee ... . Imke, onze tripje door Zuidoost-Azië was één van de mooiste en avontuurlijkste reizen die ik ooit gemaakt heb. Snorkelen in het heldere water in Zuid-Thailand, inktvis eten uit de 'steamboat', met de lokale bus op zoek naar de drijvende markt in Bangkok, ... heerlijk was dat toch! Dat (bloem)kaarsje bij 'Big Buddha' heeft toch geholpen, want ons boekje is eindelijk af! Dank voor de vele 'babbels' en uitstapjes in Maastricht. Marijke, alhoewel ik de laatste tijd iets minder mee ben gaan sporten (waar zou dat nu toch aan liggen?), is het mede dankzij jou dat mijn conditie op pijl is gebleven. Onze autorit naar Würzburg met de muizen op de achterbank, weliswaar veilig achter de gordel, staat in mijn geheugen gegrift! Was me dat een ritje zeg; met een 'skodake' tussen al die 'vlammende' Duitse BMW's. Beste Judith en Paola, voor jullie zit het er ook bijna op! Ik wens jullie veel succes met zowel het afronden van jullie proefschrift als de toekomst die daarop volgt. Nog een bedankje voor de stimulerende discussies tijdens de werkbesprekingen, waardoor ik steeds weer even verder moest doordenken 
over de proefjes en de resultaten. Saskia en Karen. Voor jullie is het avontuur nog maar pas begonnen. Ik wens jullie eveneens veel succes in de toekomst. Geniet ook van de extraatjes die erbij horen!

Andere (oud)collega's binnen de vakgroep Biochemie. Ha Kim, nu is het jouw beurt. Het was aan het begin van ons promotie-onderzoek, bij het 'kopieermachien' aan de toenmalige 'ping-pong' tafel, dat we tegen elkaar zeiden 'jou ken ik ergens van'. Een maandje eerder waren we elkaar namelijk tegengekomen op een sollicitatie-gesprek in Leuven. Er heerste een behoorlijke spanning tussen de kandidaten en wij waren de enige twee die daar vrolijk met elkaar zaten te kletsen. In de tussentijd hebben we festivals afgeschuimd, vooral veel 'kriekskes' achterover gegoten en Australië doorkruist. Ons laatste avontuur in Amerika, waar we de lancering van een space shuttle hebben gezien, vrolijk aan tafel zijn gaan zitten bij VIPs in de hemostase wereld en vooral in de verkeerde richting de snelweg zijn opgereden, zal ik nooit of te nimmer vergeten. Een vriendschap waartegen ik zeg 'to be continued ...' . Beste Simone, je was tijdens die vier jaar een luisterend oor waar ik steeds bij terecht kon, tijdens de lunch en ook daarnaast. Zo hebben we wel vaker stiekem onze lunchtijd met een half uurtje laten uitlopen, he. Nog veel plezier bij het toneelspelen en tot op de volgende wandeltocht in het Limburgse heuvelland? Beste Theo, scherpe vragen met af en toe een vleugje humor - dat kan ik wel appreciëren. Edouard, dank voor de stimulerende discussies betreffende boedplaatjes en stolling. Lisbeth, hopelijk mag ik volgend jaar nog een keertje van die de lekkere smoutebollen van uw mama komen smullen? Als ik het goed heb, komt binnenkort 'Pirates of the Caribbean' nummer 3 uit. Tot dan. Betta, de manier waarop jij niet alleen met wetenschappelijke vraagstukken maar evenzeer met mensen omgaat, is wonderbaarlijk. Voor mij ben je iemand met een groot hart en vooral een behoorlijke hoeveelheid incasseringsvermogen. Stella, ik herinner me nog die keer dat je een poging hebt gedaan om mij te leren scaten aan het kanaal in Kanne, met een veegborstel zodat ik mijn evenwicht niet zou verliezen. Het spijt me ten zeerste - ik bak er nog steeds niets van. Tot bij het heffen van het volgende glaasje spiritus! Kenneth, Svetlana, Sarah, Arne, Machiel, Rachel, Hugo, Kristof, 
Connie, Marina en Julian (en al de andere AIO's die er in tussentijd zijn bijgekomen), veel succes met jullie proefschrift. Cees, Leon, Kirsten en MarieJosé, Paul, Mieke, Guido, Tilman, Rory, Kristin en Wenke, Gerry, Hugo, Henri en José, Chris, Nico, Lisette, Petra, Cecile, en Heidi, Leo, Menno, Tosca, Ketie en Yvette, bedankt dat ik altijd bij jullie deur aan mocht kloppen. Tenslotte, wil ik jou bedanken, Trees, voor de lekkere koffie die me er elke ochtend weer doorsleepte en voor het regelen van praktische zaken, vooral op het einde van dit promotie-onderzoek.

Mijn collega's bij Sanquin. Beste Eva, tijdens deze laatste maanden is het me pas duidelijk geworden hoe sterk jij je als manager hebt ingezet en bent opgekomen voor de plaatjesgroep in Maastricht. Heel veel dank hiervoor! Judith, ik heb je de laatste maanden eigenlijk pas goed leren kennen. Je bent een tolerant iemand met een open en positieve geest. Dank voor al het 'geregel' van leuke en minder leuke dingen. Ellen, eveneens dank voor het regelen van praktische zaken. Nog effkes doorbijten en dan breekt er voor jou ook weer een nieuwe periode aan. Karin, het einde van jouw promotieonderzoek, over de motivatie van mensen om bloed te geven, komt er ook bijna aan. Veel succes met het afronden en de mogelijke post-doc baan hierna. Jeffrey, ik kende jou al van toen je nog AIO was bij de universiteit. Het was fijn om samen met jou bij de bloedbank aan het plaatjesonderzoek te werken. Inmiddels gaat jouw carrière een andere kant uit. Veel succes in de toekomst als klinisch chemicus. Elke, het is altijd plezierig met jou erbij! Tot op het volgende (oud)bloedbank uitje! Aan al die andere mensen bij de bloedbank in Maastricht, Nijmegen en Amsterdam, dank voor de fijne samenwerking en de stimulerende discussies. Aan diegenen binnen Sanquin, die mij de kans hebben gegeven om 'die ene lezing' te geven op de BEST meeting in Amsterdam. Dank voor deze unieke ervaring!

De leden van de beoordelingscommissie. Prof. Dr. F.C.S. Ramaekers (voorzitter), Prof. Dr. Ph.G. de Groot (Universiteit Utrecht), Prof. Dr. A. Brand (Sanquin Bloedbank - Universiteit Leiden), Prof. Dr. H. ten Cate en Dr. M.G.A. oude Egbrink. Hartelijk dank voor het kritisch doornemen en beoordelen van dit proefschrift. 
External collaborations. Prof. Dr. J.W.N. Akkerman, Prof. Dr. M. Hoylaerts, Prof. Dr. B. Nieswandt, Prof. Dr. H. Deckmyn, Dr. C. Oury, Dr. G. Hageman and Dr. S.O. Sage. Thank you for all the help with experiments and for the stimulating discussions, that gave the articles an extra dimension.

Aan al die mensen die vrijwillig bloed kwamen geven voor ons onderzoek. Duizend maal dank! Zonder jullie zouden mijn aders er niet meer zo fraai uitzien!

Vrienden, van ver en dichtbij. Brunhilde, Hans en Katrien. Ik hoop dat we over tien jaar nog steeds naar elkaar toe trekken om gezellig met elkaar (bij) te kletsen. Cecile en Jos, Veerle, Els, Veke, Patricia, Sabine en andere oudkotgenoten. We hebben met elkaar 'lief en leed' gedeeld, wat ertoe heeft geleid dat we nu reeds 10 jaar lang er elk jaar voor een weekendje met z'n allen op uittrekken. Mijn oude scouts-vrienden. Het is elke keer weer fijn om terug bij elkaar te zijn (waar dat dan ook mag zijn)!

Familie. Mama en papa. Dankzij jullie zin voor avontuur, heb ik vanaf jonge leeftijd al heel wat van de wereld kunnen zien. Dank voor alle steun de afgelopen jaren. Het was niet altijd gemakkelijk, vooral die eerste jaren in Leuven. Zonder jullie had ik op dit moment niet gestaan waar ik nu sta! Een dikke knuffel ook aan mijn meter, mijn tweede mama. Lidia, ik herinner me nog goed hoe ik zo'n twintig jaar geleden bij jou op de kamer sliep in het bovenste stapelbed. Terwijl ik 's avonds naar een cassetje van de Bereboot luisterde vlak voor het inslapen, zat jij je huiswerk te maken. Nu zijn het jouw kindjes die bij mij op de kamer slapen! Ik geniet er telkens weer van wanneer jullie in België zijn. Nancy, een tweede grote zus om naar op te kijken; al hebben we in onze puber- en tienerjaren wel vaker aan elkaars haren zitten trekken. Nu ben ik meter van jouw dochtertje en daar geniet ik van, dat weet je. Het is fijn om nog steeds jullie kleine zusje te zijn! Dikke kus, Sa'ke. 


\section{Curriculum vitae}


Sandra Cauwenberghs was born on the $16^{\text {th }}$ of April 1979 in Anderlecht, Belgium. While living in Vilvoorde, she completed high school at the Maria Assumpta Lyceum te Laken (Brussels) in 1997. The same year, she started the study of Pharmacy at the University of Leuven, and in 2000 she obtained her bachelor degree. This was followed by a master study in Biochemistry at the same university. She made her graduation internship at the Laboratory for Thrombosis Research (LATRON, IRC-KULAK, Belgium) under the supervision of Dr. H. Deckmyn. In July 2002, she finished her master degree in Biochemistry and graduated with distinction. On February $1^{\text {st }} 2003$, she started as a PhD student at the Sanquin Blood Centre Maastricht and the Department of Biochemistry, Cardiovascular Research Institute Maastricht (CARIM), University of Maastricht, the Netherlands. The research performed there on the activation state and excitability of transfusion platelets is supervised by Dr. J.W.M. Heemskerk (CARIM) and Dr. J. Curvers (Bloodbank). After taking several courses in the field of hemostasis and thrombosis and cardiovascular research, she received the CARIM PhD training certificate in 2006. During this period, she visited several conferences on hemostasis and thrombosis and on transfusion science in Oxford and Birmingham (UK), Utrecht and Amsterdam (NL), Antwerp (Belgium), Sydney (Australia) and Orlando (USA). During her $\mathrm{PhD}$ period she visited the platelet laboratories of Dr. H. Deckmyn (Kortrijk, Belgium), Dr. M. Hoylaerts (Leuven, Belgium), Dr. S. Sage (Cambridge, UK) and Dr. B. Nieswandt (Wurzburg, Germany) and collaborated with the laboratory of Dr. Ph. de Groot (Utrecht, the Netherlands) and Dr. Pochet (Brussels, Belgium). In February 2007, she started as a post-doctoral researcher at the Sanquin Blood Bank (Maastricht, the Netherlands). 


\section{Publications}

\section{Papers}

1. Ulrichts $\mathrm{H}$, Vanhoorelbeke $\mathrm{K}$, Cauwenberghs $\mathbf{S}$, Vauterin $\mathrm{S}$, Kroll H, Santoso S, Deckmyn $H$. "Von Willebrand factor, but not $\alpha$-thrombin binding to platelet glycoprotein $\mathrm{lb} \alpha$ is influenced by the HPA-2 polymorphism." Arteriosclererosis Thrombosis and Vascular Biology (2003). 23:1302-7.

2. Lisman T, Adelmeijer J, Cauwenberghs S, Van Pampus EC, Heemskerk JW, de Groot PG. "Recombinant factor VIla enhances platelet adhesion and activation under flow conditions at normal and reduced platelet count." J Thromb Haemost (2005). 3:742-51.

3. Keuren J, Cauwenberghs S, Heeremans J, de Kort W, Heemskerk JW, Curvers J. "Platelet ADP response deteriorates in synthetic storage media." Transfusion (2006). 46:204-12.

4. Cauwenberghs S, Feijge MA, Hageman G, Hoylaerts M, Akkerman JW, Curvers $\mathrm{J}$, Heemskerk JW. "Plasma ecto-nucleotidases prevent desensitization of purinergic receptors in stored platelets: importance for platelet activity and thrombus formation." Transfusion (2006). 46:1018-28.

5. Daubie V, Cauwenberghs $\mathbf{S}$, Senden NH, Pochet R, Lindhout T, Buurman WA, Heemskerk JW. "Factor $\mathrm{Xa}$ and thrombin evoke additive calcium and proinflammatory responses in endothelial cells subjected to coagulation." Biochimica et Biophysica Acta (2006). 1763:860-9.

6. Cauwenberghs S, Feijge MA, Harper AG, Sage SO, Curvers J, Heemskerk JW. "Shedding of procoagulant microparticles from unstimulated platelets by integrinmediated destabilization of actin cytoskeleton." FEBS Letters (2006). 580:5313-20.

7. Cauwenberghs S, Feijge MA, Theunissen E, Heemskerk JW, Van Pampus EC, Curvers J. "Novel methodology for assessment of prophylactic platelet transfusion therapy by measuring increased thrombus formation and thrombin generation." British Journal of Hematology (2007). 136:480-90.

8. Cauwenberghs S, van Pampus EC, Curvers J, Akkerman JW, Heemskerk JW. "Hemostatic and signaling functions of transfused platelets." Transfusion Medicine Reviews (in press).

9. Cauwenberghs S, Curvers J, Feijge MA, Heemskerk JW. "Effect of storage on agonist-induced calcium responses in platelets: a pilot study." (to be submitted). 


\section{Abstracts}

1. Ulrichts $\mathrm{H}$, Vanhoorelbeke $\mathrm{K}$, Cauwenberghs S, Vauterin S, Kroll B, Santoso $\mathrm{S}$, Deckmyn H. "The platelet glycoprotein Ib $\alpha$ HPA-2 polymorphism affects VWF binding, but not thrombin interaction." Journal of Thrombosis and Hemostasis (2003). July, suppl. 1 - OC173.

2. Lisman T, Adelmeijer J, Cauwenberghs S, van Pampus EC, Heemskerk JW, de Groot PG. "Recombinant factor VIla enhances platelet adhesion and aggregation under flow conditions at normal and reduced platelet count." Blood (2004). 104 P2618.

3. Cauwenberghs S, Feijge MA, Curvers J, Heemskerk JW "Preservation of platelet $\mathrm{P}_{2} \mathrm{X}_{1}$ and P2Y receptor function in plasma." Platelets (2004). 15: 355-393.

4. Cauwenberghs S, Feijge MA, Hoylaerts M, Hageman G, van Pampus EC, Akkerman JW, Curvers J, Heemskerk JW. "Plasma ecto-NTPDase prevents platelet $\mathrm{P}_{2} \mathrm{X}_{1}$ and $\mathrm{P}_{2} \mathrm{Y}_{12}$ receptor desensitization during storage." Journal of Thrombosis and Hemostasis (2005) 3, suppl. 1 - P0987.

5. Cauwenberghs S, Feijge MA, Keuren JF, van Pampus EC, Curvers J, Heemskerk JW. "Integrins mediate shedding of procoagulant microparticles at minimal platelet activation." Journal of Thrombosis and Hemostasis (2005). 3, suppl. 1 - P1584.

6. Curvers J, Cauwenberghs S, Heeremans J, de Kort W, Heemskerk JW, Keuren JF. "Platelet ADP receptor function rapidly deteriorates in additive solutions." Journal of Thrombosis and Hemostasis (2005). 3 suppl. 1 - P2179.

7. Cauwenberghs S, Feijge MA, Keuren JF, van Pampus EC, Curvers J, Heemskerk JW. "Integrins mediate shedding of procoagulant microparticles via the actin cytoskeleton at minimal platelet activation." Platelets (2006). 17: 108-125.

8. Cauwenberghs S, Feijge MA, Heemskerk JW, van Pampus EC, Curvers J. Assessment of the clinical benefit of prophylactic platelet transfusion in thrombocytopenia using novel methodology to measure thrombus formation and thrombin generation. American Society of Hematology (ASH) (2006), Blood (in press).

9. Cauwenberghs S, Feijge MAH, Harper AG, Sage SO, van Pampus ECM, Curvers $\mathrm{J}$, Heemskerk JWM. Shedding of procoagulant microparticles from platelets through integrin outside-in signaling involving WASP and the actin cytoskeleton. American Society of Hematology (ASH) (2006), Blood (in press). 


\section{Oral presentations}

1. Plasma ecto-nucleotidases prevent desensitization of purinergic receptor function on stored platelets. European Platelet Meeting (2005) - Utrecht, the Netherlands.

2. Plasma ecto-nucleotidases prevent desensitization of purinergic receptor function on stored platelets. Dutch Society of Blood Transfusion (NVBT) (2005) - Utrecht, the Netherlands.

3. Plasma ecto-nucleotidases prevent desensitization of purinergic receptors in stored platelets. Belgian Society of Thrombosis and Hemostasis (BSTH) (2005) Antwerp, Belgium.

4. Platelet function in thrombocytopenic blood : effect of platelet transfusion. Dutch Society of Blood Transfusion (NVBT) (2006) - Utrecht, the Netherlands.

5. Platelet function in thrombocytopenic blood : effect of platelet transfusion. Central Laboratory for Blood Transfusion (CLB) (2006) - Amsterdam, the Netherlands.

6. Hemostatic properties of platelets stored for transfusion purposes. Central Laboratory for Blood Transfusion (CLB) (2006) - Amsterdam, the Netherlands.

7. Hemostatic properties of platelets stored for transfusion purposes. Cardiovascular Research Institute Maastricht (CARIM - Dept. of Biochemistry) (2006) Maastricht, the Netherlands.

8. Signaling and hemostatic function of transfused platelets. Sanquin Wetenschapsdag (2006) - Amsterdam, the Netherlands.

9. Shedding of procoagulant microparticles from platelets through integrin outside-in signaling involving (N)-WASP and the actin cytoskeleton. American Society of Hematology (ASH) (2006) - Orlando, USA.

10. Assessment of platelet function in thrombocytopenic blood: effect of platelet transfusion. Invited speaker on first Scott Murphy Memorial Lecture of The Biomedical Excellence for Safer Transfusion (BEST) Collaborative meeting (2007) - Amsterdam, the Netherlands. 
